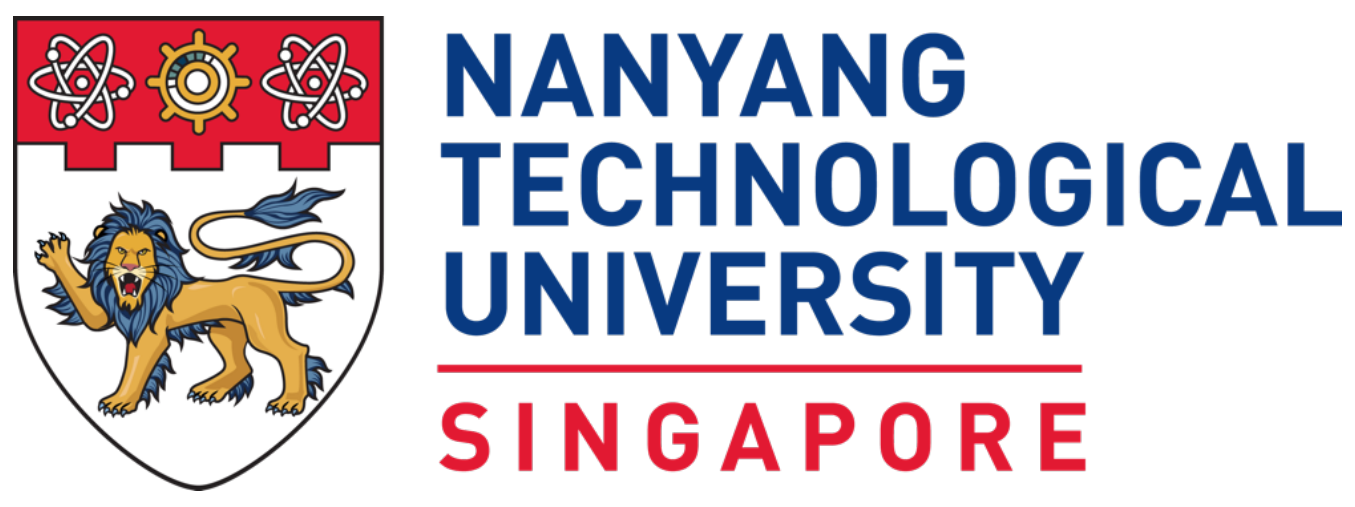

BIOPHYSICAL CHARACTERIZATION OF NATURAL ANTIMICROBIALS TOWARDS DEVELOPMENT OF ANTIBACTERIAL SKIN THERAPIES

\author{
ELBA RUTH VALLE GONZÁLEZ
}

SCHOOL OF MATERIALS SCIENCE AND ENGINEERING 



\title{
BIOPHYSICAL CHARACTERIZATION OF NATURAL ANTIMICROBIALS TOWARDS DEVELOPMENT OF ANTIBACTERIAL SKIN THERAPIES
}

\author{
ELBA RUTH VALLE GONZÁLEZ
}

SCHOOL OF MATERIALS SCIENCE AND ENGINEERING

A thesis submitted to the Nanyang Technological University in partial fulfilment of the requirement for the degree of Doctor of Philosophy 



\section{Statement of Originality}

I hereby certify that the work embodied in this thesis is the result of original research, is free of plagiarised materials, and has not been submitted for a higher degree to any other University or Institution.

$23 \mathrm{Jul} 2020$

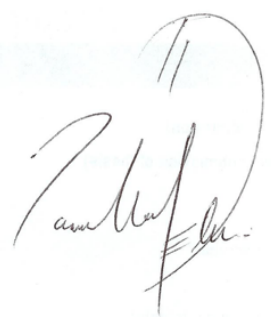

Date

Elba Ruth Valle González 



\section{Supervisor Declaration Statement}

I have reviewed the content and presentation style of this thesis and declare it is free of plagiarism and of sufficient grammatical clarity to be examined. To the best of my knowledge, the research and writing are those of the candidate except as acknowledged in the Author Attribution Statement. I confirm that the investigations were conducted in accord with the ethics policies and integrity standards of Nanyang Technological University and that the research data are presented honestly and without prejudice.

24 Jul 2020

Date

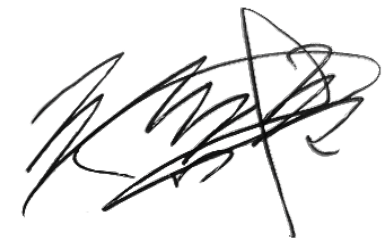

MSE Full Professor Nam-Joon Cho 



\section{Authorship Attribution Statement}

Chapter 4 is published substantially as Valle-González, E. R.; Jackman, J. A.; Yoon, B. K.; Park, S.; Sut, T. N.; Cho, N.-J., Characterizing How Acidic pH Conditions Affect the Membrane-Disruptive Activities of Lauric Acid and Glycerol Monolaurate. Langmuir 2018, 34 (45), 13745-13753. DOI: 10.1021/acs.langmuir.8b02536. Reproduced with permission, Copyright (2018) American Chemical Society.

The contributions of the co-authors are as follows:

Professor Nam-Joon Cho provided the initial project direction and edited the manuscript drafts

I wrote the manuscript drafts and feedback was received from Dr. Joshua A. Jackman and Professor Cho

I prepared all samples, conducted fluorescence spectroscopy experiments, bacterial growth evaluation, and antibacterial assays, including experimental design, execution, and data analysis

Mr. Tun Naw Sut assisted with quartz crystal microbalance-dissipation experiments, and I designed and interpreted the experimental results

Ms. Soohyun Park assisted with fluorescence microscopy experiments and data organization, and I designed and interpreted the experimental results

Chapter 5 is published substantially as Valle-González, E. R., Jackman, J. A., Yoon, B. K., Mokrzecka, N. \& Cho, N.-J. pH Dependent Antibacterial Activity of Glycolic Acid: Implications for Anti-Acne Formulations. Scientific Reports 10, 7491 (2020). DOI: 10.1038/s41598-020-64545-9. Reproduced with permission, Copyright (2020) Springer Nature.

The contributions of the co-authors are as follows:

Professor Nam-Joon Cho provided the initial project direction and edited the manuscript drafts 
I prepared the manuscript drafts. The manuscript was revised by Dr Jackman and Professor Cho

I designed the study, performed material synthesis, antibacterial assays, confocal microscopy, bioluminescence testing, and analyzed all experimental data

Dr. Bo Kyeong Yoon assisted in the editing of the final manuscript

Ms. Natalia Mokrzecka aided in the performance of the antibacterial assays and confocal microscopy

Chapter 6 was partially published as Sut, T. N; Valle-González, E. R.; Yoon, Bo Kyeong; Park, Soohyun; Jackman, Joshua A.; Cho, Nam-Joon. Bicelle Engineering for MembraneDisruptive Antibacterial Applications. Applied Materials Today (2021). Reproduced with permission, Copyright (2021) .

The contributions of the co-authors are as follows:

Professor Cho suggested the materials area and edited the manuscript drafts

I wrote the drafts of the manuscript. The manuscript was revised together with Dr. Jackman and Professor Cho

I co-designed the study with Dr. Bo Kyeong Yoon and she provided feedback of the final manuscript editing

I executed the material synthesis, DLS characterization, antibacterial assays, confocal microscopy and data analysis

Mr. Tun Naw Sut assisted with QCM-D experiments, and I designed and interpreted the experimental results

Ms. Soohyun Park assisted with fluorescence microscopy experiments and I designed and interpreted the experimental results

All the Cryo-TEM, including sample preparation, was conducted by the staff in the Electron Microscopy facilities at the NTU Institute of Structural Biology

23 Jul 2020

Date

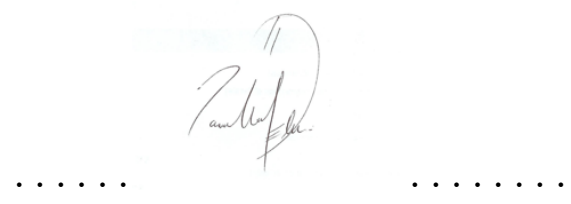

Elba Ruth Valle González 


\section{Abstract}

Acne vulgaris and atopic dermatitis are the world's most common skin diseases. Both are associated with the overgrowth of Cutibacterium acnes bacteria (formerly known as Propionibacterium acnes) for the first one and Staphylococcus aureus for the second. While direct inhibition of $C$. acnes and S. aureus can improve therapeutic outcomes, this goal is difficult to achieve due to the growing rise of antibiotic-resistant strains and current therapies rely mainly on ameliorating disease symptoms. Improved treatment strategies are needed to directly inhibit $C$. acnes and $S$. aureus, and mitigate disease symptoms. One promising bioinspired option involves utilizing natural antimicrobial lipids and glycolic acid. Antimicrobial lipids are found in the epidermis and are designed to protect against undesired bacteria and regulate the microbiome. Glycolic acid is a well-known compound in skincare used to diminish wrinkles and marks. To further improve the cutaneous delivery of the formulation with antimicrobial lipids, bicelles were devised. Bicelles are a new type of nanocarriers that have proved to be effective in dermal uptakes by guaranteeing the local effect, skin appendage penetration and time controlled delivery. The overall hypothesis of this thesis was that the therapeutic activities of the antimicrobial lipids assembled as bicelles and glycolic acid can be employed in additive multifunctional combination to treat C. acnes and $S$. aureus infections. To test the hypothesis, an integrated combination of chemical, physical and biological experimental approaches were undertaken. First the antibacterial effect of antimicrobial lipids, namely lauric acid and glycerol monolaurate, was characterized by employing model membrane platforms and establishing correlations with antibacterial activity. Secondly, the anti-infective activity of glycolic acid against $C$. acnes was evaluated. The mechanism by which glycolic acid destroys the cell membrane was determined using a fluorescent counterstaining technique to distinguish live and dead cells, and by monitoring a sensitive marker for membrane damage. Third, bicelles were developed for the protection, delivery and effect enhancement of the antimicrobial lipid on skin. The bicelles size was resolved with light scattering and electron microscopy to match it with the antimicrobial activity. Finally, the cell proliferation and viability were tested over human immortalized keratinocyte cells $(\mathrm{HaCaT})$ to prove its safeness. Taken together these outcomes, it was proven that glycerol monolaurate and glycolic acid are a viable and 
feasible solution to treat $C$. acnes and $S$. aureus infections. It provide mechanistic insight into how antimicrobial lipids, glycolic acid and bicelles with glycerol monolaurate operate and destroy bacterial membranes under skin-relevant environmental conditions. 


\section{Lay Summary}

Acne vulgaris and atopic dermatitis are the world's most common skin diseases. Acne vulgaris is caused by the excessive growth of Cutibacterium acnes bacteria and atopic dermatitis is aggravated by Staphylococcus aureus overpopulation. The conventional treatment for both involves the use of antibiotics and other drugs to improve the symptoms. However, antibiotics no longer work because bacteria keep mutating and developing resistance. This is a serious public health problem and new solutions are urgently needed to defeat bacteria. In this sense, antimicrobial lipids and glycolic acid are a promising solution. Antimicrobial lipids are naturally found in skin and products like coconut oil, and their function is to protect against undesirable bacteria. On the other hand, glycolic acid is a well-known compound in skincare used to reduce wrinkles and marks. To further ensure the penetration of the antimicrobial lipids and glycolic into the skin a delivery vehicle of nano size was utilized. Bicelles are an innovative vehicle capable to deliver compounds in the deeper skin layers and protect them from the environment, allowing for a better effect. The objective of this thesis was to develop a new therapy to treat acne and atopic dermatitis infections with antimicrobial lipids (lauric acid or glycerol monolaurate) fabricated as bicelles in combination with glycolic acid. By making connection between biological, chemical and physical principles, it was explained how antimicrobial lipids, glycolic acid and the bicelles kill bacteria. The novelty of this research is the possibility to design an effective, yet mild treatment, which eliminates the undesirable bacteria and provides relief for the symptoms, avoiding antibiotic resistance and side effects. Together all these findings may contribute to the development of new drugs, decrease the patients expenses by reducing the number of purchased products and visits to the specialist, and most importantly, improve the quality of life of millions of people suffering from these diseases. 


\section{Acknowledgements}

Before everything else, I want to thank God for making my dream come true, allowing me to study at one of the most prestigious universities in the world. For uncountable blessings, and for making me understand his plans are perfect (Jeremiah, 29:11).

This dissertation would not have been possible without the scholarship and funding provided from NTU and SINGA. I want to express my sincere gratitude for conferring me a once in a life time opportunity to do my doctoral studies and continue developing my passion for skincare science.

I am deeply grateful to Prof. Nam-Joon Cho, my supervisor, for believing in me and giving me the opportunity to work in his lab. To Prof. Maurice Van Steensel, my co-supervisor, for taking me under his wing and trusting me on the development of some of his unique work. I am most grateful to Prof. Joshua A. Jackman for his guidance and mentorship all these four years.

A very special gratitude goes out to all the NTU staff and lab mates, particularly to Dr. Bo Kyeong Yoon, for teaching me all the basis for this research.

To my tireless cheerleader, my mom, who supported me spiritually and emotionally throughout this journey. Thank you for your endless encouragement and love, and for being the inspiration and refuge I always needed. To my dad, grandma, brother and friends, thanks for always being there.

To Steffen Battran, my partner, for his love demonstrations, patience and motivation. For being the best life mate I could ever ask for.

Lastly, to my Singaporean family and the incentive to be a successful woman: Sasha Tinwalla, Wipula Paquet, Sigalit Meker, Oded Halevi, Gaia Ferracci and Teng-Fei Fan. ¡Muchas gracias! 


\section{Table of Contents}

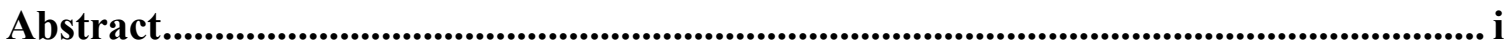

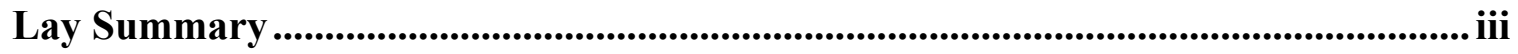

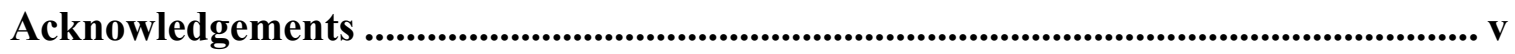

Table of Contents ............................................................................................... vii

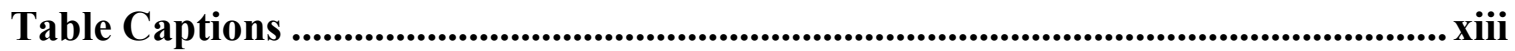

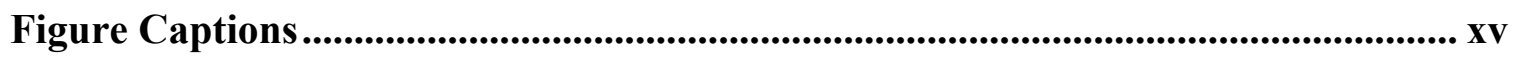

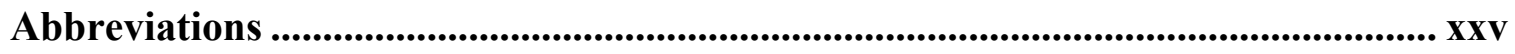

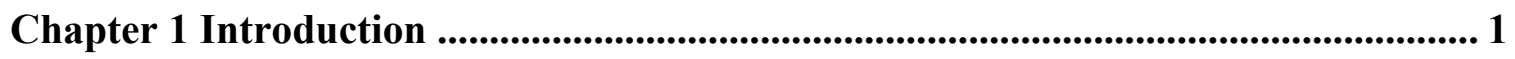

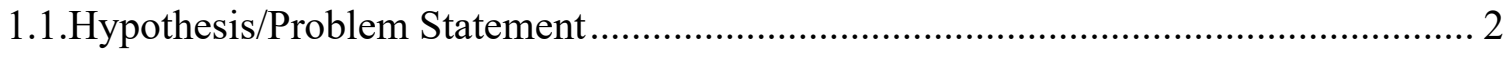

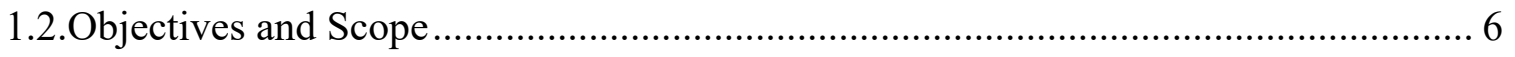

1.3.Dissertation Overview ..................................................................................... 8

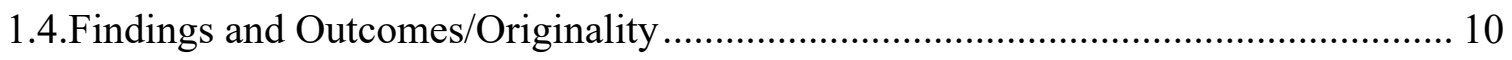

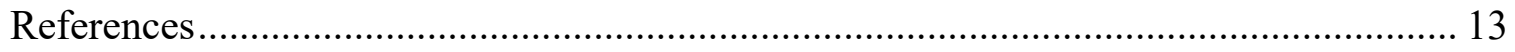

Chapter 2 Literature Review ............................................................................ 19

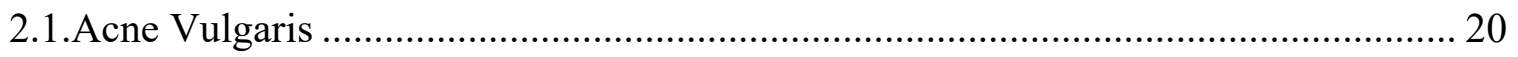

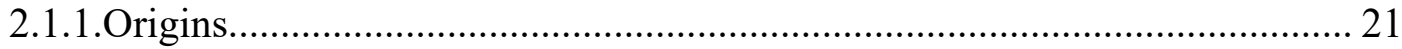

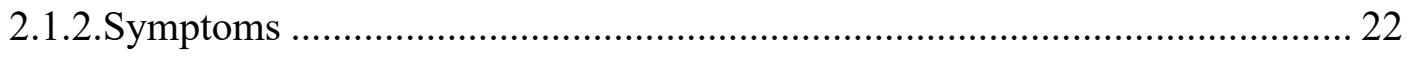

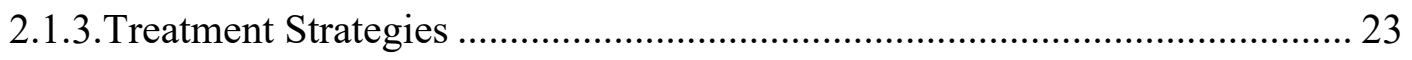

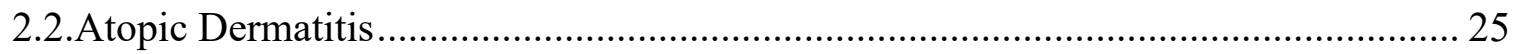

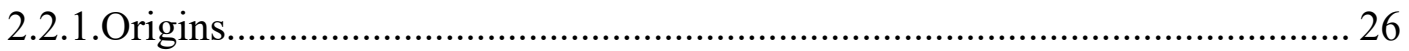

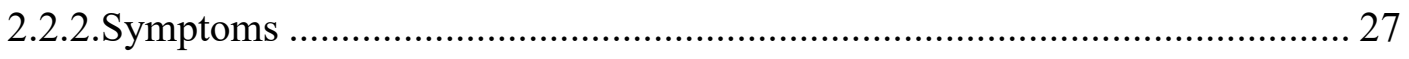

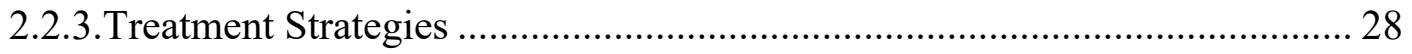

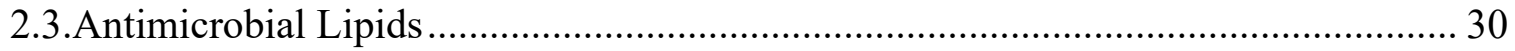




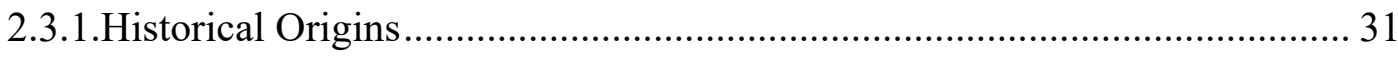

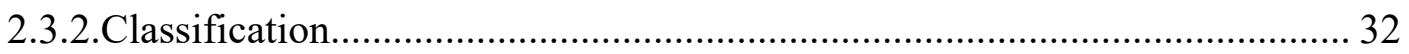

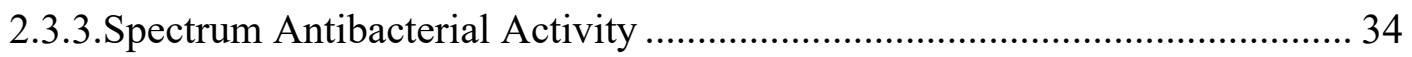

2.3.4.Mechanism of Antimicrobial Activity ................................................... 35

2.3.5.Experimental Characterization of Antimicrobial Lipids............................. 36

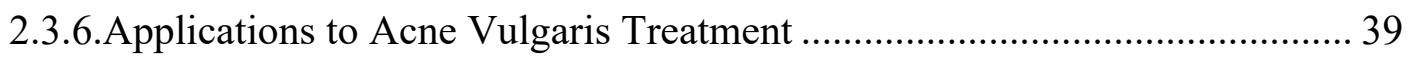

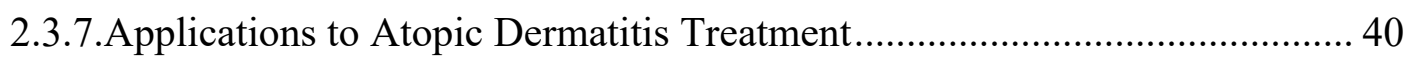

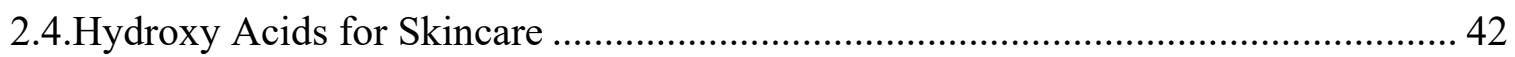

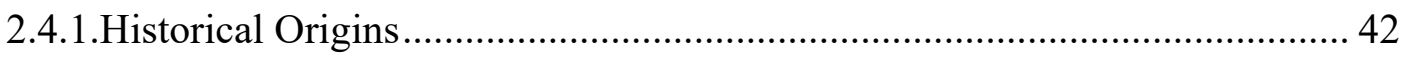

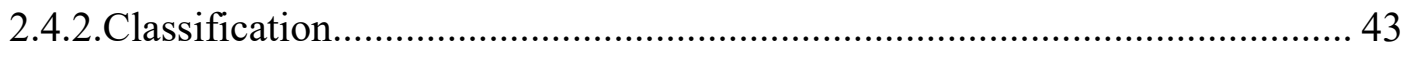

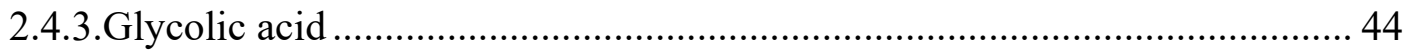

2.4.4. Experimental Characterization of Glycolic acid .................................... 46

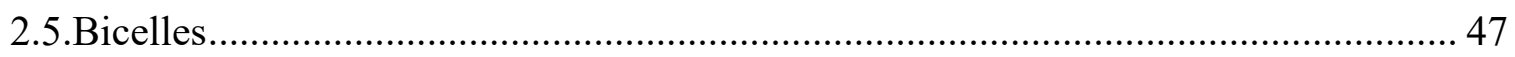

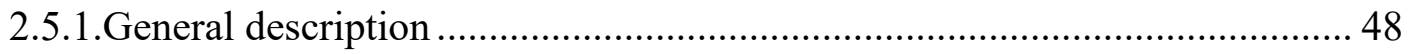

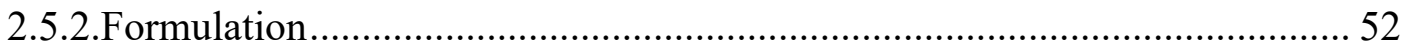

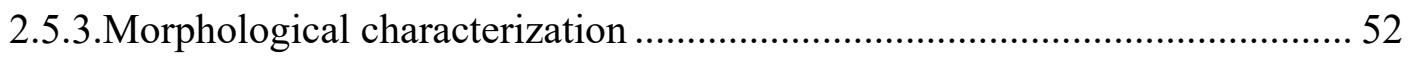

2.5.4.Application of Bicelles to the Skin ...................................................... 55

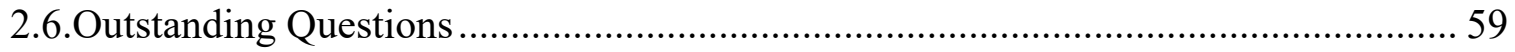

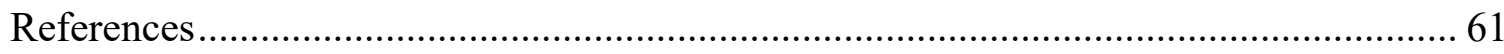

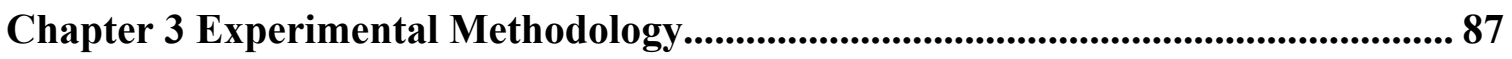

3.1.Rationale for the Selection of Materials and Experimental Techniques.................... 88

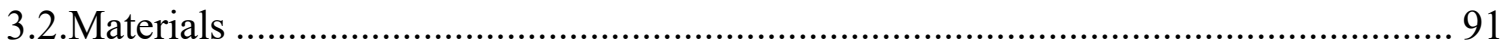

3.2.1.Single Chain Lipid Amphiphiles .......................................................... 91

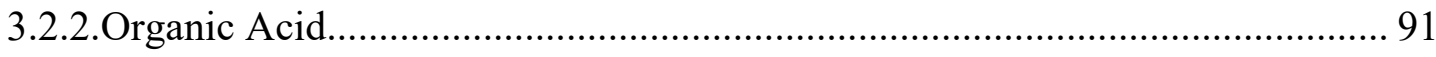

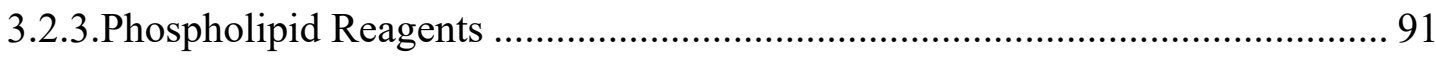

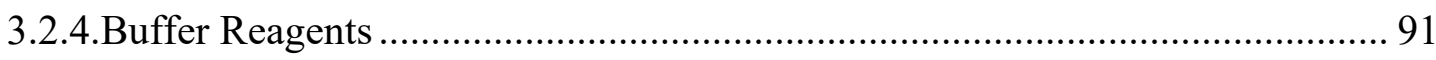

3.2.5.Bacterial Cell Strains and Antibacterial Testing Reagents............................ 92

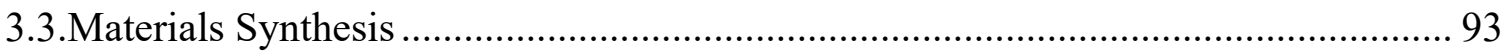

3.3.1.Preparation of Antimicrobial Lipid Solutions........................................ 93 


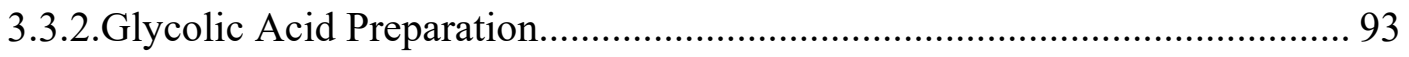

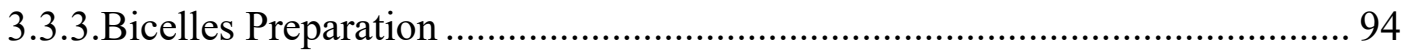

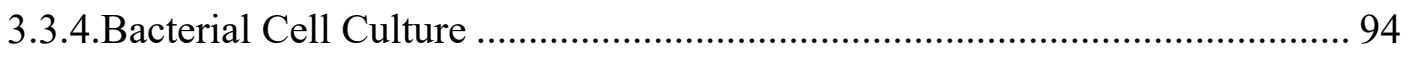

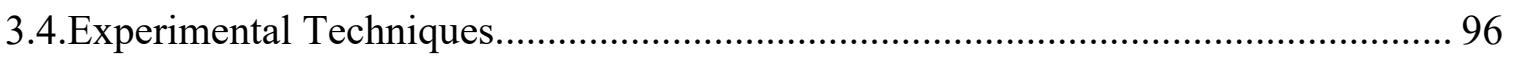

3.4.1.Critical Micelle Concentration Determination Assay................................. 96

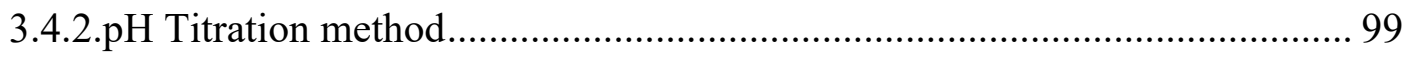

3.4.3.Quartz Crystal Microbalance-Dissipation.............................................. 101

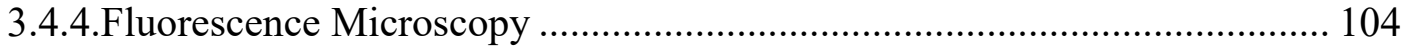

3.4.5.Minimum Inhibitory Concentration (MIC) Testing................................. 106

3.4.6.Minimum Bactericidal Concentration (MBC) Testing ............................. 109

3.4.7.Live/Dead Bacterial Cell Staining ............................................................. 110

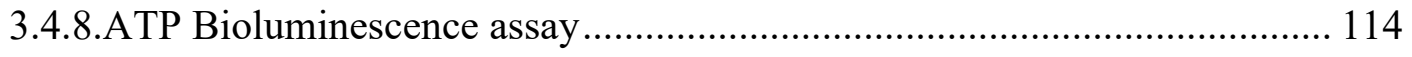

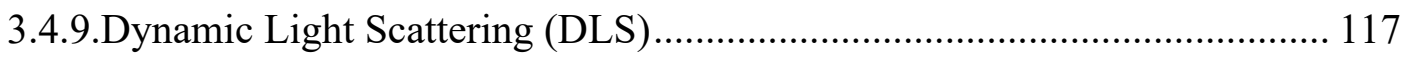

3.4.10.Cryo-Transmission Electron Microscopy (TEM) .................................... 119

3.4.11.Cell Viability Testing................................................................ 121

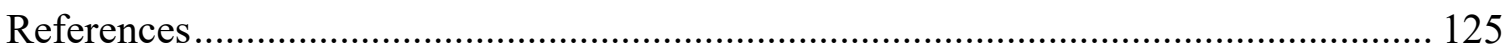

Chapter 4 Characterizing How Acidic pH Conditions Affect the MembraneDisruptive Activities of Lauric Acid and Glycerol Monolaurate

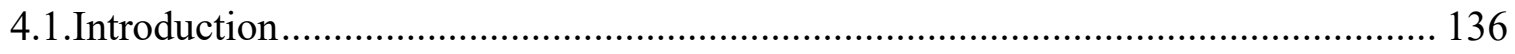

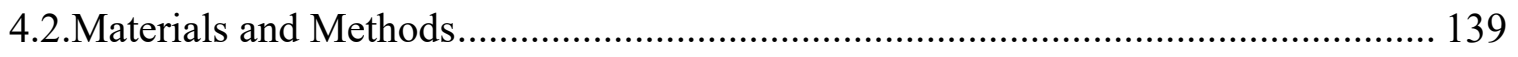

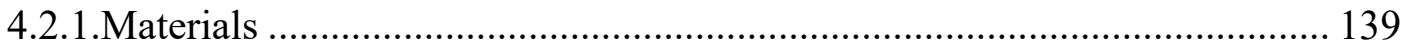

4.2.2.Preparation of Antimicrobial Lipid Solutions........................................ 139

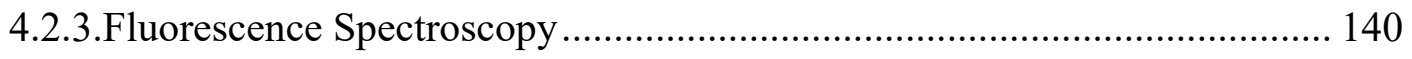

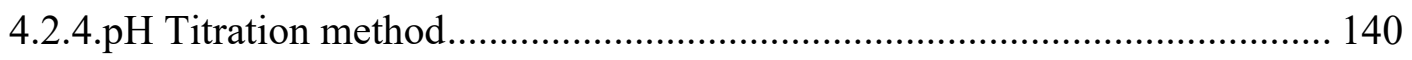

4.2.5.Quartz Crystal Microbalance-Dissipation............................................. 140

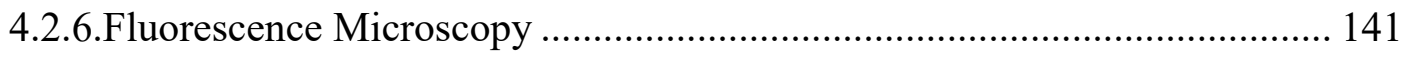

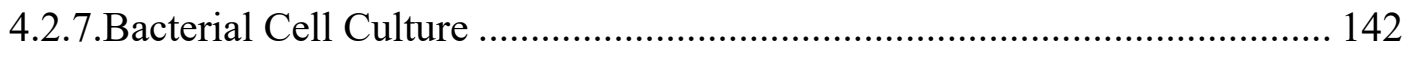

4.2.8.Minimum Inhibitory Concentration (MIC) Assay .................................. 143

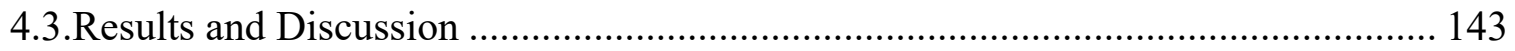




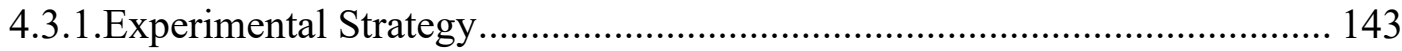

4.3.2.Effect of Solution $\mathrm{pH}$ on Critical Micelle Concentration ............................. 144

4.3.3.Determination of the $\mathrm{pK}_{\mathrm{a}}$ value of Lauric acid with the $\mathrm{pH}$ Titration method .. 146

4.3.4.Effect of Compound Treatment on Supported Lipid Bilayers...................... 148

4.3.5. Observation of Membrane Morphological Responses................................... 154

4.3.6. Evaluation of Antibacterial Activity .......................................................... 162

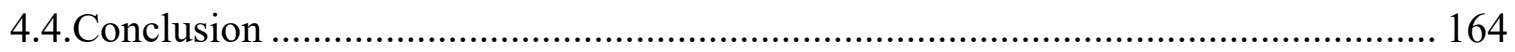

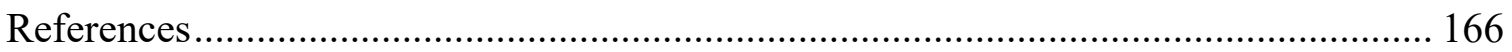

Chapter 5 pH-Dependent Antibacterial Activity of Glycolic Acid: Implications for Anti-Acne Formulations

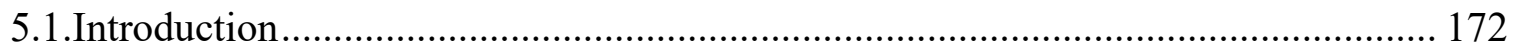

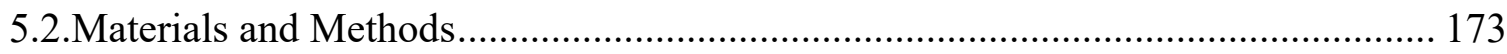

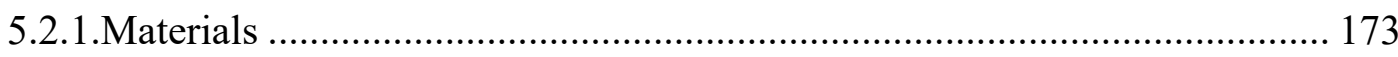

5.2.2.Glycolic Acid Preparation........................................................................... 174

5.2.3.Bacterial Cell Culture ........................................................................... 174

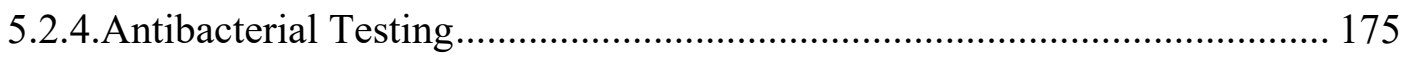

5.2.5.Minimum Bactericidal Concentration (MBC) Testing ................................ 175

5.2.6.Live/Dead Bacterial Cell Staining ............................................................. 176

5.2.7.ATP Release Measurements .................................................................. 176

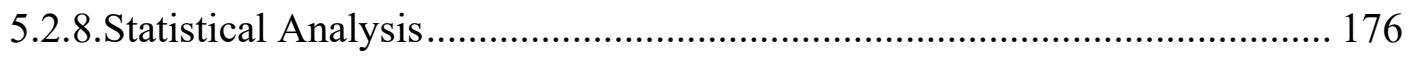

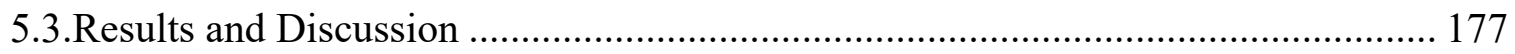

5.3.1.Antibacterial potency as a function of solution $\mathrm{pH}$.................................... 177

5.3.2. Characterization of bactericidal activity ..................................................... 179

5.3.3.Microscopic observation of bacterial cell killing.......................................... 182

5.3.4.Evaluation of membrane permeabilization ................................................... 184

5.3.5.Implications for anti-acne formulation development................................... 185

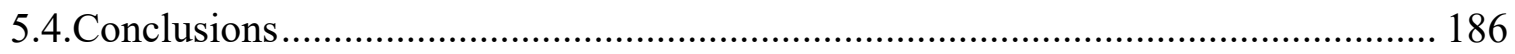


References 187

Chapter 6 Engineering Phospholipid-Glycerol Monolaurate Bicellar Systems with Membrane-Disruptive Activity against Synthetic and Bacterial Cell Membranes

6.1.Introduction 192

6.2.Materials and Methods. 195

6.2.1.Reagents . 195

6.2.2.Bicelle Preparation. 195

6.2.3.Quartz Crystal Microbalance-Dissipation (QCM-D)................................ 196

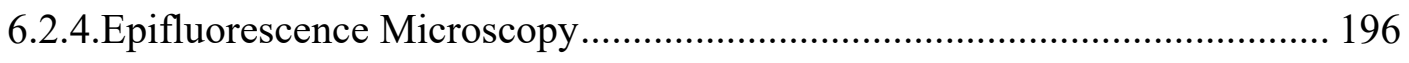

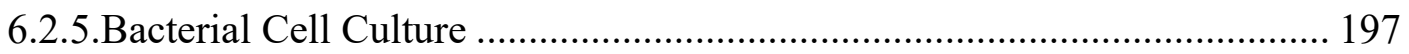

6.2.6.Cryogenic Transmission Electron Microscopy (Cryo-TEM) ..................... 197

6.2.7.Minimum Bactericidal Concentration (MBC) Testing .............................. 197

6.2.8.Live/Dead Bacterial Cell Staining ............................................................ 198

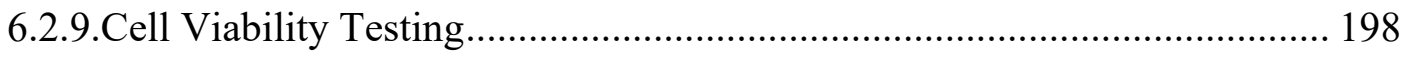

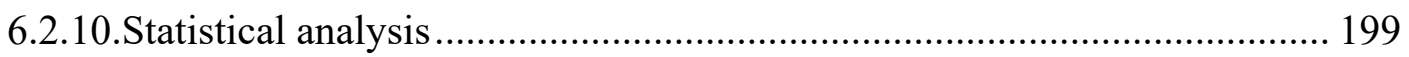

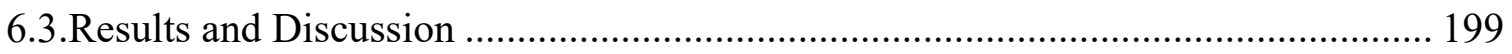

6.3.1.Evaluation of Bicelle Adsorption and SLB Formation.............................. 199

6.3.2.Evaluation of Membrane-Disruptive Activity of Bicelles ......................... 205

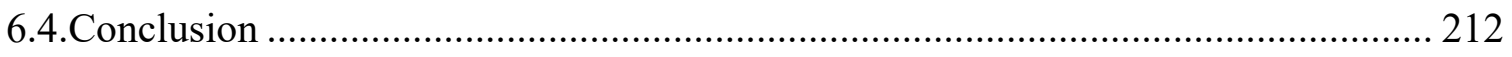

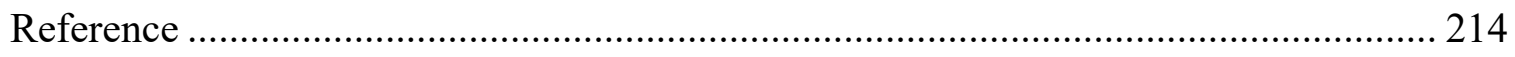

Chapter 7 Conclusion and Future Work ............................................................. 221

7.1.Discussion and Concluding Remarks ........................................................... 222

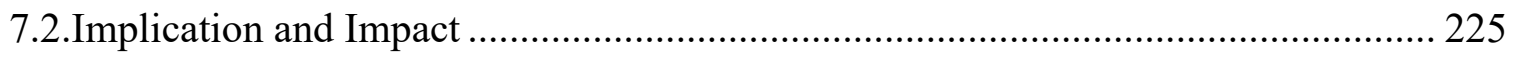

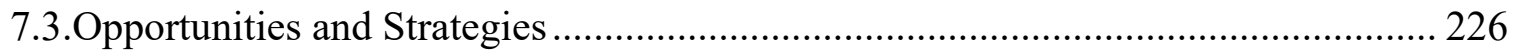

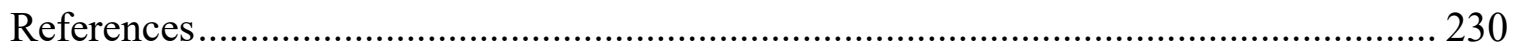




\section{Table Captions}

Table 2.1. Global evaluation of acne disease status.

Table 2.2. Contributing steps in disease pathogenesis, and currently available treatments.

Table 2.3. Prescribed treatments for mild to moderate atopic dermatitis.

Table 2.4. Medium-chain fatty acids and monoglycerides.

Table 2.5. Alpha hydroxy acids for skincare.

Table 2.6 Lipid composition of bicelles and encapsulated ingredients for skin applications 


\section{Figure Captions}

Figure 2.1. Contributors to acne development.

Figure 2.2. Molecular structure of Glycolic acid.

Figure 2.3. Routes of delivery systems across the epidermis. The possible pathways are: (a) through the appendages, (b) through the corneocytes (transcellular route) and (c) through the matrix layer (intercellular route).

Figure 2.4. Schematic representation of the ideal bicelle model. The flat bilayer region is formed by long-chain phospholipids, normally DMPC, and the rim of short-chain phospholipids, DHPC.

Figure 2.5. Different nanostructures adopted by bicellar systems. The morphology of the bicellar system depends on the type, concentration and ratio of long/short -chain phospholipids. Long-chain lipids, in dark grey, tend to form bilayers and assemble in flatter areas. Short-chain lipids, in light grey, are micelle prone and reorganize in curved areas. (A) spherical micelles, (B) discoidal micelles (bicelles), (C) tubular structures, (D) branched structures and (E) perforated lamellar sheets.

Figure 3.1 Chemical structure of 1-pyrenecarboxaldehyde. Fluorophore used to determine the CMC values in this thesis.

Figure 3.2. Graph of the equivalence point and $\mathrm{pK}_{\mathrm{a}}$ value of lauric acid in water. $\mathrm{A}$ titration curve was plotted by measuring the $\mathrm{pH}$ as a function of the volume of $\mathrm{NaOH}$. The equivalence point, center of the vertical region of the curve, was determined to be 7 (red dashed line) and the $\mathrm{pK}_{\mathrm{a}}$ value, half of the titration volume of $\mathrm{NaOH}$, was 5.8.

Figure 3.3. Diagram of the oscillation decay in QCM-D to measure the shift in dissipation. (A) The voltage is applied across the electrodes. (B) The oscillatory decay and 
pattern of a viscoelastic film when the quartz crystal disc comes to rest after switching off the voltage. Image modified from Biolin Scientific/Q-Sense.

Figure 3.4. Schematic diagram of the main components of an epi-fluorescence microscope.

Figure 3.5. Schematic representation of a plate tested for MIC and MBC after incubation. After incubation under anaerobic or aerobic conditions the samples were spotted onto agar plates and reincubated for the final assessment of bacterial occurrence. The minimum inhibitory concentration (MIC) was determined as the lowest amount of antibacterial agent that exerted a bacteriostatic behavior. The minimum bactericidal concentration was defined as the lowest amount of antibacterial agent killing $99.99 \%$ of bacteria. All experiments were accompanied by their Positive (bacteria in buffer) and Negative (buffer alone) controls to minimize the effect of other variables.

Figure 3.6. Example of images taken with CLSM using the Live/Dead BacLight Bacterial Viability Kit with $C$. acnes bacteria. Live bacteria cells are dyed in green (Left panel) while dead in red (right panel). The positive control is $C$. acnes in PBS and the negative in ethanol $90 \%$. Bacterial concentration of $1 \times 10^{8} \mathrm{CFU} \mathrm{mL}^{-1}$. Scale bar of 100 $\mu \mathrm{m}$.

Figure 3.7. Schematic representation of the confocal laser scanning microscope (CLSM) operation principle. The adjustable iris, pinhole, only allows light from the confocal plane of the specimen to reach the photomultiplier. Image retrieved from Sanderson et al.

Figure 3.8. Illustration of the enzymatic reaction of luciferin in a microtiter plate. The reagent luciferase was added to the samples in a white microtiter plate. Immediately after, the reaction produces green light, proportional to the amount of ATP in the media. Retrieved from BioTek Glow Luminescence. 
Figure 3.9. ATP Standard Curve. The ATP standard reagent from the Bioluminescence Assay Kit HS II was diluted in series in a white microtiter plate. The luciferase reagent was added to the diluents and immediately after bioluminescence was measured with the multimode microplate reader, Cytation 5. The data was plotted in a log-log curve of ATP concentration $(\mathrm{mM})$ against Bioluminescence relative light units (rlu). Each point in the graph represents the mean value from 3 technical replicates and their standard deviation.

Figure 3.10. Basic setup of DLS measurement system with a particle size analyzer. The laser light is focused over the sample and the scattered light can be detected in several angles. Retrieved from Brookhaven Instruments

Figure 3.11. Schematic drawing of a transmission electron microscope. The electron light beam is accelerated down the microscope and the electromagnetic lenses focus the beam onto the specimen. The recording system shoots and produce the image on screen. Image retrieved and modified from Kuntsche et al. (2011).

Figure 3.12. Chemical structure of WST-8 and WST-8 formazan. Tetrazolium salt used to determine the number of live and dead skin cells by its reduction with dehydrogenase to produce WST-8 formazan.

Figure 3.13. Principle of the cell viability detection with the kit CCK-8. WST-8 receives two electrons from the viable cells to generate formazan. The electron mediator, 1Methoxy PMS, and the cofactors, $\mathrm{NAD}(\mathrm{H})$ and $\mathrm{NADP}(\mathrm{H})$, aid in the catalysis of the reduction reaction. Retrieved from Dojindo's cell counting kit-8 (1963).

Figure 4.1. Molecular structures of LA and GML under acidic (4.5) and near-neutral (7.4) $\mathrm{pH}$ conditions. LA has an ionizable carboxylic acid group that undergoes protonation/deprotonation depending on the $\mathrm{pH}$ condition. By contrast, GML is nonionic.

Figure 4.2. Overview of experimental strategy integrating biophysical and biological measurement approaches. 
Figure 4.3. Determination of critical micelle concentration for LA and GML in different $\mathrm{pH}$ environments. Peak wavelength is shown as a function of compound concentration in PBS solution for (A) LA at pH 7.4, (B) LA at pH 4.5, (C) GML at pH 7.4, and (D) GML at $\mathrm{pH}$ 4.5. Each point in the graph represents the average value of six scans $(n=6)$. Standard deviations (expressed as the error bars) are presented where applicable. The CMC value is defined as the highest test concentration before the first break point occurs.

Figure 4.4. Determination of the $\mathrm{pK}_{\mathrm{a}}$ value of lauric acid in water. Titration curves were established, measuring the solution $\mathrm{pH}$ as a function of the volume of $0.5,2.5$ and $5 \mathrm{mM}$ $\mathrm{NaOH}$ added to the solution. The $\mathrm{pH}$ of the inflection point was determined to be $\sim 7$ (corresponding to complete ionization of LA), and the $\mathrm{pH}$ value corresponding to half of the $\mathrm{NaOH}$ volume that was necessary to reach the inflection point was defined as the $\mathrm{pK}_{\mathrm{a}}$ value. Following this approach, the average $\mathrm{pK}_{\mathrm{a}}$ value obtained from the three curves was defined to be 5.8 .

Figure 4.5. QCM-D investigation of lauric acid treatment on supported lipid bilayers at $\mathrm{pH}$ 7.4. $\Delta f$ (blue line with squares) and $\Delta D$ (red line with triangles) shifts are presented as a function of time for (A) $4 \mathrm{mM}$, (B) $2 \mathrm{mM}$, (C) $1 \mathrm{mM}$, and (D) $500 \mu \mathrm{M}$ LA. The baseline values at $t=0$ min correspond to an SLB platform on the sensor surface. LA was added at $t=5$ min (arrow 1), and a buffer washing step was performed (arrow 2) after the measurement signals stabilized.

Figure 4.6. QCM-D investigation of lauric acid treatment on supported lipid bilayers at $\mathrm{pH}$ 4.5. $\Delta f$ (blue line with squares) and $\Delta D$ (red line with triangles) shifts are presented as a function of time for (A) $4 \mathrm{mM}$, (B) $2 \mathrm{mM}$, (C) $1 \mathrm{mM}$, (D) $500 \mu \mathrm{M}$, (E) $250 \mu \mathrm{M}$, (F) 125 $\mu \mathrm{M},(\mathrm{G}) 63 \mu \mathrm{M}$, (H) $31 \mu \mathrm{M}$, and (I) $16 \mu \mathrm{M}$ LA. The baseline values at $t=0$ min correspond to an SLB platform on the sensor surface. LA was added at $t=5 \mathrm{~min}$ (arrow 1), and a buffer washing step was performed (arrow 2) after the measurement signals stabilized. 
Figure 4.7. QCM-D investigation of glycerol monolaurate treatment on supported lipid bilayers at $\mathrm{pH}$ 7.4. $\Delta f$ (blue line with squares) and $\Delta D$ (red line with triangles) shifts are presented as a function of time for (A) $2 \mathrm{mM}$, (B) $1 \mathrm{mM}$, (C) $500 \mu \mathrm{M}$, (D) $250 \mu \mathrm{M}$, (E) $125 \mu \mathrm{M}$, (F) $63 \mu \mathrm{M}$, (G) $31 \mu \mathrm{M}$, (H) $16 \mu \mathrm{M}$, and (I) $8 \mu \mathrm{M}$ GML. The baseline values at $t=$ 0 min correspond to an SLB platform on the sensor surface. GML was added at $t=5 \mathrm{~min}$ (arrow 1), and a buffer washing step was performed (arrow 2) after the measurement signals stabilized.

Figure 4.8. QCM-D investigation of glycerol monolaurate treatment on supported lipid bilayers at $\mathrm{pH}$ 4.5. $\Delta f$ (blue line with squares) and $\Delta D$ (red line with triangles) shifts are presented as a function of time for (A) $2 \mathrm{mM}$, (B) $1 \mathrm{mM}$, (C) $500 \mu \mathrm{M}$, (D) $250 \mu \mathrm{M}$, (E) $125 \mu \mathrm{M}$, (F) $63 \mu \mathrm{M}$, (G) $31 \mu \mathrm{M}$, (H) $16 \mu \mathrm{M}$, and (I) $8 \mu \mathrm{M}$ GML. The baseline values at $t=$ 0 min correspond to an SLB platform on the sensor surface. GML was added at $t=5 \mathrm{~min}$ (arrow 1), and a buffer washing step was performed (arrow 2) after the measurement signals stabilized.

Figure 4.9. Microscopic observation of $2 \mathrm{mM}$ lauric acid-induced membrane morphological responses on supported lipid bilayers. (A) Image snapshots at various time points depict nucleation sites from which tubules grow upon LA treatment of SLB at pH 7.4. (B) Image snapshots at various time points depict nucleation sites from which buds grow and membrane phase separation occurs upon LA treatment of SLB at $\mathrm{pH} 4.5$. $t=0$ min corresponds to the introduction of $2 \mathrm{mM}$ LA solution into the measurement chamber. The scale bar is $20 \mu \mathrm{m}$.

Figure 4.10. Microscopic observation of $250 \mu \mathrm{M}$ lauric acid-induced membrane morphological responses on supported lipid bilayers. (A) Image snapshots at various time points depict the lack of membrane morphological response upon LA treatment of SLB at $\mathrm{pH}$ 7.4. (B) Image snapshots at various time points depict nucleation sites from which buds grow and membrane phase separation occurs upon LA treatment of SLB at $\mathrm{pH}$ 4.5. $t=0$ min corresponds to the introduction of $250 \mu \mathrm{M}$ LA solution into the measurement chamber. The scale bar is $20 \mu \mathrm{m}$. 
Figure 4.11. Microscopic observation of $500 \mu \mathrm{M}$ glycerol monolaurate-induced membrane morphological responses on supported lipid bilayers. (A) Image snapshots at various time points depict nucleation sites from which entangled tubes form, causing membrane budding and membrane lysis, upon GML treatment of SLB at pH 7.4. (B) Image snapshots at various time points depict nucleation sites from which buds grow upon GML treatment of SLB at $\mathrm{pH}$ 4.5. $t=0$ min corresponds to the introduction of $500 \mu \mathrm{M}$ GML solution into the measurement chamber. The scale bar is $20 \mu \mathrm{m}$.

Figure 4.12. MIC values for antimicrobial lipids against $C$. acnes bacteria. The MIC values for LA and GML at $\mathrm{pH} 7.4$ and 4.5 are reported from a minimum of three independent experiments using a two-fold dilution protocol. Standard deviations are negligible due to the assay format. The y-axis is presented in $\log _{2}$ scale for trend comparison.

Figure 5.1.Evaluation of glycolic acid as an antibacterial agent to inhibit $C$. acnes viability. (a) Molecular structure of glycolic acid in the nonionic (protonated) and anionic (deprotonated) states. The equilibrium ratio of glycolic acid molecules in the two states depends on the $\mathrm{pH}$ condition. (b) Experimentally determined lowest concentration of glycolic acid to fully inhibit $C$. acnes viability in different $\mathrm{pH}$ conditions. The $C$. acnes cell concentration was $1 \times 10^{6} \mathrm{CFU} \mathrm{mL}^{-1}$ and cell suspensions were incubated in glycolic acid solutions at different $\mathrm{pH}$ conditions for $1 \mathrm{hr}$ before agar plating to determine if glycolic acid treatment inhibited cell viability. Each data point is representative of three independent experiments. The boxed-in circles indicate $\mathrm{pH}$ conditions where an inhibitory concentration was not recorded for one of two reasons: the $\mathrm{pH}$ condition itself during the incubation step caused loss of C. acnes viability ( $\mathrm{pH}$ 2.5) or glycolic acid was inactive (i.e., not antibacterial) within the test range up to $200 \mathrm{mM}$ (pH 5.0 and 5.5). The dashed vertical line represents the $\mathrm{pK}_{\mathrm{a}}$ value of glycolic acid, which is around $\mathrm{pH} 3.83$.

Figure 5.2. $\mathrm{pH}$-dependent bactericidal activity of glycolic acid against $C$. acnes. The pHdependent reduction in viable $C$. acnes cell concentration is plotted as a function of glycolic acid concentration. The data correspond to (a) $\mathrm{pH} 2.5$, (b) $\mathrm{pH} 3.0$, (c) $\mathrm{pH} 3.5$, and (d) $\mathrm{pH}$ 
4.0. The $C$. acnes cell concentration was $1 \times 10^{6} \mathrm{CFU} \mathrm{mL}^{-1}$. The recorded $\mathrm{MBC}$ values are $12.5 \mathrm{mM}, 25 \mathrm{mM}$, and $50 \mathrm{mM}$ at $\mathrm{pH} 3.0,3.5$, and 4.0, respectively, and correspond to the lowest glycolic acid concentration at which no bacterial cell viability was detected (reported as ND, not determined). C. acnes cells were not viable after incubation in the $\mathrm{pH}$ 2.5 condition, whereas viability was maintained after incubation in the other test $\mathrm{pH}$ conditions as indicated by the $0 \mathrm{mM}$ glycolic acid control data for each case. Mean \pm standard deviation values are reported from $n=3$ experiments.

Figure 5.3. Effect of glycolic acid treatment on C. acnes cell viability. (a) Effect of glycolic acid concentration-dependent treatment on viable $C$. acnes cell concentration at $\mathrm{pH}$ 3.5. The $C$. acnes cell concentration was $1 \times 10^{8} \mathrm{CFU} \mathrm{mL} \mathrm{m}^{-1}$. The recorded MBC value was $25 \mathrm{mM}$, and corresponds to the lowest glycolic acid concentration at which no bacterial cell viability was detected (reported as ND, not determined). Mean \pm standard deviation values are reported from $n=3$ experiments. (b) Live-dead assay evaluation of glycolic acidtreated $C$. acnes cells at different glycolic acid concentrations by confocal laser scanning microscopy (CLSM) imaging. Green and red colors indicate live and dead C. acnes cells, respectively. Images are representative of three experiments. Scale bars: $100 \mu \mathrm{m}$.

Figure 5.4. Effect of glycolic acid treatment on bacterial cell membrane integrity. The concentration of extracellular ATP released from $C$. acnes cells was measured by bioluminescence assay. The $C$. acnes cell concentration was $2 \times 10^{8} \mathrm{CFU} \mathrm{mL}^{-1}$. Mean \pm standard deviation values are reported from $n=3$ experiments.

Figure 6.1. Overall experimental strategy. Bicelles were prepared by hydrating the lipid mixtures of DOPC and GML, and then processing the mixtures with the freeze-thaw-vortex cycling method. The resulting bicelles were evaluated for SLB formation and antibacterial activity.

Figure 6.2. QCM-D results of DOPC/GML bicelle adsorption onto silicon dioxide at different q-ratios. (A) Frequency and dissipation shifts, $\Delta \mathrm{f}$ (upper panel) and $\Delta \mathrm{D}$ (lower panel), respectively, were recorded as a function of time at $q=0.05$. The shaded areas 
represent the range of shifts an SLB. (B) Column graph of final shifts (upper panel for $\Delta \mathrm{f}$ and lower panel for $\Delta \mathrm{D}$ ) $v s$ lipid concentration for bicelles at $\mathrm{q}=0.05$. The dotted lines represent the typical values for an SLB. Corresponding results for bicelle adsorption at (CD) $q=0.25$ and $(E-F) q=2.5$.

Figure 6.3. Fluorescence microscopy imaging of the representative DOPC/GML bicelle adsorption cases at different q-ratios. Bicelles were added onto the glass surface at $t=0$ min and the fluorescence microscopy images were recorded for the entire adsorption process. Bicelle test conditions are $\mathrm{q}=0.05$ at (A) $0.031 \mathrm{mM} \mathrm{DOPC}$ and (B) $0.016 \mathrm{mM}$ DOPC, $q=0.25$ at (C) $0.5 \mathrm{mM}$ DOPC and (D) $0.063 \mathrm{mM}$ DOPC, and $\mathrm{q}=2.5$ at (E) 0.063 $\mathrm{mM}$ DOPC. Scale bars are $20 \mu \mathrm{m}$.

Figure 6.4. Characterization of bicelle-SLB interactions at different q-ratios. The GML concentration in DOPC/GML bicelles at different q-ratios was fixed at $0.125 \mathrm{mM}$. (A) Frequency and dissipation shifts, $\Delta \mathrm{f}$ (upper panel) and $\Delta \mathrm{D}$ (lower panel), respectively, were recorded as a function of time. The zero baselines, as denoted by dotted lines, correspond to pre-fabricated SLBs. Arrow (1) indicates the time point of bicelle injection and Arrow (2) buffer wash. (B) Column graphs of peak shifts (upper panel for $\Delta \mathrm{f}$ and lower panel for $\Delta \mathrm{D})$.

Figure 6.5. Biological activity evaluation of GML/DOPC bicelle at $q=0.25$. (A) CryoTEM micrographs of DOPC/GML bicellar formulation at fixed GML concentration of $0.125 \mathrm{mM}$ in the bicelle. The circular structure of the bicelles were detected in a size range of $100-600 \mathrm{~nm}$. Scale bars are $200 \mu \mathrm{m}$. (B) Antibacterial effect of DOPC/GML bicelles against $S$. aureus. The control bacterial cell concentration was $5 \times 10^{5} \mathrm{CFU} \mathrm{mL}^{-1}$ and $\mathrm{MBC}$ value was $0.125 \mathrm{mM}$, indicating that the lowest GML concentration in the bicelle at which no alive bacterial cells are observed (marked as ND, not determined). Mean \pm standard deviation was collected from $n=3$ experiments. (C) Live-dead bacterial viability evaluation of DOPC/GML bicelle-treated S. aureus cells at vary GML concentration in bicelles by confocal laser scanning microscopy (CLSM) imaging. The control bacterial cell concentration was $1 \times 10^{7} \mathrm{CFU} \mathrm{mL}^{-1}$. Live and dead bacterial cells were detected in green 
and red stain, respectively. Representative images are presented of triplicated experiments and scale bars are $50 \mu \mathrm{m}$.

Figure 6.6. Cytotoxicity testing of HaCaT cell line. The immortalized keratinocytes were treated with different concentration of the DOPC/GML bicelles and incubated for $72 \mathrm{hrs}$, after which the CCK reagent was added to the cells for $1 \mathrm{hr}$ incubation. The colorimetric culture media absorbance was measured with a plate reader at $450 \mathrm{~nm}$. The $0.5 \mathrm{mM} \mathrm{GML}$ concentration presented $6 \%$ of cell death, with $1 \mathrm{mM} \mathrm{23 \%}$ and with $2 \mathrm{mM} \mathrm{89 \% .} \mathrm{Mean} \pm$ standard deviation was collected from $n=3$ experiments. 


\section{Abbreviations}

AD

AHA

ArHA

ATP

ATR-FTIR

BHA

BSA

CFU

CLSI

$\mathrm{CMC}$

Cryo-TEM

DLS

DHPC

DMPC

DPPC

DOPC

EASI

EM

EUCAST

FA

FFEM

GA

GEA

GML

GRAS

HA

HPF

LA
Atopic Dermatitis

$\alpha$-Hydroxy Acids

Aromatic Hydroxy Acid

Adenosine Triphosphate

Attenuated Transmission Reflectance Fourier Transform Infrared

Spectroscopy

$\beta$-hydroxy acids

Bovine Serum Albumin

Colony Forming Unit

Clinical and Laboratory Standards Institute

Critical Micelle Concentration

Cryogenic Transmission Electron Microscopy

Dynamic Light Scattering

Dihexanoyl-phosphatidylcholine

Dimyristoyl-phosphatidylcholine

Dipalmitoylphosphatidylcholine

Dioleylphosphatidylcholine

Eczema Area and Severity Index

Electron Microscopy

European Committee on Antimicrobial Susceptibility Testing

Fatty Acid

Freeze Fracture Electron Microscopy

Glycolic Acid

Global Evaluation of Acne

Glycerol Monolaurate

Generally Recognized As Safe

Hydroxy acid

Hight Pressure Freezing Technique

Lauric Acid 


$\begin{array}{ll}\text { LB } & \text { Luria-Bertani } \\ \text { MBC } & \text { Minimum Bactericidal Concentration } \\ \text { MG } & \text { Monoglyceride } \\ \text { MH } & \text { Mueller-Hinton } \\ \text { MIC } & \text { Minimum Inhibitory Concentration } \\ \text { MRSA } & \text { Methicillin-Resistant Staphylococcus aureus } \\ \text { NMR } & \text { Nuclear Magnetic Resonance } \\ \text { PBS } & \text { Phosphate-buffered Saline } \\ \text { PHA } & \text { Polyhydroxy Acid } \\ \text { QCM-D } & \text { Quartz Crystal Microbalance-Dissipation } \\ \text { Rhodamine-DHPE } & \text { Lissamine Rhodamine B 1,2-dihexadecanoyl-sn-glycero-3 } \\ & - \text { phophaditylethanolamine } \\ \text { SANS } & \text { Small-Angle Neutron Scattering } \\ \text { SAXS } & \text { X-ray Scattering with Small Angle } \\ \text { SCORAD } & \text { Scoring Atopic Dermatitis } \\ \text { SLB } & \text { Supported Lipid Bilayer } \\ \text { TEM } & \text { Transmission Electron Microscopy } \\ \text { TEWL } & \text { Transepidermal Water Loss } \\ { }^{31 P ~ N M R ~} & \text { Phosphours-31 Nuclear Magnetic Resonance } \\ \end{array}$




\section{Chapter 1}

\section{Introduction}

Millions of people around the globe suffer from the two most common skin disorders: acne vulgaris and atopic dermatitis. Both diseases have no cure and most treatments involve the use of antibiotics. There is an imperative need to discover new alternative therapies that prevent the further increment of antibiotic-resistant bacteria and also alleviate the symptoms. In this sense, a potential solution are antimicrobial lipids in combination with glycolic acid. Antimicrobial lipids are directly found in skin and their job is to protect from the colonization of external bacteria. Their mechanistic behavior is by membrane disruption leaving a very small window for the development of resistance. Glycolic acid is a renowned hydroxy acid used to unclog pores and increase the cell turnover. Additionally, to improve the delivery, bicelles are a novel option to explore due to their verified effect on dermal applications. The desired outcome is an effective, yet mild therapy that can directly inhibit the bacteria triggering the diseases and at the same time treat the symptoms. Based on this vision, this chapter present a brief background of the research field and states the problem motivating the thesis. The proposed hypothesis and objectives of the project are detailed, along with the dissertation overview and novel findings. 


\subsection{Hypothesis/Problem Statement}

The most common skin disorders all around the globe are acne vulgaris and atopic dermatitis. They are characterized by inflammation, and multifactorial pathogenesis. Both diseases lack of an definite cure and the current treatments typically involve the use of antibiotics. In the light of the acquired antibiotic-resistance by several bacteria there is an urgent need to develop new antibacterial therapies. Acne vulgaris used to be a popular disorder among teenagers that now has extended its time frame to adulthood. It accounts for almost 50 million patients per year just in the United States ${ }^{1}$. The disease is characterized by the hyperproduction of sebum from sebaceous glands, leading to skin imperfections such as clogged pores and pimples along with inflammation around infected sites $^{2,3}$. The responsible bacteria are pathogenic strains of Cutibacterium acnes (formerly known as Propionibacterium acnes) ${ }^{4,5}$. The condition not only impacts the skin of the patient but its mental health. Severe acne is associated with depression, anxiety and poor self-esteem ${ }^{6}$. The second disorder, atopic dermatitis, also known as eczema, affects up to $25 \%$ of the children population and $3 \%$ of the adults worldwide ${ }^{7,8}$. Although the condition most often develops during childhood, it can occur at any stage of life ${ }^{8,9}$. Atopic dermatitis is characterized by inflammation, intense itch, red patches and dry scaly skin ${ }^{10}$. The bacteria associated with the conditions is Staphylococcus aureus, which colonizes lesioned and not-lesioned skin ${ }^{11-15}$. As with acne, atopic dermatitis also accounts for depression and physiological distress ${ }^{16}$. Despite of not being life threatening conditions both disease.

Acne vulgaris and atopic dermatitis, are prompted by bacteria imbalance. The human skin harbors numerous commensal microorganisms, including bacteria, fungi, viruses and even mites ${ }^{17}$. In healthy conditions, these microorganisms maintain a cooperative relationship between one another and the host skin surface, and further prevent colonization by pathogenic organisms ${ }^{18,19}$. In marked contrast, when this balance is disrupted, skin infections can be triggered by adjustments in bacterial populations, including establishing the dominance of invading bacterial species ${ }^{20}$. Opportunistic bacteria take advantage of the unbalanced environment, inducing changes in skin $\mathrm{pH}$ and causing pathogenic effects such as redness, inflammation and skin peeling ${ }^{4,21}$. 
In this sense the study of the skin microbiome gains great importance. The characterization and identification of the microbial communities thriving on skin provide an incredible opportunity to leverage the diagnosis, prognostic and treatment of skin conditions. The bio industry is and will continue to be revolutionized by the development of new therapies and treatments involving pro-, pre- and post- biotics; and any other compound which might regulate the skin microbiota. Worth noticing that the major obstacle for the development of microbiome based therapies is the complexity and stability of the microbial communities, which are dependent on the specific characteristics of the person age, sex, skin site and even weather conditions ${ }^{20}$. The skin microbiome resembles a finger print, no one has the same microbes or react the same when being in contact with other bacteria or fungi. Numerous antibacterial strategies have been devised to restore the optimal balance of skin bacteria, and reducing the density of $C$. acnes and $S$. aureus cells has been associated with improved skin conditions ${ }^{22,23}$. Typically, antibiotics that inhibit the growth of bacteria have been utilized to perform this task; however, the increasing rise of antibiotic-resistant bacteria highlights the need for new solutions that move beyond conventional antibiotics ${ }^{24-28}$.

A promising alternative to antibiotics are antimicrobial lipids which are surfactant-like molecules that destabilize bacterial cell membranes 29,30. Importantly, numerous antimicrobial lipids are widely used in the personal healthcare and food industries, and generally recognized as safe by the US Food and Drug Administration and other regulatory agencies worldwide ${ }^{31}$. Remarkably, antimicrobial lipids are naturally found on human skin surfaces and are a key part of the innate immune system ${ }^{32,33}$. They are found on the outermost layer of skin, and aid microbiome regulation by warding off invading bacterial species ${ }^{4}$. Before the invention of antibiotics in the 1940's, antimicrobial lipids were widely explored as an antibacterial solution, however, the high potency and scientific breakthroughs demonstrated by antibiotics at the time put a damper on the utilization of antimicrobial lipids ${ }^{34}$. Nowadays, with the recognition that we are entering a postantibiotic era there is renewed attention on antimicrobial lipids ${ }^{35,36}$. The two main classes of antimicrobial lipids are fatty acids (FAs) and their monoglyceride (MG) derivatives (produced by esterification of a fatty acid with a glycerol molecule). Both FAs and MGs 
are reported to exhibit potent antibacterial activity against numerous bacteria, with particularly high levels of activity reported for medium-chain saturated species ${ }^{37}$. It is currently understood that both classes of antimicrobial lipid kill bacteria by lysing bacterial cell membranes. Due to rapid lytic killing against the membrane target, there is a very high barrier to the emergence of drug-resistant bacteria and hence antimicrobial lipids can be used sustainably ${ }^{29}$. Within this scope, numerous researchers have confirmed that lauric acid (LA; C12:0) is the most inhibitory saturated FFA against Gram-positive bacteria ${ }^{37}$, and inhibits $C$. acnes and $S$. aureus ${ }^{22,38,39}$. The MG derivative of lauric acid is glycerol monolaurate (GML) and it exhibits even more potent inhibitory activity, albeit against a slightly different spectrum of bacteria, including $S$. aureus but not C. acnes ${ }^{30,37,40,41}$. Of note, the biophysical activities of these two antimicrobial lipids have been investigated in physiological (blood) pH conditions and clarifying how these antimicrobial lipids operate in skin-like environmental conditions, i.e. acidic $\mathrm{pH}$, in terms, terms of molecular selfassembly and corresponding interactions with phospholipid membranes and antibacterial activity, is important to identify promising drug candidates to carry forward.

Moreover, the development of antimicrobial lipids as novel solutions to treat C. acnes and $S$. aureus infection will further benefit from the development of drug cocktails comprised of FAs and/or MGs together with other classes of therapeutic molecules, especially those which treat disease symptoms ${ }^{24}$. Among suitable options, the alpha hydroxy acid glycolic acid represented a viable choice to renew skin and unclog pores ${ }^{42}$. It is a promising candidate to explore, especially in terms of understanding how its acidic effect on the solution environment and other more direct effects may further enhance the activity of antimicrobial lipids. It is worth remarking that the proposed therapy driving this research design strategy bear some resemble in composition to coconut oil, which is widely regarded as a "superfood". Coconut oil is principally composed of lauric acid and antioxidants and is extensively discussed as a potential solution to bacterial skin infections ${ }^{43}$. Going beyond natural compositions as found in the oil of the coconut and related materials, this thesis entails the systematic exploration of material building blocks, e.g., selecting the most desirable antimicrobial lipid, to optimize functionality while advancing scientific knowledge. Hence, identifying optimal combinations of antimicrobial lipids and additional 
therapeutic agents may lead to cocktails to treat $C$. acnes and $S$. aureus infections. To further improve the cutaneous delivery of the formulation with antimicrobial lipids, bicelles - nanostructures formed by amphiphilic molecules dispersed in an aqueous solution ${ }^{44,45}$ - are devised. Bicelles, also known as bicellar systems, are a new type of nanocarriers that have proved to be effective in dermal uptakes. Their phospholipid composition can be adjusted to meet the requirements in size, stability and compatibility with the lipids on skin. These carriers guarantee the local effect, appendage penetration and time controlled delivery ${ }^{46}$. Additionally, the pretreatment with bicelles boost the penetration of highly polar components, as glycolic acid. The phospholipid chosen for the preparation of the bicelles was dioleylphosphatidylcholine (DOPC) because of its strong percutaneous penetration capability and high morphological versatility ${ }^{47,48}$. The desired outcome is a well-characterized formulation to treat acne vulgaris and atopic dermatitis, providing high levels of antibacterial activity with reduction of disease symptoms as well.

In accordance, several key questions need to be answer:

- Which antimicrobial lipid — lauric acid and/or glycerol monolaurate — exhibits optimal antibacterial activity against $C$. acnes under acidic, skin-relevant $\mathrm{pH}$ conditions?

- Is glycolic acid capable to kill $C$. acnes under healthy skin conditions? Is this concentration value within a suitable range or is it too high? What is the mechanistic activity of glycolic acid against $C$. acnes bacteria?

- Is the effect of the antimicrobial lipid against bacteria reduced with a delivery carrier? How big does the bicelles becomes after the incorporation of the antimicrobial lipid?

- Does the bicelles protect and ensure the delivery of the antimicrobial lipid?

As stated, there is a critical need to develop innovative antibacterial solutions that improve treatment outcomes against acne vulgaris and atopic dermatitis by employing therapeutic molecules that reduce $C$. acnes and $S$. aureus cell counts, act against bacterial targets with high barriers to evolving drug-resistant mutations, and ameliorate disease symptoms such 
as skin inflammation. Antimicrobial lipids and glycolic acid - naturally sourced or chemically / enzymatically synthesized and possessing novel mechanistic profiles compared to classical antibiotics - are promising candidates to achieve these goals, however, successful translation of these molecules requires the characterization of the scope and potency of inhibitory activity against bacterial membrane targets and devising formulation strategies for optimal delivery. Guided by these opportunities, the overall hypothesis of this thesis is that the therapeutic activities of the antimicrobial lipids assembled as bicelles and glycolic acid can be employed in additive multifunctional combination to treat $C$. acnes and $S$. aureus infections.

\subsection{Objectives and Scope}

The scope of this research project was the characterization of antimicrobial lipids alone and assembled as bicelles in alliance with glycolic acid as potential candidates to treat $C$. acnes and $S$. aureus, bacterium implicated in acne and atopic dermatitis pathogenesis, respectively. In alignment with the hypothesis, an optimized and deep experimental characterization was done. The experimental approaches integrate the physicochemical, biophysical and biological methodologies to make connections and associative conclusions, and hence, provide a better solution. To accomplish the scope and answer the key questions from the hypothesis the following specific objectives were achieved:

Objective 1. Characterization of the antibacterial potency and environmental sensitivity of antimicrobial lipids, namely lauric acid and glycerol monolaurate, against $C$. acnes infection under skin-like settings. The followed experimental methodology began with fluorescence spectroscopy, to assess the molecular self-assembly of the antimicrobial lipids by means of the critical micelle concentration (CMC) in appropriate $\mathrm{pH}$ conditions (physiological, 7.4, and skin-like, 4.5). The $\mathrm{pK}_{\mathrm{a}}$ value of the fatty acid lauric acid was measured to further match it with its antibacterial behavior, dictated by the concept of higher antibacterial activity with protonated molecules. The value was not obtained for the monoglyceride, glycerol monolaurate, since its ionization state remains unaltered in all $\mathrm{pH}$ ranges. To study how antimicrobial lipids interact with phospholipids, main components 
of cell membranes, supported lipid bilayers (SLB) were used as a replacement for real-time evaluation of the morphological response. The concentration-dependent effect of the compounds was tracked with the change in mass and viscoelasticity of the SLB platform in a quartz crystal microbalance-dissipation (QCM-D). This move was done to dodge the major drawbacks of biological probes at the first stages of a project and limit the number of feasible permeating candidates. To complement the QCM-D experiments, fluorescence microscopy measurements were collected to visually observe the morphological changes. Together, these experiments are aimed at providing a deep insight of the disruptive behavior. Lastly, biological assays were performed to determine how $\mathrm{pH}$-dependent conditions affect the inhibitory activity of antimicrobial lipids against $C$. acnes.

Objective 2. Evaluation of the antibacterial and mechanistic activity of glycolic acid against $C$. acnes bacteria. The screening of the inhibitory concentration of glycolic acid against $C$. acnes was done with an antibacterial test, minimum inhibitory concentration (MIC). Next, enumeration essays to evaluate the concentration range at which glycolic acid kills $C$. acnes (by at least $99.99 \%$ ) in different $\mathrm{pH}$ conditions, was obtained with the minimum bactericidal concentration $(\mathrm{MBC})$ test. To confirm cell killing confocal microscopy imaging was performed to distinguish live and dead cells. The permeation effect of the cell membrane was recorded by monitoring the release of adenosine triphosphate (ATP), which is a sensitive marker of membrane damage.

It is important to remark that objective 1 and 2 were accomplished with $C$. acnes because, until the publication of the first two chapter of results, Chapter 4 and Chapter 5, glycerol monolaurate and glycolic acid were not tested against this bacteria under skin-like environmental conditions. The third objective involves the use of $S$. aureus because of the known information, faster growth and rapid assessment along with interest to explore such approaches against other skin-related bacteria and future opportunities stemming from this work are further covered in the last chapter of "Discussion and Future Work".

Objective 3. Development of bicelles for the protection, delivery and effect enhancement of the antimicrobial lipid on skin. Three bicelles with different lipid ratios of DOPC and 
glycerol monolaurate were design to select the best antibacterial treatment against $S$. aureus. To verify the membrane disruptive activity earlier described from the antimicrobial lipid a deep biophysical characterization was done over a SLB platform in Q-CMD, and images were taken with fluorescence microscopy. Of note, these set of experiments also aided to determine the possible formation of a SLB according to the ratio of long and short chain lipids. Based on the biophysical evaluations the best $\mathrm{q}$ ratio suggesting an anti-infective mechanism was chosen. The potency of the chosen bicelle was assessed by means of the bactericidal concentrations (MBC) testing and the bacterial counterstaining prove with the LIVE/DEAD kit. The technique supported the killing effect of the bicelle by taking images with the confocal microscope of the differently dyed live and dead bacteria. The morphological characterization of the bicellar sample was accomplished with dynamic light scattering and cryo-TEM. The first one to figure out the apparent size and dispersity of the bicelle, the second, to have a visual proof. At last, the cell proliferation and viability were tested over human immortalized keratinocyte cells (HaCaT) to prove the bicelles safeness.

\subsection{Dissertation Overview}

This thesis was organized in the following manner:

Chapter 1: "Introduction" states the problem that contextualizes the thesis. Describes in detail the proposed solution and the hypothesis rationale. It includes the scope, the key questions that arose from the hypothesis and the specific objectives to answer those questions.

Chapter 2: "Literature Review" reviews the up-to-date knowledge about acne vulgaris and atopic dermatitis. The origins, symptoms and therapy strategies for the diseases. It delved on the history of antimicrobial lipids, their spectrum, mode of action and experimental characterization. A summary of the application of antimicrobial lipids to the treatment of acne vulgaris an atopic dermatitis is provided. Successively, it outlines the hydroxy acids history and classification, making emphasis on glycolic acid and the reported experimental 
approaches. Regarding to the delivery vehicle, the chapter gives a general description of the bicellar systems and its use in skin-related applications. At last, it addresses the gaps in knowledge and underlines the outstanding questions that motivated the objectives of this thesis.

Chapter 3: "Experimental Methodology" lists the materials, materials synthesis and experimental techniques used for the characterization of the antimicrobial lipids, glycolic acid and bicellar systems. It gives a detailed explanation of the physical/chemical/biological principles behind each methodology and the utilized protocols.

Chapter 4: "Characterizing How Acidic pH Conditions Affect the Membrane-Disruptive Activities of Lauric Acid and Glycerol Monolaurate" assesses the effect of lauric acid and glycerol monolaurate treatment against SLBs and C. acnes bacteria in acidic $\mathrm{pH}$ conditions mimicking the skin environment. It provides great insight of potency and self-assemble of the antimicrobial lipids in accordance to the solution $\mathrm{pH}$. The biophysical results perfectly match with the biological outcomes gathered from the inhibitory tests.

Chapter 5: "pH-Dependent Antibacterial Activity of Glycolic Acid: Implications for AntiAcne Formulations" focuses on determining whether glycolic acid can effectively inhibit C. acnes, also under skin-like acidic conditions and how it works. The evaluations involves the protonation state of the molecule and how it can regulate its performance. The employed techniques belong to a biological background and aided to build upon the prior chemical knowledge of the molecule.

Chapter 6: "Engineering Phospholipid-Glycerol Monolaurate Bicellar Systems with Membrane-Disruptive Activity against Synthetic and Bacterial Cell Membranes" gauges the potency of glycerol monolaurate assembled as a bicelle prepared with DOPC and uses a more straightforward, yet of great relevance, bacterial model, S. aureus. This chapter encompass a multiple array of biological, physical and imaging techniques to draw connections and gain a deeper understanding of the assemble formed with the phospholipid 
and glycerol monolaurate, and its mechanistic activity. Additionally it provides an evaluation of the safe use of bicellar systems on skin cells.

Chapter 7: "Discussion and Future Work" comprises the key findings and pull together the three result chapters. It discusses the advances on the diseases treatment and the future research opportunities. Particular attention is placed on explaining how the outcomes verified the hypothesis, and ultimately, specific applications and solutions for the encountered drawbacks are proposed.

\subsection{Findings and Outcomes/Originality}

The findings disclosed in this research led to several novelties in the ground of dermatology and skincare. New alternatives for the reduction in bacteria cell density with negligible chances for antibacterial resistance were discovered. The mode of action and feasibility of the use in the acne treatment of a renowned compound in skincare was deciphered; and the encapsulation of an antimicrobial lipid inside an innovative nanocarrier was tried as a viable way to protect the compound, reach a target skin point and regulate the release. All those findings are reviewed below:

1. The CMC value of anionic fatty acids decreased under acidic conditions and in the case of nonionic monoglycerides remained the same. The CMC of lauric acid was almost $1 \mathrm{mM}$ at a physiological $\mathrm{pH}$ of 7.4, and it decreased 9 times under an acidic skin-like $\mathrm{pH}$ of 4.5 . The lessening in CMC occurred because the majority of lauric acid molecules were protonated under acidic conditions and hence micellar aggregation became more thermodynamically favorable. On the other hand, the CMC of glycerol monolaurate remained the same in both cases, proving the lack of $\mathrm{pH}$ dependence due to its nonionic character.

2. Different classes of antimicrobial lipids and solvent acidity values provoked different morphological responses in solution-phase model membrane platforms as SLBs. The anionic fatty acid, lauric acid, in acidic environments caused membrane phase separation, 
drastically different from the tubule formation that was observed in neutral settings. The nonionic monoglyceride, glycerol monolaurate, induced membrane budding in both $\mathrm{pH}$ settings, with the only difference in bigger size with acidic $\mathrm{pH}$.

3. The $\mathrm{pH}$ and molecular self-assembly of the antimicrobial lipids dictated the antibacterial activity profile. Unlike previous data, the fact that antimicrobial lipids were active against supported lipid bilayer at concentrations above the CMC value does not necessarily correlated with the minimum inhibitory concentration. Lauric acid was inhibitory against C. acnes bacteria only at physiological $\mathrm{pH}$ (7.4) despite of also being at a concentration above the CMC value in skin-like $\mathrm{pH}$ (4.5). On the contrary, glycerol monolaurate was active in both $\mathrm{pH}$ conditions at concentrations similar to its $\mathrm{CMC}$. The $\mathrm{pH}$ governed the protonation state and hence the self-assemble and antibacterial activity of the molecule. As supported, the molecular self-assembles capable to inhibit the bacterial cell growth were tubules, for anionic deprotonated fatty acids in neutral $\mathrm{pH}$, and membrane budding, for nonionic monoglycerides in all $\mathrm{pH}$ conditions.

4. Glycerol monolaurate was the most potent medium-chain saturated antimicrobial lipid against $C$. acnes bacteria in all $\mathrm{pH}$ conditions. The monoglycerides' nonionic protonated nature and its membrane budding behavior contributed to disrupt the bacterial cell membrane with lower concentration than lauric acid. This mechanistic activity has demonstrated to be a higher barrier to bacterial resistance and makes it a viable solution for acne infections.

5. Glycolic acid exhibited potent antibacterial activity against $C$. acnes, especially in acidic $\mathrm{pH}$ environments around $\mathrm{pH} 3$ to 4.5. Conventionally, glycolic acid is applied at relatively high concentrations in chemical peeling treatments. It was revealed that it could inhibit bacterial growth at concentrations as lower as $0.2 \%$ with $\mathrm{pH}$ conditions that were appropriate for over-the-counter formulation development.

6. The mechanistic behavior of glycolic acid against $C$. acnes bacteria was by disrupting its cell membrane and the potency relied on the solution $\mathrm{pH}$. The data indicated that 
glycolic acid was more active when it existed in the nonionic (protonated) form, which was consistent with a greater tendency of this form to partition into phospholipid membranes as compared to the anionic (deprotonated) form. Regarding to the membrane disruptive mechanism of action, it is significantly important because there is a documented higher barrier to bacterial resistance of membrane-active antibacterial drugs, like antimicrobial lipids, and the same probabilities might apply for glycolic acid as well.

7. The morphology of the bicelles prepared with DOPC and glycerol monolaurate with qratio of 0.25 was multilamellar vesicle and the size was $\sim 470 \mathrm{~nm}$. The diameter of the skin appendages is $530 \mathrm{~nm}$, thus the bicelles can penetrate and deliver the monoglyceride in the target point. Another hypothesis is that the bicelles can "sit" at the top of skin and slowly release the compound, protecting it from the environment.

8. The DOPC/GML bicelles with a q-ratio of 0.25 , ratio of DOPC to GML, are suitable for antibacterial applications but do not favor the formation of a SLB. The results revealed that a $\mathrm{q}=0.25$ does not produce a SLB on QCM-D and triggers pore formation according to fluorescence microscopy, which suggests potent antibacterial mechanism ${ }^{49}$. The reason is the higher amount of GML in comparison to the phospholipid DOPC. Conversely, a bicelles with a $\mathrm{q}=2.5$ self-assemble into a lipid bilayer due to the lower amount of GML and higher of the phospholipid.

9. The bactericidal concentration of glycerol monolaurate fabricated as bicelle increased and the cytotoxicity values with skin cells remained the same. The minimum inhibitory and bactericidal concentrations of GML alone against $S$. aureus bacteria were $0.063 \mathrm{mM}$ and $0.125 \mathrm{mM}$, respectively. However, when the antimicrobial lipid was prepared with the phospholipid DOPC the value changed to $0.125 \mathrm{mM}$ for $\mathrm{MIC}$ and $0.250 \mathrm{mM}$ for $\mathrm{MBC}$. These concentration are still within a good range and below the one from lauric acid alone of $1 \mathrm{mM}$ for MIC. The cytotoxicity tests with the bicellar systems and the monoglyceride alone showed a cell death percentage of less than $6 \%$, demonstrating the safeness of the delivery vehicle for cutaneous uptakes. 


\section{References}

1 Global Market Study on Acne Treatment: Inflammatory Acne Segment Anticipated to Account for 195 BPS Gain in Market Revenue Share During 2017 - 2025. Report No. PMRREP5279, 184 (Persistence Market Research, 2017).

2 Bickers, D. R. et al. The burden of skin diseases: 2004: a joint project of the American Academy of Dermatology Association and the Society for Investigative Dermatology. Journal of the American Academy of Dermatology 55, 490-500 (2006).

3 Bhate, K. \& Williams, H. Epidemiology of acne vulgaris. British Journal of Dermatology 168, 474-485 (2013).

4 Chen, Y. E. \& Tsao, H. The skin microbiome: current perspectives and future challenges. Journal of the American Academy of Dermatology 69, 143-155. e143 (2013).

5 Fitz-Gibbon, S. et al. Propionibacterium acnes strain populations in the human skin microbiome associated with acne. Journal of Investigative Dermatology 133, 21522160 (2013).

6 KHAN, M. Z., NAEEM, A. \& MUFTI, K. A. Prevalence of mental health problems in acne patients. Journal of Ayub Medical College Abbottabad 13, 7-9 (2001).

7 Nutten, S. Atopic dermatitis: global epidemiology and risk factors. Annals of nutrition and metabolism 66, 8-16 (2015).

8 Eichenfield, L. F. et al. Guidelines of care for the management of atopic dermatitis: section 1. Diagnosis and assessment of atopic dermatitis. Journal of the American Academy of Dermatology 70, 338-351, doi:10.1016/j.jaad.2013.10.010 (2014).

9 Hanifin, J. M., Reed, M. L. \& Group, I. W. A population-based survey of eczema prevalence in the United States. Dermatitis 18, 82-91 (2007).

10 Weidinger, S., Beck, L. A., Bieber, T., Kabashima, K. \& Irvine, A. D. Atopic dermatitis. Nature Reviews Disease Primers 4, 1, doi:10.1038/s41572-018-0001-z (2018). 
11 Kong, H. H. et al. Temporal shifts in the skin microbiome associated with disease flares and treatment in children with atopic dermatitis. Genome research 22, 850859 (2012).

12 Byrd, A. L. et al. Staphylococcus aureus and Staphylococcus epidermidis strain diversity underlying pediatric atopic dermatitis. Science translational medicine $\mathbf{9}$, eaal4651 (2017).

13 Huang, J. T., Abrams, M., Tlougan, B., Rademaker, A. \& Paller, A. S. Treatment of Staphylococcus aureus colonization in atopic dermatitis decreases disease severity. Pediatrics 123, e808-e814 (2009).

14 LEYDEN, J. J., MARPLES, R. R. \& KLIGMAN, A. M. Staphylococcus aureus in the lesions of atopic dermatitis. British Journal of Dermatology 90, 525-525 (1974).

15 Hanifin, J. M. \& Rogge, J. L. Staphylococcal infections in patients with atopic dermatitis. Archives of dermatology 113, 1383-1386 (1977).

16 Cheng, B. T. \& Silverberg, J. I. Depression and psychological distress in US adults with atopic dermatitis. Annals of Allergy, Asthma \& Immunology 123, 179-185 (2019).

17 Jain, S. in Dermatology: Illustrated Study Guide and Comprehensive Board Review 1-25 (Springer New York, 2012).

18 Oulas, A. et al. Metagenomics: tools and insights for analyzing next-generation sequencing data derived from biodiversity studies. Bioinformatics and biology insights 9, 75 (2015).

19 Grice, E. A. \& Segre, J. A. The skin microbiome. Nature Reviews Microbiology 9 , 244-253 (2011).

20 Grice, E. A. et al. Topographical and temporal diversity of the human skin microbiome. science 324, 1190-1192 (2009).

21 Grice, E. A. in Seminars in cutaneous medicine and surgery. 98-103 (Frontline Medical Communications).

22 Nakatsuji, T. et al. Antimicrobial property of lauric acid against Propionibacterium acnes: its therapeutic potential for inflammatory acne vulgaris. Journal of investigative dermatology 129, 2480-2488 (2009). 
23 Birnie, A., Bath-Hextall, F., Ravenscroft, J. \& Williams, H. Interventions to reduce Staphylococcus aureus in the management of atopic eczema. Cochrane database of systematic reviews (Online) 3, CD003871, doi:10.1002/14651858.CD003871.pub2 (2008).

24 Fitzpatrick, J. E. \& Morelli, J. G. Dermatology secrets plus. [electronic resource]. (Philadelphia, PA : Elsevier/Mosby, c2011.

4th ed., 2011).

25 Hoover, W. D., Davis, S. A., Fleischer, A. B. \& Feldman, S. R. Topical antibiotic monotherapy prescribing practices in acne vulgaris. Journal of Dermatological Treatment 25, 97-99, doi:10.3109/09546634.2013.852297 (2014).

26 Leyden, J. J., Del Rosso, J. Q. \& Webster, G. F. Clinical considerations in the treatment of acne vulgaris and other inflammatory skin disorders: focus on antibiotic resistance. Cutis 79, 9-25 (2007).

27 Ross, J. et al. Antibiotic-resistant acne: lessons from Europe. British journal of Dermatology 148, 467-478 (2003).

28 Dreno, B., Reynaud, A., Moyse, D., Habert, H. \& Richet, H. Erythromycinresistance of cutaneous bacterial flora in acne. European journal of dermatology: EJD 11, 549-553 (2001).

29 Kabara, J. J. Structure-function relationships of surfactants as antimicrobial agents. J Soc Cosmet Chem 29, 733-741 (1978).

30 Kabara, J. J. Antimicrobial agents derived from fatty acids. Journal of the American Oil Chemists' Society 61, 397-403 (1984).

31 Fu, X., Feng, F. \& Huang, B. Physicochemical characterization and evaluation of a microemulsion system for antimicrobial activity of glycerol monolaurate. International journal of pharmaceutics 321, 171-175 (2006).

32 Drake, D. R., Brogden, K. A., Dawson, D. V. \& Wertz, P. W. Thematic review series: skin lipids. Antimicrobial lipids at the skin surface. Journal of lipid research 49, 4-11 (2008).

33 Fischer, C. L. et al. The roles of cutaneous lipids in host defense. Biochimica et Biophysica Acta (BBA) - Molecular and Cell Biology of Lipids 1841, 319-322, doi:http://dx.doi.org/10.1016/j.bbalip.2013.08.012 (2014). 
34 Aminov, R. I. A brief history of the antibiotic era: lessons learned and challenges for the future. Frontiers in microbiology 1 (2010).

35 Alanis, A. J. Resistance to antibiotics: are we in the post-antibiotic era? Archives of medical research 36, 697-705 (2005).

36 Hammer, K. A. \& Carson, C. F. Antibacterial and antifungal activities of essential oils. Lipids and essential oils as antimicrobial agents. Chichester: John Wiley \& Sons, 255-306 (2011).

37 Kabara, J. J., Swieczkowski, D. M., Conley, A. J. \& Truant, J. P. Fatty acids and derivatives as antimicrobial agents. Antimicrobial agents and chemotherapy 2, 2328 (1972).

38 Yang, D. et al. The antimicrobial activity of liposomal lauric acids against Propionibacterium acnes. Biomaterials 30, 6035-6040 (2009).

39 Pornpattananangkul, D. et al. In vivo treatment of Propionibacterium acnes infection with liposomal lauric acids. Advanced healthcare materials 2, 1322-1328 (2013).

40 Yoon, B. K., Jackman, J. A., Kim, M. C. \& Cho, N.-J. Spectrum of membrane morphological responses to antibacterial fatty acids and related surfactants. Langmuir 31, 10223-10232 (2015).

41 Conley, A. J. \& Kabara, J. J. Antimicrobial action of esters of polyhydric alcohols. Antimicrobial agents and chemotherapy 4, 501-506 (1973).

42 Saint-Léger, D., Lévêque, J. L. \& Verschoore, M. The use of hydroxy acids on the skin: characteristics of C8-lipohydroxy acid. Journal of cosmetic dermatology $\mathbf{6}$, 59-65 (2007).

43 Tangwatcharin, P. \& Khopaibool, P. Activity of virgin coconut oil, lauric acid or monolaurin in combination with lactic acid against Staphylococcus aureus. Southeast Asian Journal of Tropical Medicine \& Public Health 43, 969-985 (2012).

44 Rodríguez, G. et al. Bicelles: new lipid nanosystems for dermatological applications. Journal of biomedical nanotechnology 11, 282-290 (2015).

45 Rodríguez, G. et al. in Soft Nanoparticles for Biomedical Applications 280-311 (Royal Society of Chemistry, 2014). 
46 Barbosa-Barros, L. et al. Effect of bicellar systems on skin properties. International journal of pharmaceutics 352, 263-272 (2008).

47 Yokomizo, Y. \& Sagitani, H. The effects of phospholipids on the percutaneous penetration of indomethacin through the dorsal skin of guinea pig in vitro. 2. The effects of the hydrophobic group in phospholipids and a comparison with general enhancers. Journal of controlled release 42, 37-46 (1996).

48 Pereira-Lachataignerais, J. et al. Effect of sodium dodecyl sulfate at different hydration conditions on dioleoyl phosphatidylcholine bilayers studied by grazing incidence X-ray diffraction. Langmuir 22, 5256-5260 (2006).

49 Valle-González, E. R. et al. Characterizing How Acidic pH Conditions Affect the Membrane-Disruptive Activities of Lauric Acid and Glycerol Monolaurate. Langmuir 34, 13745-13753 (2018). 


\section{Chapter 2}

\section{Literature Review}

The following chapter present the currently available information to support and justify the scope of the succeeding research. It ends with the outstanding questions that driven it. Since the objective is to design and characterize a formulation that overcomes the drawbacks faced by the current treatments for acne vulgaris and atopic dermatitis a wide description of the global diseases status, their profiles and the key factors that trigger the dermatoses are provided, including motivation for antibacterial treatment. Secondly, the motives for choosing the antimicrobial lipids, namely Lauric Acid and Glycerol Monolaurate, as an alternative option to kill pathogens without the undesirable secondary effects or even worst resistance. The third part includes the fundamental knowledge about glycolic acid due to the remarkable benefits it will provide in adjuvant combination therapies. Finally, a detailed review to a novel skin delivery method, bicelles, is given to point the followed path to the completion of the project. 


\subsection{Acne Vulgaris}

Acne vulgaris, popularly known as acne, is the most common skin disorder among teenagers. . Other types of acne next to vulgaris are rosacea, conglobata, fulminans, neonatal and infantile ${ }^{1}$. In the United States alone, there are 50 million patients annually. Worldwide, $95 \%$ of people will experience acne at least once in their lives, often chronically during adolescence and early adulthood. The average age of onset has decreased from 14-15 to 11-12 years old, owing to social and demographic adjustments ${ }^{2}$. Adults often suffer from the condition at later ages well, with routine cases now occurring up to the late 30's and beyond, in part due to evolving lifestyle patterns. This growing impact is reflected in rising market size, and acne treatments are estimated to run upwards of US\$3 billion dollars per year ${ }^{3,4}$ and a sharp increase is predicted in the coming years ${ }^{2}$. By and large, most acne treatments are over-the-counter products and focus on treating disease symptoms while more targeted strategies against disease-associated bacteria are needed urgently.

The responsible bacteria of this affection is Cutibacterium acnes (formerly known as Propionibacterium acnes), a gram positive, facultative anaerobic rod. It inhabits sebaceous sites such as the face, back and chest. It maintains a synergic relation with Staphylococcus epidermidis and Malassezia, a fungus. The optimal conditions for $C$. acnes growth are a temperature of $37^{\circ} \mathrm{C}$ with a $\mathrm{pH}$ range from neutral to slightly alkaline (approximately 67). It should be emphasized that $C$. acnes is found in both acne-affected and normal hair follicles, a fact which highlights the diversity of $C$. acnes strains and their differential effects on disease pathology ${ }^{5}$. Indeed, it has been found that the amount of C. acnes in individuals presenting with acne infection was nearly the same as that of individuals with healthy skin ${ }^{6}$. These findings reinforce the importance of developing antibacterial solutions to reduce pathogenic $C$. acnes cell counts, while recognizing the utility of lowcost natural materials with recognized safety profiles given the market dynamics as a whole. 


\subsubsection{Origins}

Acne originates due to the hyperproduction of sebum. This oily, nonpolar lipid substance is secreted by the sebaceous glands in skin pores. Its main function is to moisturize the skin and hair. However, when pathogenic strains of $C$. acnes are over-produced, they trigger hyper-keratinization, which is the second contributor to disease development. Keratinization causes thickening of the stratum corneum and occlusion of the sebaceous gland, which inhibits sebum release. It relates to the attachment of dead skin cells due to excessive sebum, which acts as a glue and drives cap formation. The third condition is the overgrowth of bacteria inside of the pore. C. acnes becomes trapped in the pore and begins colonization, leading to the generation of papules, pustules and nodules according to the severity of the case. The last condition is inflammation, noticeably seen with the appearance of skin swelling and redness. ${ }^{7}$

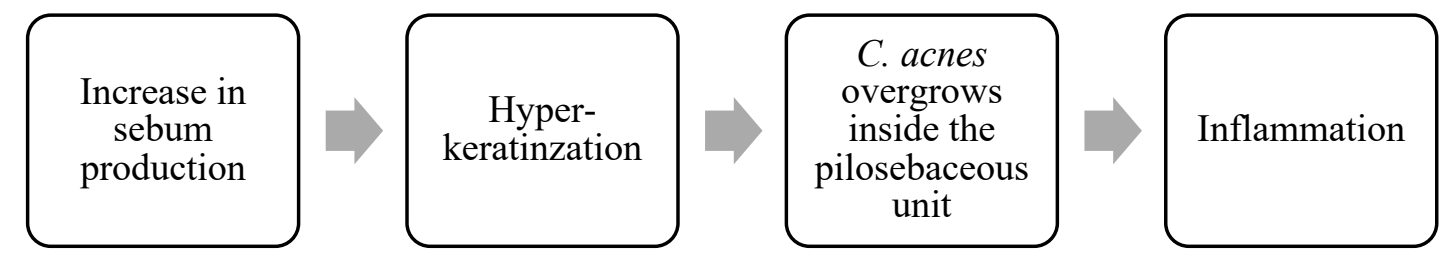

Figure 2.1. Contributors to acne development

It has been claimed that the disease origin occurs due to an imbalance within the skin microbiome and not from the hyperproduction of sebum ${ }^{6}$. This suggests that sebum excretion is under genetic control and the development of clinical lesions is instigated by the environment, including the growth rate of $C$. acnes. One particularly interesting experiment involving identical twins measured the rates of sebum excretion and the grade of acne severity. The data showed that the sebum rates were similar between siblings, but the grade of acne severity differed significantly ${ }^{8}$. This finding was pivotal to corroborate the importance of the skin microbiome and its regulation. One of the main issues influencing the skin microbiome is skin $\mathrm{pH}$, which under healthy conditions has an average value of 4.7 , and is in a range between $4-6^{9}$. When the skin becomes more alkaline, shifts 
in bacterial populations can occur that favor the colonization of undesired pathogens and the impairment of commensal bacteria. A proper acne treatment should allow for a smooth transition back to the normal flora, with particular focus on restoring the natural balance. To this end, such treatments would ideally deliver therapeutic molecules in skin-relevant, acidic $\mathrm{pH}$ formulations and preserve the resident microflora in sebaceous sites of the body. Taking into account all these factors, the current understanding of acne infection is that colonization and overgrowth of pathogenic strains of $C$. acnes is the causative event, while the overproduction of sebum and microbiome imbalance contribute to disease pathogenesis, but are not the cause per se.

\subsubsection{Symptoms}

The most frequent sites affected by acne are the skin regions with the greatest population of sebaceous glands, including the face, chest and back. The first evidence of acne is the formation of the comedon, black heads and white heads. The assessment of disease severity is conducted by the Global Evaluation of Acne (GEA), which is a 6-grade scale that provides a reliable evaluation of patient status (Table 2.1) ${ }^{10}$ 
Table 2.1. Global evaluation of acne disease status ${ }^{10}$

\begin{tabular}{|c|l|l|}
\hline Grade & Acne Status & Features \\
\hline 0 & $\begin{array}{l}\text { Clear. No } \\
\text { lesions }\end{array}$ & $\begin{array}{l}\text { Residual pigmentation and } \\
\text { erythema }^{\mathrm{A}} \text { may be seen. }\end{array}$ \\
\hline 1 & $\begin{array}{l}\text { Almost clear } \\
\text { with few lesions }\end{array}$ & $\begin{array}{l}\text { A few scattered open or closed } \\
\text { comedones } \text { B }_{\text {and very few }} \\
\text { papules }{ }^{\mathrm{C}} \text {. }\end{array}$ \\
\hline 2 & Mild & $\begin{array}{l}\text { Easily recognizable: less than } \\
\text { half of the face is involved. A } \\
\text { few open or closed comedones, } \\
\text { and a few papules and pustules }{ }^{\mathrm{D}} .\end{array}$ \\
\hline 3 & Moderate & $\begin{array}{l}\text { More than half of the face is } \\
\text { afflicted. Many papules and } \\
\text { pustules, and many open or } \\
\text { closed comedones. One nodule } \\
\text { may be present. }\end{array}$ \\
\hline 4 & Severe & $\begin{array}{l}\text { Entire face is involved, covered } \\
\text { with many papules and pustules, } \\
\text { open or closed comedones, and } \\
\text { rare nodules. }\end{array}$ \\
\hline 5 & Very Severe & $\begin{array}{l}\text { Highly inflammatory acne } \\
\text { covering the face along with the } \\
\text { presence of nodules. }\end{array}$ \\
\hline
\end{tabular}
A. Erythema: superficial reddening of the skin.
B. Comedon: clogged pore.
C. Papule: a small pimple or swelling on the skin.
D. Pustule: a small blister or pimple on the skin containing pus.
E. Nodule: a palpable, solid lesion, greater than $1 \mathrm{~cm}$ in diameter

\subsubsection{Treatment Strategies}

As mentioned above, numerous products exist to treat acne, including over-the-counter and prescription medicines. Acne therapy is usually delivered in topical forms, unless the status of the patient requires oral antibiotics. The reason to choose the cutaneous route as the first course of action is the immediate local effect and ease of administration (and hence compliance). Within this scope, only prescribed treatments are reviewed in this section, and cosmeceuticals are excluded because they focus on treating symptoms and not the disease itself . 
Table 2.2. Contributing steps in disease pathogenesis, and currently available treatments

\begin{tabular}{|c|c|c|c|c|}
\hline & $\begin{array}{l}\text { Increase in sebum } \\
\text { production }\end{array}$ & Hyper-keratinization & Bacteria growth & Inflammation \\
\hline $\begin{array}{c}\text { Contributing } \\
\text { factors }\end{array}$ & $\begin{array}{l}\text { Under androgen control } \\
\text { Decrease of sebaceous } \\
\text { gland activity }\end{array}$ & $\begin{array}{l}\text { Leads to comedon } \\
\text { development }\end{array}$ & $\begin{array}{l}\text { C. acnes colonizes } \\
\text { the pilosebaceous } \\
\text { unit }\end{array}$ & $\begin{array}{l}\text { Caused by } \\
\text { inflammatory } \\
\text { mediators } \\
\text { released into the } \\
\text { skin. }\end{array}$ \\
\hline $\begin{array}{l}\text { Treatment } \\
\text { available }\end{array}$ & $\begin{array}{l}\text { - Antiandrogens: } \\
\text { contraceptives, } \\
\text { corticosteroids and } \\
\text { flutamine }{ }^{11,12} \\
\text { - Oral antibiotic: } \\
\quad \text { Isotretinoin }{ }^{13}\end{array}$ & 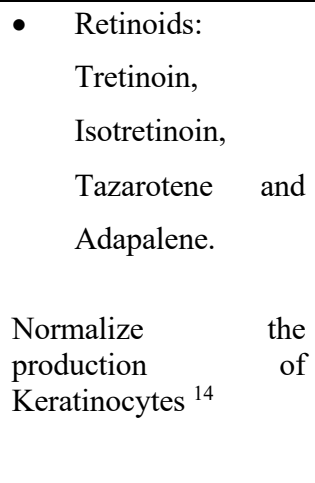 & $\begin{array}{l}\text { - Topical } \\
\text { antibiotics: } \\
\text { clindamycin, } \\
\text { erythromycin } 14 \\
\text { and azelaic acid }{ }^{15} \\
\text { Benzoyl } \\
\text { peroxide } \\
\text { Reduction in the } \\
\text { amount of } C \text {. } \\
\text { acnes on the skin }\end{array}$ & $\begin{array}{l}\text { - Topical } \\
\text { antibiotics: } \\
\text { clindamycin and } \\
\text { erythromycin. } \\
\text { - Dapsone }{ }^{18} \\
\text { Reduce redness } \\
\text { and swelling by } \\
\text { killing bacteria }\end{array}$ \\
\hline
\end{tabular}

\section{Treatments for mild to moderate acne}

The recommended prescribed treatment is a combination of a topical retinoid with benzoyl peroxide. The idea behind this combination therapy is to provide a broadly effective treatment to battle the disease origin and secondary effects, by reducing the $C$. acnes cell count and stopping keratinization, respectively. An oral or topical antibiotic, clindamycin or erythromycin, might be included depending on the severity of the case. The drawback is the development of bacterial resistance in the medium or long term, and hence monotherapy with antibiotics should be avoided ${ }^{7,14,19}$ 


\section{Treatment for severe acne}

Isotretinoin is the prevailing drug for treatment of severe conditions. It is an oral antibiotic that should be taken as a monotherapy for around four months in the prescribed doses. However, the side effects of this medicine are significant and include strong teratogenic effects, damage to the intestinal microflora and skin dryness. Pregnancy and blood tests must be conducted in parallel with routine monitoring of the patient to ensure treatment success. To this end, the FDA has designed a computer-based risk management program to restrict the consumption, prescription and sales of isotretinoin ${ }^{7,13}$. The recommended treatment after antibiotic intake is a topical retinoid with benzoyl peroxide as a maintenance therapy.

In summary, the suggested path to follow is the reduction of the $C$. acnes cell count with a topical antibiotic or benzoyl peroxide, combined with another remedy to treat the secondary effects and prevent continuous relapsing or emergence bacteria resistance. The adjuvant treatment, focused on collateral sequels, is varies depending on the most severe disease symptoms and chosen according to the patient's needs. At present, the most popular adjuvant approach involves the appliance of retinoid, which aids skin resurfacing and smoothing of skin lesions ${ }^{7,14}$.

\subsection{Atopic Dermatitis}

Atopic dermatitis (AD) is the most common inflammatory skin disease ${ }^{20}$. Also known as atopic eczema, it affects up to $25 \%$ of children and $2-3 \%$ of adults ${ }^{21,22}$. An average of $60 \%$ of the patients develop it within the first 12 months of birth, and $90 \%$ before the age 5 . Although the condition most often develops during childhood, it can occur at any stage of life 22,23 . Frequently the condition improves considerably at the time the children reach adulthood, but still 10-30\% will show symptoms ${ }^{24}$. A couple of studies reported the time frame for undergoing AD treatment, varying between 3-5 years and persisting sometimes up to 8 years ${ }^{25,26}$. The dermatitis occurs in larger proportion in male patients and has a higher rate of prevalence in people with a late onset of the disease ${ }^{27}$. Developed countries are the most affected, in the United States a 2007 survey resolute that approximately 17.8 
million persons, the majority without diagnosis, live with eczema ${ }^{23}$. Another study calculated to be $34.8 \%$ the mean percentage of income monthly spent on AD per patient, around $\$ 274$ USD $^{28}$. In 2013, the cost in treatment and lost of productivity of those who sought out their healthcare provider was $\$ 442$ million, \$314 million for medical cost and the rest for lost in productivity ${ }^{29}$.It is clear that $\mathrm{AD}$ is a heavy burden not only for the patients, but for their families and caregivers which look forward for superior therapies to improve the quality of life.

\subsubsection{Origins}

Atopic dermatitis is a multifactorial disease triggered by environmental factors in genetically susceptible people ${ }^{20}$. The origin implicates multiple chromosomes and until now 34 specific regions have been identified to harbor mutations associated with $\mathrm{AD}^{30-33}$. The chromosome with the higher linkage is $1 \mathrm{q} 21$, and the respective gene FLG ${ }^{34}$. The gene codes for the production of the protein profilaggrin, localized in the stratum corneum of the epidermis and in charge of harmonizing the amicrobial peptides (structural proteins) to create a strong skin barrier ${ }^{35}$. A mutation in the FLG gene results in the down regulation of the protein expression, and therefore weakening of the skin barrier. However, mutations in the FLG gene not necessarily cause AD, as found, only $20 \%$ of patients carry the mutation ${ }^{36}$. It is worth to highlight that as with any other genetic disease the family history is a leading factor ${ }^{22}$. The correlation incidence is $70 \%$ and the risk increase with the number of parents positive for the dermatitis ${ }^{37,38}$. A higher predisposition is typically associated to the mother ${ }^{39}$.

Other chromosomes highly associated with the condition are $5 \mathrm{q} 31$ and $5 \mathrm{q} 33$, which code for the immuno-system $\mathrm{T}$ helper cells type 1 and $2^{31,40}$. The cells produce small signaling proteins, called cytokines, that regulate inflammation. Patients with this mutation have a dominance of $\mathrm{TH}_{2}$ cells and overexpress cytokines, causing a decrease of the protein profilaggrin and other molecules in the skin ${ }^{34}$. In principle, all gene defects lead to abnormalities and permeability of the skin barrier, reflected in higher skin $\mathrm{pH}^{41}$, water loss ${ }^{42}$, irritability ${ }^{43}$ and of course, susceptibility to infections ${ }^{44,45}$. At the molecular level there 
is a reduction in the expression of differentiation-related structural proteins ${ }^{46}$, imbalance in protease-protease inhibitors ${ }^{47}$, altered composition of epidermal lipids ${ }^{48}$, diminished expression of tight junction proteins ${ }^{49}$ and shifts in the skin surface microbiota ${ }^{50}$. The last one, shifts in the skin microbiome, has been broadly studied and numerous publications verified the increased density of Staphylococcus aureus and decreased diversity of other bacteria in lesioned and not-lesioned skin of AD patients ${ }^{50-54}$. Indeed, an alternative strategy to tackle eczema is the reduction of the $S$. aureus bacteria which aggravates the symptoms ${ }^{55}$.

Lastly, the control of AD depends on the avoidance of triggering factor that may aggravate the condition. The most common factors are viral infections, food allergens, cosmetics, fragrance and extreme weathers ${ }^{27}$.

\subsubsection{Symptoms}

$\mathrm{AD}$ is a chronic relapsing, inflammatory skin disease characterized by intense itch, red patches with exudation, blistering and crusting at initial stages; and scaling, fissuring and thickening of the skin at advance stages ${ }^{20}$. It typically develops in 3 phases: acute, subacute and chronic. The first phase is characterized by vesicular, leaking and crusting eruptions. The second, presents swelling with dry, scaly and red plaques. The hallmark of chronic AD is the thickening of skin due to continuous scratching ${ }^{56}$. Severity scoring systems have been created as tools for measuring the AD symptoms, such as the Scoring Atopic Dermatitis $(\mathrm{SCORAD})^{57}$ and the Eczema Area and Severity Index (EASI) ${ }^{58}$. The two score intensity and extend of the lesion in different proportions and in general are the preferred instruments to measure patient-reported signs ${ }^{59}$. Classic $\mathrm{AD}$, manifest at babyhood in the bends of arms and legs and may progress to cheeks, forehead, scalp, chin and behind the ears. As the condition keeps escalating it may spread all over the body, except the diaper area and the nose. In adulthood, the face and neck are commonly involved ${ }^{60}$.

Eczema also causes some other chronic diseases, a phenomena known as "atopic march", which lead to the development of asthma, allergic rhinitis or food allergies ${ }^{61}$. The atopic march , as AD, can happen at any age and is linked to the predisposition for IgE-mediated 
response to stimuli ${ }^{62,63}$. On average $33.3 \%$ of atopic dermatitis patients develop asthma and $66.6 \%$ allergic rhinitis ${ }^{64,65}$. AD is also associate with mental disorders as attention-deficit, hyperactivity and speech disorder, as well as, sleep loss because of the itch and discomfort ${ }^{66-68}$. All over, numerous are the comorbidities associated with this skin dermatitis: bacterial infections, herpes and fungal infections ${ }^{27}$. As described before atopic dermatitis can persist throughout all life span and despite of not being life threatening it can greatly affect the quality of life and instigate anxiety and depression ${ }^{69}$. Hence, an early diagnosis and an effective treatment can significantly improve the overall condition.

\subsubsection{Treatment Strategies}

The diagnosis of atopic dermatitis is made by an extensive physical examination and history of the patient. However, it can be difficult and easily confuse with other similar diseases as seborrheic dermatitis and psoriasis ${ }^{70}$. To support the diagnosis, several sets of criteria have been developed. The Hanifin and Rajka criteria and the American Academy of Dermatology Consensus Criteria narrowed down the essential, common and associated features of the dermatitis ${ }^{71,72}$. The essential features in both classifications include intense itch; acute, subacute or chronic lesions; and chronic or relapsing symptoms.

The treatments for atopic dermatitis are classified according to the age and severity of the disease. The first line of action is the topical application, which has been successful for mild to moderate symptoms and useful as maintenance therapy for severe cases. Multiple over the counter products such as moisturizers, occlusive and humectants have demonstrated to strengthen the skin barrier; reduce itch and redness; and more importantly diminish the usage of prescribed therapies with multiple side effects ${ }^{73}$.

\section{Treatments for mild to moderate atopic dermatitis}

The treatments can be classified in two main categories: topical or phototherapy. Within the topical therapies corticosteroids, calcineurin inhibitors and cAMP-specific 3',5'cyclic PDE4 inhibitors are widely endorsed. Corticosteroids are the first line of antiinflammatories and lessen the recurrence of the disease when used alternatively, however they have multiple side effects, counting skin microbiota imbalance and decline in skin 
barrier function ${ }^{74}$. Calcineurin inhibitors are steroid-sparing anti-inflammatories which inhibit cutaneous $\mathrm{T}$ cell activation and proliferation ${ }^{75}$. Enough evidence support the repair of the epidermal barrier with their use ${ }^{76}$, unfortunately, the clinical efficacy is limited and the treatment expensive ${ }^{20}$. cAMP-specific 3',5'cyclic PDE4 inhibitors decrease the production of cytokines which trigger inflammation. Their mode of action is by inhibiting the intracellular enzyme with the same name, cAMP-specific $3^{\prime}, 5^{\prime}$ cyclic PDE4 ${ }^{77}$. The drug is prescribed for young patients with less than 2 years old, for sensitive body parts and as an alternative for the replacement of steroid treatments ${ }^{78}$. The other category, phototherapy, is the second line of treatment when AD cannot be controlled with topical measures. The duration of the treatment is between 4-8 weeks and the best outcomes have been obtained with a combination of narrow band UVB (NB-UVB) and medium-dose UVA 1 light ${ }^{79}$. As with the other therapies, it carries several risks as photodamage, dyspigmentation and greater chances for skin cancer ${ }^{80}$. The high price and associate costs should also be taken into account (Table 2.3.).

Table 2.3. Prescribed treatments for mild to moderate atopic dermatitis

\begin{tabular}{lll}
\hline $\begin{array}{l}\text { Topical } \\
\text { therapies }\end{array}$ & Corticoesteroids & $\begin{array}{l}\text { Divided in classes according to } \\
\text { vasoconstriction potency A }\end{array}$ \\
\cline { 2 - 3 } & Calcineurin inhibitors & $\begin{array}{l}\text { Tacrolimus } \\
\text { pimecrolimus }^{\mathrm{C}} \text { (cream) }\end{array}$ \\
\cline { 2 - 3 } & $\begin{array}{l}\text { cAMP-specific 3',5' } \text { cyclic PDE4 }^{\text {inhibitors }} \\
\text { Phototherapy }\end{array}$ & $\begin{array}{l}\text { Crisaborole ointment }^{\mathrm{D}} \\
\text { Use of ultraviolet B (NB-UVB) } \\
\text { and medium-dose ultraviolet A1 } \\
\text { (UVA1) light }\end{array}$ \\
\hline
\end{tabular}

A: Weidinger et al., $2018^{20}$, B: Nakahara et al., $2018^{75}$, C: Jensen et al., $2013^{76}$, D: Eichenfield et al., $2017^{78}$ and E: Garritsen et al., $2014^{79}$ 
Concomitant treatments for AD imply the use of antibiotics to reduce the S.aureus load in the lesions. The most common are gentamicin, fusidic acid and mupirocine by cutaneous administration ${ }^{81}$. Antiseptic lotions are common too.

\section{Treatment for severe atopic dermatitis}

The treatments for severe AD imply the use of systemic therapies, immunosuppressants or immunomodulator ${ }^{82}$. Systemic immunosuppressants are non-biological and comprise the drugs ciclosporin, azathioprine, methotrexate and mycophenolate mofetil. The most effective results have been achieved with ciclosporin, where $55 \%$ of the patients showed major recovery in less than 8 weeks ${ }^{83}$. Azathioprine and methotrexate display a $50 \%$ reduction of the symptoms after 20 weeks ${ }^{84,85}$. Mycophenolate mofetil, is the safest of the four, but it has a lower efficacy, thus, it is preferred as maintenance therapy ${ }^{86}$. Except for mycophenolate mofetil, the recommended usage of all these therapies is for no longer than 2 years ${ }^{87}$. On the other side, the only approved systemic immunomodulator is dupilumab. It is a human monoclonal antibody anti IL-4R $\alpha$ that blocks the signaling of IL-4 and IL-13 ${ }^{88}$. It is consider a rather safe treatment with only a few adverse effects: eye dryness and nasopharyngitis ${ }^{89}$.

\section{Alternative treatment for atopic dermatitis}

Alternative treatments for atopic dermatitis involve dietary restrictions, supplements, probiotics and prebiotics ${ }^{90}$. Patients with $\mathrm{AD}$ have not only a decreased skin barrier, but also a defective intestinal mucosa ${ }^{91,92}$. The best outcomes in prevention and reduction of AD severity involve bacterial diversity treatments, with specific probiotics, administered in mothers and children ${ }^{93}$. Lactobacillus rhamnosus GG shown to be effective in longterm prevention of $\mathrm{AD}{ }^{94}$. Lactobacillus paracasei speeded the intestinal microflora recovery ${ }^{95}$ and Lactobacillus salivarius aided in the modulation of cytokines release ${ }^{96}$.

\subsection{Antimicrobial Lipids}

The use of antibiotics to inhibit $C$. acnes and $S$. aureus is a mainstay treatment for acne vulgaris and atopic dermatitis. Nevertheless, their indiscriminate use topically or 
systemically has led to bacteria resistance. In the case of both pathogens, the situation has become a major clinical problem and been widely reported in numerous scientific publication ${ }^{97-101}$. The term antibiotic resistance is designated when the minimal inhibitory concentration (MIC) observed in laboratory test is greater than the concentration obtainable in vivo ${ }^{102}$. Hence, new treatment solutions are needed urgently to reduce C. acnes and $S$. aureus cell counts for therapeutic applications. A well-characterized and promising replacement for antibiotics consists of antimicrobial lipids, which display antibacterial activities ${ }^{103}$. Chief among them are fatty acids, which are found in the epidermis and are designed to protect against undesired bacteria and regulate the microbiome. In nature, fatty acids and their glycerol derivatives are found in milk and coconut oil ${ }^{104,105}$.

\subsubsection{Historical Origins}

The origin of the antimicrobial lipids study can be tracked down to the late 1880 's where the microbiologist Robert Koch discovered the powerful effect of soap to kill bacteria. He and his colleagues reported that fatty acids, a type of antimicrobial lipids and the main component of soaps, could inhibit the growth of Bacillus anthracis, a bacteria causing major health problems in human and livestock at his time ${ }^{106,107}$. Inspired by these findings multiple studies to understand the mechanism by which fatty acids inhibit the growth or kill bacteria arose ${ }^{106,108}$. In 1942, experiments conducted on skin and its appendages determined the existence of fatty acids in the surface and its role as part of the immune system. Burtenshaw et al showed that the fatty acids found in the skin surface had the ability to kill Staphylococcus aureus ${ }^{109}$. Nearly at the same time, the first antibiotic was discovered, penicillin, and changed forever the history of medicine ${ }^{110}$. Penicillin overshadowed the impact of fatty acids, not because of its limitations but rather because of its high potency to treat bacterial infections. As further explained in the upcoming section the mechanism of action of antimicrobial lipids consist of disrupting the bacteria cell membrane, conversely, antibiotics like penicillin block the assembly of peptidoglycans to form the bacteria cell wall. The antibiotic boom expanded to the treatment of animals and prophylactic applications, and even nowadays is considered the most reliable therapy for bacterial infections. However, the overuse of antibiotics came with a high price, leading 
the development of bacterial resistance and the current lack of effective drugs against certain infections. As widely acknowledged, society faces a post-antibiotic era ${ }^{111}$ and it exist an imperative need to develop new antimicrobial agents. A viable alternative are antimicrobial lipids, who have recently regained attention. Kabara et al are the forerunners of the rediscover and use of antimicrobial fatty acids ${ }^{103,112}$. In their studies it was demonstrated that medium chain unsaturated fatty acids showed greater efficacy against Gram-positive and negative bacteria 113,114 . Recent investigations have verified their applicability to also kill a wide range of viruses and fungi 115-117. On top of that, antimicrobial lipids are recognized as safe by the United States Food and Drug Administration and are used as food preservatives and cosmetic emulsifiers ${ }^{118}$. Their use extends to agriculture and husbandry ${ }^{119,120}$. From a biological point of view, their mode of action has demonstrated to be a higher barrier to bacterial resistance, since unlike antibiotics, it does not inhibit the synthesis of enzymes in the ribosomes, DNA or cell wall. To verify the postulate, bacteria cell cultures were grown with sub-lethal concentration of antimicrobial lipids for no less than one year, after which no signs of drug-resistance appeared $^{121}$.

\subsubsection{Classification}

Antimicrobial lipids are defined as single-chain lipid amphiphiles that interact with bacteria cell membranes and display antibacterial activity ${ }^{122}$. Among antimicrobial lipids, fatty acids are the most notable for their antifungal and bactericidal effect ${ }^{103}$. The chemical structure of fatty acids is composed by a hydrocarbon chain capped by a carboxyl group, and as the definition highlights they are of amphipathic nature. The hydrocarbon chain is hydrophobic and the carboxylic acid group is hydrophilic. The carboxylic group confers the molecule charge, either anionic or polar, depending on the $\mathrm{pH}$ of the aqueous solution. For instance, medium-chain saturated fatty acids have a $\mathrm{pK}_{\mathrm{a}}$ value of $\sim 5$, due to their anionic nature (deprotonated) in neutral $\mathrm{pH}$ conditions and nonionic (protonated) under acidic $\mathrm{pH}$ environments. The derivatives of fatty acids are monoglycerides and they also exhibit inhibitory activity against bacteria, albeit with a different spectrum of susceptible bacteria. Their chemical structure is a fatty acid bonded to a glycerol molecule with an 
ester group. Monoglycerides have a $\mathrm{pK}_{\mathrm{a}}$ value near 14 and remain nonionic (neutrally charged) or protonated in most $\mathrm{pH}$ settings.

The classification of antimicrobial lipids is based on the length of the hydrocarbon chain and degree of unsaturation. Natural antimicrobial lipids have an even number of carbon atoms, ranging from 4 to 28 . In the case of fatty acids, they are subdivided in short, medium and long chain. Short-chain fatty acids have less than 6 carbon atoms, medium between 6 and 12, and long more than 12 carbons. The degree of unsaturation refers to the type and number of bonds. Saturated fatty acids have only single bonds, unsaturated have at least one double bond in the hydrocarbon chain and polyunsaturated have more than 2 double bonds. Fatty acids with a saturated backbone are preferred to work with because of their higher chemical stability and lesser degree of oxidation ${ }^{123}$. The length of the carbon backbone, type and number of bonds and orientation (cis- or trans-) confer to each lipid amphiphile specific physicochemical properties. Making use of that knowledge, at the beginning of the 70's Kabara and coworkers evaluated multiple fatty acids according to their physicochemical properties to inhibit Gram positive and negative bacteria. As concluded, medium-chain saturated fatty acids and monoglycerides displayed the most potent inhibitory activity against Gram-positive bacteria, specifically C12, lauric acid (LA) and its monoglyceride, glycerol monolaurate (GML) $)^{112}$ (Table 2.4.). 
Table 2.4. Medium-chain fatty acids and monoglycerides

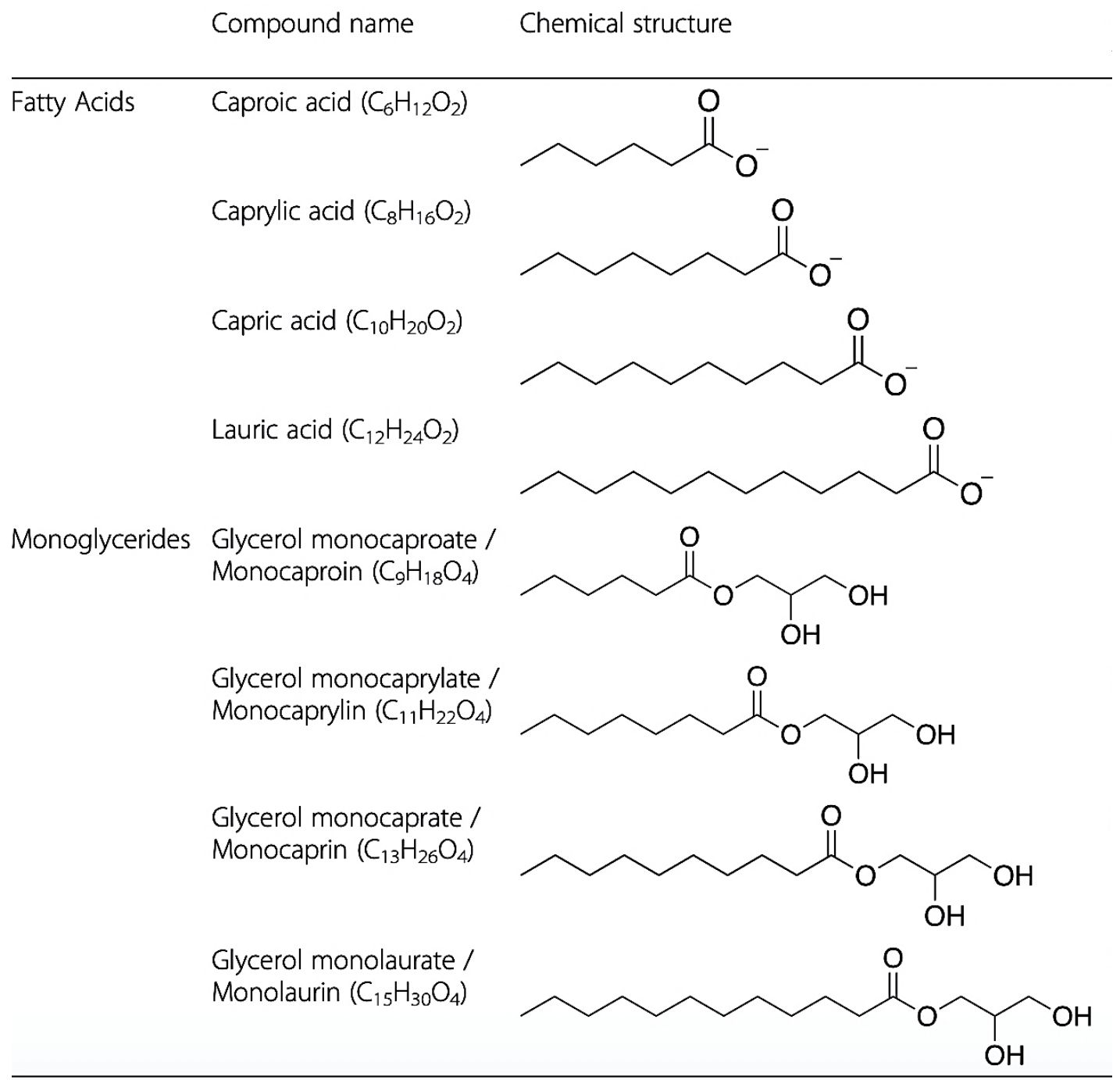

\subsubsection{Spectrum Antibacterial Activity}

Antimicrobial lipids exhibit potent inhibitory activity against a broad spectrum of bacteria, viruses, algae, and fungal pathogens, and found utility for anti-infective applications 115,119,124-126. As emphasized, lauric acid (C12) has the highest inhibitory effect among fatty acids, and its analogous monoglyceride, glycerol monolaurate, is even more potent. The largest amount of studies relating to antimicrobial lipids have been done by the biomedical and food science fields, showing the most promising outcomes with gram positive bacteria 127-131. The reason why gram positive bacteria are an easier target than gram negative is the 
their simpler single lipid bilayer cell membrane. Gram negative have more complex inner and outer membrane structures ${ }^{120}$. Relating to skin infections, numerous researchers confirmed the bactericidal properties of lauric acid against C. acnes ${ }^{132-135}$ and S. aureus $112,136,137$. For example, the potency of lauric acid and another good antimicrobial fatty acid, capric acid (C10) were compared ${ }^{138}$. Capric acid was effective and demonstrated good bactericidal and anti-inflammatory properties in vitro and in vivo, nevertheless, when compared with lauric acid, the performance of capric acid fell behind. In another investigation, the bactericidal concentration of lauric acid and benzoyl peroxide, which is the first-choice of treatment for moderate acne, were set side by side ${ }^{133}$. The in vitro experiment showed that the inhibitory concentration of lauric acid was 15-times lower than the value with benzoyl peroxide, these results matched for the 3 skin bacteria types used in the assay (C. acnes, S. aureus and S. epidermidis).

\subsubsection{Mechanism of Antimicrobial Activity}

The mechanism of antimicrobial activity of saturated fatty acids and monoglycerides targets the bacterial cell membrane and blocks the energy production. It acts in two different ways: (1) by increasing cell permeability and lysing the cell and (2) interrupting the electron transport chain and uncoupling the oxidative phosphorylation ${ }^{122}$. The first one, involves the amphipathic nature of the single chain lipids. They act as surfactants, destabilizing the phospholipid membrane from bacteria and forming pores, which leads to cell permeability and lately cell lysis. This disrupting effect can either inhibit the bacterial cell growth, namely bacteriostatic behavior, or kill the cell, bactericidal behavior. The bactericidal effect confer an advantage against resistance, owe to the almost immediate death ${ }^{139}$. A key feature for the lytic activity is the micellar state, similar to surfactants, the formation of micelles at lower concentrations increments the potency ${ }^{136,140,141}$. This explains why monoglycerides, forming micelles at very low concentrations, are more biologically potent than fatty acids. Going back to the second mechanism of action, interruption of the electron transport chain and uncoupling of oxidative phosphorylation, these two process are highly interconnected and involve the production of energy in the form of ATP. Fatty acids are capable to bind to the electron carriers and decrease the 
membrane potential and proton gradient. It compromises the membrane integrity and interrupts the production of energy, leading to cell death 142,143. As recognize, these mechanisms of action make it highly difficult for pathogens to mutate or become drug resistant 115,144 .

Worth to highlight, the interruption of the electron transport chain, oxidative phosphorylation and the blockage of membrane enzymes have been associated primary with unsaturated fatty acids (linoleic, oleic, palmitoleic acids) ${ }^{145-147}$, showing again that the physicochemical properties of the antimicrobial lipids determine their mode of action and bacteria spectrum. With this in mind, the characterization of the mechanistic behavior and killing profile should be tailored according to the type of antimicrobial lipid. The next section describes the microbiology and biophysical techniques used to specially characterize saturated fatty acids.

\subsubsection{Experimental Characterization of Antimicrobial Lipids}

Until recently, antimicrobial lipids were mainly studied by biological methods focused on determining the concentration-dependent efficacy to inhibit bacterial growth or kill bacteria. Minimum inhibitory concentration (MIC) and Minimum bactericidal concertation (MBC) tests are the standard techniques to assess antimicrobial agents and new drugs ${ }^{148}$. As their names state, the MIC is defined as the minimum concentration of a compound that inhibits the visible bacterial growth. The $\mathrm{MBC}$ is the minimum concentration value that kills at least $99.9 \%$ of the bacteria. Despite of the information about the potency and antibacterial spectrum, this traditional approach has been complemented by an explosion in biophysical-oriented studies that seek to characterize how antimicrobial lipids destabilize bacterial cell membranes, as well as gain insights into the underlying mechanistic determinants that can guide optimal selection of drug candidates for improve antibacterial strategies. Typically, electron microscopy (EM) techniques have been used to assess the bacterial cell membranes after treatment with antimicrobial lipids. EM facilitates the imagining of membrane morphological changes and intracellular damage ${ }^{149,150}$. In such cases, high concentrations $(\sim 5-10 \mathrm{mM})$ of test compound are incubated with bacteria, 
before a fixation step is applied and then the bacterial specimens are analyzed. Nonetheless, it is not possible to monitor real-time interactions, and atomic force microscopy approaches face the same challenge ${ }^{151}$ although the latter option carries the advantage of not requiring sample fixation prior to measurement.

To overcome these challenges, artificial membrane platforms such as solution-phase liposomes have enabled real-time monitoring of morphological changes caused by antimicrobial lipids, most commonly by dynamic light scattering ${ }^{152,153}$ or optical microscopy ${ }^{154,155}$ depending on the experimental system. Again, however, it remains difficult to resolve molecular-level interactions with these techniques and the main objective is to track gross morphological changes. To gain deeper molecular-level insights, supported lipid bilayers (SLBs) provide a versatile, two-dimensional model membrane platform that is compatible with a variety of surface-sensitive measurement techniques to investigate membrane morphological responses induced by antimicrobial lipids. ${ }^{156,157} \mathrm{~A}$ common experimental approach has been to utilize quartz crystal microbalance-dissipation (QCM-D) experiments to monitor in real-time the binding interactions and subsequent membrane morphological responses via changes in the mass and viscoelastic properties of the SLB platform, while fluorescence microscopy is employed to directly observe the type of membrane morphological response. Following this approach, Thid et al. first discovered that the DHA fatty acid induces tubule formation in SLB platforms composed of singlecomponent zwitterionic phospholipids, ${ }^{158}$ and this finding was later corroborated in another study that tested a range of environmental conditions. ${ }^{159}$ The membrane interactions of monocaprylate have also been studied using SLB platforms composed of more complex bacterial cell membrane extracts. ${ }^{151}$ In addition to profiling membrane morphological responses, SLB experiments have led to deep insights into the fundamental physicochemical behaviors driving these interactions.

Towards this goal, Yoon et al. utilized SLB platforms to distinguish the membrane morphological responses caused by fatty acids and monoglycerides. ${ }^{136}$ In agreement with the aforementioned works involving DHA, it was revealed that lauric acid (anionic) provoked the formation of elongated tubule structures, whereas glycerol monolaurate, 
(nonionic) caused membrane budding. The distinct membrane morphological responses were attributed to differences in the membrane translocation properties of anionic versus nonionic compounds, ${ }^{160}$ and corresponding effects on membrane strain ${ }^{161}$. Outstandingly, it was demonstrated that the membrane-destabilizing activities of LA and GML are dependent on their respective critical micelle concentration (CMC) values, and GML was more potent than LA on account of a lower CMC value, which could be explained by the tendency of nonionic compounds to aggregate more favorably than otherwise similar, anionic compounds. ${ }^{136}$ It was also identified that the CMC values of LA and GML are correlated with their respective minimum inhibitory concentration (MIC) values against Staphylococcus aureus, offering a link between membrane-disruptive and biological activities. Similar membrane-destabilizing effects were observed in cholesterol-enriched SLBs, reinforcing that membrane morphological responses provide insight into the different mechanisms by which antimicrobial lipids interact with membranes. ${ }^{162}$ Furthermore, it was shown that another fatty acid and monoglyceride pair, capric acid (C10:0) and monocaprin, follows these trends in the type and concentration-dependence of membrane morphological responses. ${ }^{156}$ Collectively, these findings established that the micellar aggregation properties of fatty acids and monoglycerides are intimately connected with how they interact with phospholipid membranes and corresponding biological activities, and it is therefore important to characterize the membrane-activities of antimicrobial lipids in appropriate environmental conditions.

Regarding to the activity of antimicrobial lipids on skin surfaces, where they play an important regulatory role 163 and exogenous administration of antimicrobial lipids represents a viable therapeutic strategy ${ }^{134,164,165}$. From a chemical perspective, the problem is interesting because the skin surface is an acidic environment, with $\mathrm{pH} 4.7^{9}$, and there is enough evidence showing that ionizable fatty acids exhibit increased antibacterial activity in the same conditions ${ }^{166}$. Similar trends have been reported for other anionic surfactants as well ${ }^{167,168}$. On the other hand, the antibacterial potency of monoglycerides, which do not possess ionizable functional groups in physiologically relevant $\mathrm{pH}$ conditions, does not depend on solution $\mathrm{pH}^{166,169}$. Taking into account that the acid dissociation constant of fatty acids lies in the acidic regime, these findings suggest that the ionization state of an 
antimicrobial lipid may play an important role in regulating the potency and scope of antibacterial activity and, as a corollary, the spectrum of membrane morphological responses. To date, the existing SLB platform studies aimed at distinguishing membrane morphological responses induced by antimicrobial lipids were conducted only under nearly neutral $\mathrm{pH}$ conditions. Chapter 4 , focused on elucidating this conundrum and assessed the performance or lauric acid and glycerol monolaurate under skin $\mathrm{pH}$.

\subsubsection{Applications to Acne Vulgaris Treatment}

The efficacy of lauric acid to kill $C$. acnes has been reported with demonstrated in vitro and in vivo activity ${ }^{132-135,138}$. LA is a minor component of the skin sebum and form part of the innate immune system ${ }^{170}$. The antimicrobial activity, as well as complementary skin cytotoxicity studies, corroborate that LA has high antibacterial activity against $C$. acnes and topical formulations are safe against human skin cells. Experiments involving other medium-chain saturated fatty acids, as capric acid, and usual medication for acne vulgaris, such as benzoyl peroxide, indicated that these compounds have lagged behind ${ }^{133,138}$. In addition, the potential of LA has been boosted by encapsulating it in liposomal bilayers, to increase permeation and delivery through the almost impenetrable first layers of skin ${ }^{132,134}$. Alternative strategies, such as nano-sized micelles ${ }^{171}$ and solid lipid nanoparticles ${ }^{172}$ loaded with LA also displayed positive result by inhibiting the growth of $C$. acnes and other skin bacteria. Apropos cosmetic formulations, lauric acid is commonly used for liquid soaps and cleansers. It provides a sweet smell and improves the feel of use ${ }^{173}$. The anionic nature of $\mathrm{C} 12$ makes it a good emulsifier and surfactant when used in moderation or in combination with a nonionic agent, otherwise, it can be too harsh for sensitive skin.

With respect to GML, monoglycerides have been reported as the safest group of chemicals known and, in fact they are food substances. Glycerol monolaurate has a high skin affinity, with low or no skin irritation, and is highly recommended for cosmetic formulations and pharmaceuticals. Due to its hydrophilic-lipophilic nature it acts as an emulsifier, providing stability and longer shelf life. GML is a multifunctional additive that might be employed as preservative, emulsifier, emollient, thickening agent, deodorant and transdermal agent 
174. In terms of its anti-infective activity, several publications have demonstrated its effectiveness against gram-positive bacteria. Bergsson et al. showed the effects of LA and GML to kill S. aureus. Superior activity of GML was identified, which was subsequently studied by electron microscopy to identify the mode of action. As expected, the bacteria were killed by disintegration of the cell membrane ${ }^{175}$. Schlievert et al. also tested LA and GML against biofilms and planktonic cultures. They concluded that GML is bactericidal for aerobic and anaerobic gram-positive bacteria in broth and biofilms, and verified that GML exhibits greater bactericidal activity than LA across the different bacterial conditions 144. A more recent study highlighted the potency of GML by testing its biophysical properties and correlating these results with antibacterial evaluation against $S$. aureus ${ }^{136}$. The concentration values leading to micelle formation of LA and GML agreed well with the MIC values of the two membrane-active compounds to inhibit S. aureus. In accordance with this mechanistic rationale, GML forms micelles at a lower concentration value, meaning greater potency for antibacterial activity.

Hence, the effects of GML as a potential strategy against acne deserve attention. As a matter of fact, the only reported concentration of GML used in a topical treatment, as a leave-on formulation, is $2 \mathrm{~g} / \mathrm{L}$, and it is used in combination with zinc pyrithione ${ }^{176}$. This treatment is a commercial product and the antibacterial properties of GML alone or in formulation against $C$. acnes have never been scientifically reported to date. According to the positive findings with LA ${ }^{133}$ and the promising features of the monoglyceride in cosmetic applications ${ }^{177}$, GML suggested that it might be an even more potent option against $C$. acnes.

\subsubsection{Applications to Atopic Dermatitis Treatment}

For the treatment of atopic dermatitis, lauric acid has been used to reduce the cell count of S. aureus, in vitro and in vivo ${ }^{133}$. Multiple strains of the bacteria, including MethicillinResistant Staphylococcus aureus (MRSA), have been assessed and LA has proven to be the most potent saturated fatty acid ${ }^{178}$. These outstanding inhibitory results have also been seen with LA encapsulated within a delivery vehicle ${ }^{172}$. In addition, LA has been advised for the formulation of moisturizers for $\mathrm{AD}$, to repair the skin barrier and fill the epidermal 
cracks ${ }^{179}$. Within this context, Verallo-Rowell et al assessed the moisturizing and antiinfective properties of virgin coconut and olive oil in eczema skin lesions colonized with $S$. aureus ${ }^{180}$. It was discovered that coconut oil not only protects the skin barrier, but inhibits the growth of the pathogen with the monoglyceride produced from hydrolyzation of the oil. The lipases produced by $S$. aureus breakdown the triglycerides and produce monoglyceride molecules, namely glycerol monolaurate. The same results were reported by other authors, which also noticed a better antibacterial activity with hydrolyzed virgin coconut oil ${ }^{181}$. All of these findings perfectly match with the fact that the primary source of lauric acid and glycerol monolaurate in nature is coconut oil, and the extraction of this compounds is through lipase-catalyzed glycerolysis ${ }^{182}$. As evidenced, GML is even more effective for ameliorating the symptoms of $\mathrm{AD}$, no mattering the lipases produced by $S$. aureus. Ling et al hypothesized that the alkylglycerol ether from GML, 1-O-Dodecyl-racglycerol (DDG), would be more effective against AD bacteria due to its supposed stability to lipase degradation ${ }^{183}$. The results proved them wrong and GML was more topically potent in vivo. Some recent studies backup not only the topical application but the oral ingestion too ${ }^{184,185}$. Infant formulas infused with GML prevent atopic diseases to occur later in life ${ }^{186}$ and the consumption of breast milk, naturally containing the monoglyceride, improves the recurrence ${ }^{187}$. Therefore, topical skin creams and oral supplements containing GML can be considered for treating all kinds of dermatitis.

As a corollary, the inhibitory results of $\mathrm{C} 12$ and its monoglyceride against Gram positive bacteria motivated the selection of glycerol monolaurate in this thesis for the assessment with Cutibacterium acnes. The use of GML against $C$. acnes was not evaluated until the investigation of the first part of this research, making it an innovative option. Since the results with lauric acid had already been published by other authors, they were recreated and used as a comparative. Regarding to the treatment of atopic dermatitis, the bacteria Staphylococcus aureus is inhibited with both lipid amphiphiles, hence, some experiments were conducted just as control. 


\subsection{Hydroxy Acids for Skincare}

As seen with acne, conventional therapies focus on combination treatment and devising multifunctional strategies for using antimicrobial lipids would be advantageous. While combination therapies are valued for treating the infectious bacterium along with disease symptoms, the potential for synergy is even more desirable and this section reviews the use of an organic acid, glycolic acid, with complementary properties and discusses mechanistic details of relevance to developing synergistic formulations. As aforementioned in the scope of the project, the goal is to develop a formulation which targets the origins and the secondary effects utilizing only one product. As an outcome, it might emerge as a suitable replacement to avoid antibiotic resistance. In the subject of atopic dermatitis, cutaneous formulation with organic acids have proven to improve the disease, by lowering $\mathrm{pH}$ and boosting the production of ceramides. The dry outer most layers of the skin are detached, favoring the hydration of epidermal cells. For all of these motives, it was postulated that organic acids, specifically glycolic acid, can be pour into antimicrobial lipid formulations or any other prescribed treatments, as a booster or synergistic element to enhance the delivery, penetration and potency.

\subsubsection{Historical Origins}

Hydroxy acids (HAs) are organic acids having one or more hydroxyl group attached directly to the hydrocarbon chain ${ }^{188}$. Hydroxy acids are found in nature in plants, fruits and milk. Their extraction can be via chemical or enzymatic synthesis, the latest being the most eco-friendly with less amount of by-products. The use of hydroxy acids can be tracked down to the beginnings of human civilization, where Samarians cured joint pain and inflammation with willow leaf ${ }^{189}$ and Egyptian women bathed themselves in sour milk to beautify the skin ${ }^{190}$. In the traditional Indian medicine the use of hydroxy acids is a common practice to treat skin condition as anti-aging, anti-inflammatory and analgesic ${ }^{191}$. In the XIV century, aristocrat French women washed their faces with old wine to eliminate redness and obtain a younger look ${ }^{192}$. However the scientific discovery of hydroxy acids was in the 1800 's. The chemist Auguste Laurent named glycolic acid the compound produced from the synthesis of gelatin sugar ${ }^{193}$. Later in 1851, Adolph Strecker prepared 
for the first time glycolic acid with the chemical reaction of hippuric acid, nitric acid and nitrogen dioxide ${ }^{194}$. Since then, the application of hydroxy acids expanded to the food, pharmaceutical and cosmetic industry ${ }^{195}$. In pharma, they are being synthetized for the production of antitumor agents from mandelic acid ${ }^{196}$, for the manufacture of anti-obesity medications and for semi synthetic penicillin ${ }^{197}$ and cephalosporines ${ }^{198}$. In the case of skincare, hydroxy acids revolutionized the industry since their appearance almost 40 years ago ${ }^{199}$. They are used for the treatment of photoaging, pigmentation, psoriasis, acne and eczema ${ }^{195,200}$. Nowadays it is known that the use of willow leaf provided relief due to the salicylic acid, the sour milk exfoliated skin because of the lactic acid, and the old wine decreased redness and provided plumpness because of the tartaric acid.

\subsubsection{Classification}

Hydroxy acids are classified according to the position of the hydroxyl group on the hydrocarbon chain. The four main categories of HAs are: $\alpha$-hydroxy acids (AHAs), $\beta$ hydroxy acids (BHAs), polyhydroxy acids (PHAs) and aromatic hydroxy acids (ArHAs) 188. Alpha and beta hydroxy acids are the two main classes utilized for skincare products. AHAs are carboxylic acids with the hydroxyl group bonded to the $\alpha$ carbon of the skeleton 195. They are polar and highly soluble in aqueous solvents. AHAs aid cellular renewal, by detaching the dead cells from the stratum corneum and promoting hyaluronic acid and collagen synthesis ${ }^{199}$. It has been proven that the topical application of AHA lessens the concentration of calcium ions leading to the breakage of desmosomes. Desmosomes are keratin patches that glue together corneocytes, when corneocytes are no longer attached they fall and encourage cell turnover ${ }^{200}$. Alpha hydroxy acids are able to penetrate through the epidermis and dermis, hence, they are capable to provide a smoother complexion, with less wrinkles, fade blemishes and improve the hydration and barrier function of skin ${ }^{201}$. The most common AHAs used in skincare have been listed in Table 2.5. Beta hydroxy acids are organic acids with the hydroxyl functional group separated by two carbons ${ }^{195}$. In contrast to AHAs, BHAs are soluble in lipids and thus can penetrate deeper into the skin. They are appropriate for the treatment of oily skin, since they can easily unclog pores and reduce inflammation ${ }^{202}$. BHAs have proved to be less irritant than AHAs, nonetheless their 
concentration in skincare products is normally between $1-2 \%{ }^{203}$ and $6 \%$ is the highest 199,204. The most common BHAs are salicylic acid and its derivative lipohydroxy acid, which produces the same good results with even higher tolerability ${ }^{205}$.

Table 2.5. Alpha hydroxy acids for skincare ${ }^{201}$

\begin{tabular}{|c|c|c|c|}
\hline Name & Chemical formula & Acidity [pKa] & Source \\
\hline Glycolic acid & $\mathrm{C}_{2} \mathrm{H}_{4} \mathrm{O}_{3}$ & 3.83 & Sugarcane \\
\hline Lactic acid & $\mathrm{C}_{3} \mathrm{H}_{6} \mathrm{O}_{3}$ & 3.86 & Fermented milk products \\
\hline Malic acid & $\mathrm{C}_{4} \mathrm{H}_{6} \mathrm{O}_{5}$ & 3.40 & Apples \\
\hline Tartaric acid & $\mathrm{C}_{4} \mathrm{H}_{6} \mathrm{O}_{6}$ & 3.22 & Fermented grapes \\
\hline Ascorbic acid & $\mathrm{C}_{6} \mathrm{H}_{8} \mathrm{O}_{6}$ & 4.10 & Fruits \\
\hline Citric acid & $\mathrm{C}_{6} \mathrm{H}_{8} \mathrm{O}_{7}$ & 3.09 & Citrus fruits \\
\hline Mandelic acid & $\mathrm{C}_{8} \mathrm{H}_{8} \mathrm{O}_{3}$ & 3.41 & Bitter almonds \\
\hline
\end{tabular}

\subsubsection{Glycolic acid}

Glycolic acid (GA) forms part of the alpha hydroxy acids (AHAs) family which are conformed by a carboxylic acid and a terminal hydroxy group. In the particular case of GA the two groups are linked with a carbon, being the smallest AHA (Figure 2.2.). Its size gives a head-start, since the penetration rate is higher in comparison with larger molecules 206. GA is extracted from fruits and sugar cane. It displays remarkable features improving skin conditions, positioning its main niche in anti-aging. GA reduces lines and wrinkles by allowing cell renewal and improving moisturizing levels ${ }^{207}$. Aging is mainly caused by exposure to UV A and B rays for long periods, an effect known as photo damage ${ }^{208}$. Skin pigmentation, wrinkles, loss of elasticity, less plumpness and degradation of collagen are the visible sequels ${ }^{209}$. All the aforementioned concerns had been reportedly counter act by GA by enhancing the production of hyaluronic acid and collagen, which are indispensable compounds for moisturization and elasticity ${ }^{210}$. Regarding to acne vulgaris, glycolic acid has shown a marked improvement in patients suffering from mild to severe conditions. The peeling effect from the fruit acid clears skin and unclogs pores, as testified by its use in concentration over $50 \%$ and a maintenance regimens with lower concentrations, $<15 \%$, to 
prevent pores occlusion. As a result, GA speeded up the clinical response acting as an adjuvant treatment, and providing more comfort to patients by diminishing the skin marks 211,212 . The advantages of glycolic acid over some of the other available peeling agents are its stability and its $\mathrm{pH}$ range under acidic conditions. Interestingly, Takenaka et al reported that 30-35 vol\% glycolic acid exhibits antibacterial activity against $C$. acnes and can decrease $C$. acnes concentrations on the checks of acne vulgaris patients in a human clinical trial ${ }^{213}$. Notably, the chemical peel used in that study contained $35 \mathrm{vol} \%$ glycolic acid at $\mathrm{pH} 1.2$, which is not suitable for over-the-counter topical use ${ }^{214}$.The maximum reported efficacy for this organic acid is at 4.40 and according to the FDA it should not go below 3.5 for over the counter commercialization ${ }^{215,216}$. The multiple assets from this acid makes it a versatile agent for acne formulations. For the treatment of atopic dermatitis, glycolic acid has proved to reduce inflammation and repair the skin barrier, leading to less outbreaks 217. The sloughing off damaged skin cells is critical for the healing process, and the gentle exfoliation that can be reached with lower concentration of glycolic acid is quiet favorable. The second benefit gained with GA exfoliation treatment is the move up of new cells to the surface. New cells are capable of retaining suitable levels of moisture, which prevents dryness and skin barrier breakage. A third benefit gained from the use of organic acids in general, is the decrease in $\mathrm{pH}$. AD patients tend to have a more alkaline skin $\mathrm{pH}$, between 0.1 and 0.9 units higher than the average healthy skin of $4.7^{9,218}$. Therefore, the adjustment of $\mathrm{pH}$ is a feasible strategy for the problem ${ }^{219,220}$. Lee and coworkers tried 5 creams made with vinegar, all with different acidity, to diminish AD lesions in mice models ${ }^{221}$. As a result, the pure reduction and maintenance of the $\mathrm{pH}$ stopped the development of eczema. Investigations with various organic acids, excluding GA, demonstrated their ability to reduce inflammation caused by Pseudomonas aeruginosa, bacteria colonizing atopic skin 222. Overall, most of the available literature points out that glycolic acid in lower concentrations substantially improves AD. Nevertheless, the mechanism of action is not fully understood and it would be advantageous to further explore the antibacterial properties of glycolic acid in order to devise more broadly useful treatment strategies that are suitable for over-the-counter application usage. 


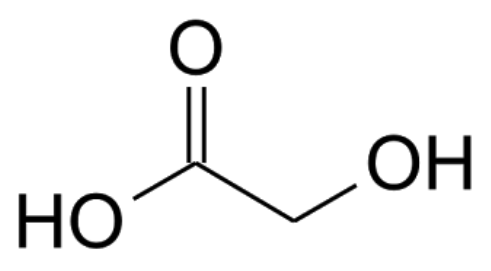

Figure 2.2. Molecular structure of Glycolic acid

\subsubsection{Experimental Characterization of Glycolic acid}

The works done on the characterization of hydroxy acids relay on the analysis in vivo of skin models or in situ trials in humans. Kessler et al compared the efficacy of glycolic and salicylic acid, alpha and beta hydroxy acids, respectively, on half of the face of 20 volunteers ${ }^{223}$.The visual assessment of the treatments showed that both peels were similarly effective. More examples of in situ trials are like the one done by Abels et al, where they studied the clinical efficacy and tolerability of an emulsion with $10 \%$ glycolic acid at a $\mathrm{pH}$ of $4^{212}$. The emulsion was the only product applied on patients with mild acne for 3 months once daily. The percentage of efficacy and tolerability was high, and the minimum adverse effects were surpassed by the outcomes. All of these works offer some knowledge of the mode of action on skin, nonetheless the mechanistic behavior of the acids against bacteria has been hindered. In this sense, only Takenaka et al have reported growth inhibitory and bactericidal assays of $C$. acnes with GA, but again, at very high concentrations and low $\mathrm{pH}$ conditions ${ }^{213}$. To provide a better mechanistic guidance, the experiments conducted in this thesis focused on the microbiological characterization with different $\mathrm{pH}^{\prime} \mathrm{s}$, funneled by the efficacy of GA and suitability for skin. The inhibitory and bactericidal concentration of glycolic acid were recorded with the MIC and MBC tests. Knowing the $\mathrm{pH}$ at which GA was more antibacterial, an imaging protocol with fluorescent counter stains was tailored to assess membrane integrity. Live/Dead viability kits are reliable techniques to distinguish live and dead microorganisms in just a matter of minutes, by using fluorescence indicators and confocal microscopy. The kit consist of two nucleic acid dyes, SYTO9 and propidium iodide (PI). SYTO9 penetrates all bacteria cells staining them in green, propidium iodide dyes in red only dead bacteria with damaged cell 
membranes. Lastly, with the confirmed concentration and $\mathrm{pH}$ at which the potency of glycolic acid is the highest, the membrane permeability of $C$. acnes bacteria was evaluated. The amount of ATP released from the bacteria cells to the medium was measured as an indicator of membrane damage. ATP is the energy source produced in the cell surface membrane of prokaryotic cells, so its detection in the media functions as a marker of bacteriolytic activity.

To recapitulate, enough findings support that glycolic acid inhibits $C$. acnes bacteria and is thus a promising agent to treat acnes vulgaris, especially since its mechanism of action could potentially offer a higher barrier to resistance development as compared to currently used antibiotics. In the case of atopic dermatitis, the use is more controversial since high concentrations worsen the disease. On that ground, $C$. acnes was prioritized in this thesis and the experiments with $S$. aureus were left pending for future works.

\subsection{Bicelles}

As stated, antimicrobial lipids, free fatty acids and monoglycerides, are an excellent therapeutic replacement for the treatment of acne and atopic dermatitis. Since they are naturally found in the skin acting as disinfectants, their application is proved to be highly effective and innocuous, with none side effects. More importantly, the mechanism of action makes it highly difficult for bacteria to develop resistance. Nonetheless, the right delivery and low water solubility of antimicrobial lipids are troublesome. Fatty acids and monoglycerides need to be formulated with another compound to be stable and available in a water-soluble presentation ${ }^{224}$, hence, an appropriate vehicle is highly desirable. Delivery vehicles are substances that carry specific drugs into contact with and through the skin ${ }^{225}$. They penetrate into deeper and target areas of skin, achieving better results than other topical products that only "sit" and interact with the upper-dead layers. In addition, they protect the active ingredient from degradation in physiological conditions and provide a longer and more sustainable effect. Delivery systems interact with the living layers of skin, offering safe and more significant benefits than the active ingredients alone 226. Bicelles were selected in this thesis for their potential in dermal applications ${ }^{227-230}$. 
Bicelles, also known as bicellar systems, are of nanometer size and achieve transdermal delivery ${ }^{227}$, however, this feature was not necessary for the project. The aim was to incorporate antimicrobial lipids, specially glycerol monolaurate, into a delivery vehicle that was small enough to penetrate into the skin appendages. The skin microbiome colonizes the outermost layer of skin and in the case of $C$. acnes deep into the hair follicles ${ }^{231}$, therefore, there was no need for penetration until the systemic system (Figure 2.3.). With reference to glycolic acid, the compound cannot be incorporated into bicelles due to its polar nature, nevertheless, its penetration can be boosted by the pretreatment with bicellar systems as widely detailed in the upcoming section. For this reason glycolic acid was set aside to include it in the final formulation. As an outline, this section explains the general characteristics, formulation and skin applications of bicelles to validate its administration with antimicrobial lipids.

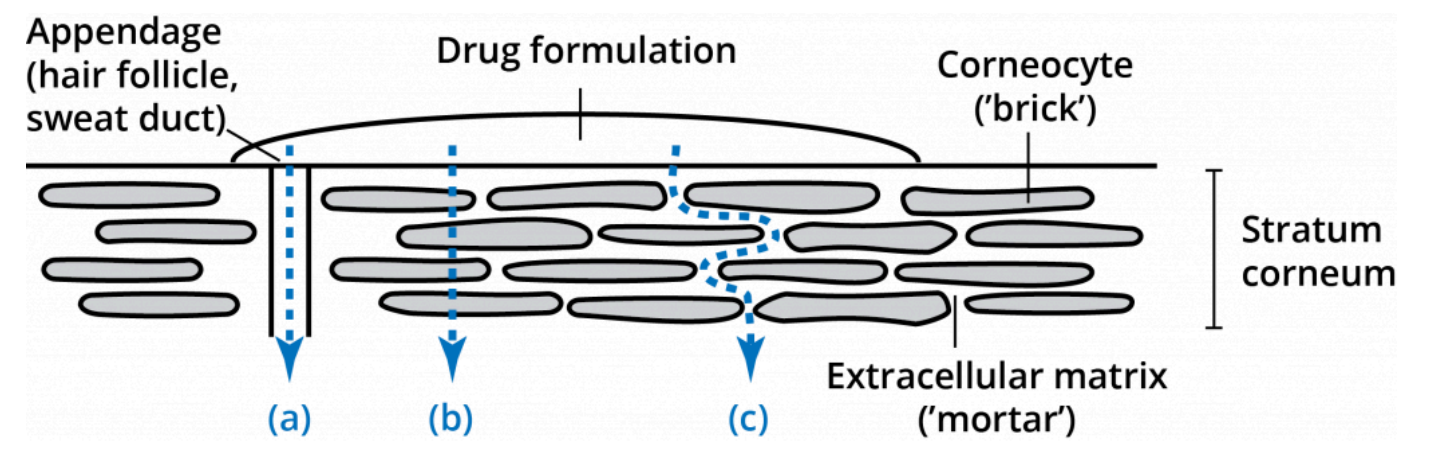

Figure 2.3. Routes of delivery systems across the epidermis. The possible pathways are: (a) through the appendages, (b) through the corneocytes (transcellular route) and (c) through the matrix layer (intercellular route) ${ }^{232}$.

\subsubsection{General description}

Bicells or bicellar systems as defined by Rodriguez et al are nanostructures formed by amphiphilic molecules, mainly long and shorth chain phospholipids, dispersed in an aqueous solution ${ }^{228,230}$. Long-chain lipids alone form lipid bilayers, while shorth-chain lipid surfactant form micellar structures; when both are mixed together they enter a liquid crystal phase, denominated lyotropic mesophase, and form bicelles ${ }^{230}$. Bicelles exhibit an 
intermediate morphology and display the most attractive properties of both systems. They form a bilayer like lipid vesicles and trap lipophilic molecules ${ }^{233}$, and similar to micelles they are non-compartmentalized, transparent and monodisperse ${ }^{229}$. The bicelle model was developed by Sanders and coworkers in 1992 for its use in high-resolution nuclear magnetic resonance (NMR) ${ }^{234}$. Bicelles spontaneously align with magnetic fields, unlike lipid vesicles and micelles that reorient in all directions ${ }^{235}$. The ideal bicelle presents a discoidal phospholipid nanostructure with a diameter of 15-50 nm and thickness of 4-6 nm 229. It contains dimyristoyl-phosphatidylcholine (DMPC) or dipalmitoylphosphatidylcholine (DPPC), which is the long-chain phospholipid and form the flat bilayer center. Dihexanoyl-phosphatidylcholine (DHPC), the short-chain lipid, is located at the edges and assembles itself as a rim to stabilize the structure (Figure 2.4.). Even thought, bicelles are recognized as disk-like assembles, bicellar systems can adopt a wide range of morphologies and sizes, depending on the type, concentration $\left(\mathrm{C}_{\mathrm{L}}\right)$ and molar ratio of long/short-chain phospholipids (q-ratio) ${ }^{229}$. Bicelles are smaller with higher concentration values $\left(\mathrm{C}_{\mathrm{L}}\right)$ and bigger with higher q-ratios. The nanostructures can be spherical micelles, discoidal micelles, tubular structures, branched structures and perforated lamellar sheets ${ }^{228}$ (Figure 2.5.). Regarding to the nanostructures size, it vary from 10 to $1000 \mathrm{~nm}^{236}$. A determining factor for the bicelle morphology is temperature, when the transition temperature $\left(T_{m}\right)$ of the long-chain phospholipid is exceed statetransitions arise and the discoidal bicelles become vesicles ${ }^{228}$. Hence, care must be taken to design bicellar systems that remain stable according to the dermal route. 


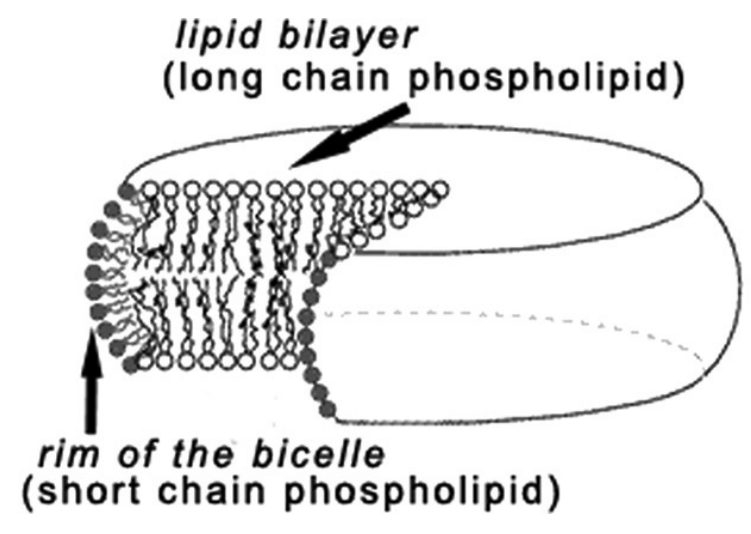

Figure 2.4. Schematic representation of the ideal bicelle model. The flat bilayer region is formed by long-chain phospholipids, normally DMPC, and the rim of short-chain phospholipids, DHPC 237.

A

B

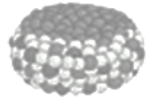

C

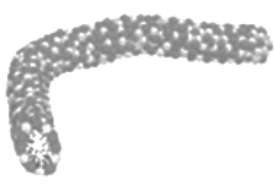

D

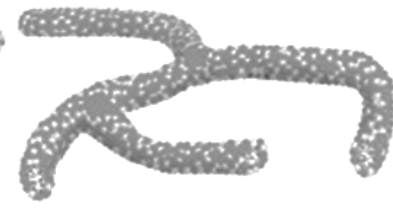

$\mathbf{E}$

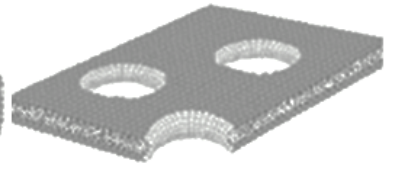

Figure 2.5. Different nanostructures adopted by bicelles. The morphology of the bicelle depends on the type, concentration and ratio of long/short -chain phospholipids. Long-chain lipids, in dark grey, tend to form bilayers and assemble in flatter areas. Short-chain lipids, in light grey, are micelle prone and reorganize in curved areas. (A) spherical micelles, (B) discoidal micelles (bicelles), (C) tubular structures, (D) branched structures and (E) perforated lamellar sheets ${ }^{228}$.

The robustness and versatility of bicelles allow their application as model membranes for the study of epidermal lipids, lipid regenerators, skin carriers, drug delivery systems, penetration enhancers and retardants ${ }^{237}$. With the intention of assembling the antimicrobial lipids with a phospholipid and release it in a target point bicelles were thought as drug carriers. They present more advantages in comparison with other colloidal carriers as liposomes and micelles 238,239 . Liposomes have a size between 200-500 nm and might penetrate into the skin appendages ${ }^{240}$, however, they are too big to penetrate into the intercellular skin spaces of $\sim 8.5 \mathrm{~nm}^{241}$. On the other hand, micelles can cause irritation because of the surfactants ${ }^{242}$. The main benefits of bicelles are their size tuning according 
the system design, the incorporation of lipophilic and amphiphilic compounds into the bilayer and their composition entirely of lipids ${ }^{229}$. Insomuch as the delivery, their notable small size permits a better penetration on skin, the major obstacle for topical products. As for the $\mathrm{pH}$, they can be adjusted within the optimal value of skin, avoiding any trouble with formulation or irritation once applied ${ }^{243,244}$. Building up on their pros, their lipophilic nature increase hydration by impeding the loss of water 227,245 . They adhere to skin and slowly release the active ingredient, leaving a lipid mantle, an effect highly desirable for occlusive products 246,247 . Depending on their lipid composition they can enhance percutaneous absorption when applied on skin before the drug application, or retard the absorption by slowly releasing the active compound ${ }^{248}$. Several publications even corroborated the greater penetration of hydrophilic compounds dissolved in aqueous solvents after pretreatment with bicelles 229,237,248. However, as any other developing technology, bicelles present some drawbacks as change in morphology and effect on skin when diluted in high-water content emulsions ${ }^{249}$. To overcome these weaknesses a second generation of bicelles aroused, denominated "bicosomes" ${ }^{249}$. These nano colloids are prepared as bicelles and next encapsulated in liposomes, which protects them from dilution and preserve them until reaching the target tissue. The leading drawbacks of bicelles seem to be fixed to facilitate their incorporation in dermal uptake. Even though, these nano assembles have remarkable features, the amount of products making use of this technology in the market is scarce because of the novelty of the carrier. Limited publications assessed the effects of them with Ceramides ${ }^{250}$, Diclofenac ${ }^{248}$, Flufenamic acid ${ }^{244}$ and $\beta$-carotene 251 . Then again, the core point is the high rate of penetration, and the fact that the release profile can be varied from fast to prolonged, depending on the inner structure.

For the purpose of this project bicelles represent the best solution for the cutaneous delivery of the formulation with antimicrobial lipids, and the later penetration of glycolic acid. These carriers guarantee the local effect, appendage penetration and time controlled delivery. Additionally, the pretreatment with bicellar systems boost the penetration of hydrophilic components $229,237,248$. 


\subsubsection{Formulation}

The formulation of bicelles follow a very straightforward and highly reproducible protocol. It begins by mixing the long-chain and short-chain lipids, according to the predetermined q-ratio (long/short-chain molarity). Subsequently, the organic solvent is removed with a rotary evaporator, or a gentle stream of nitrogen together with a complete evaporation under a vacuum desiccator. It is very important to remove any trace of organic solvent that might interfere with the process. Ultimately, the mixture of lipids or lipid-surfactant is hydrated with the right amount of buffer and submitted to several cycles of freezing and thawing in a hot bath or sonicator ${ }^{252-254}$.

\subsubsection{Morphological characterization}

The first approach to depict bicelles is the verification of their formation and quality, routinely done with Nuclear Magnetic Resonance (NMR) ${ }^{255}$. Indeed, bicelles were developed to overcome experimental problems with NMR implying the use of peptides and proteins ${ }^{256}$. Today, proteins are easily studied thanks to their crystallization with bicelles 257,258 . NMR is an spectroscopy methodology occupied to determine the content, purity and molecular structure of samples. In the case of proteins and bicelles it renders them by isotopic labeling ${ }^{259}$. NMR is the warhorse of bicellar systems study and the most common technique is ${ }^{31} \mathrm{P}$ NMR. ${ }^{31} \mathrm{P}$ states for Phosphours-31 and because of its presence in the phospholipids head groups it can probe the effects occurring in the in nanostructures and bilayers ${ }^{259}$. As a general rule, bicelles with q-ratios between 2.8 - 6.5 and lipid concentrations of $3-40 \%$ spontaneously align in parallel with the magnetic field, and NMR gives good resolution 255,260,261. Conversely, samples with q-ratios below 1 become difficult to resolve and require more sensitive techniques like Cryo-TEM, DLS, FTIR spectroscopy 262 and fluorescence spectroscopy ${ }^{263}$. For this reason, the morphological characterization of bicellar systems is consistently done using electron microscopy techniques. CryoTransmission Electron Microscopy (Cryo-TEM) is a powerful method that allows the direct observation of the "actual" bicelle structure. Even when the lipid carriers are applied on skin, Cryo-TEM aids to figure out the self-assemble of the intracellular lipids in the stratum corneum. In general, it facilitates the detailed examination of small samples and 
directly image the structure, avoiding replication procedures ${ }^{205}$. Regarding to the sample preparations, most of the bicelles protocols refer to the Hight Pressure Freezing technique (HPF $)^{229,264}$. This type of fixation does not require the sample to go through any previous treatment and rapidly freezes the specimen, as its name says under high-pressure conditions 265. Another method used to complement the observation of bicelles with Cryo-TEM is Freeze Fracture Electron Microscopy (FFEM). This technique provides a detailed image of the particle surface, simplifying in the examination of the particle topography ${ }^{266}$. It is the only EM technique that can image planar views of the internal organization of the membranes ${ }^{267}$. As an example, Rodríguez et al examined the bicelles formed with dioleylphosphatidylcholine (DOPC) and DHPC as a viable platform for dermal applications with Cryo-TEM and FFEM ${ }^{266}$. The micrographs with Cryo-TEM showed the different bicelles formed according to the q-ratio: unilamellar and multilamellar vesicles, spherical micelles and rod shaped structures, all in different sizes; but with smooth, flat and regular appearances. FFEM corroborated the nanostructures size and exposed the surface features. The vesicles displayed undulations and perforations that formed and interconnected the lamellar structures, non-evident characteristics with the first technique. Both microscopy techniques are complementary because they aid to clarify different traits of the nanostructures presented in the systems. Of note, it is highly important to utilize techniques with minimum sample pretreatment, specifically in the case of electron microscopy where very diluted specimens are frequently required. As mentioned in the general description the dilution of bicelles leads to changes in the morphology and hence, bias images. Dynamic Light Scattering (DLS) is another type of technique used to complement electron microscopy and build up on the characterization of bicelles. It provides more quantitative information regarding the size and size distribution and offers better statistics ${ }^{247}$. The particle size is determined by an incident laser passing through the sample, the intensity of the scattered light is detected and analyzed to almost immediately graph a size distribution curve ${ }^{268}$. The apparent size is resolve with the hydrodynamic radius. It is a reliable, non-invasive and fast methodology. Henceforth, at the beginning of their investigation with DOPC/DHPC bicelles, Rodríguez et al used DLS to record the size and size distribution of the particles, which led to the further analysis of the samples with EM methodologies ${ }^{247}$. 
In the matter of the characterization and monitoring of the structural changes caused by the bicelles on skin the standard techniques are: X-ray Scattering with Small Angle (SAXS), Small-Angle Neutron Scattering (SANS) and Attenuated Transmission Reflectance Fourier Transform Infrared Spectroscopy (ATR-FTIR). Considering the low amount of lipids in the stratum corneum a very sensitive instrument is required to obtain good and high quality signals. In this sense, X-ray Scattering has proven useful to gain data regarding the supra-molecular lipid organization on skin tissues without crystallization ${ }^{269}$. Scattering with Small Angle (SAXS) informs about the phase behavior and lamellarity ${ }^{228}$. It provides data of the repeat distance of the lipid lamellar phase ${ }^{266}$. The technique eases the measurement of the bicelle thickness, alluding to the short-chain lipid/surfactant rim ${ }^{270}$. The skin tissue is placed at a $90^{\circ}$ angle from the incident X-ray beam and the scattered intensity of the sample is recorded as a measurement of chemical composition and physical properties ${ }^{271}$. The main drawback is the high-energy X-ray required to gather good scattering signal from a very thin sample. Cócera and coworkers published a study regarding the collagen content in skin of young and old volunteers ${ }^{269}$. The experiments were conducted with SAXS and demonstrated how healthy and young skin tissues present a characteristic collage pattern, unlike older or damaged skin. At the bottom line, they validated the feasibility of this methodology for the diagnosis of skin conditions. Very similar to SAXS, Small-Angle Neutron Scattering (SANS) is commonly used to identify the structural phase behavior of even larger bicellar assembles ${ }^{272}$. This technique uses elastic neutron scattering to define the structure of substances in various states ${ }^{273}$. It does not require crystalline samples and the documented patterns give information about the intralamellar or interparticle spacing, typical bilayer widths, basic morphologies, and aggregate sizes or size distributions ${ }^{274}$. Multiple papers utilize SANS to characterize surfactants ${ }^{275}$, vesicles, bicelles and micelles ${ }^{276-279}$. The last technique, Attenuated Transmission Reflectance Fourier Transform Infrared Spectroscopy (ATR-FTIR) is used to study the structure of bicelles, the thermal changes and the overall organization of the lipid mantle in the epidermis ${ }^{228}$. Since the permeation depth of the IR is of $1 \mu \mathrm{m}$, it eases the study of the stratum corneum without the separation of the other skin layers ${ }^{280}$. The sample is located directly onto an infrared transparent crystal, where the light beam penetrates the skin tissue and the alkyl chains from the lipids vibrate at an specific 
frequency ${ }^{281}$. Each vibrational frequency matches a lipid order phase, for instance, highly organize lipids are in a gel phase, while less organize are in liquid-crystalline. A different publication from the bicelles forerunners, examined the tissue treated with DOPC/DHPC and concluded that the lipid arrangement on skin was in liquid-crystalline phase, they hypothesized this effect was due to the large volume of the bicelles that did not penetrate skin and remained at the top forming a protective lipid matrix ${ }^{247}$. With this assumption in mind, they concluded that the ATR-FTIR results can also help to decipher the bicelles penetration profile, making it a highly useful methodology for the overall evaluation of skin tissues.

\subsubsection{Application of Bicelles to the Skin}

The application of bicelles to skin has been tested with a few mixtures of lipids and some scatter active ingredients. Anyhow, its suitability on skin has been proven in all cases. The lipid arrangement from the bicelles prevents from the disorganization of the lipid structures in the skin barrier and the breakage of the corneocyte envelopes ${ }^{229}$. According to their composition and ratio, bicelles can penetrate deep into the lower layers of the epidermis, reinforce the lipid mantle or facilitate the penetration of other active ingredients. As described above the ideal bicelle involves the mixture of DMPC/DHPC (dimyristoylphosphatidylcholine/dihexanoyl-phosphatidylcholine). This bicelle displays a discoidal form and the smallest size, yet it undergoes phase transition and change size from $\sim 15 \mathrm{~nm}$ to $500 \mathrm{~nm}$ on contact with skin ${ }^{282}$. The transition temperature $\left(T_{m}\right)$ of DMPC is $24.1{ }^{\circ} \mathrm{C}{ }^{283}$, so at the physiological temperature of $37^{\circ} \mathrm{C}$, it changes morphology and becomes a vesicle. Due to the size transition it cannot penetrate skin and remains at the top layer. The application of DMPC/DHPC bicelles in vivo on healthy volunteers showed to increase the transepidermal water loss (TEWL) and elasticity ${ }^{237}$. The hydration levels decreased, albeit at a non-concerning degree. The TEWL, is a good indicator of barrier function integrity ${ }^{284}$, the elasticity and hydration are a measure of water content, tonicity and extensibility ${ }^{285,286}$. In conjunction these aspects and the erythema index, indicative of tolerance ${ }^{287}$, are biophysical measures for the in vivo evaluation of skincare products. To overcome the shortcoming with DMPC bicelles, the long-chain lipid was exchanged with DPPC, 
dipalmitoylphosphatidylcholine. This phospholipid is two carbons larger and its $T_{m}$ value is of $41.5^{\circ} \mathrm{C}{ }^{288}$. The size of the assemble is $15 \mathrm{~nm}$ diameter, consequently it can penetrate at intracellular level and modify profounder skin layers ${ }^{245}$. The administration on patients led to augment of TEWL and diminished hydration, although less marked than with DMPC/DHPC bicelles. With the help of SEM it was observed that DPPC/DHPC bicelles interacted with the stratum corneum and formed lipid vesicles ${ }^{245}$. As stated in the general description the bicellar systems undergo state transitions owing to the variation in temperature, molar ratio, concentration and dilution ${ }^{289}$. These transitions occur in progressive steps, starting from disk shaped at temperatures below $T_{m}$, and elongated micelles, perforated lamellar sheets and mixed multilamellar vesicles above the $T_{m}$ $243,272,290-292$.

Other lipid configurations, ratios and encapsulated active ingredients have been tested over skin models in vitro and in vivo. Rubio and coworkers tried the mixture of DMPC/DHPC and DPPC/DHPC bicelles with an anti-inflammatory agent on skin, to assess their effectiveness as drug carriers. Concurrently, they assessed the application of the bicellar systems without the drug followed by the administration of diclofenac, the antiinflammatory medication, in aqueous solvent ${ }^{248}$. As a result, the bicelles prepared with diclofenac were smaller and more compact, but their penetration rate slowed down, possibly due to the rigidity as the authors explain. The pretreatment with both bicelle mixtures lead to better diclofenac permeation, even in comparison with controls without the use of bicelles. Both systems with DMPC and DPPC conferred improvements and delays on percutaneous absorption depending on the mode of administration. This investigation opened the path for the further study of enhanced topical penetration with bicelles pre-application. Another study with anti-inflammatory drugs tried the inclusion flufenamic acid into DPPC/DHPC bicelles ${ }^{244}$. As with the experiments with diclofenac, the bicelles indicated retarded absorption in comparison to the administration of the drug alone. Non previous treatments with bicelles were conducted for this trial. Is worth to notice that the carriers gently delivered the drug, preserving the skin barrier function. On the same topic of adding active ingredients into bicelles, Barbosa-Barros et al studied the inclusion of two types of ceramides into DMPC/DHPC bicelles ${ }^{250,293}$. Ceramides are the 
most abundant lipids on the stratum corneum and regulate the lipid barrier ${ }^{294}$, enough reason to try to incorporate them into a delivery vehicle. The morphological assessment showed that bicelles formed tubular structures and liposomes (ranging from 20 to $4000 \mathrm{~nm}$ ) according to the ceramide concentration and temperature; making it highly difficult to access through any skin permeation route. With regard to bicosomes, their use in comparison with bicelles was assessed by incorporating $\beta$-carotene in their preparation ${ }^{251}$. $\beta$-carotene is an antioxidant utilized as free radical scavenger, hence, its encapsulation inside bicosomes and bicelles was expected to rise the scavenging activity. The preparation of DPPC/DHPC bicelles showed lower activity than the bicosomes made with the bicelles encased with the mixture of $80 \%$ soybean PC and $20 \%$ cholesterol. The authors attributed this effect to the greater size of bicosomes, approximately $200 \mathrm{~nm}$, which cannot penetrate skin and remain in the surface making a lipid film that protect skin from UV light and lessens the formation of free radicals. Both approaches showed to be promising applications for future dermal therapies.

On the search of new lipid compositions for bicelles Rodríguez et al tried with a phospholipid bonded to an unsaturated fatty acid in the hydrophobic group, dioleylphosphatidylcholine (DOPC) and dihexanoyl-phosphatidylcholine (DHPC) ${ }^{247}$. DOPC is well known for favoring skin penetration of cutaneous drugs ${ }^{295}$. The assessment characterized the morphology and effect on pig skin. The mixture led to the formation of unilamellar vesicles $(50-200 \mathrm{~nm})$ and tubular structures $(30-120 \mathrm{~nm})$, attributed to the low phase-transition temperature of DOPC and the mild surfactant characteristics of DHPC. DOPC has a $T_{m}$ of $-16^{\circ} \mathrm{C}{ }^{296}$, then the experimental temperatures are always above and different assembles are anticipated. The effect on pig skin was not as desired, since the vesicles remained on the surface and the tubular structures provided a better skin cell organization. Nonetheless, the outcomes led to the further study of this lipid composition on the skin of healthy volunteers; where it induced a decrease in hydration and permeability, signs of a lipid mantle being form by the vesicles in the surface ${ }^{266}$. This is of relevant interest for the development of protecting or repairing materials for skin. Another example of a modified lipid composition for the design of bicellar systems was proposed by Johansson et al, where they prepared flat bilayer disk with 1,2-distearoyl-sn-glycero-3- 
phosphocholine (DSPC), cholesterol and PEG (1,2-distearoyl-sn-glycero-3phosphoethanolamine-N-[methoxy (polyethylene glycol)-5000]) ${ }^{297,298}$. The DSPC and the cholesterol formed the laminar center and the PEG assembled as the rim on the curved areas. The disk displayed excellent long-term stability and the size could be optimized according to the polyethylene glycol ratio (Table 2.6).

Table 2.6. Lipid composition of bicelles and encapsulated ingredients for skin applications

\begin{tabular}{|c|c|}
\hline Lipid Composition & $\begin{array}{l}\text { Encapsulated active } \\
\text { ingredient }\end{array}$ \\
\hline DMPC/DHPC $^{238}$ & NA \\
\hline DPPC/DHPC ${ }^{246}$ & NA \\
\hline $\begin{array}{l}\text { DMPC/DHPC \& } \\
\text { DPPC/DHPC }^{249}\end{array}$ & $\begin{array}{l}\text { Diclofenac (anti- } \\
\text { inflammatory } \\
\text { medication) }\end{array}$ \\
\hline DPPC/DHPC ${ }^{245}$ & $\begin{array}{l}\text { flufenamic acid (anti- } \\
\text { inflammatory } \\
\text { medication) }\end{array}$ \\
\hline DMPC/DHPC ${ }^{251,294}$ & $\begin{array}{l}\text { Ceramides (lipids on } \\
\text { stratum corneum) }\end{array}$ \\
\hline $\begin{array}{l}\text { DPPC/DHPC bicelles } \\
\text { encased with soybean } \\
\text { PC and cholesterol }\end{array}$ & $\beta$-carotene (antioxidant) \\
\hline DOPC/DHPC $^{248}$ & NA \\
\hline $\begin{array}{l}\text { DSPC/cholesterol/PEG } \\
298,299\end{array}$ & NA \\
\hline
\end{tabular}

*NA: Bicelles prepared without encapsulated active ingredients.

To condense, bicelles are amphiphilic nanostructures highly suitable for the upgrade of skin uptakes. They behave as enhancers, drug retarders, lipid repair agents and protective armors for the active ingredients. Their easy preparation allows to incorporate antimicrobial lipids, like glycerol monolaurate, and facilitate the later permeation of polar compounds like glycolic acid. On these bases, bicelles with glycerol monolaurate were characterized and their effect over $S$. aureus (atopic dermatitis bacteria) was tested with microbiology proofs. 


\subsection{Outstanding Questions}

As circulating $C$. acnes and $S$. aureus strains becoming increasingly resistant to antibiotics, new therapeutic measures are needed urgently. To this end, antimicrobial lipids such as lauric acid and glycerol monolaurate appear to be promising solutions, and further understanding their mechanistic activities and integrating them together with complementary therapeutic approaches can lead to comprehensive therapeutic solutions. Motivated by this research direction, numerous outstanding questions need to be answered to translate this vision into a viable solution. Among them, which antimicrobial lipid exhibits optimal antibacterial activity against $C$. acnes under acidic, skin-relevant $\mathrm{pH}$ conditions? To date, most studies have been conducted in neutral $\mathrm{pH}$ conditions, and extending the scope of such inquires to more relevant environmental conditions is imperative as different classes of antimicrobial lipid have distinct functional groups that can be sensitive to $\mathrm{pH}$ and other environmental factors. This question is particularly relevant to consider when evaluating lauric acid and its monoglyceride derivative, glycerol monolaurate, which have the highest reported potencies among medium-chain saturated antimicrobial lipids, and identifying one of them to carry forward. Worth to mention that the assessment with $S$. aureus was not performed since multiple references proved the superiority of glycerol monolaurate over lauric acid.

In a complementary direction, it is also important to understand how the alpha hydroxy acid, glycolic acid, might exhibit antibacterial activity. While some organic acids have been reported to have antibacterial activity against gram-positive bacteria, inhibitory activity against $C$. acnes has never been evaluated. From a biological perspective, this question can first be addressed by determining if glycolic acid is capable to kill C. acnes under healthy skin conditions. The evaluation procedure is very similar to the antimicrobial lipids, with the difference that the mode of action of this acid over bacteria is unknown, and the concentrations required to kill C. acnes might be over the desired ranges. Depending on the results the alpha hydroxy acid would be set aside, or kept as an adjuvant therapy with the antimicrobial lipid. 
The last set of questions encompass the delivery carrier, which as described above will be attempted with bicelles. Is the effect of the antimicrobial lipid against bacteria enhanced with a delivery carrier? How big does the bicelles becomes after the incorporation of the antimicrobial lipid? Would it remain on the top of skin as a drug reservoir, slowly releasing the compound? Can the pretreatment with antimicrobial lipids assembled as bicelles boost the penetration of glycolic acid? All of these questions must be answered to gauge if the antimicrobial lipids are worth to be assemble as bicelles or directly applied in topical form. 


\section{References}

1 Perman, M. J. \& Lucky, A. W. in Severe Skin Diseases in Children 37-51 (Springer, 2014).

2 Global Market Study on Acne Treatment: Inflammatory Acne Segment Anticipated to Account for 195 BPS Gain in Market Revenue Share During 2017 - 2025. Report No. PMRREP5279, 184 (Persistence Market Research, 2017).

3 Bickers, D. R. et al. The burden of skin diseases: 2004: a joint project of the American Academy of Dermatology Association and the Society for Investigative Dermatology. Journal of the American Academy of Dermatology 55, 490-500 (2006).

4 Bhate, K. \& Williams, H. Epidemiology of acne vulgaris. British Journal of Dermatology 168, 474-485 (2013).

5 Achermann, Y., Goldstein, E. J., Coenye, T. \& Shirtliff, M. E. Propionibacterium acnes: from commensal to opportunistic biofilm-associated implant pathogen. Clinical microbiology reviews 27, 419-440 (2014).

6 Fitz-Gibbon, S. et al. Propionibacterium acnes strain populations in the human skin microbiome associated with acne. Journal of Investigative Dermatology 133, 21522160 (2013).

7 Fitzpatrick, J. E. \& Morelli, J. G. Dermatology secrets plus. [electronic resource]. (Philadelphia, PA : Elsevier/Mosby, c2011.

4th ed., 2011).

8 Walton, S., Wyatt, E. H. \& Cunliffe, W. J. Genetic control of sebum excretion and acne-a twin study. British Journal of Dermatology 118, 393-396, doi:10.1111/j.1365-2133.1988.tb02433.x (1988).

9 Lambers, H., Piessens, S., Bloem, A., Pronk, H. \& Finkel, P. Natural skin surface $\mathrm{pH}$ is on average below 5, which is beneficial for its resident flora. International journal of cosmetic science 28, 359-370 (2006).

10 Dreno, B. et al. Development and evaluation of a global acne severity scale (GEA scale) suitable for France and Europe. Journal of the European Academy of Dermatology and Venereology 25, 43-48 (2011). 
11 Arowojolu, A. O. Combined oral contraceptive pills for treatment of acne. Cochrane Database of Systematic Reviews (2012).

12 Nguyen, R. \& Su, J. Treatment of acne vulgaris. Paediatrics and Child Health 21, 119-125, doi:http://dx.doi.org/10.1016/j.paed.2010.09.012 (2011).

13 Layton, A., Dreno, B., Gollnick, H. \& Zouboulis, C. A review of the European Directive for prescribing systemic isotretinoin for acne vulgaris. Journal of the European Academy of Dermatology and Venereology 20, 773-776 (2006).

14 Kober, M.-M., Bowe, W. P. \& Shalita, A. R. in Acneiform Eruptions in Dermatology 19-25 (Springer, 2014).

15 Burchacka, E. et al. New effective azelaic acid liposomal gel formulation of enhanced pharmaceutical bioavailability. Biomedicine \& Pharmacotherapy 83, 771-775, doi:http://dx.doi.org/10.1016/j.biopha.2016.07.014 (2016).

16 Okamoto, K. et al. In vitro antimicrobial activity of benzoyl peroxide against Propionibacterium acnes assessed by a novel susceptibility testing method. Journal of Infection and Chemotherapy 22, 426-429, doi:http://dx.doi.org/10.1016/j.jiac.2015.12.010 (2016).

17 Farrah, G. \& Tan, E. The use of oral antibiotics in treating acne vulgaris: a new approach. Dermatologic Therapy 29, 377-384, doi:10.1111/dth.12370 (2016).

18 Wozel, G. \& Blasum, C. Dapsone in dermatology and beyond. Archives of Dermatological Research 306, 103-124, doi:10.1007/s00403-013-1409-7 (2014).

19 Hoover, W. D., Davis, S. A., Fleischer, A. B. \& Feldman, S. R. Topical antibiotic monotherapy prescribing practices in acne vulgaris. Journal of Dermatological Treatment 25, 97-99, doi:10.3109/09546634.2013.852297 (2014).

20 Weidinger, S., Beck, L. A., Bieber, T., Kabashima, K. \& Irvine, A. D. Atopic dermatitis. Nature Reviews Disease Primers 4, 1, doi:10.1038/s41572-018-0001-z (2018).

21 Nutten, S. Atopic dermatitis: global epidemiology and risk factors. Annals of nutrition and metabolism 66, 8-16 (2015).

22 Eichenfield, L. F. et al. Guidelines of care for the management of atopic dermatitis: section 1. Diagnosis and assessment of atopic dermatitis. Journal of the American Academy of Dermatology 70, 338-351, doi:10.1016/j.jaad.2013.10.010 (2014). 
23 Hanifin, J. M., Reed, M. L. \& Group, I. W. A population-based survey of eczema prevalence in the United States. Dermatitis 18, 82-91 (2007).

24 Ellis, C. N., Mancini, A. J., Paller, A. S., Simpson, E. L. \& Eichenfield, L. F. in Seminars in cutaneous Medicine and Surgery. S18-S22 (WB Saunders).

25 Margolis, J. S., Abuabara, K., Bilker, W., Hoffstad, O. \& Margolis, D. J. Persistence of mild to moderate atopic dermatitis. JAMA dermatology 150, 593-600 (2014).

26 Kim, J. P., Chao, L. X., Simpson, E. L. \& Silverberg, J. I. Persistence of atopic dermatitis (AD): a systematic review and meta-analysis. Journal of the American Academy of Dermatology 75, 681-687. e611 (2016).

27 Avena-Woods, C. Overview of atopic dermatitis. The American journal of managed care 23, S115-S123 (2017).

28 Filanovsky, M. G. et al. The financial and emotional impact of atopic dermatitis on children and their families. The Journal of pediatrics 169, 284-290. e285 (2016).

29 Center, Q. I., Consult, D. C. \& Apps, A. Skin conditions by the numbers.

30 Ellinghaus, D. et al. High-density genotyping study identifies four new susceptibility loci for atopic dermatitis. Nature genetics 45, 808 (2013).

31 Paternoster, L. et al. Meta-analysis of genome-wide association studies identifies three new risk loci for atopic dermatitis. Nature genetics 44, 187-192 (2012).

32 Hirota, T. et al. Genome-wide association study identifies eight new susceptibility loci for atopic dermatitis in the Japanese population. Nature genetics 44, 1222 (2012).

33 Paternoster, L. et al. Multi-ancestry genome-wide association study of 21,000 cases and 95,000 controls identifies new risk loci for atopic dermatitis. Nature genetics 47, 1449 (2015).

34 Bierber, T. Mechanisms of disease: atopic dermatitis. N Engl J Med 358, 358-1483 (2008).

35 Sandilands, A., Sutherland, C., Irvine, A. D. \& McLean, W. I. Filaggrin in the frontline: role in skin barrier function and disease. Journal of cell science 122, 1285-1294 (2009). 
36 Baurecht, H. et al. Toward a major risk factor for atopic eczema: meta-analysis of filaggrin polymorphism data. Journal of Allergy and Clinical Immunology 120, 1406-1412 (2007).

37 Wen, H. J. et al. Predicting risk for early infantile atopic dermatitis by hereditary and environmental factors. British Journal of Dermatology 161, 1166-1172 (2009).

38 Küster, W., Petersen, M., Christophers, E., Goos, M. \& Sterry, W. A family study of atopic dermatitis. Archives of dermatological research 282, 98-102 (1990).

39 RUIZ, R. G. G., KEMENY, D. M. \& PRICE, J. F. Higher risk of infantile atopic dermatitis from maternal atopy than from paternal atopy. Clinical \& Experimental Allergy 22, 762-766, doi:10.1111/j.1365-2222.1992.tb02816.x (1992).

40 Weidinger, S. et al. A genome-wide association study of atopic dermatitis identifies loci with overlapping effects on asthma and psoriasis. Human molecular genetics 22, 4841-4856 (2013).

41 Jungersted, J. et al. Stratum corneum lipids, skin barrier function and filaggrin mutations in patients with atopic eczema. Allergy 65, 911-918 (2010).

42 Flohr, C. et al. Filaggrin loss-of-function mutations are associated with early-onset eczema, eczema severity and transepidermal water loss at 3 months of age. British Journal of Dermatology 163, 1333-1336 (2010).

43 Danby, S. G. et al. The effect of water hardness on surfactant deposition after washing and subsequent skin irritation in atopic dermatitis patients and healthy control subjects. Journal of Investigative Dermatology 138, 68-77 (2018).

44 Gao, P.-S. et al. Filaggrin mutations that confer risk of atopic dermatitis confer greater risk for eczema herpeticum. Journal of allergy and clinical immunology 124, 507-513. e507 (2009).

45 Miajlovic, H., Fallon, P. G., Irvine, A. D. \& Foster, T. J. Effect of filaggrin breakdown products on growth of and protein expression by Staphylococcus aureus. Journal of Allergy and Clinical Immunology 126, 1184-1190. e1183 (2010).

46 Pellerin, L. et al. Defects of filaggrin-like proteins in both lesional and nonlesional atopic skin. Journal of allergy and clinical immunology 131, 1094-1102 (2013).

47 Rawlings, A. V. \& Voegeli, R. Stratum corneum proteases and dry skin conditions. Cell and tissue research 351, 217-235 (2013). 
48 Janssens, M. et al. Increase in short-chain ceramides correlates with an altered lipid organization and decreased barrier function in atopic eczema patients. Journal of lipid research 53, 2755-2766 (2012).

49 De Benedetto, A. et al. Tight junction defects in patients with atopic dermatitis. Journal of Allergy and Clinical Immunology 127, 773-786. e777 (2011).

50 Kong, H. H. et al. Temporal shifts in the skin microbiome associated with disease flares and treatment in children with atopic dermatitis. Genome research 22, 850859 (2012).

51 Byrd, A. L. et al. Staphylococcus aureus and Staphylococcus epidermidis strain diversity underlying pediatric atopic dermatitis. Science translational medicine $\mathbf{9}$, eaal4651 (2017).

52 Huang, J. T., Abrams, M., Tlougan, B., Rademaker, A. \& Paller, A. S. Treatment of Staphylococcus aureus colonization in atopic dermatitis decreases disease severity. Pediatrics 123, e808-e814 (2009).

53 LEYDEN, J. J., MARPLES, R. R. \& KLIGMAN, A. M. Staphylococcus aureus in the lesions of atopic dermatitis. British Journal of Dermatology 90, 525-525 (1974).

54 Hanifin, J. M. \& Rogge, J. L. Staphylococcal infections in patients with atopic dermatitis. Archives of dermatology 113, 1383-1386 (1977).

55 Birnie, A., Bath-Hextall, F., Ravenscroft, J. \& Williams, H. Interventions to reduce Staphylococcus aureus in the management of atopic eczema. Cochrane database of systematic reviews (Online) 3, CD003871, doi:10.1002/14651858.CD003871.pub2 (2008).

56 Berke, R., Singh, A. \& Guralnick, M. Atopic dermatitis: an overview. American family physician 86, 35-42 (2012).

57 Chopra, R. et al. Severity strata for Eczema Area and Severity Index (EASI), modified EASI, Scoring Atopic Dermatitis (SCORAD), objective SCORAD, Atopic Dermatitis Severity Index and body surface area in adolescents and adults with atopic dermatitis. British Journal of Dermatology 177, 1316-1321 (2017).

58 Leshem, Y., Hajar, T., Hanifin, J. \& Simpson, E. What the Eczema Area and Severity Index score tells us about the severity of atopic dermatitis: an interpretability study. British Journal of Dermatology 172, 1353-1357 (2015). 
59 Schmitt, J., Langan, S., Williams, H. C. \& Network, E. D.-E. What are the best outcome measurements for atopic eczema? A systematic review. Journal of Allergy and Clinical Immunology 120, 1389-1398 (2007).

60 Kim, B. S. Atopic dermatitis, $<$ https://emedicine.medscape.com/article/1049085overview $>$ (2016).

61 Hahn, E. L. \& Bacharier, L. B. The atopic march: the pattern of allergic disease development in childhood. Immunology and Allergy Clinics 25, 231-246 (2005).

62 Burgess, J. A. et al. Childhood eczema and asthma incidence and persistence: a cohort study from childhood to middle age. Journal of Allergy and Clinical Immunology 122, 280-285 (2008).

63 Tan, R. A. \& Corren, J. The relationship of rhinitis and asthma, sinusitis, food allergy, and eczema. Immunology and Allergy Clinics 31, 481-491 (2011).

64 van der Hulst, A. E., Klip, H. \& Brand, P. L. Risk of developing asthma in young children with atopic eczema: a systematic review. Journal of Allergy and Clinical Immunology 120, 565-569 (2007).

65 Spergel, J. M. Epidemiology of atopic dermatitis and atopic march in children. Immunology and Allergy Clinics 30, 269-280 (2010).

66 Strom, M., Fishbein, A., Paller, A. \& Silverberg, J. I. Association between atopic dermatitis and attention deficit hyperactivity disorder in US children and adults. British Journal of Dermatology 175, 920-929 (2016).

67 Strom, M. A. \& Silverberg, J. I. Eczema is associated with childhood speech disorder: A retrospective analysis from the National Survey of Children's Health and the National Health Interview Survey. The Journal of pediatrics 168, 185-192. e184 (2016).

68 Drucker, A. M. in Allergy \& Asthma Proceedings.

69 Linnet, J. \& Jemec, G. An assessment of anxiety and dermatology life quality in patients with atopic dermatitis. The British journal of dermatology 140, 268-272 (1999).

70 Siegfried, E. C. \& Hebert, A. A. Diagnosis of atopic dermatitis: mimics, overlaps, and complications. Journal of clinical medicine 4, 884-917 (2015). 
71 Hanifin, J. M. Diagnostic features of atopic dermatitis. Acta Derm Venereol (Suppl) 92, 44-47 (1980).

72 Eichenfield, L. F., Hanifin, J. M., Luger, T. A., Stevens, S. R. \& Pride, H. B. Consensus conference on pediatric atopic dermatitis. Journal of the American Academy of Dermatology 49, 1088-1095 (2003).

73 Cork, M. et al. Comparison of parent knowledge, therapy utilization and severity of atopic eczema before and after explanation and demonstration of topical therapies by a specialist dermatology nurse. British Journal of Dermatology 149, 582-589 (2003).

74 Brunner, P. M. et al. A mild topical steroid leads to progressive anti-inflammatory effects in the skin of patients with moderate-to-severe atopic dermatitis. Journal of Allergy and Clinical Immunology 138, 169-178 (2016).

75 Nakahara, T., Morimoto, H., Murakami, N. \& Furue, M. Mechanistic insights into topical tacrolimus for the treatment of atopic dermatitis. Pediatric Allergy and Immunology 29, 233-238 (2018).

76 Jensen, J.-M. et al. Effects of pimecrolimus compared with triamcinolone acetonide cream on skin barrier structure in atopic dermatitis: A randomized, double-blind, right-left arm trial. Acta dermato-venereologica 93, 515-519 (2013).

77 Zebda, R. \& Paller, A. S. Phosphodiesterase 4 inhibitors. Journal of the American Academy of Dermatology 78, S43-S52 (2018).

78 Eichenfield, L. F. et al. Long-term safety of crisaborole ointment $2 \%$ in children and adults with mild to moderate atopic dermatitis. Journal of the American Academy of Dermatology 77, 641-649. e645 (2017).

79 Garritsen, F., Brouwer, M., Limpens, J. \& Spuls, P. I. Photo (chemo) therapy in the management of atopic dermatitis: an updated systematic review with implications for practice and research. British Journal of Dermatology 170, 501-513 (2014).

80 Rodenbeck, D. L., Silverberg, J. I. \& Silverberg, N. B. Phototherapy for atopic dermatitis. Clinics in dermatology 34, 607-613 (2016).

81 Belloni, A. F. \& Neri, L. Antibiotic therapy in the management of atopic dermatitis. Giornale italiano di dermatologia e venereologia: organo ufficiale, Societa italiana di dermatologia e sifilografia 150, 321-325 (2015). 
82 Flohr, C. \& Irvine, A. D. Systemic therapies for severe atopic dermatitis in children and adults. Journal of Allergy and Clinical Immunology 132, 774 (2013).

83 Schmitt, J., Schmitt, N. \& Meurer, M. Cyclosporin in the treatment of patients with atopic eczema-a systematic review and meta-analysis. Journal of the European Academy of Dermatology and Venereology 21, 606-619 (2007).

84 Goujon, C. et al. Methotrexate versus cyclosporine in adults with moderate-tosevere atopic dermatitis: a phase III randomized noninferiority trial. The Journal of Allergy and Clinical Immunology: In Practice 6, 562-569. e563 (2018).

85 Fuggle, N. R. et al. The adverse effect profile of oral azathioprine in pediatric atopic dermatitis, and recommendations for monitoring. Journal of the American Academy of Dermatology 72, 108-114 (2015).

86 Haeck, I. M. et al. Enteric-coated mycophenolate sodium versus cyclosporin A as long-term treatment in adult patients with severe atopic dermatitis: a randomized controlled trial. Journal of the American Academy of Dermatology 64, 1074-1084 (2011).

87 Mohan, G. C. \& Lio, P. A. Comparison of dermatology and allergy guidelines for atopic dermatitis management. JAMA dermatology 151, 1009-1013 (2015).

88 Beck, L. A. et al. Dupilumab treatment in adults with moderate-to-severe atopic dermatitis. New England Journal of Medicine 371, 130-139 (2014).

89 Blauvelt, A. et al. Long-term management of moderate-to-severe atopic dermatitis with dupilumab and concomitant topical corticosteroids (LIBERTY AD CHRONOS): a 1-year, randomised, double-blinded, placebo-controlled, phase 3 trial. The Lancet 389, 2287-2303 (2017).

90 Finch, J., Munhutu, M. \& Whitaker-Worth, D. L. Atopic dermatitis and nutrition. Clinics in dermatology 28, 605-614 (2010).

91 Rosenfeldt, V., Benfeldt, E., Valerius, N. H., Pærregaard, A. \& Michaelsen, K. F. Effect of probiotics on gastrointestinal symptoms and small intestinal permeability in children with atopic dermatitis. The Journal of pediatrics 145, 612-616 (2004).

92 Majamaa, H. \& Isolauri, E. Evaluation of the gut mucosal barrier: evidence for increased antigen transfer in children with atopic eczema. Journal of allergy and clinical immunology 97, 985-990 (1996). 
93 Foolad, N., Brezinski, E. A., Chase, E. P. \& Armstrong, A. W. Effect of nutrient supplementation on atopic dermatitis in children: a systematic review of probiotics, prebiotics, formula, and fatty acids. JAMA dermatology 149, 350-355 (2013).

94 Viljanen, M. et al. Probiotics in the treatment of atopic eczema/dermatitis syndrome in infants: a double-blind placebo-controlled trial. Allergy 60, 494-500 (2005).

95 Gueniche, A. et al. Lactobacillus paracasei CNCM I-2116 (ST11) inhibits substance P-induced skin inflammation and accelerates skin barrier function recovery in vitro. European Journal of Dermatology 20, 731-737 (2010).

96 Drago, L., Toscano, M., De Vecchi, E., Piconi, S. \& Iemoli, E. Changing of fecal flora and clinical effect of L. salivarius LS01 in adults with atopic dermatitis. Journal of clinical gastroenterology 46, S56-S63 (2012).

97 Mendoza, N., Hernandez, P. O., Tyring, S. K., Haitz, K. A. \& Motta, A. Antimicrobial susceptibility of Propionibacterium acnes isolates from acne patients in Colombia. International Journal of Dermatology 52, 688-692, doi:10.1111/j.1365-4632.2011.05403.x (2013).

98 González, R. et al. Report: In vitro antimicrobial susceptibility of Propionibacterium acnes isolated from acne patients in northern Mexico. International Journal of Dermatology 49, 1003-1007, doi:10.1111/j.13654632.2010.04506.x (2010).

99 Moon, S. H. et al. Antibiotic resistance of microbial strains isolated from Korean acne patients. The Journal of dermatology 39, 833-837 (2012).

100 Ross, J. et al. Antibiotic-resistant acne: lessons from Europe. British journal of Dermatology 148, 467-478 (2003).

101 Abdel Fattah, N. \& Darwish, Y. In vitro antibiotic susceptibility patterns of Propionibacterium acnes isolated from acne patients: an Egyptian university hospital-based study. Journal of the European Academy of Dermatology and Venereology 27, 1546-1551 (2013).

102 Dessinioti, C. \& Katsambas, A. Propionibacterium acnes and antimicrobial resistance in acne. Clinics in Dermatology (2016).

103 Kabara, J. J. Antimicrobial agents derived from fatty acids. Journal of the American Oil Chemists’ Society 61, 397-403 (1984). 
104 Smith, S. Mechanism of chain length determination in biosynthesis of milk fatty acids. Journal of Dairy Science 63, 337-352 (1980).

105 Cera, K., Mahan, D. \& Reinhart, G. Apparent fat digestibilities and performance responses of postweaning swine fed diets supplemented with coconut oil, corn oil or tallow. Journal of Animal Science 67, 2040-2047 (1989).

106 Thormar, H. Lipids and essential oils as antimicrobial agents. (John Wiley \& Sons, 2010).

107 Blevins, S. M. \& Bronze, M. S. Robert Koch and the 'golden age' of bacteriology. International Journal of Infectious Diseases 14, e744-e751, doi:https://doi.org/10.1016/j.ijid.2009.12.003 (2010).

108 Thormar, H. Antibacterial effects of lipids: historical review (1881 to 1960). Lipids and Essential Oils as Antimicrobial Agents, 25-45 (2011).

109 Burtenshaw, J. The mechanism of self-disinfection of the human skin and its appendages. Epidemiology \& Infection 42, 184-210 (1942).

110 Aminov, R. I. A brief history of the antibiotic era: lessons learned and challenges for the future. Frontiers in microbiology 1, 134 (2010).

111 Alanis, A. J. Resistance to antibiotics: are we in the post-antibiotic era? Archives of medical research 36, 697-705 (2005).

112 Kabara, J. J., Swieczkowski, D. M., Conley, A. J. \& Truant, J. P. Fatty acids and derivatives as antimicrobial agents. Antimicrobial agents and chemotherapy 2, 2328 (1972).

113 Kodicek, E. \& Worden, A. The effect of unsaturated fatty acids on Lactobacillus helveticus and other Gram-positive micro-organisms. Biochemical Journal 39, 78 (1945).

114 Galbraith, H., Miller, T., Paton, A. \& Thompson, J. Antibacterial activity of long chain fatty acids and the reversal with calcium, magnesium, ergocalciferol and cholesterol. Journal of applied Bacteriology 34, 803-813 (1971).

115 Desbois, A. P. \& Smith, V. J. Antibacterial free fatty acids: activities, mechanisms of action and biotechnological potential. Applied microbiology and biotechnology 85, 1629-1642 (2010). 
116 Kakisawa, H. et al. An allelopathic fatty acid from the brown alga Cladosiphon okamuranus. Phytochemistry 27, 731-735 (1988).

117 Kumaratilake, L., Robinson, B., Ferrante, A. \& Poulos, A. Antimalarial properties of n-3 and n-6 polyunsaturated fatty acids: in vitro effects on Plasmodium falciparum and in vivo effects on P. berghei. The Journal of clinical investigation 89, 961-967 (1992).

118 Fu, X., Feng, F. \& Huang, B. Physicochemical characterization and evaluation of a microemulsion system for antimicrobial activity of glycerol monolaurate. International journal of pharmaceutics 321, 171-175 (2006).

119 P Desbois, A. Potential applications of antimicrobial fatty acids in medicine, agriculture and other industries. Recent patents on anti-infective drug discovery 7 , 111-122 (2012).

120 Jackman, J. A., Boyd, R. D. \& Elrod, C. C. Medium-chain fatty acids and monoglycerides as feed additives for pig production: towards gut health improvement and feed pathogen mitigation. Journal of Animal Science and Biotechnology 11, 1-15 (2020).

121 Davies, J. \& Davies, D. Origins and evolution of antibiotic resistance. Microbiol. Mol. Biol. Rev. 74, 417-433 (2010).

122 Yoon, B. K., Jackman, J. A., Valle-González, E. R. \& Cho, N.-J. Antibacterial free fatty acids and monoglycerides: biological activities, experimental testing, and therapeutic applications. International journal of molecular sciences 19, 1114 (2018).

123 Jacobsen, C. in Encyclopedia of Food Chemistry (eds Laurence Melton, Fereidoon Shahidi, \& Peter Varelis) 261-269 (Academic Press, 2019).

124 Thormar, H. \& Hilmarsson, H. The role of microbicidal lipids in host defense against pathogens and their potential as therapeutic agents. Chemistry and Physics of Lipids 150, 1-11 (2007).

125 Rohrer, L., Winterhalter, K., Eckert, J. \& Köhler, P. Killing of Giardia lamblia by human milk is mediated by unsaturated fatty acids. Antimicrobial agents and chemotherapy 30, 254-257 (1986). 
126 Hilmarsson, H., Larusson, L. \& Thormar, H. Virucidal effect of lipids on visna virus, a lentivirus related to HIV. Archives of virology 151, 1217-1224 (2006).

127 Wang, L.-L. \& Johnson, E. A. Inhibition of Listeria monocytogenes by fatty acids and monoglycerides. Applied and environmental microbiology 58, 624-629 (1992).

128 Petschow, B. W., Batema, R. P. \& Ford, L. L. Susceptibility of Helicobacter pylori to bactericidal properties of medium-chain monoglycerides and free fatty acids. Antimicrobial agents and chemotherapy 40, 302-306 (1996).

129 Knapp, H. R. \& Melly, M. A. Bactericidal effects of polyunsaturated fatty acids. Journal of Infectious Diseases 154, 84-94 (1986).

130 Feldlaufer, M., Knox, D., Lusby, W. \& Shimanuki, H. Antimicrobial activity of fatty acids against Bacillus larvae, the causative agent of American foulbrood disease. Apidologie 24, 95-99 (1993).

131 Kanetsuna, F. Bactericidal effect of fatty acids on mycobacteria, with particular reference to the suggested mechanism of intracellular killing. Microbiology and immunology 29, 127-141 (1985).

132 Yang, D. et al. The antimicrobial activity of liposomal lauric acids against Propionibacterium acnes. Biomaterials 30, 6035-6040 (2009).

133 Nakatsuji, T. et al. Antimicrobial property of lauric acid against Propionibacterium acnes: its therapeutic potential for inflammatory acne vulgaris. Journal of investigative dermatology 129, 2480-2488 (2009).

134 Pornpattananangkul, D. et al. In vivo treatment of Propionibacterium acnes infection with liposomal lauric acids. Advanced healthcare materials 2, 1322-1328 (2013).

135 Hong Lioe Ko, S., Heczko, P. B. \& Pulverer, G. Differential susceptibility of Propionibacterium acnes, P. granulosum and P. avidum to free fatty acids. Journal of Investigative Dermatology 71 (1978).

136 Yoon, B. K., Jackman, J. A., Kim, M. C. \& Cho, N.-J. Spectrum of membrane morphological responses to antibacterial fatty acids and related surfactants. Langmuir 31, 10223-10232 (2015). 
137 Tangwatcharin, P. \& Khopaibool, P. Activity of virgin coconut oil, lauric acid or monolaurin in combination with lactic acid against Staphylococcus aureus. Southeast Asian Journal of Tropical Medicine \& Public Health 43, 969-985 (2012).

138 Huang, W.-C. et al. Anti-bacterial and anti-inflammatory properties of capric acid against Propionibacterium acnes: a comparative study with lauric acid. Journal of dermatological science 73, 232-240 (2014).

139 Kabara, J. J. Structure-function relationships of surfactants as antimicrobial agents. J Soc Cosmet Chem 29, 733-741 (1978).

140 Kawakami, L. M. et al. Understanding how sterols regulate membrane remodeling in supported lipid bilayers. Langmuir 33, 14756-14765 (2017).

141 Yoon, B. K., Jackman, J. A., Park, S., Mokrzecka, N. \& Cho, N.-J. Characterizing the Membrane-Disruptive Behavior of Dodecylglycerol Using Supported Lipid Bilayers. Langmuir 35, 3568-3575 (2019).

142 Galbraith, H. \& Miller, T. Effect of long chain fatty acids on bacterial respiration and amino acid uptake. Journal of Applied Bacteriology 36, 659-675 (1973).

143 Sheu, C. W. \& Freese, E. Effects of fatty acids on growth and envelope proteins of Bacillus subtilis. Journal of bacteriology 111, 516-524 (1972).

144 Schlievert, P. M. \& Peterson, M. L. Glycerol monolaurate antibacterial activity in broth and biofilm cultures. PloS one 7, e40350 (2012).

145 Greenway, D. \& Dyke, K. Mechanism of the inhibitory action of linoleic acid on the growth of Staphylococcus aureus. Microbiology 115, 233-245 (1979).

146 Kurihara, H., Goto, Y., Aida, M., Hosokawa, M. \& Takahashi, K. Antibacterial activity against cariogenic bacteria and inhibition of insoluble glucan production by free fatty acids obtained from dried Gloiopeltis furcata. Fisheries science $\mathbf{6 5}$, 129-132 (1999).

147 Won, S.-R. et al. Oleic acid: an efficient inhibitor of glucosyltransferase. FEBS letters 581, 4999-5002 (2007).

148 Levison, M. E. Pharmacodynamics of antimicrobial drugs. Infectious Disease Clinics 18, 451-465 (2004).

149 Bergsson, G., Arnfinnsson, J., SteingrÍmsson, Ó. \& Thormar, H. Killing of Grampositive cocci by fatty acids and monoglycerides. APMIS 109, 670-678 (2001). 
150 Bergsson, G., Arnfinnsson, J., Steingrímsson, Ó. \& Thormar, H. In vitro killing of Candida albicans by fatty acids and monoglycerides. Antimicrobial agents and chemotherapy 45, 3209-3212 (2001).

151 Hyldgaard, M., Sutherland, D. S., Sundh, M., Mygind, T. \& Meyer, R. L. Antimicrobial mechanism of monocaprylate. Applied and Environmental Microbiology 78, 2957-2965 (2012).

152 Lonchin, S., Luisi, P. L., Walde, P. \& Robinson, B. H. A matrix effect in mixed phospholipid/fatty acid vesicle formation. The Journal of Physical Chemistry B 103, 10910-10916 (1999).

153 Rogerson, M. L., Robinson, B. H., Bucak, S. \& Walde, P. Kinetic studies of the interaction of fatty acids with phosphatidylcholine vesicles (liposomes). Colloids and Surfaces B: Biointerfaces 48, 24-34 (2006).

154 Peterlin, P., Arrigler, V., Kogej, K., Svetina, S. \& Walde, P. Growth and shape transformations of giant phospholipid vesicles upon interaction with an aqueous oleic acid suspension. Chemistry and physics of lipids 159, 67-76 (2009).

155 Mally, M., Peterlin, P. \& Svetina, S. a. Partitioning of oleic acid into phosphatidylcholine membranes is amplified by strain. The Journal of Physical Chemistry B 117, 12086-12094 (2013).

156 Yoon, B. K., Jackman, J. A., Kim, M. C., Sut, T. N. \& Cho, N.-J. Correlating Membrane Morphological Responses with Micellar Aggregation Behavior of Capric Acid and Monocaprin. Langmuir 33, 2750-2759 (2017).

157 Mechler, A. et al. Specific and selective peptide-membrane interactions revealed using quartz crystal microbalance. Biophysical Journal 93, 3907-3916 (2007).

158 Thid, D., Benkoski, J. J., Svedhem, S., Kasemo, B. \& Gold, J. DHA-induced changes of supported lipid membrane morphology. Langmuir 23, 5878-5881 (2007).

159 Flynn, K. R., Martin, L. L., Ackland, M. L. \& Torriero, A. A. Real-Time Quartz Crystal Microbalance Monitoring of Free Docosahexaenoic Acid Interactions with Supported Lipid Bilayers. Langmuir 32, 11717-11727 (2016).

160 Heerklotz, H. Interactions of surfactants with lipid membranes. Quarterly reviews of biophysics 41, 205-264 (2008). 
161 Staykova, M., Arroyo, M., Rahimi, M. \& Stone, H. A. Confined bilayers passively regulate shape and stress. Physical Review Letters 110, 028101 (2013).

162 Kawakami, L. M. et al. Understanding How Sterols Regulate Membrane Remodeling in Supported Lipid Bilayers. Langmuir (2017).

163 Chen, Y. E. \& Tsao, H. The skin microbiome: current perspectives and future challenges. Journal of the American Academy of Dermatology 69, 143-155. e143 (2013).

164 Flanagan, J. L. et al. Glycerol Monolaurate Inhibits Lipase Production by Clinical Ocular Isolates Without Affecting Bacterial Cell ViabilityGlycerol Monolaurate Inhibits Bacterial Lipase Production. Investigative ophthalmology \& visual science 57, 544-550 (2016).

165 Mueller, E. A. \& Schlievert, P. M. Non-aqueous glycerol monolaurate gel exhibits antibacterial and anti-biofilm activity against Gram-positive and Gram-negative pathogens. PloS one 10, e0120280 (2015).

166 Sun, C. Q., O'Connor, C. J. \& Roberton, A. M. Antibacterial actions of fatty acids and monoglycerides against Helicobacter pylori. FEMS Immunology \& Medical Microbiology 36, 9-17 (2003).

167 Armstrong, W. M. Surface active agents and cellular metabolism. I. The effect of cationic detergents on the production of acid and of carbon dioxide by baker's yeast. Archives of biochemistry and biophysics 71, 137-147 (1957).

168 Scharff, T. G. \& Beck, J. L. Effects of Surface-Active Agents on Carbohydrate Metabolism in Yeast. Proceedings of the Society for Experimental Biology and Medicine 100, 307-311 (1959).

169 Goddard, E. D. \& Gruber, J. V. Principles of polymer science and technology in cosmetics and personal care. (CRC Press, 1999).

170 Wille, J. \& Kydonieus, A. Palmitoleic acid isomer (C16: 1 $\Delta 6)$ in human skin sebum is effective against gram-positive bacteria. Skin Pharmacology and Physiology 16, 176-187 (2003).

171 Tran, T.-Q.-M. et al. Bactericidal Effect of Lauric Acid-Loaded PCL-PEG-PCL Nano-Sized Micelles on Skin Commensal Propionibacterium acnes. Polymers 8, 321 (2016). 
172 Silva, E. L., de Carvalho, M., Santos, S. \& Ferreira, L. Solid lipid nanoparticles loaded with retinoic acid and lauric acid as an alternative for topical treatment of acne vulgaris J. Nanosci. Nanotechnol 14, 1-8 (2014).

173 Iwata, H. \& Shimada, K. in Formulas, Ingredients and Production of Cosmetics 21-86 (Springer, 2013).

174 Kabara, J. J. Chemistry and biology of monoglycerides in cosmetic formulations. Glycerine: A Key Cosmetic Ingredient (2018).

175 Bergsson, G., Arnfinnsson, J., SteingrÍmsson, Ó. \& Thormar, H. Killing of Grampositive cocci by fatty acids and monoglycerides. Apmis 109, 670-678 (2001).

176 Guthery, B. E. (Google Patents, 2014).

177 Kabara, J. J. GRAS antimicrobial agents for cosmetic products. J. Soc. Cosmet. Chem 31, 1-10.

178 Kitahara, T. et al. Antimicrobial activity of saturated fatty acids and fatty amines against methicillin-resistant Staphylococcus aureus. Biological and Pharmaceutical Bulletin 27, 1321-1326 (2004).

179 Varothai, S., Nitayavardhana, S. \& Kulthanan, K. Moisturizers for patients with atopic dermatitis. Asian pacific journal of allergy and immunology 31, 91 (2013).

180 Verallo-Rowell, V. M., Dillague, K. M. \& Syah-Tjundawan, B. S. Novel Antibacterial and Emollient Effects of Coconut and Virgin Olive Oils in Adult Atopic Dermatitis. Dermatitis 19, 308-315, doi:10.2310/6620.2008.08052 (2008).

181 Loung, F. S., Silalahi, J. \& Suryanto, D. Antibacterial activity of enzymatic hydrolyzed of virgin coconut oil and palm kernel oil against Staphylococcus aureus, Salmonella thypi and Escherichia coli. International Journal of PharmTech Research 6, 628-633 (2014).

182 Zha, B. et al. Production of glycerol monolaurate-enriched monoacylglycerols by lipase-catalyzed glycerolysis from coconut oil. European Journal of Lipid Science and Technology 116, 328-335, doi:10.1002/ejlt.201300243 (2014).

183 Lin, Y.-C. et al. Glycerol monolaurate and dodecylglycerol effects on Staphylococcus aureus and toxic shock syndrome toxin-1 in vitro and in vivo. PloS one 4, e7499-e7499, doi:10.1371/journal.pone.0007499 (2009). 
184 Schlievert, P. M., Kilgore, S. H., Seo, K. S. \& Leung, D. Y. M. Glycerol Monolaurate Contributes to the Antimicrobial and Anti-inflammatory Activity of Human Milk. Scientific Reports 9, 14550, doi:10.1038/s41598-019-51130-y (2019).

185 Peterson, M. L. \& Schlievert, P. M. Glycerol Monolaurate Inhibits the Effects of Gram-Positive Select Agents on Eukaryotic Cells. Biochemistry 45, 2387-2397, doi:10.1021/bi051992u (2006).

186 Houtman Jon, C. D. et al. Characterizing how glycerol monolaurate (GML) affects human T cell signaling and function, University of Iowa, (2018).

187 Rajani, P. S., Seppo, A. E. \& Järvinen, K. M. Immunologically active components in human milk and development of atopic disease, with emphasis on food allergy, in the pediatric population. Frontiers in pediatrics 6, 218 (2018).

188 Bhalla, T., Kumar, V. \& Bhatia, S. Hydroxy acids: production and applications. Advances in Industrial Biotechnology. IK International Publishing House Pvt Ltd, India, 56-76 (2013).

189 Jack, D. B. One hundred years of aspirin. The Lancet 350, 437-439 (1997).

190 Goldberg, D. J. Facial rejuvenation: a total approach. (Springer Science \& Business Media, 2007).

191 Datta, H. S., Mitra, S., Paramesh, R. \& Patwardhan, B. Theories and management of aging: modern and ayurveda perspectives. Evidence-Based Complementary and Alternative Medicine 2011 (2011).

192 Martin, M. Selling Beauty: Cosmetics, Commerce, and French Society, 1750-1830. (JHU Press, 2009).

193 Annales de chimie et de physique. v. (1816).

194 Annalen der Chemie und Pharmacie. 136 v. (1840).

195 Kornhauser, A., Coelho, S. G. \& Hearing, V. J. Applications of hydroxy acids: classification, mechanisms, and photoactivity. Clinical, cosmetic and investigational dermatology: CCID 3, 135 (2010).

196 Surivet, J.-P. \& Vatèle, J.-M. Total synthesis of antitumor Goniothalamus styryllactones. Tetrahedron 55, 13011-13028 (1999).

197 Furlenmeier, A., Quitt, P., Vogler, K. \& Lanz, P. (Google Patents, 1976). 
198 Terreni, M. et al. Modulation of penicillin acylase properties via immobilization techniques: one-pot chemoenzymatic synthesis of cephamandole from cephalosporin C. Bioorganic \& medicinal chemistry letters 11, 2429-2432 (2001).

199 Moghimipour, E. Hydroxy Acids, the Most Widely Used Anti-aging Agents. Jundishapur J Nat Pharm Prod 7, 9-10 (2012).

200 Wang, X. A theory for the mechanism of action of the $\alpha$-hydroxy acids applied to the skin. Medical hypotheses 53, 380-382 (1999).

201 Babilas, P., Knie, U. \& Abels, C. Cosmetic and dermatologic use of alpha hydroxy acids. JDDG: Journal der Deutschen Dermatologischen Gesellschaft 10, 488-491 (2012).

202 Green, B. After 30 years... the future of hydroxyacids. Journal of cosmetic dermatology 4, 44-45 (2005).

203 SCCNFP. Opinion of the Scientific Committee on Cosmetic Products and NonFood Products Intended for Consumers Concerning Salicylic Acid. (2002).

204 Furman, B. L. in Reference Module in Biomedical Sciences (Elsevier, 2018).

205 Zeichner, J. A. The Use of Lipohydroxy Acid in Skin Care and Acne Treatment. J Clin Aesthet Dermatol 9, 40-43 (2016).

206 Saint-Léger, D., Lévêque, J. L. \& Verschoore, M. The use of hydroxy acids on the skin: characteristics of C8-lipohydroxy acid. Journal of cosmetic dermatology $\mathbf{6}$, 59-65 (2007).

207 Smith, W. Comparative effectiveness of $\alpha$-hydroxy acids on skin properties. International journal of cosmetic science 18, 75-83 (1996).

208 Moy, L. S., Murad, H. \& Moy, R. L. Glycolic acid peels for the treatment of wrinkles and photoaging. The Journal of dermatologic surgery and oncology 19, 243-246 (1993).

209 Fisher, G. J. et al. Mechanisms of photoaging and chronological skin aging. Archives of dermatology 138, 1462-1470 (2002).

210 Bernstein, E. F., Lee, J., Brown, D. B., Yu, R. \& Van Scott, E. Glycolic acid treatment increases type I collagen mRNA and hyaluronic acid content of human skin. Dermatologic surgery 27, 429-433 (2001). 
211 Atzori, L., Brundu, M., Orru, A. \& Biggio, P. Glycolic acid peeling in the treatment of acne. Journal of the European Academy of Dermatology and Venereology 12, 119-122 (1999).

212 Abels, C. et al. A 10\% glycolic acid containing oil-in-water emulsion improves mild acne: a randomized double-blind placebo-controlled trial. Journal of cosmetic dermatology 10, 202-209 (2011).

213 Takenaka, Y., Hayashi, N., Takeda, M., Ashikaga, S. \& Kawashima, M. Glycolic acid chemical peeling improves inflammatory acne eruptions through its inhibitory and bactericidal effects on Propionibacterium acnes. The Journal of dermatology 39, 350-354 (2012).

214 Bergfeld, W. F. et al. Safety Assessment of Alpha Hydroxy Acids as Used in Cosmetics: Re-Review. (2013).

215 DiNARDO, J. C., Grove, G. L. \& Moy, L. S. Clinical and histological effects of glycolic acid at different concentrations and $\mathrm{pH}$ levels. Dermatologic surgery 22 , 421-424 (1996).

216 Becker, F., Langford, F., Rubin, M. \& Speelman, P. A histological comparison of $50 \%$ and $70 \%$ glycolic acid peels using solutions with various pHs. Dermatologic surgery 22, 463-465 (1996).

217 Southwest, C. in The Innovation Blog.

218 Danby, S. G. \& Cork, M. J. in pH of the Skin: Issues and Challenges Vol. 54 95107 (Karger Publishers, 2018).

219 Hachem, J.-P. et al. Acute acidification of stratum corneum membrane domains using polyhydroxyl acids improves lipid processing and inhibits degradation of corneodesmosomes. Journal of investigative dermatology 130, 500-510 (2010).

220 Man, M.-Q. et al. Characterization of a hapten-induced, murine model with multiple features of atopic dermatitis: structural, immunologic, and biochemical changes following single versus multiple oxazolone challenges. Journal of Investigative Dermatology 128, 79-86 (2008).

221 Lee, N. R. et al. Application of Topical Acids Improves Atopic Dermatitis in Murine Model by Enhancement of Skin Barrier Functions Regardless of the Origin of Acids. Ann Dermatol 28, 690-696, doi:10.5021/ad.2016.28.6.690 (2016). 
222 Inoue, H., Someno, T., Kawada, M. \& Ikeda, D. Citric acid inhibits a bacterial ceramidase and alleviates atopic dermatitis in an animal model. The Journal of antibiotics 63, 611-613 (2010).

223 Kessler, E., Flanagan, K., Chia, C., Rogers, C. \& ANNA GLASER, D. Comparison of $\alpha$-and $\beta$-Hydroxy Acid Chemical Peels in the Treatment of Mild to Moderately Severe Facial Acne Vulgaris. Dermatologic surgery 34, 45-51 (2008).

224 Prajapati, H. N., Dalrymple, D. M. \& Serajuddin, A. T. M. A Comparative Evaluation of Mono-, Di- and Triglyceride of Medium Chain Fatty Acids by Lipid/Surfactant/Water Phase Diagram, Solubility Determination and Dispersion Testing for Application in Pharmaceutical Dosage Form Development. Pharmaceutical Research 29, 285-305, doi:10.1007/s11095-011-0541-3 (2012).

225 WEISS, S. C. Conventional topical delivery systems. Dermatologic Therapy 24, 471-476, doi:10.1111/j.1529-8019.2012.01458.x (2011).

226 Rosen, M. Intelligent Delivery Systems for Enhancing the Performance of Active Ingredients in Skin Care Formulations, $<$ https://www.experts.com/articles/intelligent-delivery-systems-activeingredients-skin-care-formulations-by-meyer-rosen $>($

227 Hirva, S. \& Jenisha, P. Bicelle: a lipid nanostructure for transdermal delivery. $J$ Crit Rev 3, 17-22 (2016).

228 Rodríguez, G. et al. Bicelles: new lipid nanosystems for dermatological applications. Journal of biomedical nanotechnology 11, 282-290 (2015).

229 Barbosa-Barros, L. et al. Bicelles: lipid nanostructured platforms with potential dermal applications. Small 8, 807-818 (2012).

230 Rodríguez, G. et al. in Soft Nanoparticles for Biomedical Applications 280-311 (Royal Society of Chemistry, 2014).

231 Alexeyev, O. A. Bacterial landscape of human skin: seeing the forest for the trees. Experimental dermatology 22, 443-446 (2013).

232 Wilbur, R. L. (Gensco Pharma, Miami, 2017).

$233 \mathrm{Xu}, \mathrm{J}$. et al. Bicelle-Enabled Structural Studies on a Membrane-Associated Cytochrome b5 by Solid-State MAS NMR Spectroscopy. Angewandte Chemie International Edition 47, 7864-7867 (2008). 
234 Sanders, C. R. \& Schwonek, J. P. Characterization of magnetically orientable bilayers in mixtures of dihexanoylphosphatidylcholine and dimyristoylphosphatidylcholine by solid-state NMR. Biochemistry 31, 8898-8905 (1992).

235 Sanders, C. R. \& Prosser, R. S. Bicelles: a model membrane system for all seasons? Structure 6, 1227-1234 (1998).

236 Kaul, S., Gulati, N., Verma, D., Mukherjee, S. \& Nagaich, U. Role of Nanotechnology in Cosmeceuticals: A Review of Recent Advances. J Pharm (Cairo) 2018, 3420204-3420204, doi:10.1155/2018/3420204 (2018).

237 Barbosa-Barros, L. et al. Effect of bicellar systems on skin properties. International journal of pharmaceutics 352, 263-272 (2008).

238 Dubey, V. et al. Dermal and transdermal delivery of an anti-psoriatic agent via ethanolic liposomes. Journal of controlled release 123, 148-154 (2007).

239 Sheu, M.-T., Chen, S.-Y., Chen, L.-C. \& Ho, H.-O. Influence of micelle solubilization by tocopheryl polyethylene glycol succinate (TPGS) on solubility enhancement and percutaneous penetration of estradiol. Journal of controlled release $\mathbf{8 8}, 355-368$ (2003).

240 Nafisi, S. \& Maibach, H. I. in Emerging Nanotechnologies in Immunology (eds Ranjita Shegokar \& Eliana B. Souto) 47-88 (Elsevier, 2018).

241 Maghraby, G. M. E., Williams, A. C. \& Barry, B. W. Can drug-bearing liposomes penetrate intact skin? Journal of Pharmacy and Pharmacology 58, $415-429$ (2006).

242 Berardesca, E., Vignoli, G. P., Distante, F., Brizzi, P. \& Rabbiosi, G. Effects of water temperature on surfactant-induced skin irritation. Contact dermatitis 32, 8387 (1995).

243 Struppe, J., Whiles, J. A. \& Vold, R. R. Acidic phospholipid bicelles: a versatile model membrane system. Biophysical journal 78, 281-289 (2000).

244 Rubio, L. et al. Bicellar systems as new delivery strategy for topical application of flufenamic acid. International journal of pharmaceutics 444, 60-69 (2013).

245 Barbosa-Barros, L. et al. Penetration and growth of DPPC/DHPC bicelles inside the stratum corneum of the skin. Langmuir 24, 5700-5706 (2008). 
246 Purnamawati, S., Indrastuti, N., Danarti, R. \& Saefudin, T. The Role of Moisturizers in Addressing Various Kinds of Dermatitis: A Review. Clin Med Res 15, 75-87, doi:10.3121/cmr.2017.1363 (2017).

247 Rodríguez, G. et al. A unique bicellar nanosystem combining two effects on stratum corneum lipids. Molecular pharmaceutics 9, 482-491 (2012).

248 Rubio, L. et al. Bicellar systems for in vitro percutaneous absorption of diclofenac. International journal of pharmaceutics 386, 108-113 (2010).

249 Rodríguez, G. et al. Bicosomes: bicelles in dilute systems. Biophysical journal 99, 480-488 (2010).

250 Barbosa-Barros, L., De la Maza, A., Walther, P., Estelrich, J. \& López, O. Morphological effects of ceramide on DMPC/DHPC bicelles. Journal of microscopy 230, 16-26 (2008).

251 Fernández, E. et al. Bicelles and bicosomes as free radical scavengers in the skin. RSC advances 4, 53109-53121 (2014).

252 Mäler, L. \& Gräslund, A. in Macromolecular Drug Delivery 129-139 (Springer, 2009).

253 De Angelis, A. A. \& Opella, S. J. Bicelle samples for solid-state NMR of membrane proteins. Nature protocols 2, 2332 (2007).

254 Triba, M. N., Warschawski, D. E. \& Devaux, P. F. Reinvestigation by phosphorus NMR of lipid distribution in bicelles. Biophysical journal 88, 1887-1901 (2005).

255 Elias, P. M. et al. Basis for abnormal desquamation and permeability barrier dysfunction in RXLI. Journal of investigative dermatology 122, 314-319 (2004).

256 Sanders II, C. R., Hare, B. J., Howard, K. P. \& Prestegard, J. H. Magneticallyoriented phospholipid micelles as a tool for the study of membrane-associated molecules. Progress in Nuclear Magnetic Resonance Spectroscopy 26, 421-444 (1994).

257 Ujwal, R. \& Abramson, J. High-throughput crystallization of membrane proteins using the lipidic bicelle method. JoVE (Journal of Visualized Experiments), e3383 (2012).

258 Kimble-Hill, A. C. A review of factors affecting the success of membrane protein crystallization using bicelles. Frontiers in biology 8, 261-272 (2013). 
259 Marcotte, I. \& Auger, M. Bicelles as model membranes for solid-and solution-state NMR studies of membrane peptides and proteins. Concepts in Magnetic Resonance Part A: An Educational Journal 24, 17-37 (2005).

260 Ram, P. \& Prestegard, J. Magnetic field induced ordering of bile salt/phospholipid micelles: new media for NMR structural investigations. Biochimica et Biophysica Acta (BBA)-Biomembranes 940, 289-294 (1988).

261 Yamamoto, K., Soong, R. \& Ramamoorthy, A. Comprehensive analysis of lipid dynamics variation with lipid composition and hydration of bicelles using nuclear magnetic resonance (NMR) spectroscopy. Langmuir 25, 7010-7018 (2009).

262 Surya, W., Li, Y., Millet, O., Diercks, T. \& Torres, J. Transmembrane and juxtamembrane structure of $\alpha \mathrm{L}$ integrin in bicelles. PloS one 8 (2013).

263 van Dam, L., Karlsson, G. \& Edwards, K. Direct observation and characterization of DMPC/DHPC aggregates under conditions relevant for biological solution NMR. Biochimica et Biophysica Acta (BBA)-Biomembranes 1664, 241-256 (2004).

264 Barbosa-Barros, L. et al. Use of high-pressure freeze fixation and freeze fracture electron microscopy to study the influence of the phospholipid molar ratio in the morphology and alignment of bicelles. Journal of microscopy 233, 35-41 (2009).

265 Studer, D., Graber, W., Al-Amoudi, A. \& Eggli, P. A new approach for cryofixation by high-pressure freezing. Journal of microscopy 203, 285-294 (2001).

266 Rodríguez, G. et al. Characterization of new DOPC/DHPC platform for dermal applications. European Biophysics Journal 42, 333-345 (2013).

267 Severs, N. J. Freeze-fracture electron microscopy. Nature Protocols 2, 547-576, doi:10.1038/nprot.2007.55 (2007).

268 The principles of dynamic light scattering, <https:/wiki.anton-paar.com/en/theprinciples-of-dynamic-light-scattering/\#c39795 > (

269 Cócera, M. et al. Characterisation of skin states by non-crystalline diffraction. Soft Matter 7, 8605-8611 (2011).

270 Loudet, C. et al. Biphenyl bicelle disks align perpendicular to magnetic fields on large temperature scales: a study combining synthesis, solid-state NMR, TEM, and SAXS. Biophysical journal 92, 3949-3959 (2007). 
271 Costa, M. et al. in Applications of Synchrotron Light to Scattering and Diffraction in Materials and Life Sciences 265-280 (Springer, 2009).

272 Nieh, M.-P., Glinka, C. J., Krueger, S., Prosser, R. S. \& Katsaras, J. SANS study on the effect of lanthanide ions and charged lipids on the morphology of phospholipid mixtures. Biophysical journal 82, 2487-2498 (2002).

273 Fisher, Z., Jackson, A., Kovalevsky, A., Oksanen, E. \& Wacklin, H. in Experimental Methods in the Physical Sciences Vol. 49 (eds Felix FernandezAlonso \& David L. Price) 1-75 (Academic Press, 2017).

274 Luchette, P. A. et al. Morphology of fast-tumbling bicelles: a small angle neutron scattering and NMR study. Biochimica et Biophysica Acta (BBA) - Biomembranes 1513, 83-94, doi:https://doi.org/10.1016/S0005-2736(01)00358-3 (2001).

275 Dwivedi, D. \& Lepková, K. SAXS and SANS Techniques for Surfactant Characterization: Application in Corrosion Science. Application and Characterization of Surfactants, 287 (2017).

276 Lin, T. L., Liu, C. C., Roberts, M. F. \& Chen, S. H. Structure of mixed short-chain lecithin/long-chain lecithin aggregates studied by small-angle neutron scattering. The Journal of Physical Chemistry 95, 6020-6027 (1991).

277 Caldwell, T. A. et al. Low- q Bicelles Are Mixed Micelles. J Phys Chem Lett 9, 4469-4473, doi:10.1021/acs.jpclett.8b02079 (2018).

278 Breyton, C. et al. Small angle neutron scattering for the study of solubilised membrane proteins. The European Physical Journal E 36, 71 (2013).

279 Erman, B. \& Mark, J. E. in The Science and Technology of Rubber (Fourth Edition) (eds James E. Mark, Burak Erman, \& C. Michael Roland) 167-192 (Academic Press, 2013).

280 Coderch, L. et al. The effect of liposomes on skin barrier structure. Skin Pharmacology and Physiology 12, 235-246 (1999).

281 Lima, C. A., Goulart, V. P., Côrrea, L., Pereira, T. M. \& Zezell, D. M. ATR-FTIR spectroscopy for the assessment of biochemical changes in skin due to cutaneous squamous cell carcinoma. International journal of molecular sciences 16, 66216630, doi:10.3390/ijms16046621 (2015). 
282 Rodriguez, G. et al. Conformational changes in stratum corneum lipids by effect of bicellar systems. Langmuir 25, 10595-10603 (2009).

283 Almeida, P. F., Vaz, W. L. \& Thompson, T. Lateral diffusion in the liquid phases of dimyristoylphosphatidylcholine/cholesterol lipid bilayers: a free volume analysis. Biochemistry 31, 6739-6747 (1992).

284 Fluhr, J. W., Feingold, K. R. \& Elias, P. M. Transepidermal water loss reflects permeability barrier status: validation in human and rodent in vivo and ex vivo models. Experimental dermatology 15, 483-492 (2006).

285 Bazin, R. \& Fanchon, C. Equivalence of face and volar forearm for the testing of moisturizing and firming effect of cosmetics in hydration and biomechanical studies. International journal of cosmetic science 28, 453-461 (2006).

286 Edwards, C. \& Marks, R. Evaluation of biomechanical properties of human skin. Clinics in dermatology 13, 375-380 (1995).

287 Luthra, A. Complications of Mesotherapy. Complications in Cosmetic Dermatology: Crafting Cures, 143 (2016).

288 Vist, M. R. \& Davis, J. H. Phase equilibria of cholesterol/dipalmitoylphosphatidylcholine mixtures: deuterium nuclear magnetic resonance and differential scanning calorimetry. Biochemistry 29, 451-464 (1990).

289 Ollivon, M., Lesieur, S., Grabielle-Madelmont, C. \& Paternostre, M. t. Vesicle reconstitution from lipid-detergent mixed micelles. Biochimica et Biophysica Acta (BBA)-Biomembranes 1508, 34-50 (2000).

290 Triba, M. N., Devaux, P. F. \& Warschawski, D. E. Effects of lipid chain length and unsaturation on bicelles stability. A phosphorus NMR study. Biophysical journal 91, 1357-1367 (2006).

291 Katsaras, J., Harroun, T. A., Pencer, J. \& Nieh, M.-P. "Bicellar" lipid mixtures as used in biochemical and biophysical studies. Naturwissenschaften 92, 355-366 (2005).

292 Nieh, M.-P., Raghunathan, V., Wang, H. \& Katsaras, J. Highly aligned lamellar lipid domains induced by macroscopic confinement. Langmuir 19, 6936-6941 (2003). 
293 Barbosa-Barros, L., De La Maza, A., López-Iglesias, C. \& López, O. Ceramide effects in the bicelle structure. Colloids and Surfaces A: Physicochemical and Engineering Aspects 317, 576-584 (2008).

294 Pappas, A. Epidermal surface lipids. Dermatoendocrinol 1, 72-76, doi:10.4161/derm.1.2.7811 (2009).

295 Yokomizo, Y. \& Sagitani, H. The effects of phospholipids on the percutaneous penetration of indomethacin through the dorsal skin of guinea pig in vitro. 2 . The effects of the hydrophobic group in phospholipids and a comparison with general enhancers. Journal of controlled release 42, 37-46 (1996).

296 Ulrich, A. S., Sami, M. \& Watts, A. Hydration of DOPC bilayers by differential scanning calorimetry. Biochimica et Biophysica Acta (BBA)-Biomembranes 1191, 225-230 (1994).

297 Johansson, E., Lundquist, A., Zuo, S. \& Edwards, K. Nanosized bilayer disks: Attractive model membranes for drug partition studies. Biochimica et Biophysica Acta (BBA) - Biomembranes 1768, 1518-1525, doi:https://doi.org/10.1016/j.bbamem.2007.03.006 (2007).

298 Johnsson, M. \& Edwards, K. Liposomes, disks, and spherical micelles: aggregate structure in mixtures of gel phase phosphatidylcholines and poly (ethylene glycol)phospholipids. Biophysical Journal 85, 3839-3847 (2003). 


\section{Chapter 3}

\section{Experimental Methodology}

The characterization of antimicrobial lipids, alpha hydroxy acid and bicelles is crucial to move forward in the development of a mild, yet highly effective formulation for acne and atopic dermatitis. The following chapter focuses on discussing the rationale behind the selection of the experimental techniques and their principles. It provides an introductory explanation of how the chemical, physical and biological essays were integrated; followed by the milestones of each result chapter and the appropriate techniques. The materials, synthesis and protocol for the methods are described exactly as conducted. The aim is that this chapter shows the acquired knowledge needed to perform: Fluorescence Spectroscopy, QCM-D, Fluorescence Microscopy, MIC, MBC, Confocal Microscopy, Bioluminescence measurements, DLS, cryo-TEM and Cell viability tests. 


\subsection{Rationale for the Selection of Materials and Experimental Techniques}

This chapter focuses on describing in detail the experimental methodologies used to characterize the antimicrobial lipids, the alpha hydroxy acid (glycolic acid) and the bicelles. It is split in three main sections: materials, materials synthesis and experimental techniques. The core motivation behind the selection of the experimental approaches is the integrations of the physicochemical, biophysical and biological methodologies to make connections and associative conclusions, and hence, provide a better solution for the skin diseases at matter. In most drug screening investigations, the focus is either biological, invitro/in-vivo, or chemical, leading unanswered questions and a great gap between them. Just recently, multi-sided publications have aided to decipher underlying mechanistic features and improve strategies to monitor modes of action in real time. As an example, the publication of Yoon et al. characterized the biological and biophysical properties of lauric acid and glycerol monolaruate ${ }^{1}$, easing the further assessment of the compounds. On the other hand, publications related to delivery methods do provide integrative insights of the biological and physical features. Barbosa-Barros et al. characterized the morphology of bicelles with physical and imaging techniques, to further employ this knowledge and try them in-vitro on skin tissue and in-vivo on healthy volunteers ${ }^{2}$. The narrated modes of conceptualization guided the entire way of working in this thesis.

The overall hypothesis of the project was that antimicrobial lipids assembled as bicelles in combination with the hydroxy acid can be used to inhibit $C$. acnes and $S$. aureus, bacterium implicated in acne and atopic dermatitis pathogenesis, respectively. The selection of the methods for each result chapter was led by their independent milestones. For chapter 4, the aim was to assess the effect of lauric acid and glycerol monolaurate treatment against supported lipid bilayers (SLBs) and C. acnes in acidic $\mathrm{pH}$ conditions mimicking the skin environment. Chapter 5, focused on determining whether glycolic acid can inhibit C. acnes, also under acidic conditions; and how it works. Chapter 6, compared the potency of glycerol monolaurate assembled as a bicelle with a more straightforward, yet of great relevance, bacterial model, S. aureus. 
The first section, materials, lists and specifies the chemical and biological reagents. For the single chain lipid amphiphiles the fatty acid lauric acid and its monoglyceride glycerol monolaurate are detailed. These medium chain saturated lipids have the highest proven antibacterial activity against $C$. acnes and $S$. aureus. The next compound is the organic acid, glycolic acid, which is the smallest alpha hydroxy acid and is broadly applied for chemical peeling procedures and unclogging pores. The third group of reagents are the phospholipids DOPC and DHPC, used to make bicelle delivery vehicles. Behind, the buffers, bacterial cell strains and antibacterial testing consumables are listed and described copiously.

The second section, materials synthesis, comprises the preparation of antimicrobial lipid solutions, glycolic acid, bicelles and bacterial cell culture. The formulation of the antimicrobial lipids was standardized for all assays. Glycolic acid solutions were identically prepared, though, the $\mathrm{pH}$ values were adjusted according to the tested value. For the bicelles three different q-ratios of the same composition DOPC/glycerol monolaurate were made: $0.05,0.25$ and 2.5. DOPC/DHPC bicelles were prepared for the fabrication of supported lipid bilayers (SLBs). As to the biological assays, quality control strains of $C$. acnes and $S$. aureus were cultured with the pertinent biological reagents. A thorough explanation of the methods, highlighting the key steps in the experimental execution and the justification of the design are given.

The last section, experimental techniques, points out the fundamentals, the rationale behind the selection and the protocols. For chapter 4, fluorescence spectroscopy measurements were conducted to assess the molecular self-assembly of the antimicrobial lipids by means of the critical micelle concentration (CMC) in appropriate $\mathrm{pH}$ conditions (physiological, 7.4, and skin-like, 4.5). The $\mathrm{pK}_{\mathrm{a}}$ value of the fatty acid lauric acid was measured to further match it with its antibacterial behavior, dictated by the concept of higher antibacterial activity with protonated molecules. The value was not obtained for the monoglyceride, glycerol monolaurate, since its ionization state remains unaltered in all $\mathrm{pH}$ ranges. To study how antimicrobial lipids interact with phospholipids, main components of cell membranes, supported lipid bilayers (SLB) were used as a replacement for real-time evaluation of the 
morphological response. The concentration-dependent effect of the compounds was tracked with the change in mass and viscoelasticity of the SLB platform in a quartz crystal microbalance-dissipation (QCM-D). This move was done to dodge the major drawbacks of biological probes at the first stages of a project and limit the number of feasible permeating candidates. To complement the QCM-D experiments, fluorescence microscopy measurements were collected to visually observe the morphological changes. Together, these experiments provided a deep insight of the disruptive behavior. Lastly, biological assays were performed to determine how $\mathrm{pH}$-dependent conditions affect the inhibitory activity of antimicrobial lipids against $C$. acnes, the target bacterium in the chapter. In chapter 5 , the screening of the inhibitory concentration of glycolic acid (GA) against $C$. acnes was done with an antibacterial test, minimum inhibitory concentration (MIC). Next, enumeration essays to evaluate the concentration range at which GA kills $C$. acnes (by at least $99.99 \%$ ) in different $\mathrm{pH}$ conditions, was obtained with the minimum bactericidal concentration $(\mathrm{MBC})$ test. To confirm cell killing confocal microscopy imaging was performed to distinguish live and dead cells. The permeation effect of the cell membrane was recorded by monitoring the release of adenosine triphosphate (ATP), which is a sensitive marker of membrane damage. For the ultimate chapter concerning experimental results, chapter 6, the bactericidal concentration (MBC) of the DOPC/GML bicelles was ascertained to corroborate its feasibility as a reliable antibacterial treatment. The results were further complemented with confocal microscopy images taken from bacterial counterstaining, using the LIVE/DEAD kit. A deep biophysical characterization was done to verify the membrane disruptive activity earlier described from GML over a SLB utilizing Q-CMD. Additionally, the possible formation of a SLB according to the ratio of long and short chain lipids (DOPC/GML) was determined. The morphological characterization of the bicellar sample was accomplished with dynamic light scattering and cryo-TEM. The first one to figure out the apparent size and dispersity of the bicelles, the second, to have a visual proof. Finally, the cell proliferation and viability were tested over human immortalized keratinocyte cells $(\mathrm{HaCaT})$ to prove its safeness. 


\subsection{Materials}

\subsubsection{Single Chain Lipid Amphiphiles}

Lauric acid (LA) was purchased from Sigma-Aldrich (St. Louis, MO) and glycerol monolaurate (GML) from Abcam (Cambridge, UK). The two compounds have saturated hydrocarbon chains, which confer them a high chemical stability in ambient settings. Both reagents were stored at room temperature and prepared right before testing as recommended by the manufacturer.

\subsubsection{Organic Acid}

Glycolic acid was obtained from Sigma-Aldrich (St. Louis, MO). The storage conditions were at room temperature. Sample weighing and preparation were done immediately before each experiment.

\subsubsection{Phospholipid Reagents}

1,2-Dioleoyl-sn-glycero-3-phosphocholine (DOPC) and 1,2-dihexanoyl-sn-glycero-3phosphocholine (DHPC) were acquired as-supplied in chloroform from Avanti Polar Lipids (Alabaster, AL). The storage conditions were at $-20{ }^{\circ} \mathrm{C}$ and new stock solutions were prepared for all tests.

\subsubsection{Buffer Reagents}

Phosphate-buffered saline (PBS) was bought from Gibco (Carlsbad, CA). It consist of 1.54 $\mathrm{mM} \mathrm{KH} 2 \mathrm{PO}_{4}, 155.17 \mathrm{mM} \mathrm{NaCl}$ and $2.71 \mathrm{mM} \mathrm{Na}_{2} \mathrm{HPO}_{4}-7 \mathrm{H}_{2} \mathrm{O}$. PBS buffer is preferred in biological experiments due to its physiological $\mathrm{pH}(\sim 7.4)^{3}$ and salt ions, which maintain the osmolarity of the bacterial cells ${ }^{4}$. PBS was autoclaved for all antibacterial test at 121 ${ }^{\circ} \mathrm{C}$ for 15 mins and kept at $4{ }^{\circ} \mathrm{C}$ under sterile conditions. 


\subsubsection{Bacterial Cell Strains and Antibacterial Testing Reagents}

Two different bacterial species, Cutibacterium acnes (ATCC 11827) and Staphylococcus aureus (ATCC 25923) were procured from American Type Culture Collection (Manassas, VA). The reason to select these bacteria strains is their common use in quality control and large amount of documented data ${ }^{5-7}$. The bacteria were supplied lyophilized and further propagated by suspending them in Tryptic soy broth (TSB) for C. acnes and Luria Bertani (LB) broth for $S$. aureus. Tryptic soy broth (Becton Dickinson, Franklin Lakes, NJ) consist of tryptone $(17 \mathrm{~g} / \mathrm{l})$, soytone $(3 \mathrm{~g} / \mathrm{l})$, glucose $(2.5 \mathrm{~g} / \mathrm{l})$, sodium chloride $(5 \mathrm{~g} / \mathrm{l})$ and dipotassium phosphate $(2.5 \mathrm{~g} / \mathrm{l})$. Luria Bertani broth (BD, Sparks, MD) is prepared with tryptone $(10 \mathrm{~g} / \mathrm{l})$, yeast extract $(5 \mathrm{~g} / \mathrm{l})$ and sodium chloride $(10 \mathrm{~g} / \mathrm{l})$. After propagation, stocks for both bacteria were prepared by suspending them in glycerol, $50 \% \mathrm{v} / \mathrm{v}$, and stowing them in $-80{ }^{\circ} \mathrm{C}$. TSB with $5 \%$ defibrinated sheep blood (Thermo Fisher Scientific, Waltham, MA) was used in all bacterial cell culture experiments with C. acnes in chapter 4 and 5. The cultivation of this oxygen-free bacteria was inside an anaerobic incubation container (BD GasPak EZ) where a $\mathrm{CO}_{2}$-enriched environment was produced with anaerobe system sachets (GasPak EZ Anaerobe System sachets with indicator). The anaerobe system (Gas-Pak) composed by the incubation container and the anaerobe sachets were purchased from Becton Dickinson (Franklin Lakes, NJ). The bacterial cell density was assessed with optical density measurements at $600 \mathrm{~nm}\left(\mathrm{OD}_{600}\right)$ using TSB without blood to avoid false positive readouts. The minimum inhibitory concentration (MIC) and minimum bactericidal concentration (MBC) for $C$. acnes tests were assessed after bacteria were streaked on tryptic soy agar base plates with 5\% sheep blood from Hardy Diagnostics (Santa Maria, CA). Blood agar plates are made with pancreatic digest of casein $(15 \mathrm{~g} / 1)$, peptic digest of soybean meal (5 g/l), sodium chloride $(5 \mathrm{~g} / \mathrm{l})$, sheep blood $(50 \mathrm{ml})$ and agar $(12 \mathrm{~g} / \mathrm{l})$. Regarding to $S$. aureus, LB broth was used as culture media and medium for optical density measurements $\left(\mathrm{OD}_{600}\right)$. In chapter 6 , the $\mathrm{MIC}$ and $\mathrm{MBC}$ tested samples with $S$. aureus were platted in Mueller-Hinton (MH) agar (Sigma-Aldrich, St Louis, MO). The composition of MH agar is beef infusion solids (2 g/l), starch (1.5 g/l), casein hydrolysate (17.5 g/l), and agar. As for the antimicrobial lipid solutions encompass in chapters 4, 5 and 6 the MIC and MBC assays were prepared in PBS. All media and buffer for bacterial cell culture and 
antimicrobial tests were autoclaved at $121{ }^{\circ} \mathrm{C}$ for $15 \mathrm{mins}$ and stored at $4{ }^{\circ} \mathrm{C}$ under sterile conditions.

\subsection{Materials Synthesis}

\subsubsection{Preparation of Antimicrobial Lipid Solutions}

Saturated fatty acids, like LA, are poorly soluble in aqueous solvents ${ }^{8}$. In several publications they report the use of the "universal solvent" dimethyl sulfoxide (DMSO), by preparing stock solutions of the compound with $5 \% \mathrm{v} / \mathrm{v}$ sulfoxide ${ }^{9-11}$. However, DMSO is a polar aprotic solvent highly avoided for the formulation of skincare products due to its tendency to cause skin rashes ${ }^{12,13}$. For the purpose of this research, antimicrobial lipids were initially dissolved with ethanol and further diluted with PBS to reach a final ethanol concentration of $1 \%{ }^{8,14}$. Low doses of ethanol $(>1 \%)$ were kept through all experiments to elude undesirable effects as skin cell apoptosis, dryness and sensitization ${ }^{15,16}$. Stock solutions of LA and GML were prepared by weighing the appropriate amount of lyophilized compound and dissolving them in ethanol to a concentration of 400 or $200 \mathrm{mM}$, respectively. Before experiment, aliquots were diluted 100-fold with PBS buffer solution to the highest test concentration of $4 \mathrm{mM}$ and $2 \mathrm{mM}$ for LA and GML, respectively. To promote solubilization, the samples were profusely vortexed and heated to $70{ }^{\circ} \mathrm{C}$ for 30 min, then cooled down before measurements were conducted at room temperature.

\subsubsection{Glycolic Acid Preparation}

Glycolic acid is an alpha hydroxy acid soluble in aqueous solvents ${ }^{17}$. The compound is generally dissolved in water, or any other buffer, and $\mathrm{pH}$ is adjusted with $\mathrm{NaOH}^{18}$. In this case, stock solutions of $200 \mathrm{mM}$ glycolic acid were prepared in PBS. The initial solution $\mathrm{pH}$ was around 2.3 and the $\mathrm{pH}$ was adjusted by adding $3 \mathrm{M} \mathrm{NaOH}$ step-wise until reaching the desired sample $\mathrm{pH}$ value. Before experiment, glycolic acid samples were heated at 55 ${ }^{\circ} \mathrm{C}$ for $30 \mathrm{~min}$, and then cooled down before measurements were conducted at room temperature. 


\subsubsection{Bicelles Preparation}

Nearly all bicelle protocols follow the same series of preparations steps, making it simple and highly reproducible. The initial step involves the mixture of the long-chain and shortchain lipids, according to the predetermined q-ratio. Followed by the removal of any trace of organic solvent, typically this procedure is done with a rotary evaporator or a gentle stream of nitrogen together with a complete evaporation under a vacuum desiccator. The mixture of lipids or lipid-detergent is hydrated with the right amount of buffer and submitted to several cycles of freezing and thawing in a hot bath or sonicator ${ }^{19-21}$. In an effort to prepare the lipid systems the methodology published by Kolahdouzan et al. was followed ${ }^{22}$. In glass vials, DOPC and the mixture of DOPC/DHPC in chloroform solvent were evaporated with nitrogen gas until a lipid film on the walls was formed. To ensure the entire removal of the organic solvent the vials were dried overnight in a vacuum desiccator. The stock solution of GML was prepared by weighing the lyophilized compound and dissolving in ethanol to a concentration of $200 \mathrm{mM}$, later diluted with buffer solution (PBS 1X, pH 7.4) to a concentration of $2 \mathrm{mM}$. The desiccated vial with DOPC was hydrated with $1 \mathrm{~mL}$ of the $2 \mathrm{mM}$ GML solution and the DOPC/DHPC mixture with PBS buffer. The obtained lipid suspensions for DOPC/GML had a long-chain phospholipid concentration of $5 \mathrm{mM}, 0.5 \mathrm{mM}$ and $0.1 \mathrm{mM}$ for the q-ratios of $2.5,0.25$ and 0.05 respectively. The DOPC/DHPC bicelles had a DOPC concentration of $0.031 \mathrm{mM}$ for the q-ratio 0.25 . The samples were immersed into liquid nitrogen for $1 \mathrm{~min}$, followed by $5 \mathrm{~min}$ thawing in water bath $\left(60^{\circ} \mathrm{C}\right)$ and vortexing for $30 \mathrm{~s}$. Five cycles of freeze-thaw-vortex were performed. The final bicellar mixture of DOPC/GML q-ratio 2.5 displayed a milky appearance at room temperature. The rest of the samples were clear at plain sight.

\subsubsection{Bacterial Cell Culture}

\section{Cutibacterium acnes}

C. acnes strain ATCC 11827 was cultured in Tryptic soy broth with 5\% defibrinated sheep blood for 48 hrs under anaerobic conditions using a Gas-Pak $\left(80 \% \mathrm{~N}_{2}, 13 \% \mathrm{CO}_{2}, 7 \% \mathrm{H}_{2}\right)$ at $37^{\circ} \mathrm{C}$. The bacterial suspension was then re-inoculated in fresh Tryptic soy broth with $5 \%$ defibrinated sheep blood and cultured under the same anaerobic conditions for an 
additional $24 \mathrm{hrs}$. The bacterial cells were next harvested by centrifugation at $1485 \times \mathrm{g}$ for 10 min, washed thrice with PBS, and re-suspended in the same buffer. The $\mathrm{OD}_{600}$ value was measured and the appropriate dilution was made to reach a value of approximately 0.35 (mid-exponential growth phase) $^{23}$. This value corresponds to a density of $2 \times 10^{8}$ $\mathrm{CFU} / \mathrm{mL}$, as confirmed by $\mathrm{CFU}$ (colony-forming unit) enumeration control experiments. A 100 -fold PBS dilution was performed to dilute the cells to a density of $2 \times 10^{6} \mathrm{CFU} / \mathrm{mL}$. For all experiments, a new aliquot of the already prepared bacteria stock with glycerol $50 \%$ $\mathrm{v} / \mathrm{v}$ at $-80{ }^{\circ} \mathrm{C}$ was utilized. Regarding to $\mathrm{CFU}$ enumeration, the optical density of the bacterial cell culture was measured and adjusted to an $\mathrm{OD}_{600}$ of 0.35 . It was further diluted thrice in a 10-fold series manner with PBS buffer, and $100 \mu$ of each diluent were streaked on tryptic soy agar base plates with $5 \%$ sheep blood (90 mm diameter). Incubation was performed under anaerobic conditions using a Gas-Pak $\left(80 \% \mathrm{~N}_{2}, 13 \% \mathrm{CO}_{2}, 7 \% \mathrm{H}_{2}\right)$ at 37 ${ }^{\circ} \mathrm{C}$ for 4 days. After incubation, the agar plates containing between 25 and 250 bacteria colonies were selected for quantification and the colony-forming units were determined by back-dilution. According to routine control experiments, an $\mathrm{OD}_{600}$ of 0.35 corresponds to a cell density of $\sim 2 \times 10^{8} \mathrm{CFU} / \mathrm{mL}$, and a 200 -times dilutions yields to $1 \times 10^{6} \mathrm{CFU} / \mathrm{mL}$

\section{Staphylococcus aureus}

S. aureus, ATCC 25923 was cultured in LB broth overnight at $37{ }^{\circ} \mathrm{C}$. The overnight suspension was inoculated in fresh LB broth and cultured under aerobic conditions until reaching an $\mathrm{OD}_{600}$ of 0.5 (exponential growth phase). The bacterial cells were next harvested by centrifugation at $1485 \times \mathrm{g}$ for $10 \mathrm{~min}$, washed thrice with PBS, and resuspended in the same buffer. The $\mathrm{OD}_{600}$ value was measured and the appropriate dilution was made to reach a value of $\sim 0.1$, following the CLSI standard method for assessing antimicrobial susceptibility ${ }^{24}$. This value corresponds to a density of $4 \times 10^{7} \mathrm{CFU} \mathrm{mL}^{-1}$, as confirmed by colony-forming unit (CFU) enumeration in control experiments. The corresponding dilutions were made with PBS buffer to reach the required bacterial cell density for each test. As with $C$. acnes, freshly prepared bacteria, from the glycerol stock at $-80{ }^{\circ} \mathrm{C}$, were used for each assay. The $\mathrm{CFU}$ enumeration of $S$. aureus was routinely carried out with each experiment to determine the initial inoculum. The procedure required the adjustment of the cell density to an $\mathrm{OD}_{600}$ of 0.1 , followed by the dilution of the sample 
with PBS buffer, three times in 10-fold increments. Each diluent was streaked onto MH agar plates, $10 \mu \mathrm{l}$ volume (small plates, $50 \mathrm{~mm}$ diameter). Plates were incubated for $24 \mathrm{~h}$ at $37^{\circ} \mathrm{C}$ to quantify and determine the colony-forming units by back-dilution. Agar plates containing 25-250 bacteria cells were eligible for enumeration. Based on controls it was determined that an $\mathrm{OD}_{600}$ of 0.1 was equivalent to a cell density of $\sim 4 \times 10^{7} \mathrm{CFU} \mathrm{mL}^{-1}$ and the subsequent dilution 40-times yield to a cell density of $1 \times 10^{6} \mathrm{CFU} / \mathrm{mL}$

\subsection{Experimental Techniques}

This section of the thesis focus on providing the foundations of the techniques and methods of analysis employed. High emphasis is placed in describing meticulously the optimized protocols used to bring to completion this work.

\subsubsection{Critical Micelle Concentration Determination Assay}

The critical micelle concentration (CMC) is defined as the concentration range above which micelles are formed ${ }^{25}$. Micelles are molecular aggregates form above a narrow concentration range of surfactants. These compounds are amphipathic, with an apolar long hydrocarbon-chain and a polar ionic group. The surface-active compounds self-assemble into micelles by sequestering the hydrophobic long hydrocarbon-chain in the center and allowing the hydrophilic ionic group to be in contact with the solvent ${ }^{26}$. This configurations permit to reach a thermodynamically stable state known as hydrophobic effect ${ }^{27}$. For the purpose of this thesis, it is important to characterize the micellar behavior of single-chain lipid amphiphiles, particularly lauric acid and glycerol monolaurate. Free fatty acids and monoglycerides are well known for destabilizing phospholipid bilayers, components of bacteria cell membranes ${ }^{26,28}$. This behavior can be further boost at concentrations above their corresponding CMC values, as demonstrated by Yoon et al. ${ }^{1}$. The aim is to determine the critical micelle concentration of LA and GML to further correlate their micellar aggregation properties with the obtained morphological and fluidic properties of the phospholipid bilayers. 
The determination of the $\mathrm{CMC}$ value can be done by three different techniques: electrical conductivity, surface tension and the fluorescence probe ${ }^{26}$. The chosen technique was the fluorescence probe with fluorescence spectroscopy due to familiarity with the technique and equipment availability. The fluorescence probe works by monitoring the light absorbed by an indicator, named fluorophore, and the subsequent emission of this light of longer wavelength ${ }^{29}$. The difference between the exciting and emitted wavelengths is known as the Stokes shift ${ }^{30}$. The fluorescence spectrophotometer separates emitted light (long wavelength) from excitation light (short wavelength) by using a fluorophore with a large Stokes shift. In this particular case, 1-pyrenecarboxaldehyde was used as fluorophore because of its recognized reliability and agreement with the surface tension method to determine $\mathrm{CMC}^{31}$. Furthermore, its aldehyde group makes it polar and highly soluble in aqueous solvents in comparison with commonly used pyrene fluorophores ${ }^{32}$

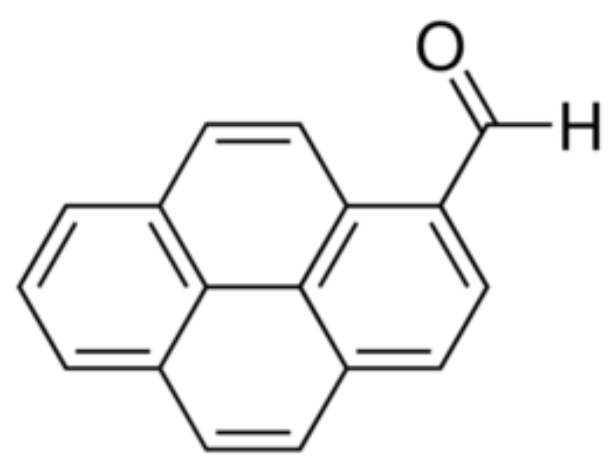

Figure 3.1 Chemical structure of 1-pyrenecarboxaldehyde. Fluorophore used to determine the $\mathrm{CMC}$ values in this thesis.

The fundament of the fluorescence probe with spectrometry is the detection of the concentration at which micelles start to form, evidence in the peak wavelength in the fluorescence spectrum. The peak wavelength is inversely proportional to micelle formation, indicating the interaction of the fluorophore with the hydrophobic long hydrocarbon-chains and hence, the decline in polarity and solubility. According to this, the highest concentration at which the first break of the peak occurs corresponds to the $\mathrm{CMC}^{31}$. In PBS buffer, it has been shown experimentally that the fluorescence emission spectrum 
of 1-pyrenecarboxaldehyde has a maximum peak wavelength at $473 \mathrm{~nm}$ and drops in the presence of micelles. Theoretically, this value was verified with the equation proposed by Ananthapadmanabhan et al. (Equation 3.1) which correlates polarity (dielectric constant of the solvent, D) with the position of the peak maximum ${ }^{31}$.

$$
\lambda_{\max }=0.52 D+431.5 \quad \text { Eq. } 3.1
$$

Water has a reported $\mathrm{D}$ of 80 , and based on the peak measurements of 1pyrenecarboxaldehyde in PBS $\left(\lambda_{\max }=473 \mathrm{~nm}\right)$ the result is in agreement with the literature ${ }^{33}$.

As a note, is worth to mention that that the CMC experimental results for LA and GML are encompassed in chapter 4. All of them performed in PBS buffer with the corresponding $\mathrm{pH}$ conditions to match the following QCM-D and fluorescence microscopy assays.

\section{Experimental Protocol:}

Initially, a stock solution of 1-pyrenecarboxaldehyde was prepared by dissolving the corresponding mass of compound in methanol to reach a final concentration of $5 \mathrm{mM}$ (stored at $4{ }^{\circ} \mathrm{C}$ ). The test samples were prepared by adding a small aliquot of the stock solution of fluorescent probe in methanol to a glass tube, and then the methanol was evaporated to form a dry thin film on the glass walls. Afterwards, the designated concentration of LA or GML in the appropriate solution was added to the vial, and the sample was vortexed followed by heating to $70{ }^{\circ} \mathrm{C}$ for 30 minutes. Finally, the sample was cooled down before measurements were conducted at room temperature. The initial concentration of the probe was $5 \mathrm{mM}$ and the final $0.1 \mu \mathrm{M}$. The CMC values of LA and GML in the appropriate $\mathrm{pH}$ conditions were done using a Cary Eclipse fluorescence spectrophotometer (Varian, Inc., Australia). In the measurements, the test samples were excited at $365.5 \mathrm{~nm}$ and the emission spectrum was recorded from 400 to $600 \mathrm{~nm}$. The highest-intensity wavelength in the emission spectrum was recorded for each measurement, and the mean and standard deviation are reported from six technical replicates. The first breakpoint of the curve represents the $\mathrm{CMC}$ value, concentration above 
which micelles are formed ${ }^{31}$. In the graph, the $\mathrm{CMC}$ value is defined as the highest test concentration before the peak wavelength starts to decrease.

\subsection{2. pH Titration method}

The $\mathrm{pH}$ titration method is "the quantitative addition of a solutions of known concentration to a solution of unknown concentration until the reaction between them is complete to determine the concentration of the second solution" 34 . Besides the determination of concentration, it is one of the oldest and more reliable methods to obtain the $\mathrm{pK}_{\mathrm{a}}$ value. It works by adding small aliquots of the titrant (acid or base) to the analyte (of opposite nature than the titrant). The change in $\mathrm{pH}$ is recorded with an indicator, litmus paper or $\mathrm{pH}$ meter. The point at which the volume of the titrant is chemically equivalent to the amount of the analyte is known as the equivalence point. With an indicator the reaction is judge to be completed when the analyte changes color, closely to the equivalence point. By means of a $\mathrm{pH}$ meter, as done for this study, the progress is monitored by plotting a titration curve, where the abscissa axis is the volume of the titrant and the ordinate axis the $\mathrm{pH}$. The inflexion point, center of the vertical region of the curve, is the equivalence point ${ }^{34}$ (Figure 3.2.). A substance can have multiple inflexion or equivalence points. The measurements were conducted at room temperature and the salt concentration was obtained by subtracting the value from the buffer (PBS). 


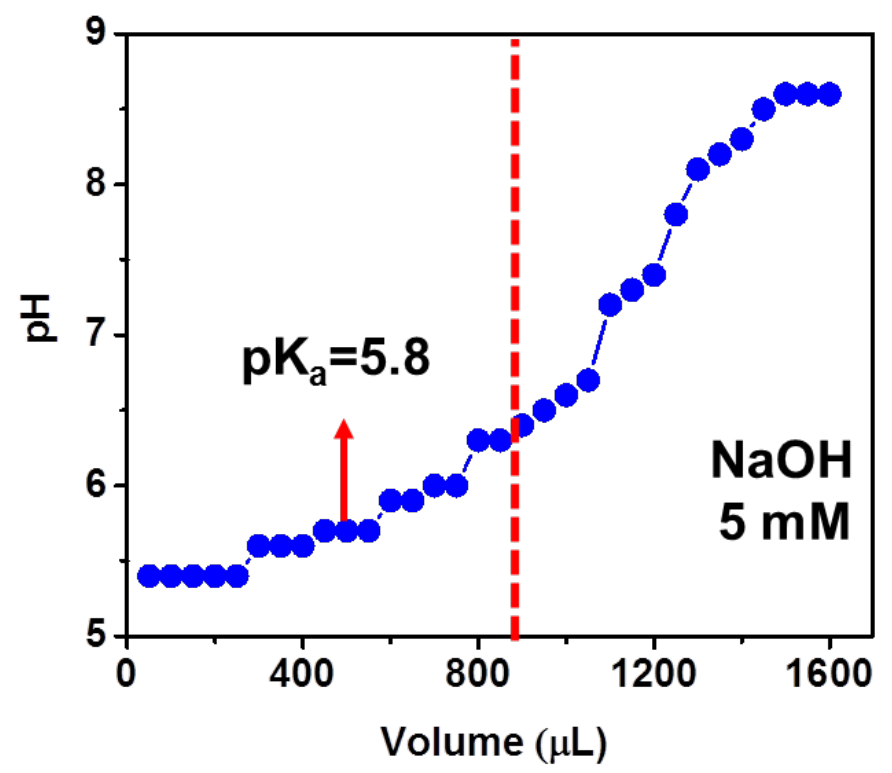

Figure 3.2. Graph of the equivalence point and $\mathrm{pK}_{\mathrm{a}}$ value of lauric acid in water. A titration curve was plotted by measuring the $\mathrm{pH}$ as a function of the volume of $\mathrm{NaOH}$. The equivalence point, center of the vertical region of the curve, was determined to be 7 (red dashed line) and the $\mathrm{pK}_{\mathrm{a}}$ value, half of the titration volume of $\mathrm{NaOH}$, was 5.8. The equivalence point is the median value of the curve.

Theoretically, the $\mathrm{pK}_{\mathrm{a}}$ values are calculated with the Henderson-Hasselbach equation (Eq. 3.2), where $[\mathrm{A}]$ is the concentration of conjugate base and $[\mathrm{HA}]$ is the concentration of the conjugate acid. ( $\mathrm{pH} 1 / 2$ is the $\mathrm{pH}$ value of half the volume of the titration) when $[\mathrm{A}]=[\mathrm{HA}]$. Accordingly, the $\mathrm{pK}_{\mathrm{a}}$ is the $\mathrm{pH}$ value corresponding to the half of the titration volume ${ }^{35}$

$$
\begin{gathered}
p H=p K_{a}+\log \left(\frac{[A]}{[H A]}\right) \quad \text { Eq. } 3.2 \\
p K_{a}=p H \frac{1}{2} \quad \text { Eq. } 3.3
\end{gathered}
$$

The $\mathrm{pH}$ titration method was employed to determine the $\mathrm{pK}_{\mathrm{a}}$ value of lauric acid with sodium hydroxide at room temperature. $\mathrm{NaOH}$ is a strong base and one of the simplest hydroxides, because of that it is typically used for titration. Overall, the documentation of the $\mathrm{pK}_{\mathrm{a}}$ value was important to correlate the results with the Critical Micelle Concentration (CMC), QCM-D data and minimum inhibitory concentration (MIC). When the $\mathrm{pH}$ of a 
molecule matches its $\mathrm{pK}_{\mathrm{a}}$ value half of it is protonated and half deprotonated, feature that will directly impact the mode of action.

\section{Experimental Protocol:}

A lauric acid solution in water $(5 \mathrm{mM}, 20 \mathrm{~mL})$ was titrated with a $0.5,2.5$ and $5 \mathrm{mM} \mathrm{NaOH}$ solution. The $\mathrm{NaOH}$ was added stepwise until the titration curve reached plateau. The change in $\mathrm{pH}$ of each solution was recorded using an Accumet $\mathrm{AB} 15 \mathrm{pH}$ meter (Thermo Fisher Scientific; Waltham, MA). The three results from the different molar concentrations were within the range of 5-6, and match with a negligible standard deviation.

\subsubsection{Quartz Crystal Microbalance-Dissipation}

The quartz crystal microbalance with dissipation (QCM-D) is a nanogram sensitive balance that measures the mass of soft or viscoelastic films. It works by recording the acoustic waves generated by an oscillating piezoelectric crystal quartz disc at a set up frequency ${ }^{36}$. The quartz crystal is located between two electrodes with applied alternating current, making it resonate at a frequency dependent on the material properties of the disc. The common disc is cut from bulk quartz using the "AT cut" to obtain the crystal move in antiparallel manner, have a resonance frequency of $5 \mathrm{MHz}$ and a thickness of $330 \mu \mathrm{m}{ }^{37}$. The first QCMs were developed to measure rigid thin films in vacuum chambers ${ }^{38}$; however, due to their expansion to other fields (biology, biotechnology, polymers and lipid studies) the need to assess films under wet conditions arised ${ }^{39}$. The new generation of QCM monitored not only the change in the frequency of oscillation $(\Delta \mathrm{f})$ related to the mass, but also the energy loss and viscoelasticity measured via dissipation change $(\Delta \mathrm{D})$, all in real time. QCM-D registers the shift in dissipation with the oscillation decay, reached by switching off the AC voltage applied to the electrodes, once the quartz crystal disc had been excited at its resonance frequency $(5 \mathrm{MHz}){ }^{40}$ (Figure 3.3.) 

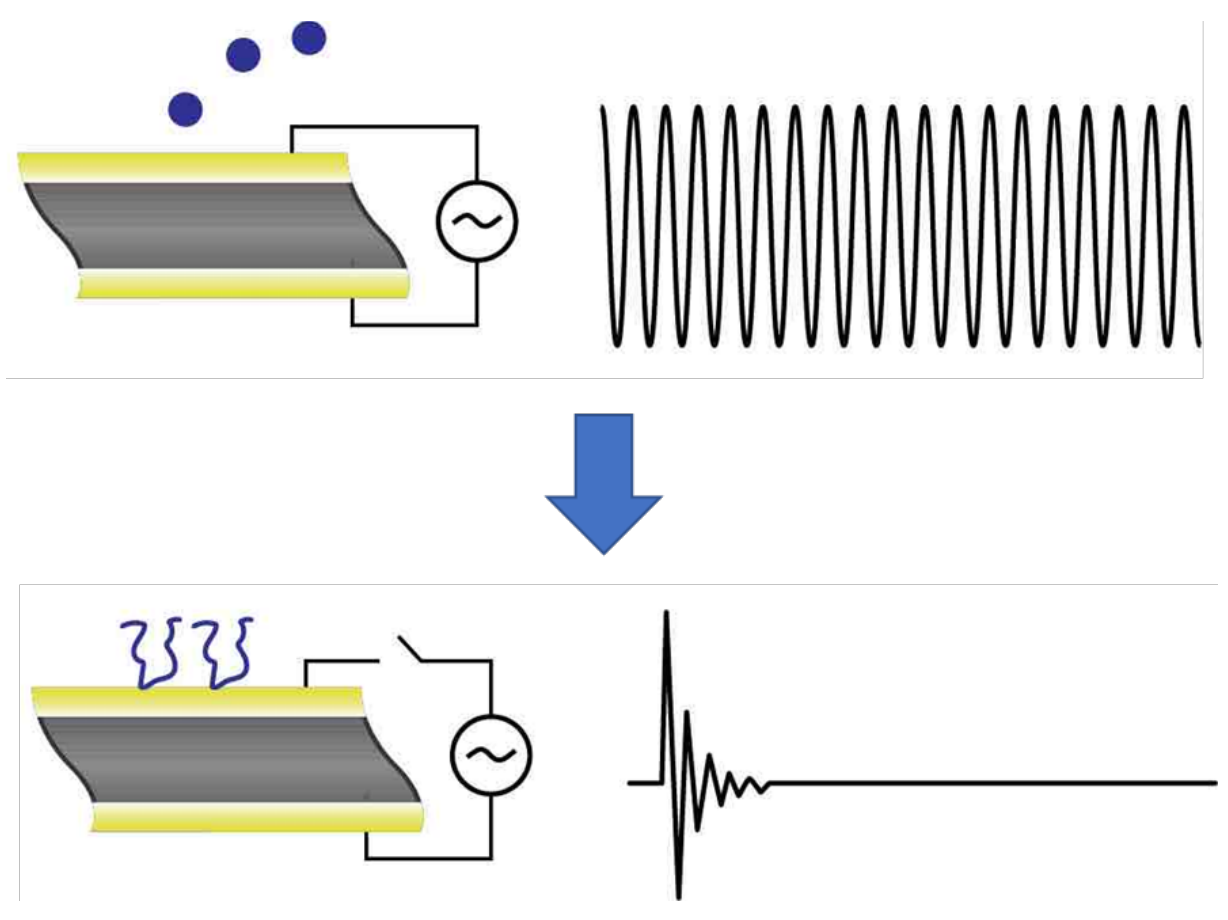

Figure 3.3. Diagram of the oscillation decay in QCM-D to measure the shift in dissipation. (A) The voltage is applied across the electrodes. (B) The oscillatory decay and pattern of a viscoelastic film when the quartz crystal disc comes to rest after switching off the voltage. Image modified from Biolin Scientific/Q-Sense.

In brief, the energy dissipation represents the sum of energy losses in the system and is a dimensionless value, as explained in the following equation ${ }^{41}$ :

$$
D=\frac{1}{Q}=\frac{E_{\text {dissipated }}}{2 \pi E_{\text {stored }}} \quad \text { Eq. } \mathbf{3 . 4}
$$

$Q$ represents the quality factor, $E_{\text {dissipated }}$ the energy dissipated during one oscillatory cycle and $E_{\text {stored }}$ the energy stored in the system while oscillating.

Regarding to the correlation with the decay and the resonance frequency, the QCM-D system utilizes the equation below (Eq. 3.5). Where $f$ is the frequency and $\tau$ the time constant for the decay ${ }^{40}$. 


$$
D=\frac{1}{\pi f \tau} \quad \text { Eq. } 3.5
$$

With an eye towards characterizing a membrane that resembles the phospholipid bilayer of the bacteria cell membrane, QCM-D was selected to investigate the interactions between antimicrobial lipids and supported lipid bilayers (SLB). This approach aided in the understanding of the mode of action of the amphiphilic compounds.

\section{Experimental Protocol:}

For the convenience of the experiments the SALB method was followed to fabricated the lipid bilayer over a silicon dioxide-coated sensor ${ }^{42}$. The method suggests the use of DOPC (1,2-dioleoyl-sn-glycero-3-phosphocholine) to fabricate the SLB due to the strong electrostatic interaction between the substrate, silicon dioxide, negatively charged and the positive lipid bilayer. This robust interaction between the bilayer and the substrate allows for a better coverage of the surface and a stable measurement of frequency and dissipation. The measurements were conducted with a four-channel Q-Sense E4 instrument (Biolin Scientific, Stockholm, Sweden), with simultaneous time-lapsed recording of shifts in the resonance frequency $(\Delta f)$ and energy dissipation $(\Delta D)$ of the oscillating, piezoelectric quartz crystal ${ }^{41}$. The sensor chips had a fundamental frequency of $5 \mathrm{MHz}$, and were coated with a sputter-coated, 50-nm-thick layer of silicon dioxide (model no. QSX 303, Biolin Scientific). Before experiment, the chips were sequentially rinsed with water and ethanol, dried with a stream of nitrogen gas, and treated with oxygen plasma for 1 min using an Expanded Plasma Cleaner (model no. PDC-002, Harrick Plasma, Ithaca, NY). After cleaning, the chips were immediately loaded into the measurement chambers and buffer solution was injected to establish a measurement baseline (10 mM Tris buffer, $150 \mathrm{mM}$ $\mathrm{NaCl}, \mathrm{pH}$ 7.5). Shortly after, isopropanol was injected for 10 mins for equilibration and then $0.5 \mathrm{mg} / \mathrm{ml}$ DOPC lipid in isopropanol was incorporated for the same time. Isopropanol was exchanged for Tris buffer and the quality of the SLB was controlled by measuring the optimal frequency and dissipation, $-26 \mathrm{~Hz}$ and $<0.3 \times 10^{6}$ respectively. Following the SLB fabrication, the Tris buffer was exchanged for PBS (the $\mathrm{pH}$ was adjusted according to the experiment conditions with $\mathrm{NaOH} 1 \mathrm{M}$ ). Lastly, the antimicrobial lipid (lauric acid or glycerol monolaurate) dissolved in PBS was injected at a flow rate of $50 \mu \mathrm{L} / \mathrm{min}$, as 
controlled by a peristaltic pump (Reglo Digital, Ismatec, Glattbrugg, Switzerland). In the particular case of the DOPC/GML bicelles, chapter 6, the bicellar samples were added under continuous flow conditions and after the lipid adlayers were formed, a buffer washing step was done over $10 \mathrm{~min}$. After complete washing step, whether SLB formation occurred or not was evaluated by reading the final $\Delta \mathrm{f}$ and $\Delta \mathrm{D}$ shifts. The temperature in the measurement chamber was maintained at $25.0 \pm 0.5^{\circ} \mathrm{C}$. The measurement data were recorded at the third $(n=3)$, fifth $(n=5)$, and seventh $(n=7)$ odd overtones using the QSoft software program (Biolin Scientific), and the data was normalized according to the overtone number. Data processing was performed in the QTools (Biolin Scientific) and OriginPro 8.5 (OriginLab, Northampton, MA) software programs. All presented data was collected at the fifth overtone.

\subsubsection{Fluorescence Microscopy}

Fluorescence microscopy is a key method in biology and material science. It allows the identification of specimens even when the structure is too small to be resolved in a light microscope $\mathrm{e}^{43}$. The concept of fluorescence has a foundation in the "Stoke shift" principle, widely describe in Section 3.4.1 (Critical Micelle Concentration Determination Assay). The principle states that the wavelength from the emitted light is always longer and thus lower in energy, than that of the excitation ${ }^{44}$. The separation of light beams is possible with the microscope emission filter, nonetheless, the modern epi-fluorescence microcopy assembles the main components of the microscope (excitation filter, dichroic mirror and emission filter) into a component known as filter cube. Within the several advantages of this illuminator the light source is positioned above the sample, in a vertical way, and the objective and condenser are put together as a single component for perfect alignment. The epi-fluorescence microscope works by directing the light coming from an arc lamp through the filter cube. The excitation wavelength passes through the objective and showers the sample. If it fluoresces, the emitted light passes again through the filter cube, which blocks the excitation wavelength. The filtered emission is sent to a detector that digitalize the image to be visualized on a screen (Figure 3.4.) ${ }^{44-46}$. Is worth to highlight, that the indicator 
or fluorophore is as equally important as the microscope, since it will determine the excitation and emission wavelength.

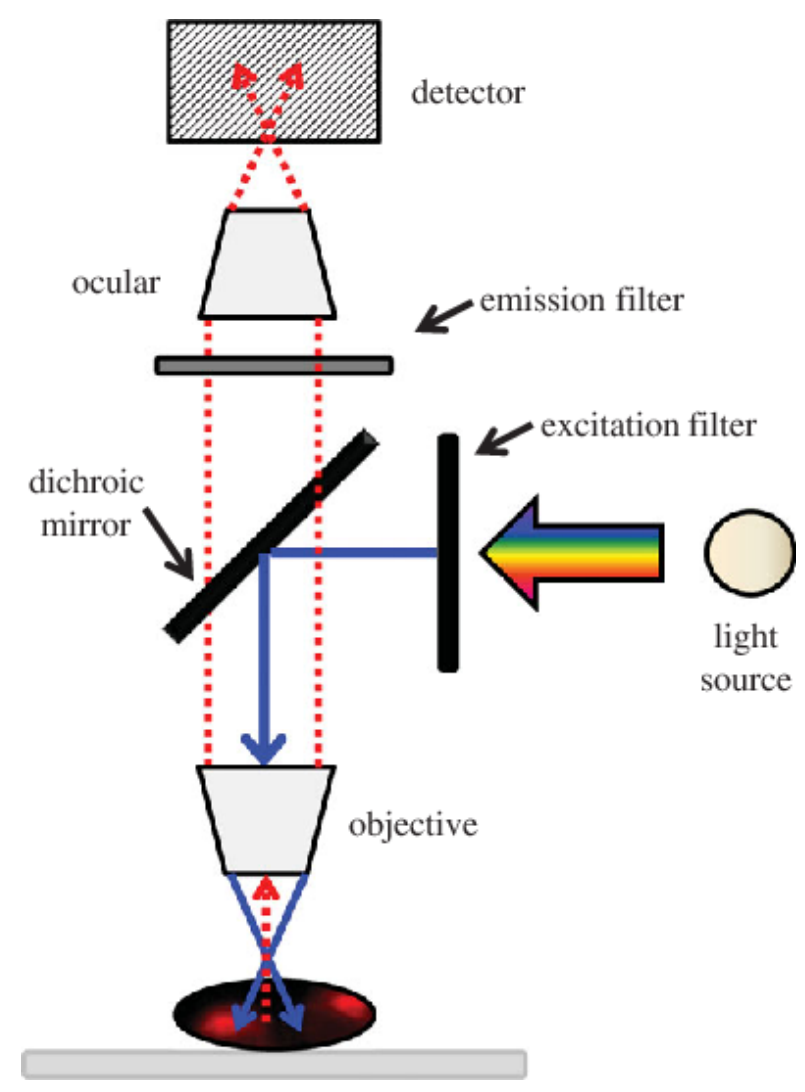

Figure 3.4. Schematic diagram of the main components of an epi-fluorescence microscope.

Apropos this thesis, the epifluorescence microscopy experiments were conducted to directly observe morphological changes in SLBs on glass surfaces upon treatment with LA or GML; and for the DOPC/GML bicelles in chapter 6. The chosen fluorophore, Liss Rhod$\mathrm{PE}$, labels the phospholipid heads giving bright-red fluorescent conjugates ${ }^{47}$. Liss RhodPE was blended with the phospholipids at the moment of fabricating the supported lipid bilayer.

\section{Experimental Protocol:}

The epi-fluorescence microscopy experiments were conducted using an Eclipse TI-U inverted optical microscope (Nikon, Japan) with a $60 \times$ magnification $(\mathrm{NA}=1.49)$ oil- 
immersion objective lens (Nikon), and images were collected with an iXon 512 pixel $\times 512$ pixel EMCCD camera (Andor Technology, Northern Ireland). The pixel size was $0.267 \times$ $0.267 \mu^{2}$. A fiber-coupled mercury lamp (Intensilight C-HGFIE, Nikon) was used to illuminate fluorescently labeled phospholipids (a small fraction of all phospholipids in the SLB) with a TRITC filter. For the experiments, the SLB was fabricated following the vesicle fusion method with DOPC, and $0.5 \mathrm{~mol} \%$ of the fluorophore, 1,2-dipalmitoyl-snglycero-3- phosphoethanolamine-N-(lissamine rhodamine B sulfonyl) lipid. The vesicles were prepared with Tris buffer $(150 \mathrm{mM} \mathrm{NaCl}, \mathrm{pH} 7.5)$ and extruded through $50 \mathrm{~nm}$ diameter polycarbonate membranes (Whatman Schleicher \& Schuell, Dassel, Germany). The resulting vesicles had an average diameter of $60 \mathrm{~nm}$ and the solution a concentration of $0.2 \mathrm{mg} / \mathrm{ml}$. SLBs were formed on a glass slide that was enclosed within a microfluidic flow-through chamber (sticky slide VI 0.4, Ibidi, Germany). After SLB formation, the measurement chamber was rinsed with PBS buffer solution, and then the test compound was added under continuous flow conditions at a flow rate of $40 \mu \mathrm{L} / \mathrm{min}$. Time-lapse micrographs were recorded every $5 \mathrm{~s}$ for a total duration of $30 \mathrm{~min}$, and the initial time, $t=$ $0 \mathrm{~s}$, was defined by when the test compound solution reached the measurement chamber. The fluorescence intensity profile of each micrograph was normalized using a customwritten script for the Python(x,y) 2.7.5 software program.

\subsubsection{Minimum Inhibitory Concentration (MIC) Testing}

The definition of minimum inhibitory concentration is the lowest concentration of an antimicrobial agent that prevents visible growth of a microorganism under defined conditions $^{48}$. The MIC method is a valuable parameter to test antibiotic susceptibility, survey antibiotic resistance, compare antimicrobial agents or bacteria, and of great importance, to evaluate new drugs. It is indeed, one of the first steps in drug development to gauge the efficacy and growth inhibition (bacteriostatic activity) of bacteria ${ }^{48}$. Followed by the determination of the minimum bactericidal concentration (MBC), further explained in the next section. Returning to assessment of the bacteriostatic concentrations, the most common employed techniques are agar and broth dilution. Both methods have been standardized to comply with the reproducibility norms dictated by the two established 
committees, the CLSI (Clinical and Laboratory Standards Institute) ${ }^{49}$ and the EUCAST (European Committee for Antimicrobial Susceptibility Testing) ${ }^{50}$. In accordance with them, the agar method is appropriate to evaluate a small range of bacteria species when a large amount of antibacterial compounds are available. The broth method is better for the assessment of a significant number of bacteria species or compounds. The tests in this thesis were done with the "broth microdilution method" with slightly modifications to ensure the chemical stability and solubility of the antimicrobial lipids, LA and GML. Always following the guidelines for both tested anaerobic, C. acnes, and aerobic, S. aureus, bacteria $^{49,51}$. Regarding to the culture medium, the recommended standard ones were also employed. For C. acnes, Tryptic soy broth (TSB) and for S. aureus Mueller-Hinton (MH) broth.

The protocol for the broth method implies the twofold serial dilution of the antibacterial compound in growth medium. Next, the dilutions are inoculated with a fixed number of bacteria, incubated over a period of time and the optical turbidity of each well is recorded (Figure 3.5.). Depending on the total volume per well of the sample it is termed macro (2 $\mathrm{ml})$ or micro $(\leq 500 \mu \mathrm{l})$ dilution ${ }^{50}$. The modifications done to the original protocol were the incubation of the bacteria in buffer, specifically PBS, instead of growth medium; the streaking of the incubated samples on agar plates in lieu of the turbidity measurement; and the incubation for 1 hour rather than 3 . The justification behind these adjustments are the use of the same media/buffer, PBS, in the biophysical assays to warranty a concentrationdependent effect over the antimicrobial lipids. The streaking over agar plates eased the growth of bacteria, which would be slowed down with the PBS. The incubation time, only for $C$. acnes was limited to 1 hour. The standard conditions dictate an incubation time of lipids against bacterial cell membrane of 5 hours in average ${ }^{11,52}$, nevertheless, the aim was to assess merely the effect of the antimicrobial lipids and organic acid without the low $\mathrm{pH}$ playing a major role in the obtained outcome. As seen in other publications, C. acnes has a limited or null growth under acidic conditions ${ }^{53}$, so this was an amendment to the published pH-related studies and assisted in the direct correlation with the biophysical methods. 


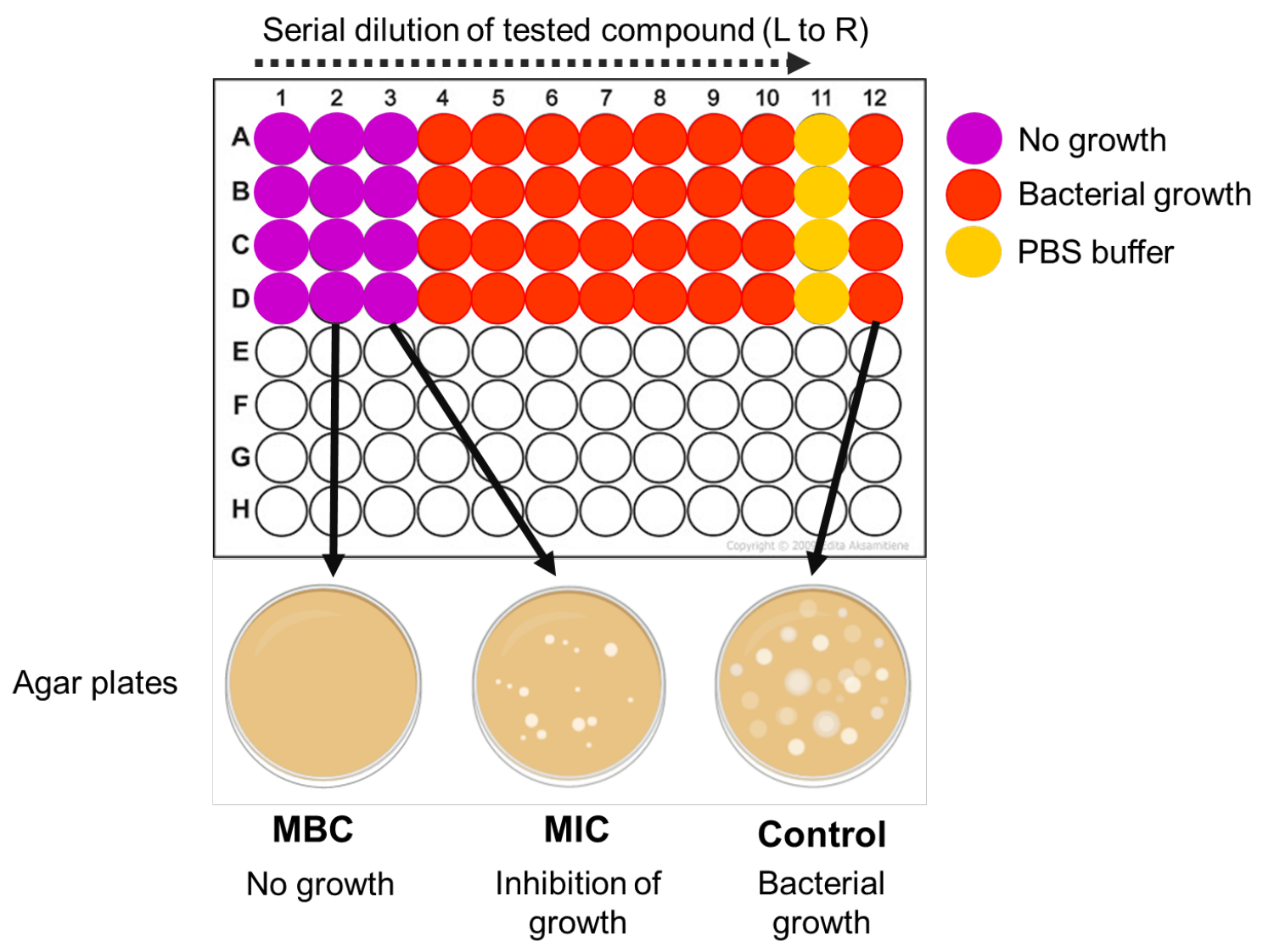

Figure 3.5. Schematic representation of a plate tested for MIC and MBC after incubation.

After incubation under anaerobic or aerobic conditions the samples were spotted onto agar plates and reincubated for the final assessment of bacterial occurrence. The minimum inhibitory concentration (MIC) was determined as the lowest amount of antibacterial agent that exerted a bacteriostatic behavior. The minimum bactericidal concentration was defined as the lowest amount of antibacterial agent killing $99.99 \%$ of bacteria. All experiments were accompanied by their Positive (bacteria in buffer) and Negative (buffer alone) controls to minimize the effect of other variables.

\section{Experimental Protocol:}

\section{Cutibacterium acnes}

The MIC values of antimicrobial lipids and glycolic acid against $C$. acnes were conducted for chapters 4 and 5. The test compounds were initially prepared with PBS at the corresponding $\mathrm{pH}$ value and diluted in a twofold dilution series in a 96-well plate. An equivalent volume of $C$. acnes at a concentration of $2 \times 10^{6} \mathrm{CFU} / \mathrm{mL}$ was added to each well, yielding a final cell density of $1 \times 10^{6} \mathrm{CFU} / \mathrm{mL}$. The plates were cultured for $1 \mathrm{hr}$ 
under anaerobic conditions at $37^{\circ} \mathrm{C}$, spotted on Tryptic soy agar plates, supplemented with $5 \%$ defibrinated sheep blood. The streaked plates were incubated under anaerobic conditions at $37{ }^{\circ} \mathrm{C}$ for 4 days, after which the presence of bacterial colonies in each test group was evaluated. The MIC values were determined by identifying the lowest concentration of test compound that completely inhibited the visible growth of bacterial colonies.

\section{Staphylococcus aureus}

The inhibitory activities of the DOPC/GML bicelles were determined in chapter 6. Initially, a two-fold dilution series from the q-ratio 0.25 was made in a 96 -well plate. Then, an equal volume of $S$. aureus was added to each dilution to reach a final cell density of $5 \times 10^{5} \mathrm{CFU}$ $\mathrm{mL}^{-1}$. The samples were incubated for $3 \mathrm{hr}$ at $37^{\circ} \mathrm{C}$ and spotted onto $\mathrm{MH}$ agar plates. The plates were incubated overnight at $37^{\circ} \mathrm{C}$ and the MIC values were assessed according to the occurrence of bacterial colonies.

\subsubsection{Minimum Bactericidal Concentration (MBC) Testing}

The minimum bactericidal concentration (MBC) is defined as the lowest concentration of drug required to kill most ( $\geq 99.9 \%$ ) of the final bacteria inoculum after incubation for a fixed length of time under set up conditions ${ }^{54}$. The knowledge of the MBC value, as with the MIC, aids in the continuous assessment of bacterial resistance and the development of new drugs ${ }^{55}$. The lethal concentration, minimum lethal concentration (MLC), as it is also known, has been standardize by the CLSI (Clinical and Laboratory Standards Institute) to ensure the reproducibility and reliability inter-laboratories and inter-technicians. The procedure to determine the MBC involves the broth macro $(2 \mathrm{ml})$ or micro $(\leq 500 \mu \mathrm{l})$ dilution, twofold dilutions of the test antibacterial agent in culture media, with the respective inoculum. Incubation under anaerobic or aerobic conditions at $37{ }^{\circ} \mathrm{C}$ for an specific set of time. It should be stress that until this point the MIC protocol is identically obeyed. The samples from the tubes or wells are sub-culture on agar plates and once again incubated under anaerobic or aerobic conditions for the specified timing. The lethal value is the lowest concentration of antibacterial agent that decreases the CFU (colony-forming 
unit) by $99.9 \% .{ }^{56}$ (Figure 3.5.). Regarding to the culture media, the CLSI, as with MIC, recommends the use of Tryptic soy broth for $C$. acnes and Mueller-Hinton broth for $S$. aureus. The MBC protocol in this investigation was followed as described above, with exception of the culture media in the microdilutions, which was exchanged for PBS buffer (1X, $\mathrm{pH} 7.4)$ to match the outcomes with the biophysical assays.

\section{Experimental Protocol:}

\section{Cutibacterium acnes}

Glycolic acid samples were prepared at different $\mathrm{pH}$ values in the concentration range of $200 \mathrm{mM}$ to $1.6 \mathrm{mM}$ and incubated with $C$. acnes at a cell density of $1 \times 10^{6} \mathrm{CFU} \mathrm{mL}^{-1}$. The samples were cultured under anaerobic conditions for $1 \mathrm{hr}$ at $37^{\circ} \mathrm{C}$, and then each sample was diluted in a 10-fold series. A $100 \mu$ volume of each diluted was streaked onto tryptic soy agar plates supplemented with 5\% defibrinated sheep blood. The streaked plates were incubated under anaerobic conditions at $37^{\circ} \mathrm{C}$ for 4 days, and the $\mathrm{CFU}$ density of each sample was next determined. The effect of glycolic acid was resolute with the MBC value, expressed as the lowest concentration of glycolic acid that reduced bacterial cell viability by at least $99.99 \%$. The obtained data is included in chapter 5 .

\section{Staphylococcus aureus}

The DOPC/GML bicelles q-ratio 0.25 ranging from $0.031 \mathrm{mM}$ to $2 \mathrm{mM}$ (GML concentration) was cultured with $S$. aureus $\left(5 \times 10^{5} \mathrm{CFU} \mathrm{mL} \mathrm{mL}^{-1}\right)$ and incubated for $3 \mathrm{hr}$ at $37^{\circ} \mathrm{C}$. Afterwards, the samples were diluted in a 10-fold series, streaked onto $\mathrm{MH}$ agar plates $(10 \mu \mathrm{l})$ and incubated overnight at $37^{\circ} \mathrm{C}$. The $\mathrm{MBC}$ value was defined as the lowest DOPC/GML bicelle concentration that diminished $99.99 \%$ of the bacterial cell count. The outcomes for this assay are found in chapter 6 .

\subsubsection{Live/Dead Bacterial Cell Staining}

The bacterial death has normally been assessed by the absence of visible growth on agar plates $^{57}$. Traditional viability assays are based on culture methods and involve metabolic characteristics or membrane integrity. Unfortunately, these methods are very sensitive to 
growth $^{58}$ and cannot differentiate between dead and injured cells. The membrane integrity technique presents high levels of background interference ${ }^{59}$ and the metabolic characteristic assay only works with certain types of bacteria ${ }^{60}$. Novel methods, utilize fluorescence indicators with the capacity to target single cells by using fluorescence microscopy, flow cytometry or fluorometer. The LIVE/DEAD viability kit (BacLight Bacterial Viability Kit, Molecular Probes, Invitrogen) is the most often used because of its reliability and practicality to distinguish live and dead microorganisms within minutes. It is a direct-count assay for a diverse array of bacteria genera under a variety of environmental conditions. The kit consist of two nucleic acid dyes, SYTO9 and propidium iodide (PI). SYTO9 penetrates all cells, intact or damage, and stains them in green. Contrarily, propidium iodide dyes in red just the bacteria with damaged membranes. The excitation and emission wavelengths for the stains are 480/500 nm for SYTO9 and 490/635 nm for $\mathrm{PI}^{61}$.

The confocal laser scanning microscopy (CLSM) technique was chosen to visualize the LIVE/DEAD bacteria. The samples, C. acnes treated with glycolic acid and S. aureus with glycerol monolaurate bicellar system, were labeled with the stains from the viability kit to confirm the killing effect of the compounds (Figure 3.6.).

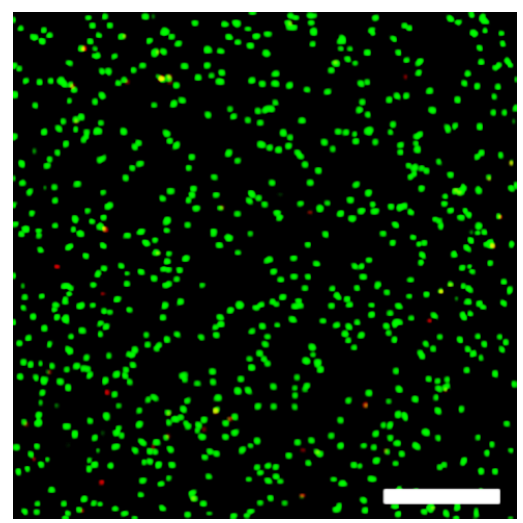

Positive Control

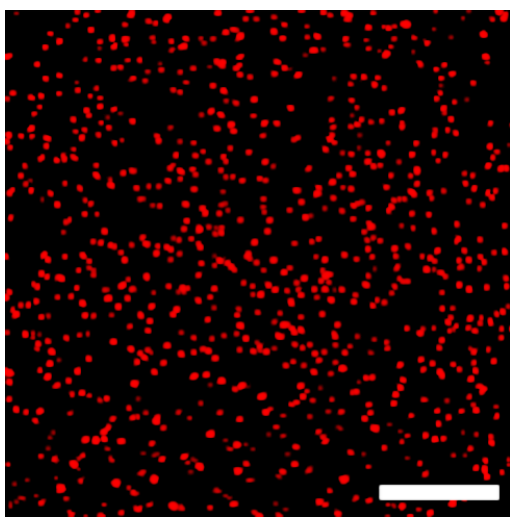

Negative Control

Figure 3.6. Example of images taken with CLSM using the Live/Dead BacLight Bacterial Viability Kit with $\boldsymbol{C}$. acnes bacteria. Live bacteria cells are dyed in green (Left panel) while dead in red (right panel). The positive control is C. acnes in PBS and the negative in ethanol $90 \%$. Bacterial concentration of $1 \times 10^{8} \mathrm{CFU} \mathrm{mL}^{-1}$. Scale bar of $100 \mu \mathrm{m}$. 
The confocal laser microscope works under the same concept of fluorescence excitation and emission coined by Stokes. A fluorophore absorbs light (short wavelength) and emits it back with less energy (long wavelength). This shift in wavelengths size is detected by the microscope and digitalized as an image ${ }^{62}$. The advantage that confocal microscopy offers over other techniques is the elimination of out of-focus blur, which leads to the capture of 3D-like fluorescence images of thick and transparent specimens ${ }^{63}$. Furthermore, the confocal microscope bridged the gap between wide-field light and transmission electron microscopy. The CLSM has better resolution than a light microscope (maximum resolution $0.2 \mu \mathrm{m})$, but not as good as that from a transmission electron microscope $(0.1$ $\mathrm{nm})^{64}$. The operation principle relies on the pinhole, adjustable iris, which rejects the out of focus light from the image. The excitation source, a laser light beam, is focused on the sample by reflection from the dichroic mirror and the objective lens. The sample is sequentially scanned by the laser and the emitted fluorescence passes again through the dichroic mirror, and is focused onto the image plane. The pinhole only allows the emitted light from the confocal plane of the sample to reach the detector (photomultiplier tube) ${ }^{45}$ (Figure 3.7.). The formation of the image is achieved by the production of optical sections from the laser scans across the sample. This non-invasive sectioning makes it ideal for living samples ${ }^{64}$. 


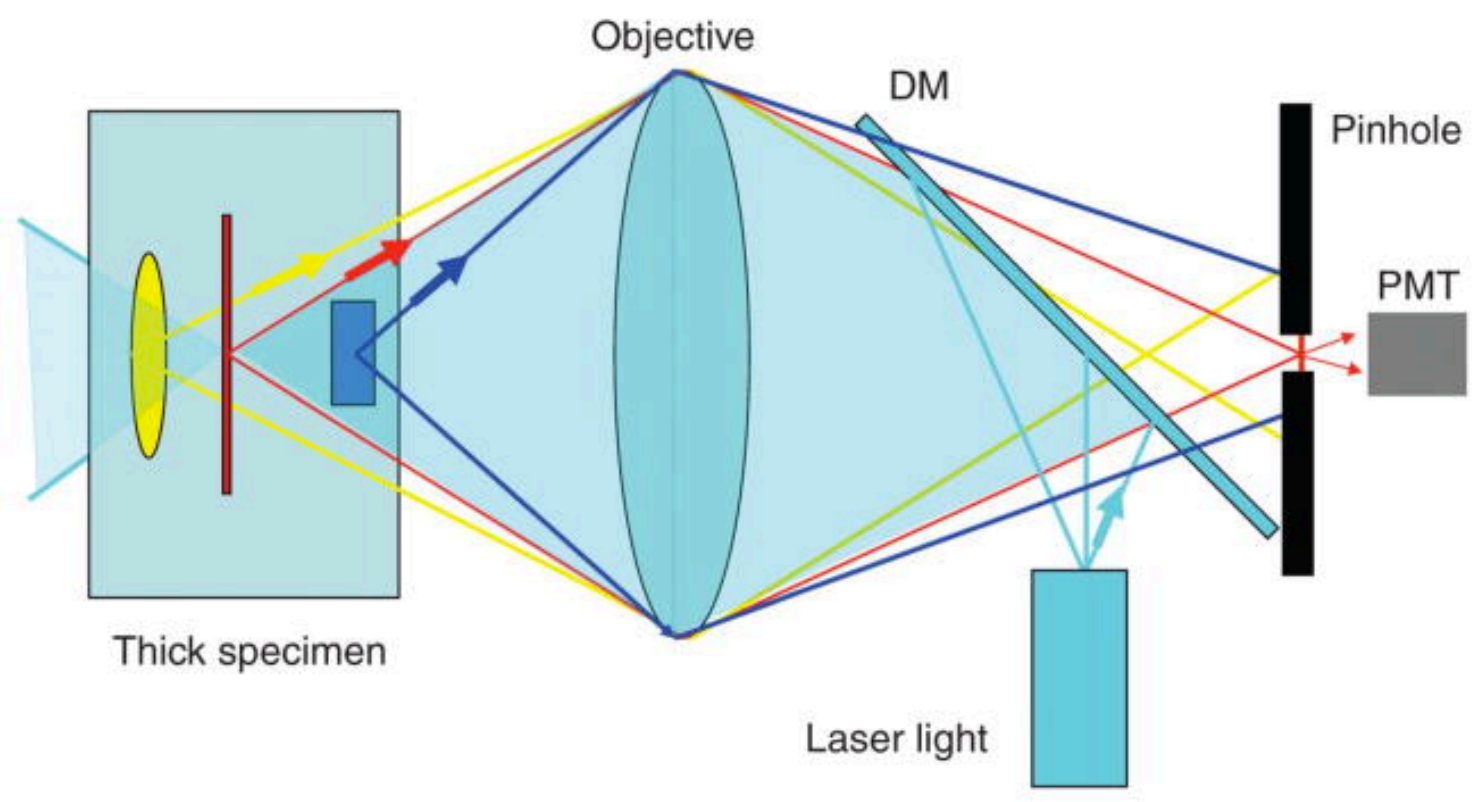

Figure 3.7. Schematic representation of the confocal laser scanning microscope (CLSM) operation principle. The adjustable iris, pinhole, only allows light from the confocal plane of the specimen to reach the photomultiplier. Image retrieved from Sanderson et al. ${ }^{45}$.

\section{Experimental Protocol:}

\section{Cutibacterium acnes}

Glycolic acid samples in chapter 5, were prepared at pH 3.5 in the concentration range of $50 \mathrm{mM}$ to $3.1 \mathrm{mM}$ and then added to $C$. acnes suspensions at a final concentration of $1 \times$ $10^{8} \mathrm{CFU} \mathrm{mL} \mathrm{m}^{-1}$. The samples were incubated under anaerobic conditions for $1 \mathrm{hr}$ at $37^{\circ} \mathrm{C}$ and then stained with fluorescent dyes by using the Live/Dead BacLight Bacterial Viability Kit (Molecular Probes, Invitrogen, Carlsbad, CA). The dye mixture was prepared by combining equal volumes of the reagents, SYTO9 and PI, in a microfuge tube. A volume of $3 \mu \mathrm{l}$ of the combined reagent mixture was added for each milliliter of bacterial suspension. It was thoroughly mixed and incubated at room temperature in the dark for 15 mins. Without delay, the stained bacterial samples were trapped between a slide and a coverslip to be observed using an LSM 710 confocal laser scanning microscope (Zeiss, Oberkochen, Germany). 


\section{Staphylococcus aureus}

In chapter 6 , the DOPC/GML bicelles with a q-ratio of 0.25 were prepared within a GML concentration of $0.031 \mathrm{mM}$ to $1 \mathrm{mM}$. Shortly after, added to $S$. aureus suspensions at a final concentration of $1 \times 10^{7} \mathrm{CFU} \mathrm{mL}^{-1}$. The samples were incubated for $3 \mathrm{hr}$ at $37^{\circ} \mathrm{C}$ and then stained with the dyes from the Live/Dead BacLight Bacterial Viability Kit (Molecular Probes, Invitrogen, Carlsbad, CA). The dye mixture was prepared by combining 1:1.5 volumes of the reagents, SYTO9:PI, in a microfuge tube. A volume of $3 \mu$ of the combined reagent mixture was added for each milliliter of bacterial suspension. It was thoroughly mixed and incubated at room temperature in the dark for 15 mins. Without delay, the stained bacterial samples were trapped between a slide and a coverslip to be observed using an LSM 710 confocal laser scanning microscope (Zeiss, Oberkochen, Germany).

\subsubsection{ATP Bioluminescence assay}

The ATP bioluminescence assay is an in-vitro and in-situ evaluation method for cell proliferation. It quantitatively measures adenosine triphosphate (ATP) produced by bacteria and fungi ${ }^{56}$. ATP is the energy source in all living cells and is stored in the mitochondria and cytoplasm in steady millimolar concentrations ${ }^{65,66}$. Hence, its measurement functions as a marker of bacteriolytic activity, triggered by membrane disruption and the release of all cytolytic free $\mathrm{ATP}^{67,68}$. On the other side, bioluminescence, is the production and emission of light by living organisms after an enzymatic reaction ${ }^{69}$. The reaction is sparked by the firefly enzyme luciferase, which causes the oxidation of the substrate luciferin. The product is a photon of light, easily quantify with a luminometer. Currently, assays based on bioluminescence are the best to measure intracellular and extracellular $\mathrm{ATP}^{66}$. The main advantages of the methods are high sensitivity, in the range of micro and pico molar levels, speed and accuracy for real time viability survey ${ }^{67,70}$. They have a wide range of application for cell proliferation, cytotoxicity, antimicrobial activity and viability ${ }^{56}$.

The occupied kit for the assessment of bacterial membrane damage was ATP Bioluminescence Assay Kit HS II (Roche, Mannheim, Germany). The test principle is the 
catalyzed reaction of luciferin into oxyluciferin in the presence of ATP and $\mathrm{O}_{2}$ with the reagent luciferase ${ }^{71}$ (Eq. 3.6)

$$
A T P+D \text { luciferin }+O_{2}=\text { oxyluciferin }+A M P+P P_{i}+\text { light } \quad \text { Eq.3.6 }
$$

AMP $=$ Adenosine monophosphate

PPi $=$ Pyrophosphate

The product of the enzymatic reaction is green light with a maximum emission of $562 \mathrm{~nm}$. In essence, the emitted light is proportional to the ATP concentration and therefore directly proportional to the number of living/death cells ${ }^{71,72}$. The readouts were conducted with a multi-mode microplate reader, Cytation 5 (BioTek, Winooski, VT) and the software Gen 5. Luminescence detection, unlike fluorescence, does not require a light lamp or an objective for the selection of excitation wavelengths. The optical system consist of a lighttight reading chamber and the detector (photomultiplier tube) ${ }^{73}$. The rest depend on the luciferase enzyme and the reaction.
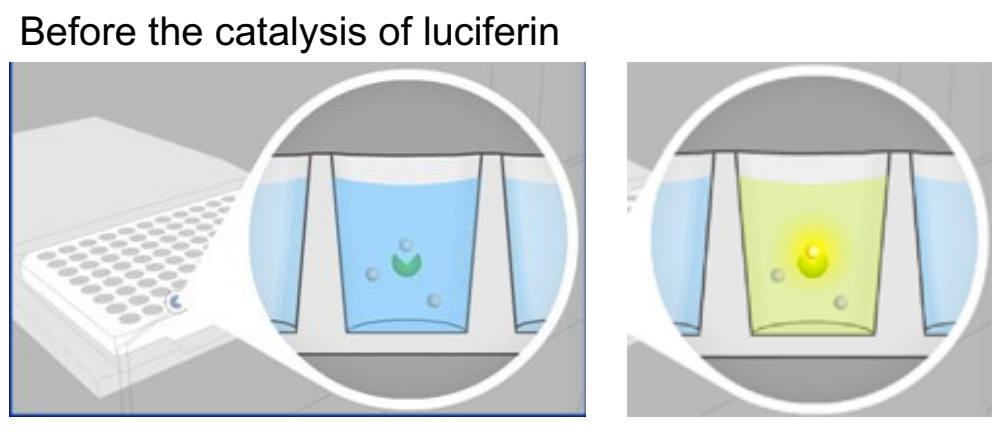

The enzymatic reaction produces green light

Figure 3.8. Illustration of the enzymatic reaction of luciferin in a microtiter plate. The reagent luciferase was added to the samples in a white microtiter plate. Immediately after, the reaction produces green light, proportional to the amount of ATP in the media. Retrieved from BioTek Glow Luminescence $^{73}$. 
The bioluminescent readouts were converted into ATP concentrations based on a standard curvature, made with serial dilutions of the ATP standard reagent in the sample buffer, PBS (Figure 3.9).

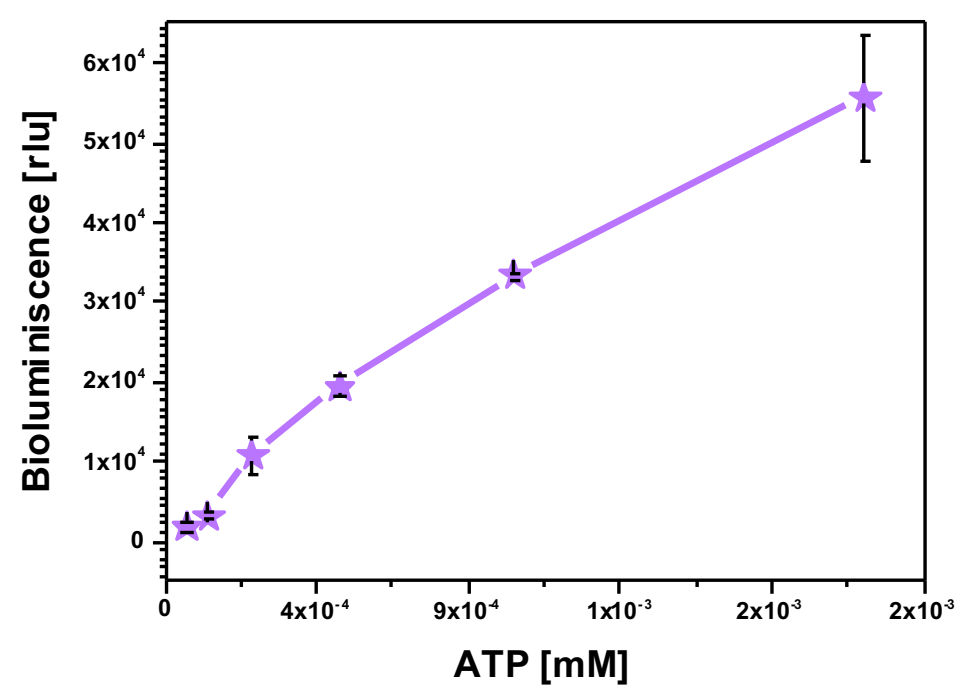

Figure 3.9. ATP Standard Curve. The ATP standard reagent from the Bioluminescence Assay Kit HS II was diluted in series in a white microtiter plate. The luciferase reagent was added to the diluents and immediately after bioluminescence was measured with the multi-mode microplate reader, Cytation 5. The data was plotted in a log-log curve of ATP concentration (mM) against Bioluminescence relative light units (rlu). Each point in the graph represents the mean value from 3 technical replicates and their standard deviation.

\section{Experimental Protocol:}

C. acnes suspension was diluted to an $\mathrm{OD}_{600}$ value of $\sim 0.5$ that corresponds to a density of

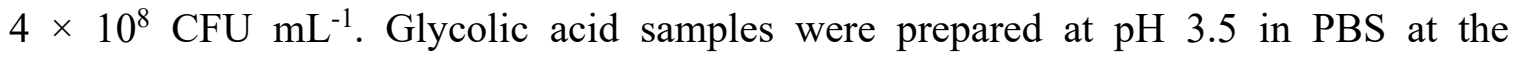
concentration range of $50 \mathrm{mM}$ to $12.5 \mathrm{mM}$ and then added to C. acnes suspension at a final concentration of $2 \times 10^{8} \mathrm{CFU} \mathrm{mL}^{-1}$. The samples were prepared in a white microtiter plate to avoid cross talk between individual wells, and further incubated under anaerobic conditions for $1 \mathrm{hr}$ at $37^{\circ} \mathrm{C}$. After incubation, the samples were titrated with $3 \mathrm{M} \mathrm{NaOH}$ to increase the $\mathrm{pH}$ value to $\sim 7.5$, which is suitable for bioluminescence measurements. The samples were analyzed by the ATP Bioluminescence Assay Kit HS II (Roche, Mannheim, 
Germany), by adding an equal volume of the luciferase reagent to each well. Immediately after the relative amount of bioluminescence was recorded by using a Cytation 5 cell imaging multi-mode microplate reader (BioTek, Winooski, VT). Positive and negative controls were included and prepared by boiling $C$. acnes at a cell density of $2 \times 10^{8} \mathrm{CFU}$ $\mathrm{mL}^{-1}$ for 10 mins, and PBS $\mathrm{pH} 7.4$, respectively. The bioluminescent signals were converted into ATP concentrations based on the standard curvature, made with dilutions from the ATP standard reagent included in the kit. All results from this assay are encompassed in chapter 5.

\subsubsection{Dynamic Light Scattering (DLS)}

Dynamic light scattering is a well-established, standardized technique for particle size analysis and distribution. It is a non-invasive technique that requires small amounts of sample. DLS provides reliable and fast approximations of particle size within the nano and micro scale. On top of that, it is possible to analyze numerous solvents and concentrations in a wide array of temperatures. The applications for DLS are many, notwithstanding, among the most prominent are the study of proteins and polymers ${ }^{74,75}$.

The cornerstone of DLS is the Brownian motion principle. Particles dispersed in a liquid solvent are constantly moving and colliding without a pattern. Smaller particles move faster than larger ones due to the greater effect of the collision energy. By simply gauging the speed of the particles it is possible to determine the hydrodynamic radius using the StokesEinstein equation (Eq. 3.7). In the equation, the particle speed is denoted by the translational diffusion coefficient $(Ð)$. The temperature $(T)$ and viscosity $(\eta)$ are the key parameters affecting the particle motion $^{76}$.

$$
Đ=\frac{k_{B} T}{6 \pi \eta R_{H}} \quad \text { Eq. } 3.7
$$

$k_{B}=$ Boltzmann constant

$R_{H}=$ Hydrodynamic radius 
As described by the equation, the particle size is calculated according to the particle motion, not the direct measurement. The hydrodynamic radius is defined as "the radius of a hypothetical sphere that diffuses at the same rate as the particle under investigation"77. The terminology refers to the apparent size of the particle and might differ from the value obtained with other techniques which gauge physical parameters ${ }^{78}$.

DLS also examines the particle size distribution, given by the polydispersity index (DI). The term polydispersity or dispersity, as lately amended by the IUPAC, is utilized to describe the degree of non-uniformity of size distribution of particles ${ }^{79,80}$. An homogeneous samples, with particles of the same size (monodisperse) would have a DI of 0.00 , whereas an heterogenous sample, with particles of multiple size (entirely polydisperse) would be in the range of $1^{81}$.

The basic operational system of DLS consist of a laser that strikes light onto a sample contained in a cuvette. The particles in the sample cause the scattering of light in all directions. The detector captures this signal at a fixed angle and calculates the diffusion coefficient and the particle size by means of the Stokes-Einstein equation ${ }^{82}$ (Figure 3.10.). The arrange of the detection angle depends on the turbidity of the sample. A $15^{\circ}$ angle is generally used to monitor aggregations, $90^{\circ}$ for weakly scattering small particles and $175^{\circ}$ for highly concentrated samples ${ }^{76,82}$.
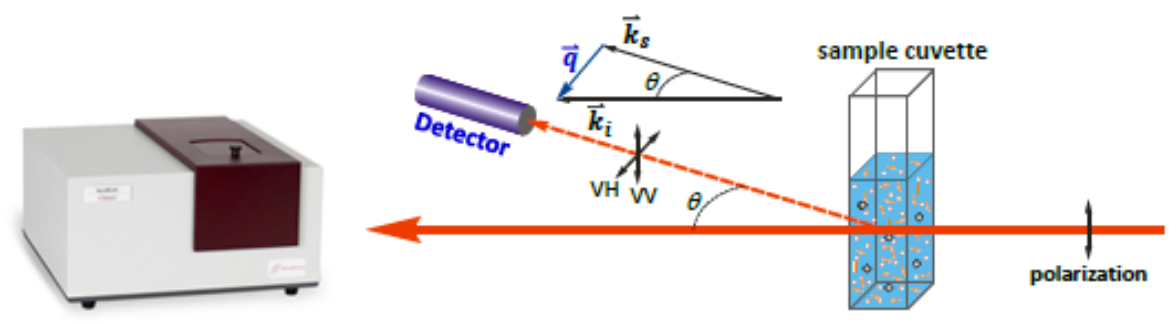

Figure 3.10. Basic setup of DLS measurement system with a particle size analyzer. The laser light is focused over the sample and the scattered light can be detected in several angles. Retrieved from Brookhaven Instruments ${ }^{83}$. 


\section{Experimental Protocol:}

For chapter 6, the average hydrodynamic diameter and dispersity index of the bicelles were analyzed by means of DLS with a particle size analyzer (ZetaPALS, Brookhaven Instruments, Holtsville, NY, USA). The instrument is equipped with a $658.0 \mathrm{~nm}$ monochromatic laser. Measurements were performed with a side scattering angle, $90^{\circ}$, at room temperature. A volume of $200 \mu \mathrm{L}$ of each sample was poured in a spectrophotometric polystyrene cuvette (spectral range 340-800 nm) and measured. The data acquisition and correlation of the intensity signal with the size distribution was done using the BIC Particle Sizing software (v5.27; Brookhaven Instruments). All reported values were obtained from five technical replicates.

\subsubsection{Cryo-Transmission Electron Microscopy (TEM)}

Cryo transmission electron microscopy is a technique used to image radiation-sensitive specimens under cryogenic conditions (below $\left.-150^{\circ} \mathrm{C}\right)^{84}$. The technique was initially developed to resolve images of proteins, virus and cell organelles that were impossible to observe under the light microscope ${ }^{85}$. In principle, the operational system of a TEM is analogous to a light microscope, the prime difference is the electron radiation source. Electrons have a smaller wavelength than light enabling the interaction with specimens of near atomic resolution $(\sim 0.02 \AA)^{86}$. The electron microscope is conform by three systems: the electron gun, an image producer and an image recorder. The electron gun is at the top of the column and contains the electron source and the condenser; the image producing system encompass the objective, intermediate and projector lenses; and the recording system is integrated by a camera and a fluorescence screen. The TEM works by accelerating down the electron beam coming from the radiation source at a voltage of 80$300 \mathrm{kV}$, and focusing it on the sample with help of the electromagnetic lenses. The transmitted electrons from the sample are recorded and projected onto the screen ${ }^{84,87}$ (Figure 3.11.). Of note, the electron source is sheltered under a vacuum chamber to minimize the collision with gas atoms. 


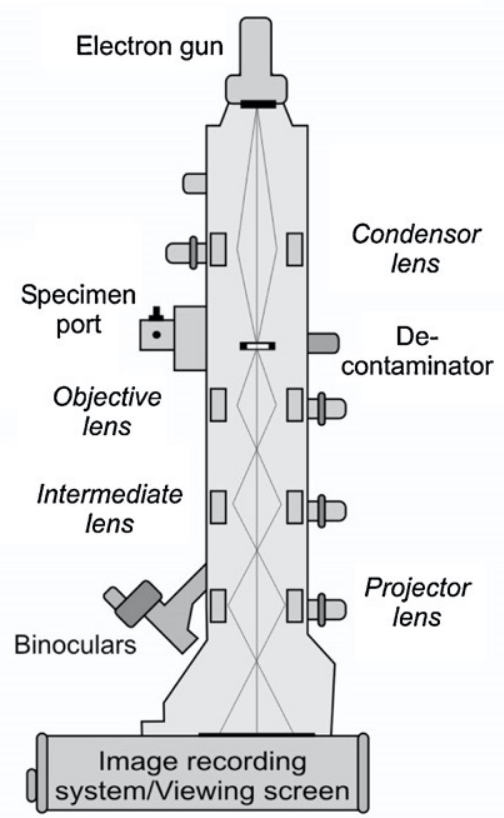

Figure 3.11. Schematic drawing of a transmission electron microscope. The electron light beam is accelerated down the microscope and the electromagnetic lenses focus the beam onto the specimen. The recording system shoots and produce the image on screen. Image retrieved and modified from Kuntsche et al. $(2011)^{87}$.

The sample preparation is done by vitrifying the specimen, of a typical concentration of 1$2 \mathrm{mg} / \mathrm{ml}^{88}$. The first step is the preparation of the cryo grid. Carbon coated holey grids made of copper are commonly used for cryo-TEM. They must be hydrophilized to ease the spreading of the sample by performing a glow discharge. Later, the grid is placed in the automated vitrification machine, and 2 to $5 \mu$ of the sample are pipetted into the grid. The excess of the sample is blotted with a filter paper, leaving a thin film in the grid. Worth noting that samples should be of nanometer size and not too viscous to avoid the removal of big particles when blotting. The grid containing the sample is immediately plunged into liquid ethane, and after being frozen is removed, blotted with filter paper and stored in liquid nitrogen ${ }^{87}$. The reason of using liquid ethane instead of nitrogen is because of its higher heat capacity and liquid state at higher temperatures ${ }^{89}$. Once the sample has been prepared it can be stored for an indefinite period of time until imaging with the electron microscope. Some criteria that should be taken into account when operating the TEM are the usage of low dose imaging conditions to avoid electron beam damage over the sample; 
and a careful initial overview to localize the interesting parts of the film ${ }^{90,91}$.

In brief, cryo-TEM is a highly valuable technique to characterize the morphology of very small samples with a minimum amount of volume. Since the sample is under hydrated conditions and not in contact with adhering surfaces it is not flattened, and as a result the observed shape is the true shape $\mathrm{e}^{92,93}$. The technique was used in this work to characterize and visualize the morphology of bicellar samples. The data helped to build up on the previous DLS results.

\section{Experimental Protocol:}

In chapter 6 , the morphology of the DOPC/GML bicelles with a q-ratio of 0.25 was described by cryo-TEM. The sample was vitrified using a Vitrobot Mark IV (Thermo Fisher, Waltham, MA). Initially, a $4 \mu 1$ volume at the highest concentration of the bicellar mixture was placed onto a cooper holy carbon grid (Quantioil R2/2, Jena, DE). The cryogrid was blotted with filter paper to enable the formation of a thin film of the sample and full coverage of the grid holes. The blotting time was 2 seconds without force. The film was frozen in liquid ethane $\left(-196{ }^{\circ} \mathrm{C}\right)$ and kept in liquid $\mathrm{N}_{2}$ until examination. The visualization of the sample was done with a microscope Tecnai Arctica (Thermo Fisher, Waltham, MA) at $200 \mathrm{kV}$ using low-dose imaging conditions. Approximately 5 overviews and $\sim 60$ electron micrographs were taken.

\subsubsection{Cell Viability Testing}

Cell viability is defined as the number of living cells in a certain population ${ }^{94}$. The amount of proliferating cells is used as a marker of cell survival or death in response to the interaction with a drug or chemical agent. The effect of a drug can be cytostatic or cytotoxic, inhibiting growth or killing the cell, respectively. As with the minimum inhibitory and bactericidal concentrations, the same compound can be cytostatic and cytotoxic depending on its concentration. The use of cytotoxicity and proliferation tests is a staple step in drug development to move forward and try in-vivo models. The detection methods for cytotoxicity and proliferation are classified in four categories: dye exclusion, 
colorimetric, fluorometric and luminometric assays ${ }^{95}$. The selection of the appropriate method depends on the experimental requirements of the sample, mechanism of detection, degree of sensitivity and of course, equipment availability . The colorimetric assay with WST-8 from the cell counting kit-8 (CCK-8) (Dojindo Molecular Technologies, Rockville, MD) was chosen for this study for several reasons. It is not cell permeable, and thus, has no toxic effect over cells, is very sensitive, nonradioactive and minimum preparation is needed. After assessment, the cells can be reuse for other experiments. The compound, WST-8 [2-(2-methoxy-4-nitrophenyl)-3-(4-nitrophenyl)-5-(2,4-disulfophenyl)-2Htetrazolium, monosodium salt] is reduced by dehydrogenase in cells to produce formazan ${ }^{96}$ (Figure 3.12.). The cofactors, $\operatorname{NAD}(\mathrm{H})$ and $\operatorname{NADP}(\mathrm{H})$, and the electron mediator, 1Methoxy PMS, ease the transportation of two electrons from the viable cells to react with WST-8. The product of the reaction is seen with the naked eye by the color change of the culture medium to orange. The number of live cells is directly proportional to the amount of formazan $\operatorname{stain}^{97,98}$ (Figure 3.13.). The quantitative estimation is finished by measuring the absorbance with a plate reader at $450 \mathrm{~nm}$.

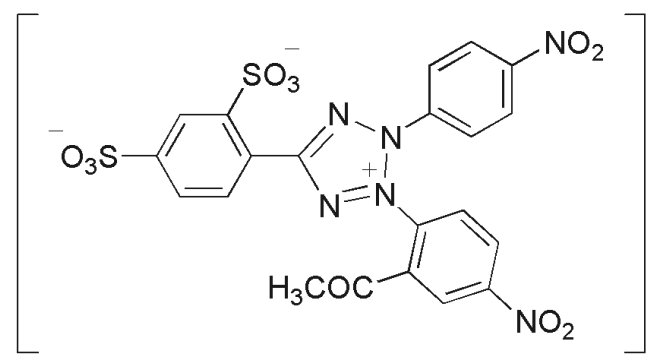

WST-8

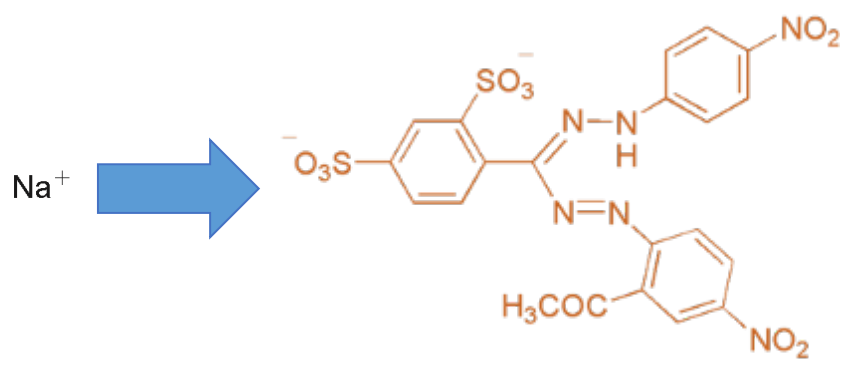

WST-8 formazan

Figure 3.12. Chemical structure of WST-8 and WST-8 formazan. Tetrazolium salt used to determine the number of live and dead skin cells by its reduction with dehydrogenase to produce WST-8 formazan. 


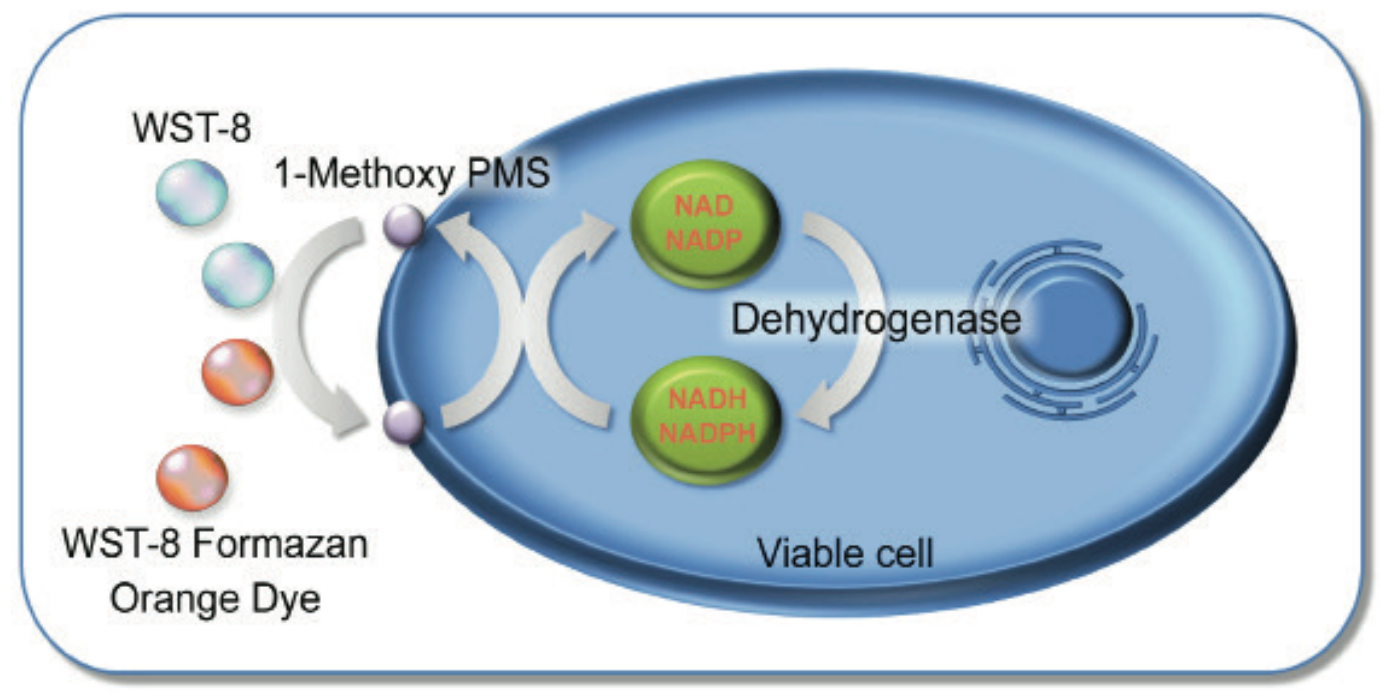

Figure 3.13. Principle of the cell viability detection with the kit CCK-8. WST-8 receives two electrons from the viable cells to generate formazan. The electron mediator, 1-Methoxy PMS, and the cofactors, $\mathrm{NAD}(\mathrm{H})$ and $\mathrm{NADP}(\mathrm{H})$, aid in the catalysis of the reduction reaction. Retrieved from Dojindo's cell counting kit-8 (1963) ${ }^{99}$

The cell viability and cytotoxicity test was performed to study the effect of the bicellar mixture of DOPC and GML (q-ratio of 0.25 ) over the HaCaT cell line. Human immortalized keratinocytes $(\mathrm{HaCaT})$ are a nontumorigenic cell line from epidermal skin cells, adapted for long-term growth ${ }^{100,101}$. It is a reliable model for in-vitro evaluation of skin and new products ${ }^{102}$. The HaCaT cells were cultured according to the recommended specification from the supplier. The medium was Minimum Essential Medium (MEM) supplemented with fetal bovine serum and the antibiotics, penicillin and streptomycin ${ }^{103}$. The Minimum Essential Medium (MEM) was developed for the maintenance and growth of mammalian cells under in-vitro settings. The mixture contains 6 salts, 13 essential amino acids, 8 vitamins and glucose ${ }^{104}$. The fetal bovine serum is the liquid fraction of clotted blood from fetal calves. It contains a large volume of growth factors essential for the maintenance and proliferation of tissue culture ${ }^{105}$. The antibiotics, penicillin and streptomycin, are used in conjunction to prevent microbial contamination ${ }^{106}$. Penicillin is a beta-lactam and kills an extensive range of bacteria by disrupting their membrane ${ }^{107}$. Streptomycin is a broad spectrum aminoglycoside which stops the synthesis of protein in bacteria ${ }^{108}$. The subculture and passage of HaCaT cells was done using trypsin EDTA, which is a proteolytic enzyme that removes calcium and magnesium, favoring the 
detachment of cells from the flask ${ }^{109}$. Lastly, the positive control included in the study was Triton X-100. It is a nonionic surfactant, commonly used as lysing agent for proteins, bacteria and tissue culture ${ }^{110}$.

\section{Experimental Protocol:}

Human immortalized keratinocytes cells (HaCaT) (CLS Cell Lines Service, Eppelheim, Germany) were maintained in Minimum Essential Medium (MEM) supplemented with $10 \%$ fetal bovine serum, $100 \mathrm{U} \mathrm{mL}^{-1}$ penicillin and $100 \mathrm{mg} \mathrm{mL}^{-1}$ streptomycin in a humidified atmosphere with $5 \% \mathrm{CO}_{2}$ at $37^{\circ} \mathrm{C}$. Cells were subculture from the culture flask using $0.25 \%$ Trypsin-EDTA solution. Cell proliferation and viability were quantified using the cell counting kit-8 assay (CCK-8) (Dojindo Molecular Technologies, Rockville, MD). A HaCaT density of 5000 cells per well were seeded in a 96 well plate and allowed to grow overnight. The next days, cell were treated with different concentration of the DOPC/GML (q-ratio 0.25 ) bicelles $-0.5 \mathrm{mM}$ to $2 \mathrm{mM}$ - and further incubated for $72 \mathrm{hrs}$. Cells treated with Triton X-100 were used as positive control. CCK reagent was diluted 10 times with culture media and incubated with cells for $1 \mathrm{~h}$ at $37^{\circ} \mathrm{C}$, the colorimetric culture media absorbance were measured with a plate reader (Infinite 200 PRO microplate reader, Tecan, Zurich, Switzerland).) at $450 \mathrm{~nm}$ wavelength. Experiments were conducted in triplicate and graph results can be found in chapter 6. 


\section{References}

1 Yoon, B. K., Jackman, J. A., Kim, M. C. \& Cho, N.-J. Spectrum of membrane morphological responses to antibacterial fatty acids and related surfactants. Langmuir 31, 10223-10232 (2015).

2 Barbosa-Barros, L. et al. Effect of bicellar systems on skin properties. International journal of pharmaceutics 352, 263-272 (2008).

3 Bower, V. E., Paabo, M. \& Bates, R. A standard for the measurement of the $\mathrm{pH}$ of blood and other physiological media. J. Res. Natl. Bur. Stand. A 65, 267-270 (1961).

4 Zhang, X.-Y. et al. Bacterial Concentration Detection using a PCB-based Contactless Conductivity Sensor. Micromachines 10, 55 (2019).

5 Treangen, T. J. et al. Complete genome sequence of the quality control strain Staphylococcus aureus subsp. aureus ATCC 25923. Genome Announc. 2, e0111001114 (2014).

6 European Pharmacopeia Section 2, 1 (Sterility). (Maisonneuve SA Sainte Ruffine, 2004).

7 Dagnelie, M.-A., Khammari, A., Dréno, B. \& Corvec, S. Assessment of seven protocols to prepare Cutibacterium acnes bacterial lysates to measure its immunogenic potential and review of the literature. Anaerobe 57, 75-81 (2019).

8 Vorum, H., Brodersen, R., Kragh-Hansen, U. \& Pedersen, A. O. Solubility of longchain fatty acids in phosphate buffer at $\mathrm{pH}$ 7.4. Biochimica et Biophysica Acta (BBA)-Lipids and Lipid Metabolism 1126, 135-142 (1992).

9 Chen, C.-H. et al. An innate bactericidal oleic acid effective against skin infection of methicillin-resistant Staphylococcus aureus: a therapy concordant with evolutionary medicine. J. Microbiol. Biotechnol 21, 391-399 (2011).

10 Pornpattananangkul, D. et al. In vivo treatment of Propionibacterium acnes infection with liposomal lauric acids. Advanced healthcare materials 2, 1322-1328 (2013).

11 Nakatsuji, T. et al. Antimicrobial property of lauric acid against Propionibacterium acnes: its therapeutic potential for inflammatory acne vulgaris. Journal of investigative dermatology 129, 2480-2488 (2009). 
12 Schmid, M.-H. \& Korting, H. Therapeutic progress with topical liposome drugs for skin disease. Advanced drug delivery reviews 18, 335-342 (1996).

13 Williams, A. C. \& Barry, B. W. Penetration enhancers. Advanced drug delivery reviews 64, 128-137 (2012).

14 Kabara, J. J., Swieczkowski, D. M., Conley, A. J. \& Truant, J. P. Fatty acids and derivatives as antimicrobial agents. Antimicrobial agents and chemotherapy 2, 2328 (1972).

15 Neuman, M. G. et al. Ethanol signals for apoptosis in cultured skin cells. Alcohol 26, 179-190 (2002).

$16 \mathrm{Wu}, \mathrm{D}$. \& Cederbaum, A. I. Alcohol, oxidative stress, and free radical damage. Alcohol Research \& Health 27, 277 (2003).

17 Abels, C. et al. A 10\% glycolic acid containing oil-in-water emulsion improves mild acne: a randomized double-blind placebo-controlled trial. Journal of cosmetic dermatology 10, 202-209 (2011).

18 Takenaka, Y., Hayashi, N., Takeda, M., Ashikaga, S. \& Kawashima, M. Glycolic acid chemical peeling improves inflammatory acne eruptions through its inhibitory and bactericidal effects on Propionibacterium acnes. The Journal of dermatology 39, 350-354 (2012).

19 Mäler, L. \& Gräslund, A. in Macromolecular Drug Delivery 129-139 (Springer, 2009).

20 De Angelis, A. A. \& Opella, S. J. Bicelle samples for solid-state NMR of membrane proteins. Nature protocols 2, 2332 (2007).

21 Triba, M. N., Warschawski, D. E. \& Devaux, P. F. Reinvestigation by phosphorus NMR of lipid distribution in bicelles. Biophysical journal 88, 1887-1901 (2005).

22 Kolahdouzan, K. et al. Optimizing the formation of supported lipid bilayers from bicellar mixtures. Langmuir 33, 5052-5064 (2017).

23 Lood, R. \& Collin, M. Characterization and genome sequencing of two Propionibacterium acnes phages displaying pseudolysogeny. BMC genomics 12, 198 (2011).

24 Chen, C.-Y., Yan, X. \& Jackson, C. R. Antimicrobial Resistance and Food Safety: Methods and Techniques. (Elsevier, 2015). 
25 Moroi, Y. Micelles: theoretical and applied aspects. (Springer Science \& Business Media, 1992).

26 Thormar, H. Lipids and essential oils as antimicrobial agents. (John Wiley \& Sons, 2010).

27 Fisicaro, E. et al. Thermodynamics of micelle formation in water, hydrophobic processes and surfactant self-assemblies. Physical Chemistry Chemical Physics 10, 3903-3914 (2008).

28 Desbois, A. P. \& Smith, V. J. Antibacterial free fatty acids: activities, mechanisms of action and biotechnological potential. Applied microbiology and biotechnology 85, 1629-1642 (2010).

29 Sanderson, M. J., Smith, I., Parker, I. \& Bootman, M. D. Fluorescence microscopy. Cold Spring Harbor protocols 2014, pdb.top071795-pdb.top071795, doi:10.1101/pdb.top071795 (2014).

30 Lichtman, J. W. \& Conchello, J.-A. Fluorescence microscopy. Nature methods 2 , 910-919 (2005).

31 Ananthapadmanabhan, K., Goddard, E., Turro, N. \& Kuo, P. Fluorescence probes for critical micelle concentration. Langmuir 1, 352-355 (1985).

32 Hermanson, G. T. Bioconjugate techniques. Vol. Chapter 10 395-463 (Academic press, 2013).

33 Malmberg, C. \& Maryott, A. with a maximum deviation of 0.01 unit in dielectric constant. The experimental method and sources of error are considered in some detail. Journal of research of the National Bureau of Standards 56, 1 (1956).

34 Jennings, P. A., Mullen, C. A. \& Roy, M. Titration and p H Measurement. e LS (2001).

35 Sutton, S. C. (Wiley Online Library, 2013).

36 Dixon, M. C. Quartz crystal microbalance with dissipation monitoring: enabling real-time characterization of biological materials and their interactions. Journal of biomolecular techniques: JBT 19, 151 (2008).

37 Lack, F., Willard, G. \& Fair, I. Some improvements in quartz crystal circuit elements. The Bell System Technical Journal 13, 453-463 (1934).

38 Czanderna, A. \& Lu, C. in Methods and Phenomena Vol. 7 1-18 (Elsevier, 1984). 
39 Nomura, T. \& Hattori, O. Determination of micromolar concentrations of cyanide in solution with a piezoelectric detector. Analytica chimica acta 115, 323-326 (1980).

40 Rodahl, M. \& Kasemo, B. A simple setup to simultaneously measure the resonant frequency and the absolute dissipation factor of a quartz crystal microbalance. Review of Scientific Instruments 67, 3238-3241 (1996).

41 Rodahl, M., Höök, F., Krozer, A., Brzezinski, P. \& Kasemo, B. Quartz crystal microbalance setup for frequency and Q-factor measurements in gaseous and liquid environments. Review of Scientific Instruments 66, 3924-3930 (1995).

42 Cho, N.-J., Frank, C. W., Kasemo, B. \& Höök, F. Quartz crystal microbalance with dissipation monitoring of supported lipid bilayers on various substrates. nature protocols 5, 1096-1106 (2010).

43 Davidson, M. W. \& Abramowitz, M. Optical microscopy. Encyclopedia of imaging science and technology (2002).

44 Spring, K., R. and Davidson, M.,W. Introduction to Fluorescence Microscopy, $<$ https://www.microscopyu.com/techniques/fluorescence/introduction-to-

fluorescence-microscopy $>$ (

45 Sanderson, M. J., Smith, I., Parker, I. \& Bootman, M. D. Fluorescence microscopy. Cold Spring Harbor Protocols 2014, pdb. top071795 (2014).

46 Database, J. S. E. (JoVE, Cambridge, MA, 2020).

47 Massari, S., Colonna, R. \& Folena, E. Interaction of the fluorescent probe N(lissamine rhodamine B sulfonyl) dipalmitoylphosphatidylethanolamine with phosphatidylcholine bilayers. Biochimica et Biophysica Acta (BBA)Biomembranes 940, 149-157 (1988).

48 Wiegand, I., Hilpert, K. \& Hancock, R. E. Agar and broth dilution methods to determine the minimal inhibitory concentration (MIC) of antimicrobial substances. Nature protocols 3, 163-175 (2008).

49 CaLSI, C. Performance standards for antimicrobial susceptibility testing. NCCLS document 31M100 - S21. Wayne, National Committee for Clinical Laboratory Standards (2011). 
50 Microbiology, E. C. f. A. S. T. o. t. E. S. o. C. \& Diseases, I. Determination of minimum inhibitory concentrations (MICs) of antibacterial agents by broth dilution. Clinical Microbiology and Infection 9, ix-xv (2003).

51 Hecht, D. W. et al. Methods for Antimicrobial Susceptibiluty Testing of Anaerobic Bacteria: Approved Standard. (Clinical and Laboratory Standards Institute Wayne, PA, 2007).

52 Yang, D. et al. The antimicrobial activity of liposomal lauric acids against Propionibacterium acnes. Biomaterials 30, 6035-6040 (2009).

53 Jensen, M. G. Characterization of health-associated Propionibacterium acnes strains.

54 Standards, N. C. f. C. L. \& Barry, A. L. Methods for determining bactericidal activity of antimicrobial agents: approved guideline. Vol. 19 (National Committee for Clinical Laboratory Standards Wayne, PA, 1999).

55 Pankey, G. A. \& Sabath, L. D. Clinical Relevance of Bacteriostatic versus Bactericidal Mechanisms of Action in the Treatment of Gram-Positive Bacterial Infections. Clinical Infectious Diseases 38, 864-870, doi:10.1086/381972 (2004).

56 Balouiri, M., Sadiki, M. \& Ibnsouda, S. K. Methods for in vitro evaluating antimicrobial activity: A review. Journal of pharmaceutical analysis 6, 71-79 (2016).

57 Berney, M., Hammes, F., Bosshard, F., Weilenmann, H.-U. \& Egli, T. Assessment and interpretation of bacterial viability by using the LIVE/DEAD BacLight Kit in combination with flow cytometry. Appl. Environ. Microbiol. 73, 3283-3290 (2007).

58 Brunius, G. Technical aspects of the use of 3', 6'-diacetyl fluorescein for vital fluorescent staining of bacteria. Current Microbiology 4, 321-323 (1980).

59 Davies, C. M. A comparison of fluorochromes for direct viable counts by image analysis. Letters in applied microbiology 13, 58-61 (1991).

60 Kaprelyants, A. \& Kell, D. Rapid assessment of bacterial viability and vitality by rhodamine 123 and flow cytometry. Journal of Applied Bacteriology 72, 410-422 (1992).

61 Probes, M. LIVE/DEAD BacLight bacterial viability kits. Fluorescence Spectroscopy Protocols. Staining Bacteria with either Kit L7007 or L 7012 (2004). 
62 Menéndez, G. O., Leskow, F. C. \& Spagnuolo, C. C. in Fluorescence Imaging for Surgeons 3-18 (Springer, 2015).

63 Sheppard, C. J. \& Shotton, D. M. Confocal laser scanning microscopy. (1997).

64 Paddock, S. W. Confocal microscopy: methods and protocols. Vol. 122 (Springer Science \& Business Media, 1999).

65 Mempin, R. et al. Release of extracellular ATP by bacteria during growth. BMC Microbiol 13, 301-301, doi:10.1186/1471-2180-13-301 (2013).

66 Bonora, M. et al. ATP synthesis and storage. Purinergic Signal 8, 343-357, doi:10.1007/s11302-012-9305-8 (2012).

67 Atosuo, J., Lehtinen, J., Vojtek, L. \& Lilius, E. M. Escherichia coli K-12 (pEGFPluxABCDEamp): a tool for analysis of bacterial killing by antibacterial agents and human complement activities on a real-time basis. Luminescence $\mathbf{2 8}$, 771-779 (2013).

68 O'neill, A. J., Miller, K., Oliva, B. \& Chopra, I. Comparison of assays for detection of agents causing membrane damage in Staphylococcus aureus. Journal of antimicrobial chemotherapy 54, 1127-1129 (2004).

69 Vojtek, L., Dobes, P., Büyükgüzel, E., Atosuo, J. \& Hyrsl, P. Bioluminescent assay for evaluating antimicrobial activity in insect haemolymph. European Journal of Entomology 111, 335 (2014).

70 Gandelman, O., Allue, I., Bowers, K. \& Cobbold, P. Cytoplasmic factors that affect the intensity and stability of bioluminescence from firefly luciferase in living mammalian cells. Journal of bioluminescence and chemiluminescence 9, 363-371 (1994).

71 Finger, S., Wiegand, C., Buschmann, H.-J. \& Hipler, U.-C. Antibacterial properties of cyclodextrin-antiseptics-complexes determined by microplate laser nephelometry and ATP bioluminescence assay. International Journal of Pharmaceutics 452, 188-193, doi:https://doi.org/10.1016/j.ijpharm.2013.04.080 (2013).

72 Marques, S. M. \& Esteves da Silva, J. C. Firefly bioluminescence: a mechanistic approach of luciferase catalyzed reactions. IUBMB life 61, 6-17 (2009). 
73 Glow Luminescence, $<\underline{\mathrm{https}}$ //www.biotek.com/products/detection-luminescence-

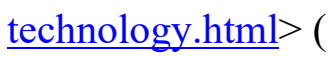

74 Brown, W. Dynamic light scattering: the method and some applications. Vol. 313 (Clarendon Press Oxford, 1993).

75 Stetefeld, J., McKenna, S. A. \& Patel, T. R. Dynamic light scattering: a practical guide and applications in biomedical sciences. Biophys Rev 8, 409-427, doi:10.1007/s12551-016-0218-6 (2016).

76 Technical Committee ISO/TC 24, P. c. i. s. S. S., Particle characterization. Particle size analysis: dynamic light scattering (DLS). (ISO, 2017).

77 Pusey, P. Photon correlation and light beating spectroscopy. Plenum Press, New York (1974).

78 Malvern, I. Dynamic light scattering common terms defined. Worcestershire, UK (2011).

79 Dispersity, $<$ https://iupac.org/100/stories/wikipedia-polymerpartnership/dispersity/> (2018).

U.L., N. POLYDISPERSITY - WHAT DOES IT MEAN FOR DLS AND CHROMATOGRAPHY?, $<\underline{\text { https://Www.materials- }}$ talks.com/blog/2017/10/23/polydispersity-what-does-it-mean-for-dls-andchromatography/> (2017).

81 Fernández, E. et al. Bicelles and bicosomes as free radical scavengers in the skin. RSC advances 4, 53109-53121 (2014).

82 The principles of dynamic light scattering, $<$ https://wiki.anton-paar.com/en/theprinciples-of-dynamic-light-scattering/\#c39795> (

83 (Brookhaven Instruments Analysis of Virus Particles in Media by Dynamic Light Scattering).

84 Milne, J. L. S. et al. Cryo-electron microscopy--a primer for the non-microscopist. FEBS J 280, 28-45, doi:10.1111/febs.12078 (2013).

85 Agard, D., Cheng, Y., Glaeser, R. M. \& Subramaniam, S. in Advances in imaging and electron physics Vol. 185 113-137 (Elsevier, 2014). 
86 Henderson, R. et al. Model for the structure of bacteriorhodopsin based on highresolution electron cryo-microscopy. Journal of molecular biology 213, 899-929 (1990).

87 Kuntsche, J., Horst, J. C. \& Bunjes, H. Cryogenic transmission electron microscopy (cryo-TEM) for studying the morphology of colloidal drug delivery systems. International journal of pharmaceutics 417, 120-137 (2011).

88 Harris, J. R. Negative staining and cryoelectron microscopy. Microscopy Today 5, 18-18 (1997).

89 Dubochet, J. et al. Cryo-electron microscopy of vitrified specimens. Quarterly reviews of biophysics $\mathbf{2 1}, 129-228$ (1988).

90 Chen, J. Z. et al. A dose-rate effect in single-particle electron microscopy. J Struct Biol 161, 92-100, doi:10.1016/j.jsb.2007.09.017 (2008).

91 Orlova, E. V. \& Saibil, H. R. Structural analysis of macromolecular assemblies by electron microscopy. Chemical reviews 111, 7710-7748, doi:10.1021/cr100353t (2011).

92 Thompson, R. F., Walker, M., Siebert, C. A., Muench, S. P. \& Ranson, N. A. An introduction to sample preparation and imaging by cryo-electron microscopy for structural biology. Methods 100, 3-15, doi:10.1016/j.ymeth.2016.02.017 (2016).

93 Cryo-Electron Microscopy, <https://www.microscopemaster.com/cryo-electronmicroscopy.html $>($

94 Adan, A., Kiraz, Y. \& Baran, Y. Cell proliferation and cytotoxicity assays. Current pharmaceutical biotechnology 17, 1213-1221 (2016).

95 Aslantürk, Ö. S. In vitro cytotoxicity and cell viability assays: principles, advantages, and disadvantages. Vol. 2 (InTech, 2018).

96 (Dojindo Molecular Technologies, Inc, 2016).

97 Ishiyama, M., Miyazono, Y., Sasamoto, K., Ohkura, Y.\& Ueno, K. A highly watersoluble disulfonated tetrazolium salt as a chromogenic indicator for NADH as well as cell viability. Talanta 44, 1299-1305 (1997).

98 Tominaga, H. et al. A water-soluble tetrazolium salt useful for colorimetric cell viability assay. Analytical Communications 36, 47-50 (1999).

99 Cell Proliferation \& Cell Cytotoxicity. Dojindo's cell counting kit-8 (1963). 
100 Schürer, N., Köhne, A., Schliep, V., Barlag, K. \& Goerz, G. Lipid composition and synthesis of HaCaT cells, an immortalized human keratinocyte line, in comparison with normal human adult keratinocytes. Experimental dermatology 2, 179-185 (1993).

101 Micallef, L. et al. Effects of extracellular calcium on the growth-differentiation switch in immortalized keratinocyte HaCaT cells compared with normal human keratinocytes. Experimental dermatology 18, 143-151 (2009).

102 Colombo, I. et al. HaCaT Cells as a Reliable In Vitro Differentiation Model to Dissect the Inflammatory/Repair Response of Human Keratinocytes. Mediators Inflamm 2017, 7435621-7435621, doi:10.1155/2017/7435621 (2017).

103 in CLS Cell Lines Service GmbH (CLS Cell Lines Service GmbH ).

104 Eagle, H. Amino acid metabolism in mammalian cell cultures. Science 130, 432437 (1959).

105 Johnson, M. Fetal bovine serum. Mater Methods 2, 117 (2012).

106 Perlman, D. in Methods in enzymology Vol. 58 110-116 (Elsevier, 1979).

107 Hugo, W. \& Russell, A. The mode of action of penicillin. Journal of Pharmacy and Pharmacology 13, 705-722 (1961).

108 Parker, J. in Encyclopedia of Genetics (eds Sydney Brenner \& Jefferey H. Miller) 1890-1891 (Academic Press, 2001).

109 Fedoroff, S. \& Richardson, A. Protocols for neural cell culture. 342 (Springer Science \& Business Media, 2008).

110 Koley, D. \& Bard, A. J. Triton X-100 concentration effects on membrane permeability of a single HeLa cell by scanning electrochemical microscopy (SECM). Proceedings of the National Academy of Sciences 107, 16783, doi:10.1073/pnas.1011614107 (2010). 


\section{Chapter 4}

\section{Characterizing How Acidic pH Conditions Affect the Membrane-Disruptive Activities of Lauric Acid and Glycerol Monolaurate*}

Medium-chain saturated fatty acids and monoglycerides are present on the human skin surface as part of the innate immune system. They exhibit antibacterial activity by destabilizing bacterial cell membranes and supported lipid bilayers (SLBS) provide a useful platform to characterize their behaviour. Herein, it was investigated how lauric acid (LA) fatty acid and glycerol monolaurate (GML) monoglyceride interact with SLB platforms and cause membrane morphological changes under acidic $\mathrm{pH}$ conditions mimicking the human skin environment. The findings are discussed in the context of how solution $p H$ affects the ionization states and micellar aggregation properties of LA and GML as well as its effect on the bending stiffness of lipid bilayers. The antibacterial activities of LA and GML against Cutibacterium acnes -bacteria causing acne vulgaris-were also tested. The results agreed well with the biophysical findings and indicated that GML exhibits superior inhibitory activity against $C$. acnes. Collectively, the findings demonstrate that solution $\mathrm{pH}$ plays an important role in modulating the interaction of fatty acids and monoglycerides with lipid membranes, and hence influences the scope and potency of membrane-disruptive activities and related biological activities.

*This chapter is published substantially as Valle-González, E. R.; Jackman, J. A.; Yoon, B. K.; Park, S.; Sut, T. N.; Cho, N.-J., Characterizing How Acidic pH Conditions Affect the Membrane-Disruptive Activities of Lauric Acid and Glycerol Monolaurate. Langmuir 2018, 34 (45), 13745-13753. DOI: 10.1021/acs.langmuir.8b02536. Reproduced with permission, Copyright (2018) American Chemical Society. 


\subsection{Introduction}

The self-assembly of lipid molecules plays an important role in driving membrane $\operatorname{organization}^{1,2}$ and facilitating biomedical applications such as drug delivery ${ }^{3}$ and antimicrobial medicine. ${ }^{4}$ Among different types of lipid molecules, antimicrobial lipids are single-chain lipid amphiphiles - most commonly free fatty acids and their monoglyceride derivatives - that interact with phospholipid membranes, including bacterial cell membranes. ${ }^{5,6}$ It has been demonstrated that antimicrobial lipids can kill a broad spectrum of bacterial, viral, and fungal pathogens, and have found utility for anti-infective applications. ${ }^{7,8}$ Antimicrobial lipids are also an integral part of innate immunity on human skin surfaces, where their main function is to protect against pathogenic species and regulate microbial populations. ${ }^{9-11}$ Among the range of antimicrobial lipids, it has been discovered that medium-chain saturated fatty acids and monoglycerides with 10- and 12carbon long hydrocarbon chains have particularly high antibacterial activities. ${ }^{12}$ Lauric acid (LA; C12:0) is regarded as the most inhibitory saturated fatty acid against Gram-positive bacteria. ${ }^{13}$ Its monoglyceride derivative, glycerol monolaurate (GML), also has potent antibacterial effects; GML demonstrates greater potency (lower effective concentration) albeit against a narrower spectrum of susceptible bacteria. ${ }^{13-16}$ Biologically, it has been established that antimicrobial lipids, including LA and GML, inhibit bacterial cell growth by partially solubilizing the membrane (termed "bacteriostatic") or cause membrane lysis, resulting in rapid cell death (termed "bactericidal"). ${ }^{17}$

Conventionally, the effects of antimicrobial lipids on bacterial cell membranes have been assessed post-treatment by electron microscopy (EM) techniques, which enable visualization of membrane morphological changes and intracellular damage. ${ }^{18,19} \mathrm{EM}$ studies have revealed that antimicrobial lipids, including LA and GML, can cross the peptidoglycan cell wall of Gram-positive bacteria, leading to membrane solubilisation and release of cytoplasmic contents. ${ }^{18-20}$ However, real-time monitoring of membrane interactions is not possible with EM, and similar challenges also exist for atomic force microscopy approaches. ${ }^{21}$ To overcome these challenges, supported lipid bilayers (SLBs) provide a versatile, two-dimensional model membrane platform that is compatible with a 
variety of surface-sensitive measurement techniques. The SLB platform provides a single lipid bilayer that mimics the key structural component of Gram-positive bacterial cell membranes and hence changes in model membrane morphology can provide insight into potential antibacterial activities. A common experimental approach has been to utilize quartz crystal microbalance-dissipation (QCM-D) experiments to monitor in real-time the binding interactions and subsequent membrane morphological responses via changes in the mass and viscoelastic properties of the SLB platform, while time-lapsed fluorescence microscopy is employed to directly observe the type of membrane morphological change. ${ }^{22}$

Based on this approach, Yoon et al. utilized SLB platforms to distinguish the membrane morphological changes caused by fatty acids and monoglycerides. ${ }^{14}$ It was shown that anionic LA induced the formation of elongated tubule structures, whereas nonionic GML caused membrane budding. The distinct membrane morphological responses were attributed to differences in the membrane translocation properties of anionic versus nonionic compounds, ${ }^{23}$ and corresponding effects on membrane strain. ${ }^{24}$ Importantly, the membrane-destabilizing activities of LA and GML were dependent on their respective critical micelle concentration (CMC) values. GML was more potent than LA on account of a lower CMC value, which could be explained by the tendency of nonionic compounds to aggregate more favorably than otherwise similar, anionic compounds. ${ }^{14}$ It was also identified that the CMC values of LA and GML are correlated with their respective minimum inhibitory concentration (MIC) values against Staphylococcus aureus, offering a link between membrane-disruptive and biological activities. Furthermore, it was shown that another fatty acid and monoglyceride pair, capric acid (C10:0) and monocaprin, exhibits similar trends in the type and concentration-dependence of membrane morphological changes. ${ }^{25}$ Collectively, these findings established that the micellar aggregation properties of fatty acids and monoglycerides are intimately connected with how they interact with phospholipid membranes and corresponding biological activities.

To date, all SLB studies involving fatty acids and monoglycerides have been conducted in near-neutral $\mathrm{pH}$ conditions, and there is an outstanding need to further characterize their membrane-disruptive activities in more diverse, biologically relevant conditions. For 
example, fatty acids and monoglycerides are biologically active on the skin surface, which is an acidic environment and $\mathrm{pH} 4.7$ is the optimal value for healthy skin. ${ }^{26}$ Exogenous addition of these compounds also represents a potential therapeutic strategy for topical skin applications. ${ }^{27-29}$ In particular, LA has been reported to have high antibacterial activity against Gram-positive Cutibacterium acnes, which is the cause of acne vulgaris. ${ }^{27,30-33}$ Considering that the acid dissociation constant of fatty acids lies in the acidic regime, it is important to characterize the interaction of LA with lipid membranes in acidic $\mathrm{pH}$ conditions, and to understand how its $\mathrm{pH}$-dependent ionization state might affect membrane-disruptive activities and related biological activities. Indeed, LA possesses an ionizable carboxylic acid functional group and can exist in neutral or anionic states depending on solution $\mathrm{pH}$ (Figure 4.1.). GML is another promising antimicrobial lipid that warrants further investigation due to its high potency, although its possible effect on $C$. acnes infection has not yet been reported. In contrast to LA, GML does not possess an ionizable functional group and understanding how solution $\mathrm{pH}$ affects its membranedisruptive activity would also provide insight into how $\mathrm{pH}$-dependent lipid bilayer properties affect membrane morphological changes.

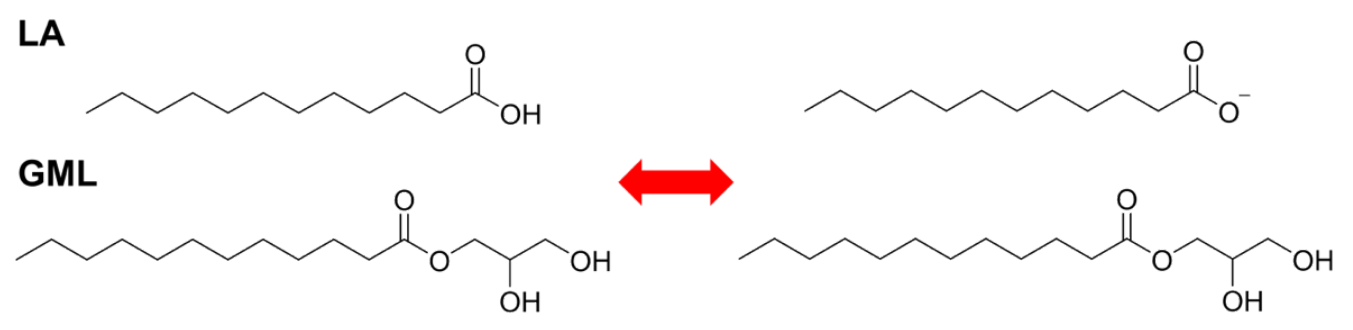

Low pH

High pH

Figure 4.1. Molecular structures of LA and GML under acidic (4.5) and near-neutral (7.4) pH conditions. LA has an ionizable carboxylic acid group that undergoes protonation/deprotonation depending on the $\mathrm{pH}$ condition. By contrast, GML is nonionic.

To address these outstanding questions, the objective of this chapter was to investigate how LA and GML interact with SLB platforms under acidic $\mathrm{pH}$ conditions. Multiple biophysical measurement techniques were applied to characterize membrane-disruptive 
activities, and comparative measurements were made in near-neutral $\mathrm{pH}$ conditions. The results enabled to determine how acidic $\mathrm{pH}$ conditions influence membrane-disruptive activities, including characterizing the potency and types of membrane morphological changes that are caused by LA and GML along with their corresponding micellar aggregation properties. The antibacterial properties of LA and GML against C. acnes were also investigated. Taken together, this integrated experimental approach allowed to generate mechanistic insights into how solution $\mathrm{pH}$ influences the biophysical and biological activities of antimicrobial lipids.

\subsection{Materials and Methods}

\subsubsection{Materials}

Phospholipids for SLB fabrication were obtained as-supplied in chloroform from Avanti Polar Lipids, Inc. (Alabaster, AL). Lauric acid and glycerol monolaurate were acquired from Sigma-Aldrich (St. Louis, MO) and Abcam (Cambridge, UK), respectively. Tryptic soy broth (TSB), BD GasPak EZ Incubation Container, and GasPak EZ Anaerobe System sachets with indicator were purchased from Becton Dickinson (Franklin Lakes, NJ). Defibrinated sheep blood was obtained from Thermo Fisher Scientific (Waltham, MA). Blood agar plates and 5\% sheep blood in tryptic soy agar base were acquired from Hardy Diagnostics (Santa Maria, CA). Phosphate-buffered saline (PBS) was purchased from Gibco (Carlsbad, CA). All solutions were prepared with Milli-Q-treated deionized water ( $>18 \mathrm{M} \Omega \cdot \mathrm{cm}$ resistivity) (Millipore, Billerica, MA).

\subsubsection{Preparation of Antimicrobial Lipid Solutions}

Stock solutions of LA and GML dissolved in ethanol were prepared by weighing the appropriate amount of lyophilized compound and dissolving in ethanol to a concentration of 400 or $200 \mathrm{mM}$, respectively. Before experiment, aliquots were diluted 100-fold with PBS buffer solution to the highest test concentration of $4 \mathrm{mM}$ and $2 \mathrm{mM}$ for LA and GML, respectively. To promote solubilisation, the samples were heated to $70{ }^{\circ} \mathrm{C}$ for $30 \mathrm{~min}$, and 
then cooled down before measurements were conducted at room temperature.

\subsubsection{Fluorescence Spectroscopy}

The CMC values of LA and GML in the appropriate $\mathrm{pH}$ conditions were determined by fluorescence spectroscopy measurements, which were conducted using a Cary Eclipse fluorescence spectrophotometer (Varian, Inc., Australia). Specifically, 1-pyrene carboxaldehyde was employed as a fluorescent probe that is sensitive to the presence of micellar aggregates. The test samples were prepared by adding a small aliquot of the fluorescent probe $(5 \mathrm{mM})$ in methanol to a glass tube, and then the methanol was evaporated to form a dry thin film of the probe compound on the glass walls. Afterwards, the designated concentration of LA or GML in the appropriate solution was added to the vial, and the sample was vortexed followed by heating to $70{ }^{\circ} \mathrm{C}$. Finally, the sample was cooled down before measurements were conducted at room temperature. The final concentration of the probe was $0.1 \mu \mathrm{M}$. In the measurements, the test samples were excited at $365.5 \mathrm{~nm}$ and the emission spectrum was recorded from 400 to $600 \mathrm{~nm}$. The highestintensity wavelength in the emission spectrum was recorded for each measurement, and the mean and standard deviation are reported from six technical replicates.

\subsection{4. pH Titration method}

A stock solution of lauric acid in water $(5 \mathrm{mM}, 20 \mathrm{~mL})$ was titrated with a $0.5,2.5$ and 5 $\mathrm{mM} \mathrm{NaOH}$ solution. The $\mathrm{NaOH}$ was added stepwise until the titration curve reached plateau. The change in $\mathrm{pH}$ of each solution was recorded using an Accumet AB15 pH meter (Thermo Fisher Scientific; Waltham, MA).

\subsubsection{Quartz Crystal Microbalance-Dissipation}

QCM-D experiments were performed in order to characterize SLB formation and to detect membrane morphological responses that occurred when test compounds were added to SLB platforms. The measurements were conducted with a four-channel Q-Sense E4 
instrument (Biolin Scientific, Stockholm, Sweden), with simultaneous time-lapsed recording of shifts in the resonance frequency $(\Delta f)$ and energy dissipation $(\Delta D)$ of an oscillating, piezoelectric quartz crystal. ${ }^{34}$ The sensor chips had a fundamental frequency of $5 \mathrm{MHz}$, and were coated with a sputter-coated, 50-nm-thick layer of silicon dioxide (model no. QSX 303, Biolin Scientific). Before experiment, the chips were sequentially rinsed with water and ethanol, dried with a stream of nitrogen gas, and treated with oxygen plasma for 1 min using an Expanded Plasma Cleaner (model no. PDC-002, Harrick Plasma, Ithaca, NY). After cleaning, the chips were immediately loaded into the measurement chambers and buffer solution was injected to establish a measurement baseline. All samples were injected at a flow rate of $50 \mu \mathrm{L} / \mathrm{min}$, as controlled by a peristaltic pump (Reglo Digital, Ismatec, Glattbrugg, Switzerland). The temperature in the measurement chamber was maintained at $25.0 \pm 0.5^{\circ} \mathrm{C}$. The measurement data were recorded at the third $(n=3)$, fifth $(n=5)$, and seventh $(n=7)$ odd overtones using the QSoft software program (Biolin Scientific), and the data was normalized according to the overtone number. Data processing was performed in the QTools (Biolin Scientific) and OriginPro 8.5 (OriginLab, Northampton, MA) software programs. All presented data was collected at the fifth overtone.

\subsubsection{Fluorescence Microscopy}

Epifluorescence microscopy experiments were conducted in order to directly observe morphological changes in SLBs on glass surfaces upon treatment with LA or GML. The experiments were conducted using an Eclipse TI-U inverted optical microscope (Nikon, Japan) with a $60 \times$ magnification $(\mathrm{NA}=1.49)$ oil-immersion objective lens (Nikon), and images were collected with an iXon 512 pixel $\times 512$ pixel EMCCD camera (Andor Technology, Northern Ireland). The pixel size was $0.267 \times 0.267 \mu \mathrm{m}^{2}$. A fiber-coupled mercury lamp (Intensilight C-HGFIE, Nikon) was used to illuminate fluorescently labeled phospholipids (a small fraction of all phospholipids in the SLB) with a TRITC filter. For the experiments, the SLB was fabricated following the vesicle fusion method with DOPC, and $0.5 \mathrm{~mol} \%$ of the fluorophore, 1,2-dipalmitoyl-sn-glycero-3- phosphoethanolamine-N(lissamine rhodamine B sulfonyl) lipid. The vesicles were prepared with Tris buffer (150 
$\mathrm{mM} \mathrm{NaCl}, \mathrm{pH} \mathrm{7.5)}$ and extruded through $50 \mathrm{~nm}$ diameter polycarbonate membranes (Whatman Schleicher \& Schuell, Dassel, Germany). The resulting vesicles had an average diameter of $60 \mathrm{~nm}$ and the solution a concentration of $0.2 \mathrm{mg} / \mathrm{ml}$. SLBs were formed on a glass slide that was enclosed within a microfluidic flow-through chamber (sticky slide VI 0.4, Ibidi, Germany). After SLB formation, the measurement chamber was rinsed with PBS buffer solution, and then the test compound was added under continuous flow conditions at a flow rate of $40 \mu \mathrm{L} / \mathrm{min}$. Time-lapse micrographs were recorded every $5 \mathrm{~s}$ for a total duration of $30 \mathrm{~min}$, and the initial time, $t=0 \mathrm{~s}$, was defined by when the test compound solution reached the measurement chamber. The fluorescence intensity profile of each micrograph was normalized using a custom-written script for the Python(x,y) 2.7.5 software program.

\subsubsection{Bacterial Cell Culture}

A quality control strain of C. acnes (ATCC 11827; American Type Culture Collection, Manassas, VA) was cultured in Tryptic soy broth with 5\% defibrinated sheep blood for 48 hrs under anaerobic conditions using a Gas-Pak $\left(80 \% \mathrm{~N}_{2}, 13 \% \mathrm{CO}_{2}, 7 \% \mathrm{H}_{2}\right)$ at $37{ }^{\circ} \mathrm{C}$. The bacterial suspension was then re-inoculated in fresh Tryptic soy broth with 5\% defibrinated sheep blood and cultured under the same anaerobic conditions for an additional $24 \mathrm{hrs}$. The bacterial cells were next harvested by centrifugation at $1485 \times g$ for $10 \mathrm{~min}$, washed thrice with PBS, and re-suspended in the same buffer. The $\mathrm{OD}_{600}$ value was measured and the appropriate dilution was made to reach a value of approximately 0.35 (mid-exponential growth phase). ${ }^{35}$ This value corresponds to a density of $2 \times 10^{8} \mathrm{CFU} / \mathrm{mL}$ (CFU: colonyforming units), as confirmed by CFU enumeration in control experiments. A 100-fold PBS

dilution was performed to dilute the cells to a density of $2 \times 10^{6} \mathrm{CFU} / \mathrm{mL}$ for testing purposes. 


\subsubsection{Minimum Inhibitory Concentration (MIC) Assay}

The MIC values of antimicrobial lipids against $C$. acnes were determined by using the agar plate method. The test compounds were initially diluted with PBS across the concentration range from $4 \mathrm{mM}$ to $8 \mu \mathrm{M}$ in a two-fold dilution series. An equivalent volume of $C$. acnes at a concentration of $2 \times 10^{6} \mathrm{CFU} / \mathrm{mL}$ was added to each well, yielding a final cell density of $1 \times 10^{6} \mathrm{CFU} / \mathrm{mL}$. The plates were cultured for $1 \mathrm{hr}$ under anaerobic conditions at $37^{\circ} \mathrm{C}$, and then spotted on Tryptic soy agar plates supplemented with 5\% defibrinated sheep blood. The $1 \mathrm{hr}$ incubation time facilitated sufficient contact time prior to cell culture because membrane-disruptive antimicrobial lipids typically kill susceptible bacteria within 30 min or less. ${ }^{36,37}$ Afterwards, the agar plate culture method facilitated C. acnes growth in a suitable anaerobic environment. ${ }^{38}$ The streaked plates were incubated under anaerobic conditions at $37{ }^{\circ} \mathrm{C}$ for 4 days, after which the presence of bacterial colonies in each test group was evaluated. The MIC values were determined by identifying the lowest concentration of test compound that completely inhibited the visible growth of bacterial colonies.

\subsection{Results and Discussion}

\subsubsection{Experimental Strategy}

The experimental strategy involved a combination of biophysical and biological measurement techniques, as illustrated in Figure 4.2. Fluorescence spectroscopy experiments were conducted using an environment-sensitive fluorescent probe to determine the CMC values of LA and GML in appropriate $\mathrm{pH}$ conditions. To investigate membrane-disruptive activities, QCM-D experiments were performed to track the effects of concentration-dependent LA and GML treatment on the mass and viscoelastic properties of SLB platforms. As follow-up, time-lapsed fluorescence microscopy experiments were conducted to observe the membrane morphological changes in SLB platforms, which arose from treatment with selected concentrations of LA and GML. The minimum concentrations 
of LA and GML that were necessary to inhibit growth of $C$. acnes at the tested $\mathrm{pH}$ conditions were also determined.
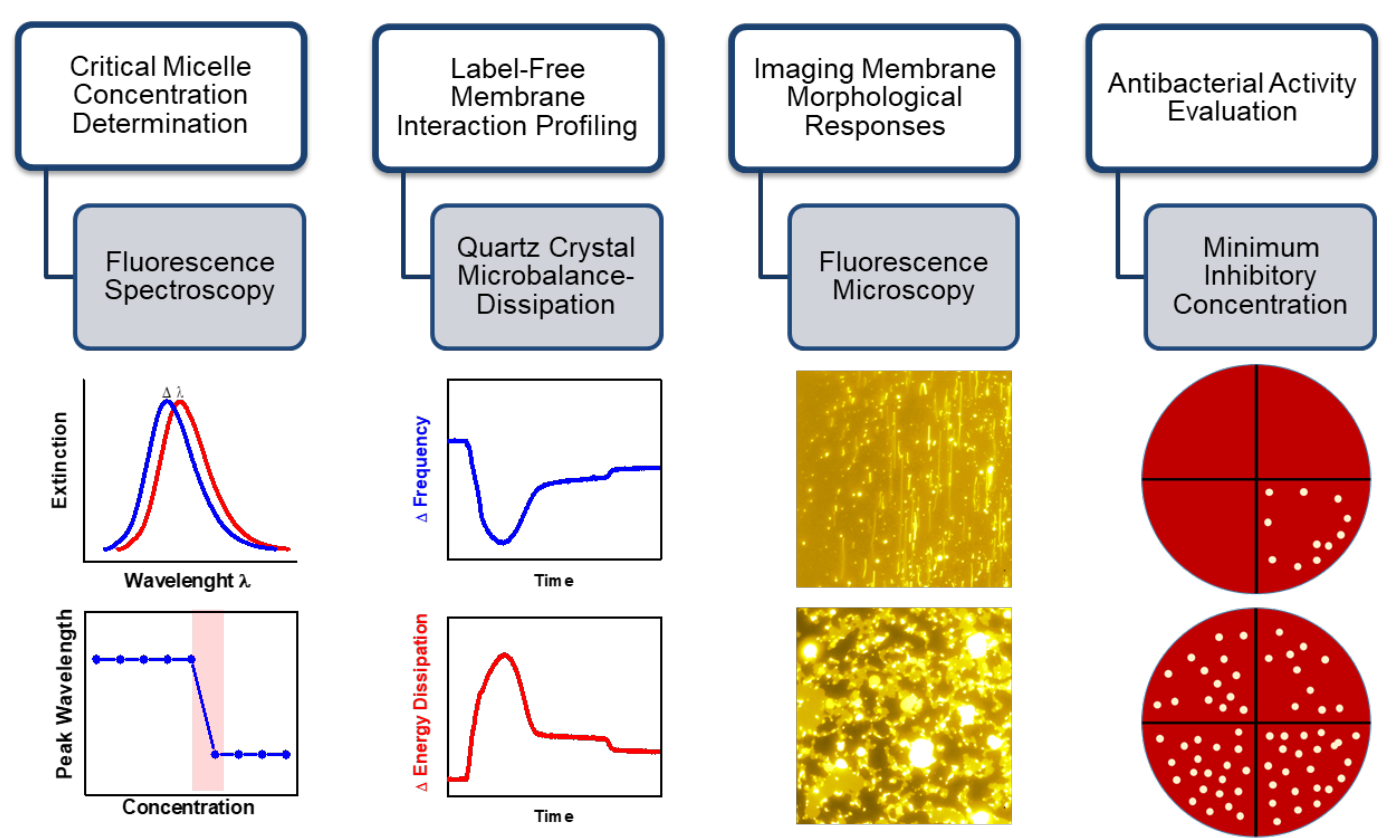

Figure 4.2. Overview of experimental strategy integrating biophysical and biological measurement approaches.

\subsubsection{Effect of Solution pH on Critical Micelle Concentration}

To determine how solution $\mathrm{pH}$ affects the CMC values of LA and GML, fluorescence spectroscopy experiments were conducted based on detecting the partitioning of a fluorescent molecule, 1-pyrene carboxaldehyde, into micellar aggregates (Figure 4.3.). In aqueous environments, 1-pyrene carboxaldehyde has a peak emission wavelength around $473 \mathrm{~nm}$, and the peak wavelength decreases in the presence of micellar aggregates as the dielectric constant of the surrounding environment becomes lower. ${ }^{39}$ Hence, the CMC value of a test compound in particular aqueous conditions can be readily assessed by incubating different concentrations of the compound with the fluorescent probe and defining the $\mathrm{CMC}$ as the lowest concentration at which the peak wavelength begins to decrease relative to the baseline value. The $\mathrm{CMC}$ experiments were conducted in $\mathrm{pH} 7.4$ 
and 4.5 conditions that encompass typical values for physiological (blood) and skin $\mathrm{pH}$ levels, respectively.

Following this approach, the CMC value of LA was measured in $\mathrm{pH} 7.4$ conditions and determined a CMC value of $900 \mu \mathrm{M}$, which agrees well with previous literature values that were obtained in similar ionic strength and $\mathrm{pH}$ conditions ${ }^{40}$ (Figure 4.3A). By contrast, in $\mathrm{pH} 4.5$ conditions, the CMC value of LA decreased to $100 \mu \mathrm{M}$, demonstrating that micellar aggregation of LA is more thermodynamically favorable under acidic $\mathrm{pH}$ conditions (Figure 4.3B). This trend is consistent with the ionization properties of LA as it is known that the $\mathrm{pK}_{\mathrm{a}}$ value of LA is around $\sim 5$ (refs. ${ }^{41,42}$ ). The $\mathrm{pK}_{\mathrm{a}}$ value of LA is further verified in the next section.

In addition, the CMC value of GML was measured in $\mathrm{pH} 7.4$ and 4.5 conditions, and was found to be $60 \mu \mathrm{M}$ in both cases (Figures 4.3C,D). This finding is consistent with GML's molecular properties as a nonionic compound that is formed by the esterification of lauric acid and glycerol, and it is chemically unreactive across the tested $\mathrm{pH}$ range. Taken together, the experimental results demonstrate that the micellar aggregation properties of LA and GML exhibit differing sensitivities to solution $\mathrm{pH}$, and these differences can be rationalized by taking into account the molecular properties of the two compounds. It is particularly noteworthy that the CMC value of LA shifts down to $100 \mu \mathrm{M}$ at $\mathrm{pH} 4.5$, suggesting that it may have more potent membrane-disruptive activities under acidic conditions. 

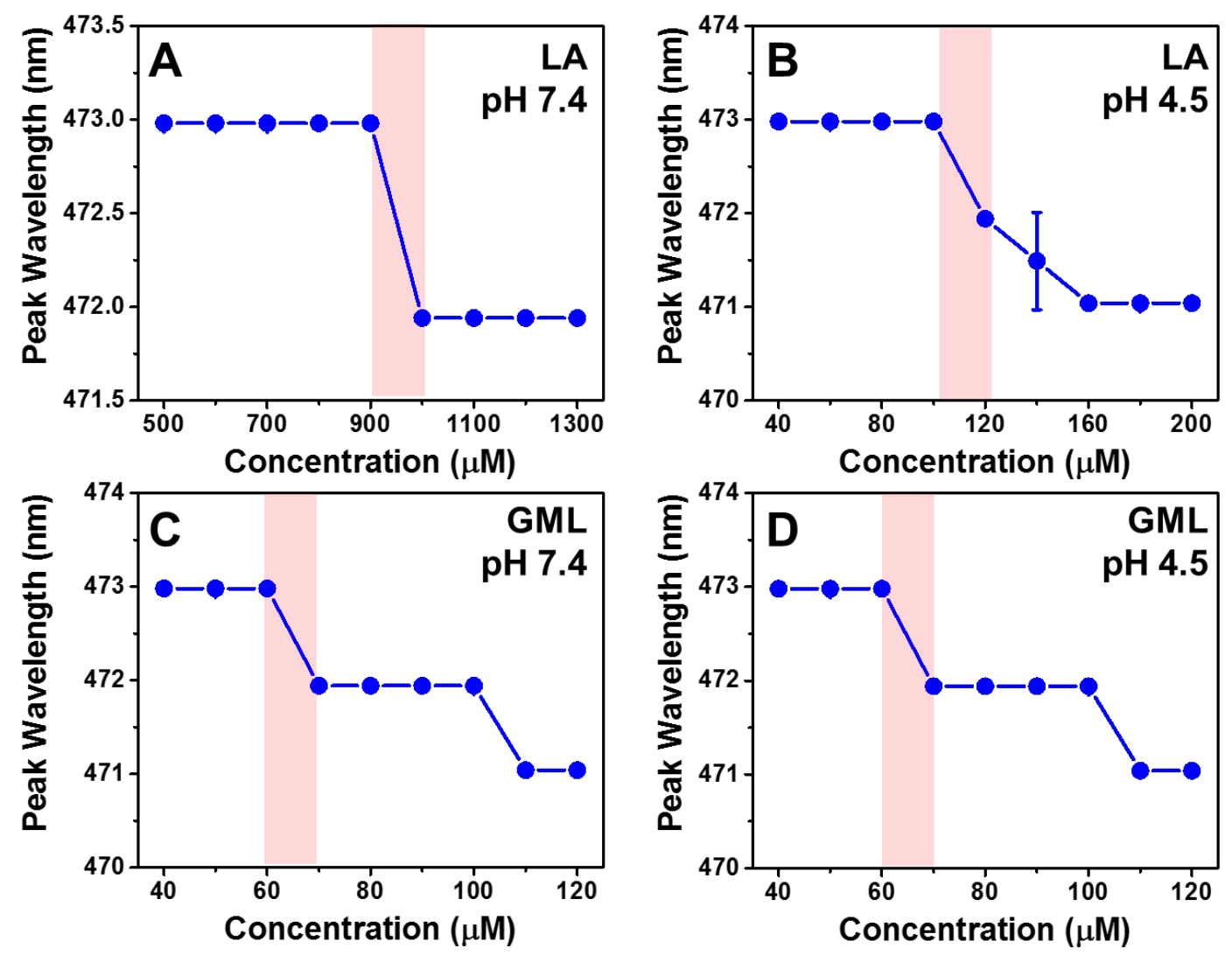

Figure 4.3. Determination of critical micelle concentration for LA and GML in different pH environments. Peak wavelength is shown as a function of compound concentration in PBS solution for (A) LA at pH 7.4, (B) LA at pH 4.5, (C) GML at pH 7.4, and (D) GML at pH 4.5. Each point in the graph represents the average value of six scans $(n=6)$. Standard deviations (expressed as the error bars) are presented where applicable. The CMC value is defined as the highest test concentration before the first break point occurs.

\subsubsection{Determination of the $\mathrm{pK}_{\mathrm{a}}$ value of Lauric acid with the $\mathrm{pH}$ Titration method}

To calculate the $\mathrm{pK}_{\mathrm{a}}$ value of LA and corroborate the $\mathrm{pH}$-dependent CMC results, $5 \mathrm{mM}$ LA was volumetrically titrated with different $\mathrm{NaOH}$ solutions. The solution $\mathrm{pH}$ was raised from 5.4 until reaching a plateau at $\sim 8.6$. The obtained readings were graphed as a function of titrant volume in order to calculate the $\mathrm{pK}_{\mathrm{a}}$ values with the Henderson-Hasselbach equation (Figure 4.4.). Depending on the $\mathrm{NaOH}$ concentration added during the titration, the determined $\mathrm{pK}_{\mathrm{a}}$ value slightly varied and the mean value was $\sim 5.8$. The $\mathrm{pK}_{\mathrm{a}}$ represents the condition when half of the LA molecules are protonated and the other half are 
deprotonated. The Henderson-Hasselbach equation also provides an estimated percentage of LA disassociation, with $95 \%$ protonated $\mathrm{LA}$ at $\mathrm{pH} 4.5$ and $98 \%$ deprotonated $\mathrm{LA}$ at $\mathrm{pH}$ $7.4^{43}$
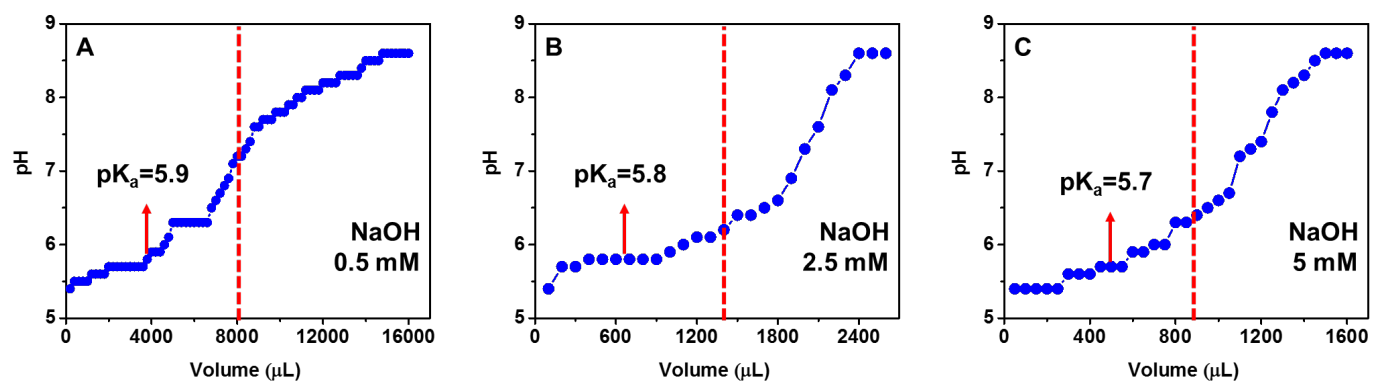

Figure 4.4. Determination of the $\mathbf{p K}_{\mathrm{a}}$ value of lauric acid in water. Titration curves were established, measuring the solution $\mathrm{pH}$ as a function of the volume of $0.5,2.5$ and $5 \mathrm{mM} \mathrm{NaOH}$ added to the solution. The $\mathrm{pH}$ of the inflection point was determined to be $\sim 7$ (corresponding to complete ionization of LA), and the $\mathrm{pH}$ value corresponding to half of the $\mathrm{NaOH}$ volume that was necessary to reach the inflection point was defined as the $\mathrm{pK}_{\mathrm{a}}$ value. Following this approach, the average $\mathrm{pK}_{\mathrm{a}}$ value obtained from the three curves was defined to be 5.8.

Equation 4.1. Henderson-Hasselbach equation, percentage of disassociation.

$$
\begin{gathered}
p H=p K_{a}+\log _{10}\left(\frac{\left[A^{-}\right]}{[A H]}\right) \\
\left.\left(\frac{\left[A^{-}\right]}{[A H]}\right)=10^{(p K a-p H)}\right) \\
\% \text { Disassociation }=100 \times\left(1-10^{(p K a-p H)}\right) \\
p K_{a}=5.8
\end{gathered}
$$

pH 7.4 = 98\% Deprotonated

pH $4.5=95 \% \quad$ Protonated

Taken together, the findings support that the CMC values recorded for LA at the two tested $\mathrm{pH}$ conditions are consistent with the extent of molecular protonation/deprotonation. 


\subsubsection{Effect of Compound Treatment on Supported Lipid Bilayers}

QCM-D experiments were conducted in order to measure the concentration-dependent activities of LA and GML acting against SLB platforms in $\mathrm{pH} 4.5$ and 7.4 conditions. In QCM-D measurements, changes in resonance frequency $(\Delta f)$ and energy dissipation $(\Delta D)$ of an oscillating quartz crystal sensor chip are tracked as a function of time, and correlate with the acoustic mass (biomolecular mass and hydrodynamically-coupled solvent mass) and viscoelastic properties of an adsorbed thin film on the sensor surface, respectively. A negative $\Delta f$ shift corresponds to an increase in acoustic mass while a positive $\Delta D$ shift relates to an increase in film viscoelasticity. SLBs composed of 1,2-dioleoyl-sn-glycero-3phosphocholine (DOPC) were fabricated at the appropriate $\mathrm{pH}$ condition on silicon dioxide-coated sensor surfaces by the solvent-assisted lipid bilayer (SALB) method, ${ }^{44,45}$ and provided the measurement platform upon which membrane morphological responses were detected. Specifically, the reported baseline measurement values correspond to intact SLB platforms and then LA or GML in equivalent buffer solution were added to the measurement chamber under continuous flow conditions starting at $t=5 \mathrm{~min}$. The resulting $\Delta f$ and $\Delta D$ signals occurred due to membrane morphological changes and the specific type of morphological response was assigned based on previous works. ${ }^{14,25}$ After the measurement response stabilized, a washing step with equivalent buffer solution was performed. The results obtained for LA and GML are presented below.

\section{Lauric Acid}

LA addition to SLBs in the $\mathrm{pH} 7.4$ condition followed established trends showing concentration-dependent tubule formation at $1 \mathrm{mM}$ and higher LA concentrations (Figure 4.5.). Specifically, two-step interaction kinetics were observed whereby the $\Delta f$ shift decreased to $-42 \mathrm{~Hz}$ at the inflection point before increasing. The corresponding $\Delta D$ shifts increased to around $5 \times 10^{-6}$. By contrast, at $500 \mu \mathrm{M} \mathrm{LA}$, the measurement responses were negligible, indicating no interaction in this low concentration regime. These data are consistent with LA being active only at and above its CMC value, which was $900 \mu \mathrm{M}$. 

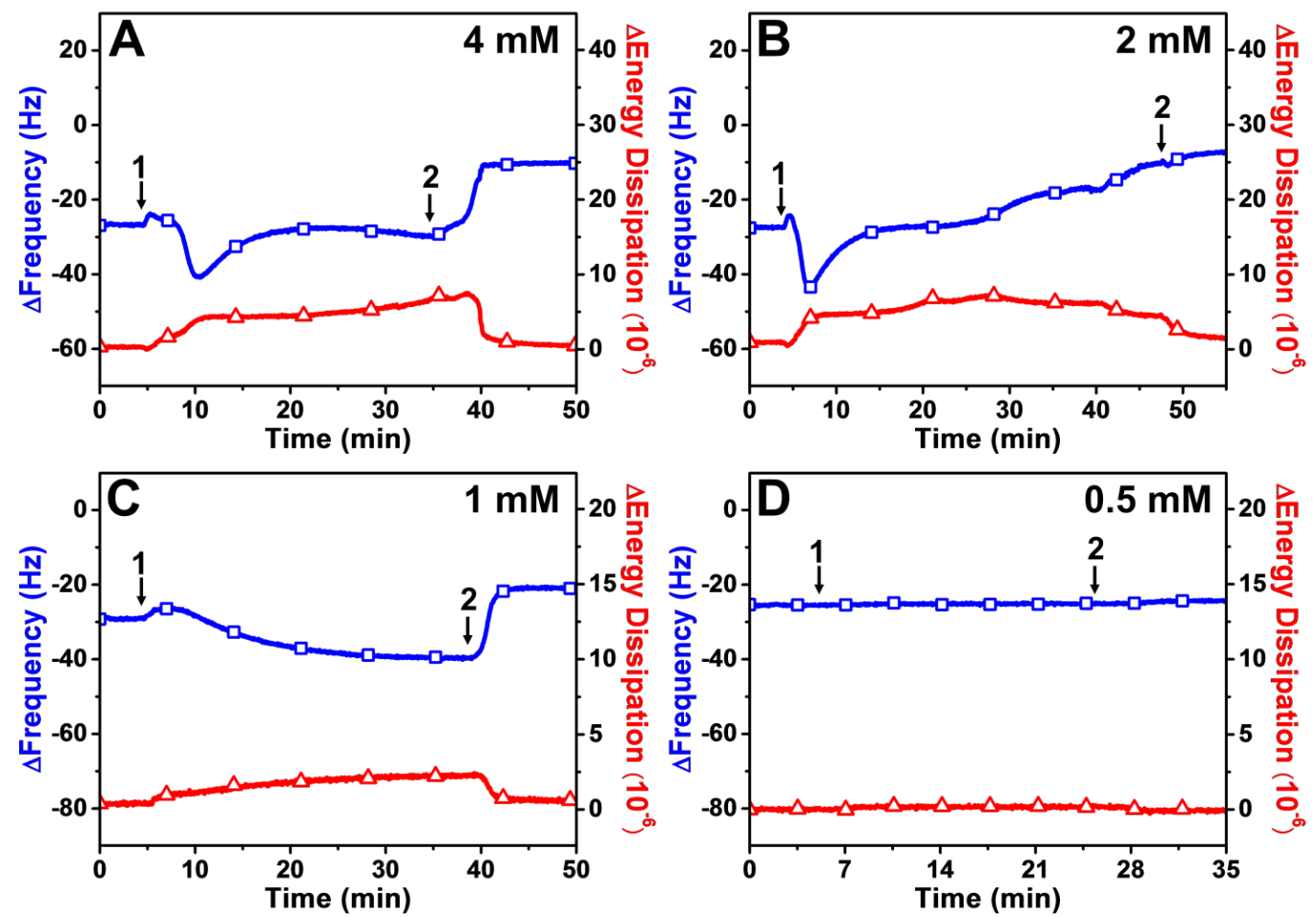

Figure 4.5. QCM-D investigation of lauric acid treatment on supported lipid bilayers at pH

7.4. $\Delta f$ (blue line with squares) and $\Delta D$ (red line with triangles) shifts are presented as a function of time for (A) $4 \mathrm{mM}$, (B) $2 \mathrm{mM}$, (C) $1 \mathrm{mM}$, and (D) $500 \mu \mathrm{M}$ LA. The baseline values at $t=0 \mathrm{~min}$ correspond to an SLB platform on the sensor surface. LA was added at $t=5 \min (\operatorname{arrow} 1)$, and a buffer washing step was performed (arrow 2) after the measurement signals stabilized. 

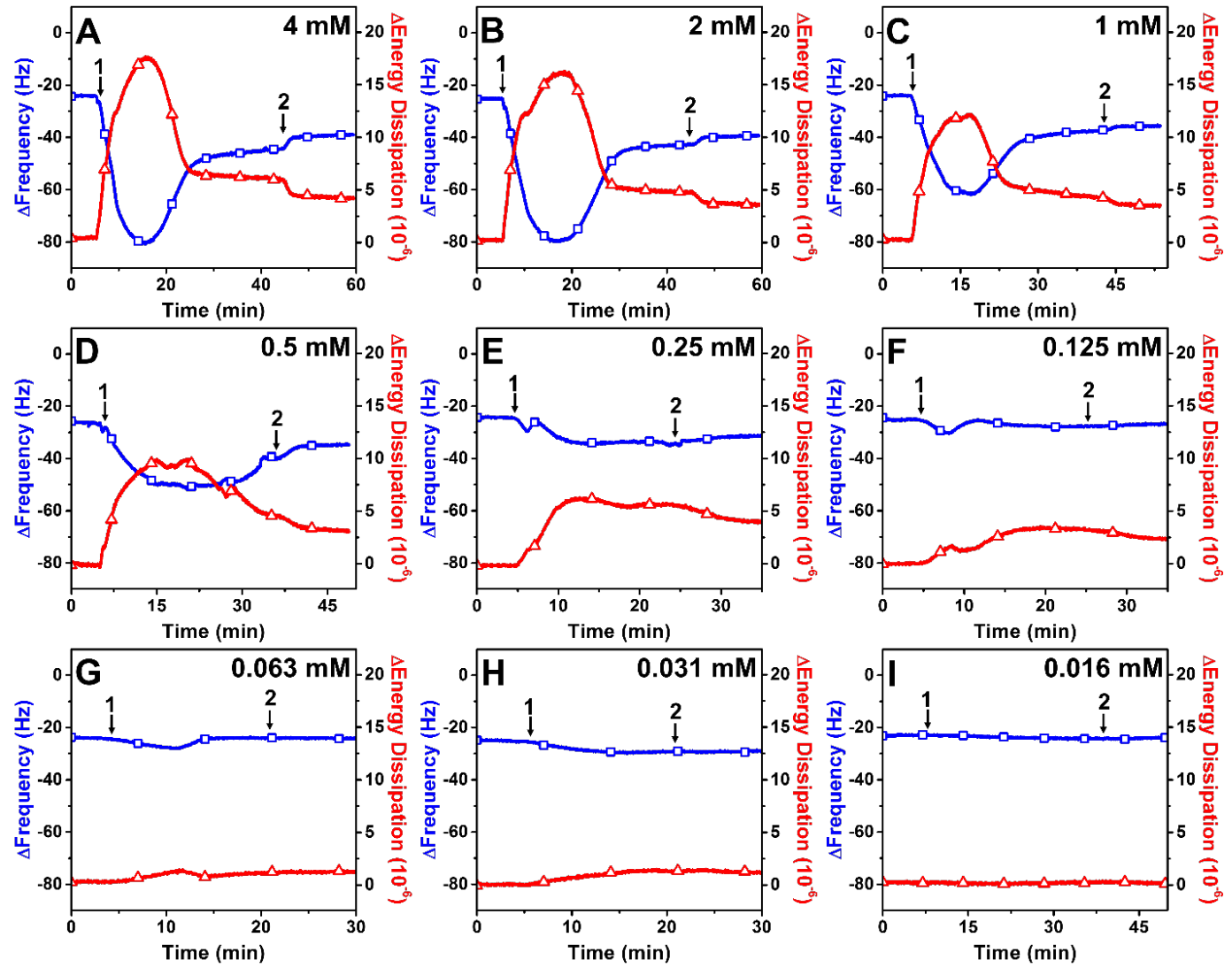

Figure 4.6. QCM-D investigation of lauric acid treatment on supported lipid bilayers at pH

4.5. $\Delta f$ (blue line with squares) and $\Delta D$ (red line with triangles) shifts are presented as a function of time for (A) $4 \mathrm{mM}$, (B) $2 \mathrm{mM}$, (C) $1 \mathrm{mM}$, (D) $500 \mu \mathrm{M}$, (E) $250 \mu \mathrm{M}$, (F) $125 \mu \mathrm{M}$, (G) $63 \mu \mathrm{M}$, (H) $31 \mu \mathrm{M}$, and (I) $16 \mu \mathrm{M} \mathrm{LA}$. The baseline values at $t=0$ min correspond to an SLB platform on the sensor surface. LA was added at $t=5 \mathrm{~min}$ (arrow 1), and a buffer washing step was performed (arrow 2) after the measurement signals stabilized.

In marked contrast, LA addition to SLBs in the $\mathrm{pH} 4.5$ condition induced appreciably larger changes in the $\Delta f$ and $\Delta D$ signals that reached around $-85 \mathrm{~Hz}$ and $17 \times 10^{-6}$, respectively, for $4 \mathrm{mM}$ LA (Figure 4.6A). A subsequent buffer washing step had minor effect on the measurement responses. Similar results were obtained for $2 \mathrm{mM} \mathrm{LA}$ and concentrationdependent responses with matching kinetic profiles were observed down to $500 \mu \mathrm{M}$ LA (Figures 4.6B-D). The magnitude of the measurement responses, particularly the large $\Delta D$ shifts, supports that the resulting membrane morphological responses arise from buddinglike behaviour rather than tubule formation. In the range of 250 to $125 \mu \mathrm{M} \mathrm{LA}$, the $\Delta f$ shifts are appreciably smaller while significant $\Delta D$ shifts were observed around 2 to $5 \times 10^{-6}$ 
(Figures 4.6E, F). These responses suggest a reordering of membrane components, as commonly found for membranes in different phase states. ${ }^{46}$ At lower LA concentrations, the measurement responses were nearly negligible (Figures 4.6G-I). Together, these data support that LA induces distinct membrane morphological responses in the $\mathrm{pH} 4.5$ condition, which resemble membrane budding. This pattern is also consistent with the fact

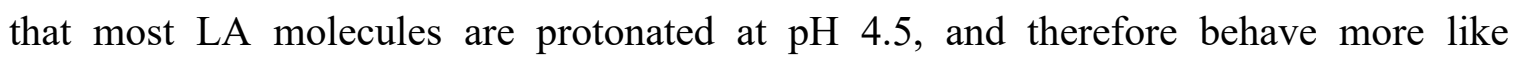
monoglycerides with greater rates of membrane translocation, hence altering the membrane strain profile. Another important finding is that LA was active down to $125 \mu \mathrm{M}$ in the $\mathrm{pH}$ 4.5 condition, and this is also consistent with its corresponding CMC value of $100 \mu \mathrm{M}$. Hence, the data support that LA is more active under acidic conditions, as determined by the lower minimum concentration at which it induces membrane morphological responses.

\section{Glycerol Monolaurate}

GML addition to SLBs in the $\mathrm{pH} 7.4$ condition revealed a combination of membrane budding and membrane-lytic behavior, as evidenced by particularly disruptive activity at 1 $\mathrm{mM}$ and higher GML concentrations and more prominent membrane budding in the range of 500 to $125 \mu \mathrm{M}$ GML (Figure 4.7.). At the higher GML concentrations, two-step interaction kinetics were observed whereby the $\Delta f$ shift decreased to around $-120 \mathrm{~Hz}$ at the inflection point before increasing. ${ }^{47}$ The corresponding $\Delta D$ shifts increased to around $30 \mathrm{x}$ $10^{-6}$. By contrast, at lower GML concentrations, the $\Delta f$ shift reached around $-80 \mathrm{~Hz}$ and the corresponding $\Delta D$ shifts were around $15-25 \times 10^{-6}$, while remaining largely stable thereafter. GML concentration-dependent membrane budding responses were also observed at 63 and $31 \mu \mathrm{M}$ GML, while no activity was observed at $16 \mu \mathrm{M}$ and lower GML concentrations. Taken together, the observed trends support that membrane budding is the predominant membrane morphological response observed upon GML treatment, and the range of active GML concentrations is consistent with past observations that monoglycerides are particularly active at the $\mathrm{CMC}$ and higher while a more moderate degree of activity occurs at slightly lower concentrations as well. 
In the $\mathrm{pH} 4.5$ condition, GML addition also caused membrane budding although the extent of the QCM-D measurement responses was significantly larger. At $2 \mathrm{mM} \mathrm{GML}$, the $\Delta f$ shift decreased to around $-340 \mathrm{~Hz}$ and the corresponding $\Delta D$ shift was around $55 \times 10^{-6}$, with both responses reaching nearly stable values (Figure 4.8A). Upon buffer washing, the $\Delta f$ shift returned to nearly baseline values while the $\Delta D$ shift stabilized around $13 \times 10^{-6}$. Similar measurement responses were observed in a GML concentration-dependent fashion down to $31 \mu \mathrm{M}$ GML (Figures 4.8B-G). The magnitudes of the $\Delta f$ and $\Delta D$ responses in this concentration regime were comparable to those obtained in the $\mathrm{pH} 7.4$ condition. Furthermore, $16 \mu \mathrm{M}$ and lower GML concentrations were inactive against SLBs in the $\mathrm{pH}$ 4.5 condition, demonstrating that GML concentration-dependent activity against SLBs followed identical trends across the two tested $\mathrm{pH}$ conditions (Figures $\mathbf{4 . 8 H}, \mathbf{I}$ ). The only major distinction was that, at higher GML concentrations, greater lytic activity was observed against SLBs in the $\mathrm{pH} 7.4$ condition whereas greater extents of membrane budding occurred in the $\mathrm{pH} 4.5$ condition. This difference likely relates to the fact that the bending stiffness of phosphatidylcholine bilayers can decrease under acidic conditions ${ }^{48,49}$ and hence membrane remodeling could occur to a greater extent in response to GMLinduced membrane strain in this case. 

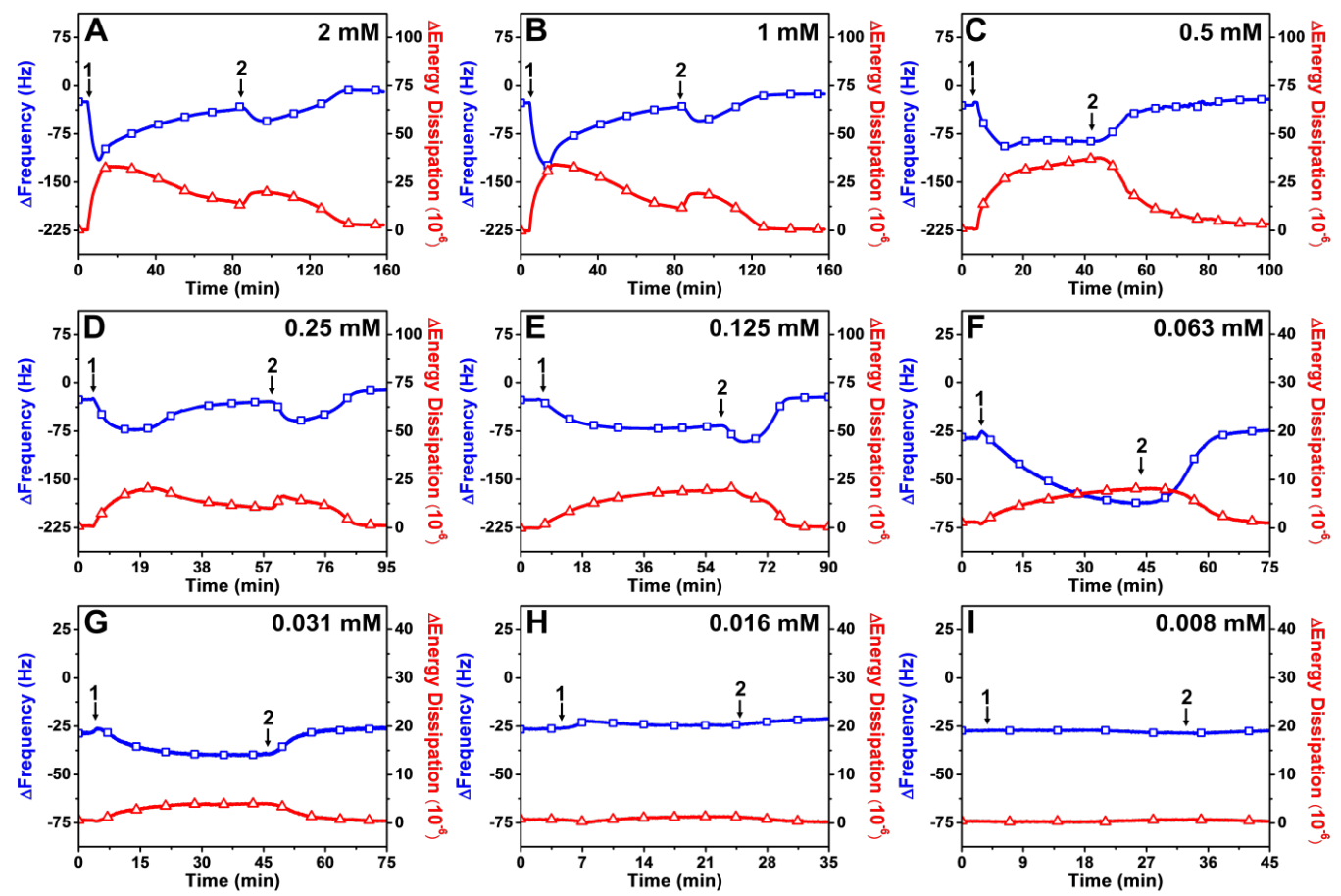

Figure 4.7. QCM-D investigation of glycerol monolaurate treatment on supported lipid

bilayers at pH 7.4. $\Delta f$ (blue line with squares) and $\Delta D$ (red line with triangles) shifts are presented as a function of time for (A) $2 \mathrm{mM}$, (B) $1 \mathrm{mM}$, (C) $500 \mu \mathrm{M}$, (D) $250 \mu \mathrm{M}$, (E) $125 \mu \mathrm{M}$, (F) $63 \mu \mathrm{M}$, (G) $31 \mu \mathrm{M}$, (H) $16 \mu \mathrm{M}$, and (I) $8 \mu \mathrm{M}$ GML. The baseline values at $t=0$ min correspond to an SLB platform on the sensor surface. GML was added at $t=5 \min$ (arrow 1), and a buffer washing step was performed (arrow 2) after the measurement signals stabilized. 

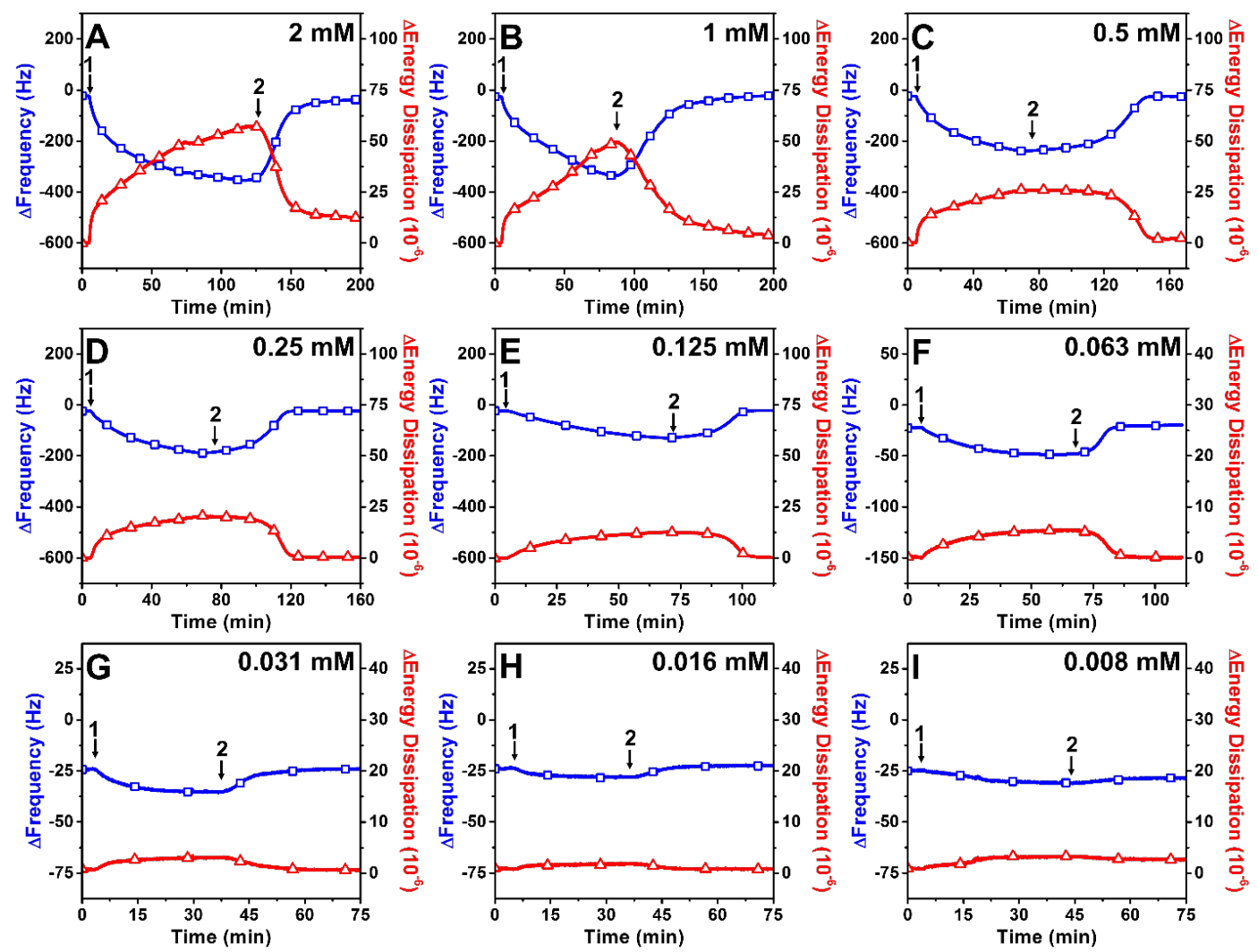

Figure 4.8. QCM-D investigation of glycerol monolaurate treatment on supported lipid bilayers at pH 4.5. $\Delta f$ (blue line with squares) and $\Delta D$ (red line with triangles) shifts are presented as a function of time for (A) $2 \mathrm{mM}$, (B) $1 \mathrm{mM}$, (C) $500 \mu \mathrm{M}$, (D) $250 \mu \mathrm{M}$, (E) $125 \mu \mathrm{M}$, (F) $63 \mu \mathrm{M}$, (G) $31 \mu \mathrm{M}$, (H) $16 \mu \mathrm{M}$, and (I) $8 \mu \mathrm{M}$ GML. The baseline values at $t=0 \min$ correspond to an SLB platform on the sensor surface. GML was added at $t=5 \min$ (arrow 1), and a buffer washing step was performed (arrow 2) after the measurement signals stabilized.

\subsubsection{Observation of Membrane Morphological Responses}

To complement the QCM-D measurements, time-lapsed fluorescence microscopy experiments were conducted to provide direct observation of membrane morphological responses in the SLB platforms. The SLBs were composed of $99.5 \mathrm{~mol} \%$ DOPC and 0.5 mol\% 1,2-dipalmitoyl-sn-glycero-3-phosphoethanolamine- $\mathrm{N}$-(lissamine rhodamine B sulfonyl) phospholipid, and prepared via the vesicle fusion method on a hydrophilic glass surface that was enclosed within a microfluidic chamber to control the introduction of liquid sample. The SLB was formed at the appropriate $\mathrm{pH}$ condition (4.5 or 7.4 depending 
on the experiment) and once a baseline signal was established, either LA or GML in equivalent buffer solution was added under continuous flow conditions. The time point labeled " $t=0$ min" indicates when the solution containing the test compound (either LA or GML) reached the measurement chamber. Based on the fluorescence spectroscopy and QCM-D measurement results, LA was tested at $2 \mathrm{mM}$ and $250 \mu \mathrm{M}$ concentrations. At the former concentration, LA molecules are in the micellar state at both tested $\mathrm{pH}$ conditions, while at the latter concentration, LA molecules are in the micellar state only under the acidic condition. GML was also tested at $500 \mu \mathrm{M}$ at both $\mathrm{pH}$ conditions.

\section{2 mM Lauric Acid}

Figure 4.9A presents time-lapsed snapshots of membrane morphological responses that occur when $2 \mathrm{mM}$ LA was added to an SLB platform in the $\mathrm{pH} 7.4$ condition. Within a few minutes, elongated tubules began to protrude from the SLB platform and became aligned with the flow direction in the microfluidic chamber. After rinsing was performed, the tubules were removed and the underlying SLB platform appeared to be largely intact, as indicated by uniform fluorescence intensity across the bilayer. A few, small bright spots persisted that are likely nucleation sites from which the tubules formed. Overall, the observed results agree well with previous works describing LA treatment of SLB platforms, ${ }^{14}$ and provide control data to compare with membrane morphological responses observed under acidic conditions.

In marked contrast, Figure 4.9B shows that when $2 \mathrm{mM}$ LA was added to an SLB platform in the $\mathrm{pH} 4.5$ condition, small buds formed within $10 \mathrm{~min}$. However, protonated LA molecules induced particular membrane morphological changes that are quite distinct from treatment with monoglycerides. Over time, the number of buds decreased while fluorescence microstructures appeared in the SLB, giving rise to an archipelago effect that was characterized by darker regions with lower fluorophore concentrations and brighter regions with higher fluorophore concentrations. ${ }^{50}$ These features are consistent with phase separation of two coexisting phases with a low line tension, ${ }^{51}$ suggesting that protonated LA molecules intercalate within the SLB. Upon buffer washing, the two coexisting phases 
remained evident and hence LA treatment appeared to induce phase separation in the SLB platform. Hence, under $\mathrm{pH} 4.5$ condition, LA treatment not only induces membrane budding, but also causes phase separation. The observed behaviours are quite distinct from LA treatment at $\mathrm{pH} 7.4$ where tubule formation occurred without apparent phase separation, and are consistent with the predominant ionization state of LA molecules at the low $\mathrm{pH}$ conditions. 


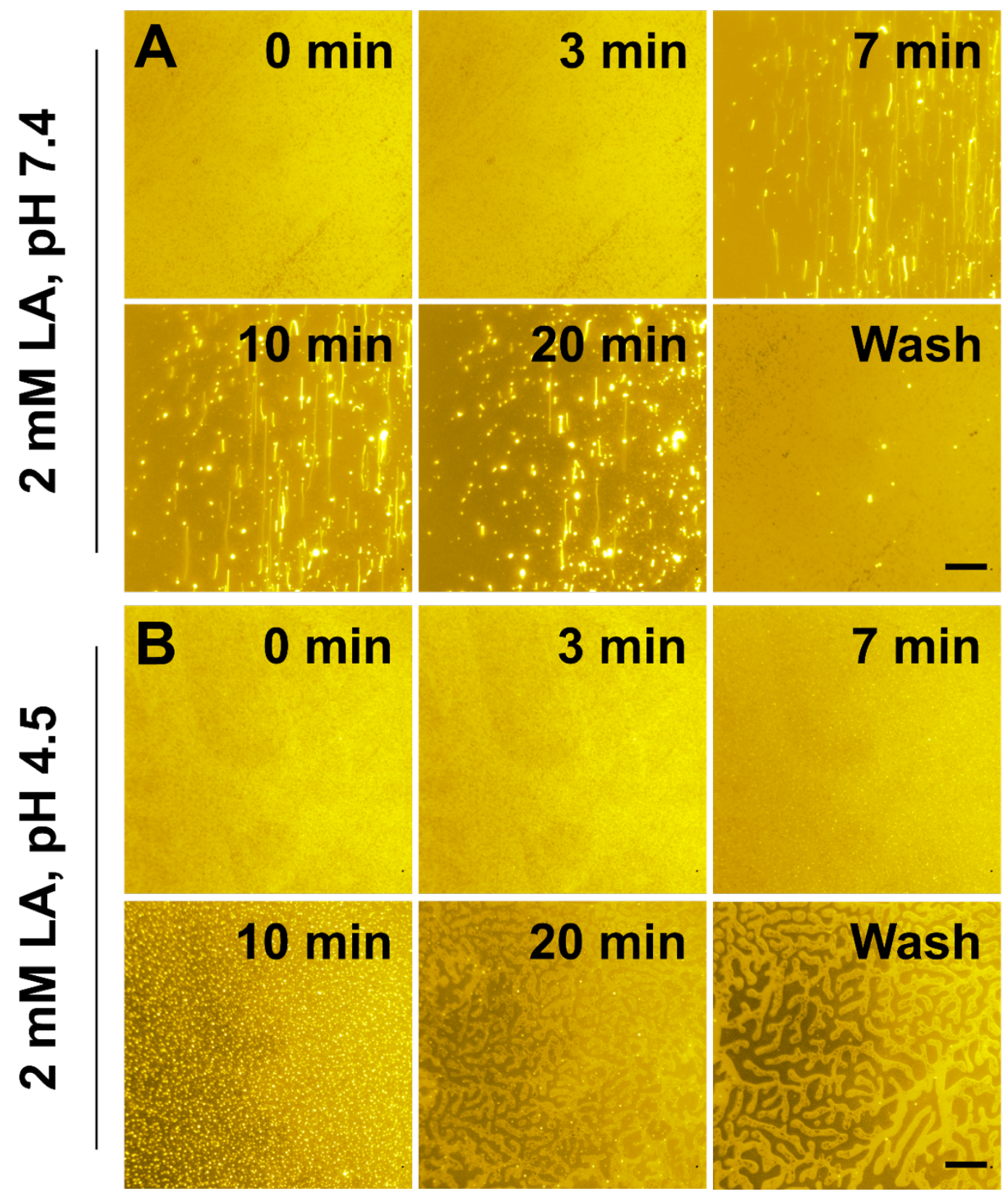

Figure 4.9. Microscopic observation of $2 \mathrm{mM}$ lauric acid-induced membrane morphological responses on supported lipid bilayers. (A) Image snapshots at various time points depict nucleation sites from which tubules grow upon LA treatment of SLB at pH 7.4. (B) Image snapshots at various time points depict nucleation sites from which buds grow and membrane phase separation occurs upon LA treatment of SLB at $\mathrm{pH}$ 4.5. $t=0$ min corresponds to the introduction of $2 \mathrm{mM}$ LA solution into the measurement chamber. The scale bar is $20 \mu \mathrm{m}$. 
$250 \mu$ M Lauric Acid

The effect of $250 \mu \mathrm{M}$ LA treatment was also tested in order to observed membrane morphological responses in a condition where LA is active only under acidic conditions. Indeed, Figure 4.10A shows that $250 \mu \mathrm{M}$ LA treatment has negligible effect on the SLB platform in the $\mathrm{pH} 7.4$ condition, and this lack of activity is consistent with the monomeric state of LA molecules in this condition. By contrast, as presented in Figure 4.10B, $250 \mu \mathrm{M}$ LA treatment of the SLB platform in the $\mathrm{pH} 4.5$ condition induced membrane budding within a few minutes. The buds remained stable and extensive phase separation was not observed during the treatment step. However, upon buffer washing, some evidence of phase separation was observed although striping was not prominent. Together, these results support that LA is more potent against SLBs under acidic conditions, and the observed trends agrees well with the measured CMC values. Hence, LA in acidic conditions induces membrane budding and is active at lower bulk concentrations, whereas LA in neutral conditions causes tubule formation and is only active at appreciably higher bulk concentrations. 


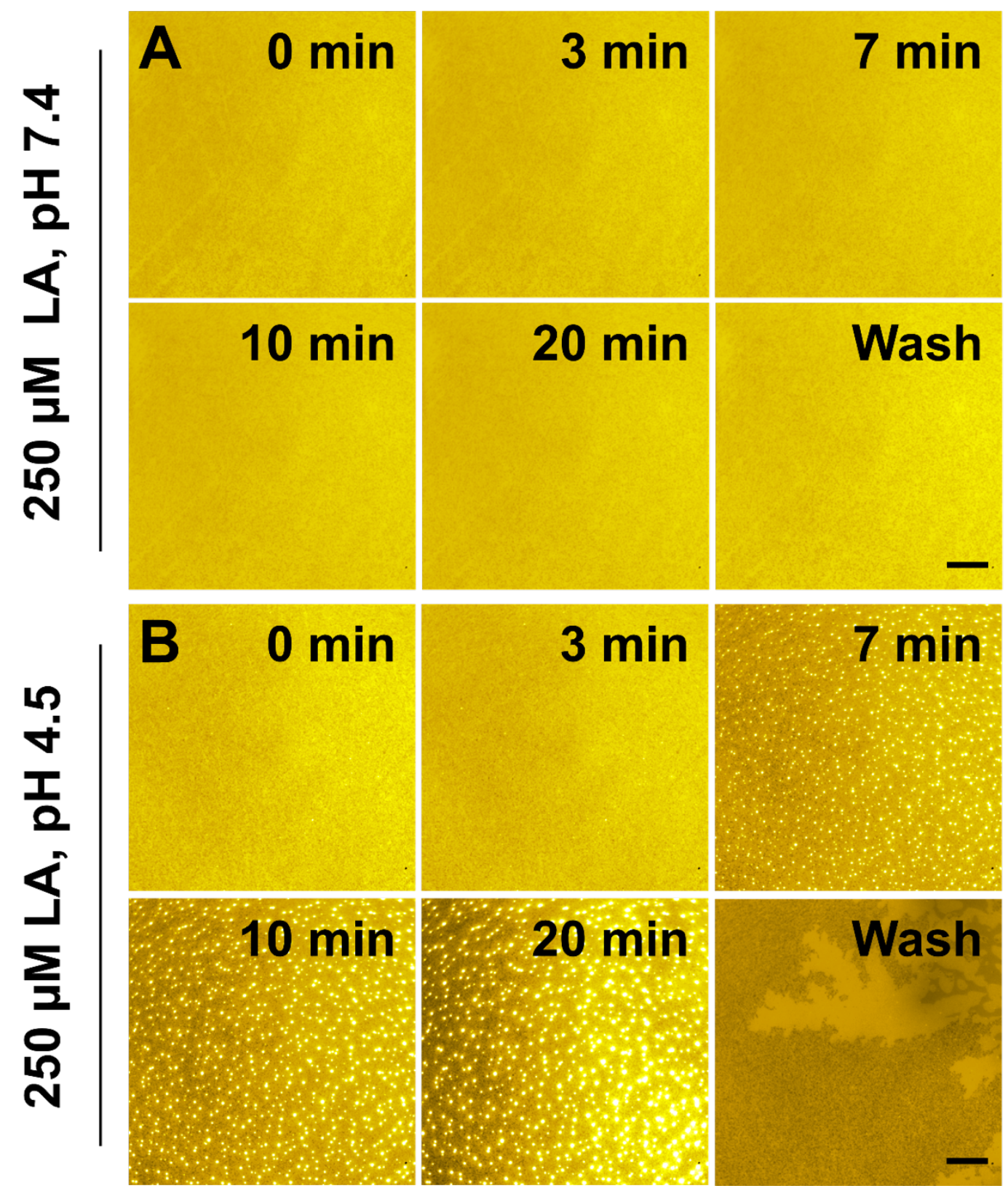

Figure 4.10. Microscopic observation of $250 \mu M$ lauric acid-induced membrane morphological responses on supported lipid bilayers. (A) Image snapshots at various time points depict the lack of membrane morphological response upon LA treatment of SLB at $\mathrm{pH}$ 7.4. (B) Image snapshots at various time points depict nucleation sites from which buds grow and membrane phase separation occurs upon LA treatment of SLB at $\mathrm{pH} 4.5$. $t=0 \mathrm{~min}$ corresponds to the introduction of $250 \mu \mathrm{M}$ LA solution into the measurement chamber. The scale bar is $20 \mu \mathrm{m}$. 


\section{$500 \mu M$ Glycerol Monolaurate}

We also investigated the effect of $500 \mu \mathrm{M}$ GML treatment on SLB platforms at both tested $\mathrm{pH}$ conditions. As shown in Figure 4.11A, GML treatment in the $\mathrm{pH} 7.4$ condition induced membrane budding, and the time-lapsed micrographs indicate that some tubules formed initially and became entangled, giving rise to the buds. These observations agree well with past results, ${ }^{14}$ and it was further seen that, upon buffer washing, GML treatment caused some degree of membrane lysis. On the other hand, Figure 4.11B shows that GML treatment at $\mathrm{pH} 4.5$ induced extensive membrane budding, and the buds coalesced to form larger bud-like structures, likely as a means of relieving membrane tension. Upon buffer washing, the large structures were removed while the underlying bilayer remained intact with nearly uniform fluorescence intensity. Some bright spots appeared on the bilayer surface, and likely correspond to nucleation sites. As such, GML treatment induced the formation of entangled tubules and/or buds in both cases, although the extent of membrane remodelling depended on the solution $\mathrm{pH}$. The observed trend agrees well with the QCM$\mathrm{D}$ measurement results, and suggests that the extent of possible membrane remodelling is greater at $\mathrm{pH} 4.5$ while the concentration-dependent activity profile of GML is equivalent across the tested $\mathrm{pH}$ conditions. 


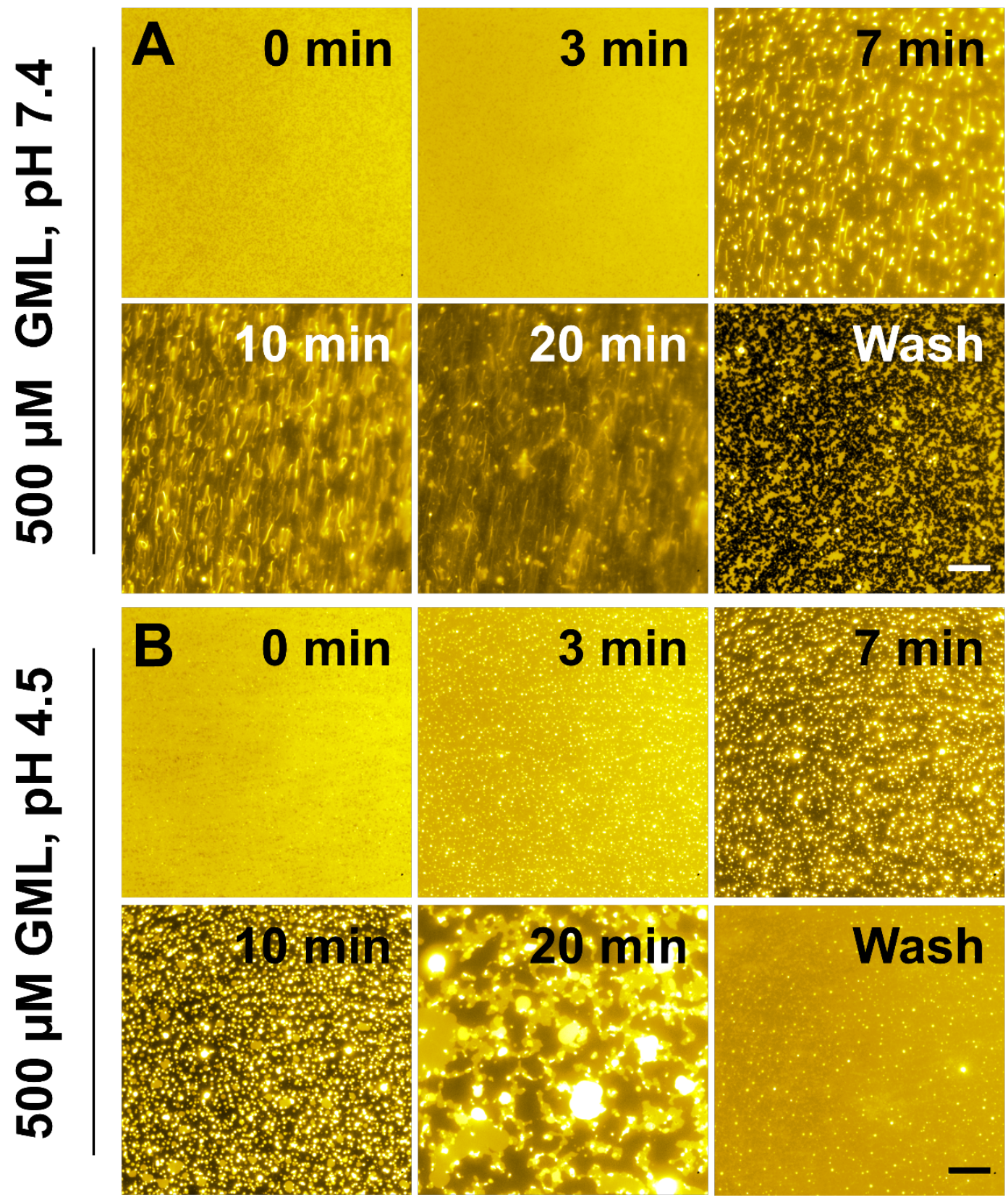

Figure 4.11. Microscopic observation of $500 \mu \mathrm{M}$ glycerol monolaurate-induced membrane morphological responses on supported lipid bilayers. (A) Image snapshots at various time points depict nucleation sites from which entangled tubes form, causing membrane budding and membrane lysis, upon GML treatment of SLB at pH 7.4. (B) Image snapshots at various time points depict nucleation sites from which buds grow upon GML treatment of SLB at $\mathrm{pH}$ 4.5. $t=0 \mathrm{~min}$ corresponds to the introduction of $500 \mu \mathrm{M}$ GML solution into the measurement chamber. The scale bar is $20 \mu \mathrm{m}$. 


\subsubsection{Evaluation of Antibacterial Activity}

The aforementioned results established that LA causes distinct types of membrane morphological changes, namely tubule formation or membrane phase separation, depending on the $\mathrm{pH}$ condition. On the other hand, GML caused membrane budding in all tested cases. These results motivated to further explore how $\mathrm{pH}$ conditions might affect the compounds' antibacterial activities, which stem from their membrane-disruptive activities. To address this question, it was investigated whether LA and GML can inhibit C. acnes, which is a Gram-positive bacteria that is a leading cause of bacterial skin infections, in different $\mathrm{pH}$ conditions. After incubating different concentrations of LA or GML with $C$. acnes bacteria $\left(1 \times 10^{6} \mathrm{CFU} / \mathrm{mL}\right)$ in the appropriate $\mathrm{pH}$ condition for $1 \mathrm{hr}$, the treated bacteria were streaked onto agar plates to facilitate bacterial growth under anaerobic conditions. Minimum inhibitory concentration (MIC) values were identified as the lowest concentration of LA or GML at which no visible growth of bacterial colonies was observed.

At $\mathrm{pH} 7.4$, it was determined that the MIC values of LA and GML were $1 \mathrm{mM}$ and 125 $\mu \mathrm{M}$, respectively (Figure 4.12.). The results agree with the range of reported MIC values of the two compounds against other Gram-positive bacteria, ${ }^{14,31}$ and indicate that both compounds inhibit $C$. acnes bacterial growth. Both compounds were also active only above their corresponding CMC values, which agrees well with the SLB experiments. While LA is known to inhibit $C$. acnes under neutral $\mathrm{pH}$ conditions, ${ }^{31}$ this is the first demonstration of GML inhibiting $C$. acnes. This finding is significant because, among antimicrobial lipids, LA is regarded as the most potent compound against $C$. acnes. As such, these results identify that GML is more potent than LA against C. acnes, supporting that GML is a promising antibacterial agent. 


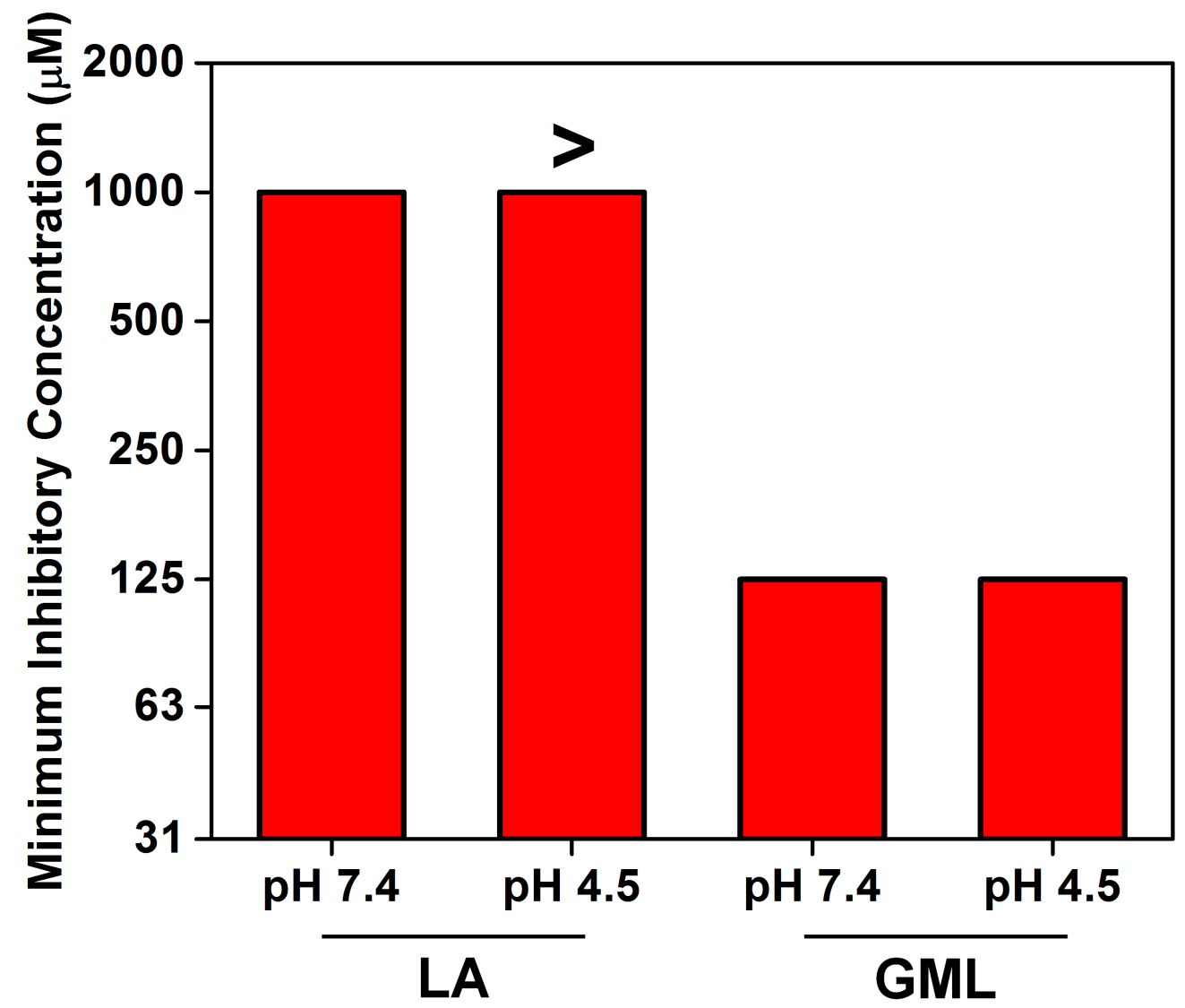

Figure 4.12. MIC values for antimicrobial lipids against $C$. acnes bacteria. The MIC values for LA and GML at pH 7.4 and 4.5 are reported from a minimum of three independent experiments using a two-fold dilution protocol. In the case of LA at $\mathrm{pH} 4.5$, the compound was inactive against C. acnes. A concentration higher than $1000 \mu \mathrm{M}$ is required (>). Standard deviations are negligible due to the assay format. The $y$-axis is presented in $\log _{2}$ scale for trend comparison.

In addition, the results show that GML had an MIC value of $125 \mu \mathrm{M}$ at $\mathrm{pH} 4.5$. This result is consistent with the foregoing experimental observations that GML had equivalent CMC values and exhibited a similar type of membrane-disruptive activity against SLB platforms at $\mathrm{pH} 4.5$ and 7.4. By contrast, LA was inactive against $C$. acnes at $\mathrm{pH} 4.5$. As discussed above, the SLB experiments revealed that LA causes membrane phase separation at $\mathrm{pH} 4.5$ in a distinct way that contrasts with how LA induces tubule formation in lipid bilayers at $\mathrm{pH}$ 7.4. While membrane phase separation caused by antibacterial compounds can inhibit bacterial growth in some cases, Gram-negative bacteria are more susceptible than Grampositive bacteria. ${ }^{52,53}$ This tendency explains why we observe that LA is inactive against 
Gram-positive $C$. acnes at $\mathrm{pH} 4.5$, while it has been previously reported that LA is active against Gram-negative Helicobacter pylori and Escherichia coli under acidic pH conditions. $^{54-56}$ Taken together, the MIC experiments demonstrate that GML exhibits inhibitory activity against $C$. acnes at $\mathrm{pH} 4.5$ and 7.4, and the similar levels of its antibacterial potency are consistent with the biophysical results. It was also determined that LA inhibits $C$. acnes at $\mathrm{pH} 7.4$ but is inactive at $\mathrm{pH}$ 4.5. This finding is also consistent with the biophysical results, which indicated that LA causes tubule formation or membrane phase separation in SLB platforms depending on the $\mathrm{pH}$ condition. When viewed collectively, the biophysical and biological results demonstrate that, and provide mechanistic insights to explain why, GML is a more promising candidate than LA to treat C. acnes infections.

\subsection{Conclusion}

In this chapter SLB platforms were employed in order to understand how LA and GML interact with lipid bilayers in acidic $\mathrm{pH}$ conditions, and compare the results with those obtained in near-neutral $\mathrm{pH}$ conditions. Two $\mathrm{pH}$ conditions were selected for testing, $\mathrm{pH}$ 4.5 and 7.4, which correspond to skin-like and physiological (blood) environments, respectively. While the CMC of LA was $900 \mu \mathrm{M}$ at $\mathrm{pH} 7.4$, it decreased to $100 \mu \mathrm{M}$ at $\mathrm{pH}$ 4.5 and this finding is consistent with the $\mathrm{pK}_{\mathrm{a}}$ value of LA, which was determined to be around 5.8. Specifically, the decrease in CMC occurs because the majority of LA molecules are protonated under acidic conditions and hence micellar aggregation becomes more thermodynamically favourable. On the other hand, the CMC of GML was $60 \mu \mathrm{M}$ in both cases, and the observed lack of $\mathrm{pH}$ dependence is consistent with its nonionic character. Using the QCM-D and time-lapsed fluorescence microscopy techniques, it was discovered that LA causes membrane phase separation in lipid bilayers under the $\mathrm{pH} 4.5$ condition. This membrane interaction was strikingly different from the tubule formation that is observed when LA interacts with lipid bilayers at $\mathrm{pH}$ 7.4. The trend in minimum concentrations of LA that was required for membrane-disruptive activity in the SLB platform was also in excellent agreement with the measured CMC values. Likewise, GML induced membrane budding in both tested $\mathrm{pH}$ conditions, with a similar GML- 
concentration-dependent profile that correlates with the unchanged $\mathrm{CMC}$ value. Of note, the extent of GML-induced membrane budding varied depending on the solution $\mathrm{pH}$, offering insight into how $\mathrm{pH}$-dependent bilayer mechanical properties also influence the membrane remodelling process. Collectively, the results demonstrate that solution $\mathrm{pH}$ can modulate the activity profile of ionizable antimicrobial lipids, and support that the ionization state of an antimicrobial lipid is an important determinant of the scope and potency of resulting membrane morphological responses.

While these results offer deep insights into the physical chemistry of antimicrobial lipids, they also have practical relevance for understanding related biological activities. In particular, antibacterial testing demonstrated that GML is active against $C$. acnes at both tested $\mathrm{pH}$ conditions, whereas LA is inhibitory only at $\mathrm{pH}$ 7.4. As a result, it was demonstrated that GML is 8-times more potent than LA and can function across a wider range of environmental conditions. These capabilities are directly linked to the physical chemistry of antimicrobial lipids, offering further motivation to continue developing integrative studies spanning biophysical and biological measurement approaches. Such information can provide guidance not only for utilizing these compounds to treat bacterial skin infections but also for developing formulations with suitable properties to achieve optimal performance when using antimicrobial lipids for a range of applications. 


\section{References}

1 Mukai, M. \& Regen, S. L. Lipid Raft Formation Driven by Push and Pull Forces. Bulletin of the Chemical Society of Japan 90, 1083-1087 (2017).

2 Denisov, I. G. \& Sligar, S. G. Nanodiscs in membrane biochemistry and biophysics. Chemical reviews 117, 4669-4713 (2017).

3 Komiyama, M., Yoshimoto, K., Sisido, M. \& Ariga, K. Chemistry can make strict and fuzzy controls for bio-systems: DNA nanoarchitectonics and cell-macromolecular nanoarchitectonics. Bulletin of the Chemical Society of Japan 90, 967-1004 (2017).

4 Kroll, A. V., Fang, R. H. \& Zhang, L. Biointerfacing and applications of cell membrane-coated nanoparticles. Bioconjugate chemistry 28, 23-32 (2016).

5 Desbois, A. P. \& Smith, V. J. Antibacterial free fatty acids: activities, mechanisms of action and biotechnological potential. Applied microbiology and biotechnology 85, 1629-1642 (2010).

6 Thormar, H. Lipids and Essential Oils as Antimicrobial Agents. (John Wiley \& Sons, 2011).

7 Thormar, H. \& Hilmarsson, H. The role of microbicidal lipids in host defense against pathogens and their potential as therapeutic agents. Chemistry and Physics of Lipids 150, 1-11 (2007).

8 Yoon, B. K., Jackman, J. A., Valle-González, E. R. \& Cho, N.-J. Antibacterial Free Fatty Acids and Monoglycerides: Biological Activities, Experimental Testing, and Therapeutic Applications. International Journal of Molecular Sciences 19, 1114 (2018).

9 Drake, D. R., Brogden, K. A., Dawson, D. V. \& Wertz, P. W. Thematic review series: skin lipids. Antimicrobial lipids at the skin surface. Journal of lipid research 49, 411 (2008).

10 Fischer, C. L. et al. The roles of cutaneous lipids in host defense. Biochimica et Biophysica Acta (BBA)-Molecular and Cell Biology of Lipids 1841, 319-322 (2014).

11 Chen, Y. E. \& Tsao, H. The skin microbiome: current perspectives and future challenges. Journal of the American Academy of Dermatology 69, 143-155. e143 (2013). 
12 Bergsson, G., Steingrímsson, Ó. \& Thormar, H. Bactericidal effects of fatty acids and monoglycerides on Helicobacter pylori. International Journal of Antimicrobial Agents 20, 258-262 (2002).

13 Kabara, J. J., Swieczkowski, D. M., Conley, A. J. \& Truant, J. P. Fatty acids and derivatives as antimicrobial agents. Antimicrobial agents and chemotherapy 2, 23-28 (1972).

14 Yoon, B. K., Jackman, J. A., Kim, M. C. \& Cho, N.-J. Spectrum of membrane morphological responses to antibacterial fatty acids and related surfactants. Langmuir 31, 10223-10232 (2015).

15 Kabara, J. J. Antimicrobial agents derived from fatty acids. Journal of the American Oil Chemists' Society 61, 397-403 (1984).

16 Conley, A. J. \& Kabara, J. J. Antimicrobial action of esters of polyhydric alcohols. Antimicrobial agents and chemotherapy 4, 501-506 (1973).

17 Kabara, J. J. Structure-function relationships of surfactants as antimicrobial agents. J Soc Cosmet Chem 29, 733-741 (1978).

18 Bergsson, G., Arnfinnsson, J., SteingrÍmsson, Ó. \& Thormar, H. Killing of Grampositive cocci by fatty acids and monoglycerides. APMIS 109, 670-678 (2001).

19 Bergsson, G., Arnfinnsson, J., Steingrímsson, Ó. \& Thormar, H. In vitro killing of Candida albicans by fatty acids and monoglycerides. Antimicrobial agents and chemotherapy 45, 3209-3212 (2001).

20 Skřivanová, E., Marounek, M., Dlouha, G. \& Kaňka, J. Susceptibility of Clostridium perfringens to $\mathrm{C} 2-\mathrm{C} 18$ fatty acids. Letters in applied microbiology 41, 77-81 (2005).

21 Hyldgaard, M., Sutherland, D. S., Sundh, M., Mygind, T. \& Meyer, R. L. Antimicrobial mechanism of monocaprylate. Applied and Environmental Microbiology 78, 2957-2965 (2012).

22 Thid, D., Benkoski, J. J., Svedhem, S., Kasemo, B. \& Gold, J. DHA-induced changes of supported lipid membrane morphology. Langmuir 23, 5878-5881 (2007).

23 Heerklotz, H. Interactions of surfactants with lipid membranes. Quarterly reviews of biophysics 41, 205-264 (2008). 
24 Staykova, M., Arroyo, M., Rahimi, M. \& Stone, H. A. Confined bilayers passively regulate shape and stress. Physical Review Letters 110, 028101 (2013).

25 Yoon, B. K., Jackman, J. A., Kim, M. C., Sut, T. N. \& Cho, N.-J. Correlating Membrane Morphological Responses with Micellar Aggregation Behavior of Capric Acid and Monocaprin. Langmuir 33, 2750-2759 (2017).

26 Lambers, H., Piessens, S., Bloem, A., Pronk, H. \& Finkel, P. Natural skin surface $\mathrm{pH}$ is on average below 5 , which is beneficial for its resident flora. International journal of cosmetic science 28, 359-370 (2006).

27 Pornpattananangkul, D. et al. In vivo treatment of Propionibacterium acnes infection with liposomal lauric acids. Advanced healthcare materials 2, 1322-1328 (2013). 28 Flanagan, J. L. et al. Glycerol Monolaurate Inhibits Lipase Production by Clinical Ocular Isolates Without Affecting Bacterial Cell ViabilityGlycerol Monolaurate Inhibits Bacterial Lipase Production. Investigative ophthalmology \& visual science 57, 544-550 (2016).

29 Mueller, E. A. \& Schlievert, P. M. Non-aqueous glycerol monolaurate gel exhibits antibacterial and anti-biofilm activity against Gram-positive and Gram-negative pathogens. PloS one 10, e0120280 (2015).

30 Hong Lioe Ko, S., Heczko, P. B. \& Pulverer, G. Differential susceptibility of Propionibacterium acnes, P. granulosum and P. avidum to free fatty acids. Journal of Investigative Dermatology 71 (1978).

31 Nakatsuji, T. et al. Antimicrobial property of lauric acid against Propionibacterium acnes: its therapeutic potential for inflammatory acne vulgaris. Journal of investigative dermatology 129, 2480-2488 (2009).

32 Yang, D. et al. The antimicrobial activity of liposomal lauric acids against Propionibacterium acnes. Biomaterials 30, 6035-6040 (2009).

33 Huang, W.-C. et al. Anti-bacterial and anti-inflammatory properties of capric acid against Propionibacterium acnes: a comparative study with lauric acid. Journal of dermatological science 73, 232-240 (2014).

34 Rodahl, M., Höök, F., Krozer, A., Brzezinski, P. \& Kasemo, B. Quartz crystal microbalance setup for frequency and Q-factor measurements in gaseous and liquid environments. Review of Scientific Instruments 66, 3924-3930 (1995). 
35 Lood, R. \& Collin, M. Characterization and genome sequencing of two Propionibacterium acnes phages displaying pseudolysogeny. BMC genomics 12, 198 (2011).

36 Galbraith, H. \& Miller, T. Effect of long chain fatty acids on bacterial respiration and amino acid uptake. Journal of Applied Microbiology 36, 659-675 (1973).

37 Zhou, X., Zeitz, J., Meile, L., Kreuzer, M. \& Schwarm, A. Influence of pH and the degree of protonation on the inhibitory effect of fatty acids in the ruminal methanogen Methanobrevibacter ruminantium strain M1. Journal of applied microbiology 119, 14821493 (2015).

38 Jensen, M. G. (Department of Biomedicine, Health Aarhus University, Denmark, 2016).

39 Ananthapadmanabhan, K., Goddard, E., Turro, N. \& Kuo, P. Fluorescence probes for critical micelle concentration. Langmuir 1, 352-355 (1985).

40 Kanicky, J. \& Shah, D. Effect of premicellar aggregation on the $\mathrm{pK}_{\mathrm{a}}$ of fatty acid soap solutions. Langmuir 19, 2034-2038 (2003).

41 Goodman, D. S. The distribution of fatty acids between n-heptane and aqueous phosphate buffer. Journal of the American Chemical Society 80, 3887-3892 (1958).

42 Spector, A. A. Fatty acid binding to plasma albumin. Journal of lipid research 16, 165-179 (1975).

43 Small, D. in Nestle nutrition workshop series (USA).

44 Tabaei, S. R., Choi, J.-H., Haw Zan, G., Zhdanov, V. P. \& Cho, N.-J. Solventassisted lipid bilayer formation on silicon dioxide and gold. Langmuir 30, 10363-10373 (2014).

45 Tabaei, S. R., Jackman, J. A., Kim, S.-O., Zhdanov, V. P. \& Cho, N.-J. Solventassisted lipid self-assembly at hydrophilic surfaces: factors influencing the formation of supported membranes. Langmuir 31, 3125-3134 (2015).

46 Kawakami, L. M. et al. Understanding How Sterols Regulate Membrane Remodeling in Supported Lipid Bilayers. Langmuir (2017).

47 Jackman, J. A., Cho, N.-J., Duran, R. S. \& Frank, C. W. Interfacial binding dynamics of bee venom phospholipase $A_{2}$ investigated by dynamic light scattering and quartz crystal microbalance. Langmuir 26, 4103-4112 (2009). 
48 Zhou, Y. \& Raphael, R. M. Solution $\mathrm{pH}$ alters mechanical and electrical properties of phosphatidylcholine membranes: relation between interfacial electrostatics, intramembrane potential, and bending elasticity. Biophysical Journal 92, 2451-2462 (2007).

49 Boggara, M. B., Faraone, A. \& Krishnamoorti, R. Effect of pH and ibuprofen on the phospholipid bilayer bending modulus. The Journal of Physical Chemistry B 114, 8061-8066 (2010).

50 Tabaei, S. R., Jackman, J. A., Liedberg, B., Parikh, A. N. \& Cho, N.-J. Observation

of Stripe Superstructure in the $\beta$-Two-Phase Coexistence Region of CholesterolPhospholipid Mixtures in Supported Membranes. Journal of the American Chemical Society 136, 16962-16965 (2014).

51 Seul, M. \& Andelman, D. Domain shapes and patterns: the phenomenology of modulated phases. Science 267, 476 (1995).

52 Epand, R. M. \& Epand, R. F. Lipid domains in bacterial membranes and the action of antimicrobial agents. Biochimica et Biophysica Acta (BBA)-Biomembranes 1788, 289294 (2009).

53 Epand, R. M. \& Epand, R. F. Bacterial membrane lipids in the action of antimicrobial agents. Journal of Peptide Science 17, 298-305 (2011).

54 Yang, J., Hou, X., Mir, P. S. \& McAllister, T. A. Anti-Escherichia coli O157: H7 activity of free fatty acids under varying pH. Canadian Journal of Microbiology 56, 263267 (2010).

55 Sun, C. Q., O'Connor, C. J. \& Roberton, A. M. Antibacterial actions of fatty acids and monoglycerides against Helicobacter pylori. FEMS Immunology \& Medical Microbiology 36, 9-17 (2003).

56 Petschow, B. W., Batema, R. P. \& Ford, L. L. Susceptibility of Helicobacter pylori to bactericidal properties of medium-chain monoglycerides and free fatty acids. Antimicrobial Agents and Chemotherapy 40, 302-306 (1996). 


\section{Chapter 5}

\section{pH-Dependent Antibacterial Activity of Glycolic Acid: Implications for Anti-Acne Formulations*}

Glycolic acid is the smallest alpha hydroxy acid and widely used for skincare applications, including to treat acne vulgaris. Oftentimes, high concentrations of glycolic acid ( 20-50 vol\%) are incorporated into chemical peels to reduce acne-related inflammation while there is an outstanding need to determine to what extent glycolic acid can potently inhibit Cutibacterium acnes (formerly known as Propionibacterium acnes), which is a Gram-positive bacterium implicated in acne pathogenesis. This chapter reports that glycolic acid exhibits $\mathrm{pH}$ dependent antibacterial activity against C. acnes and mechanistic studies identified that the nonionic form of glycolic acid is more active than the anionic form. The degree of antibacterial activity, including minimum bactericidal concentration (MBC), of glycolic acid was evaluated in the $\mathrm{pH}$ range of 3 to 4.5 , and the greatest potency was observed at $\mathrm{pH}$ 3. In light of skincare formulation needs, the $\mathrm{pH} 3.5$ condition was selected for further testing and determined that glycolic acid kills C. acnes cells by disrupting bacterial cell membranes. While most conventional treatments involve high concentrations of glycolic acid (>20\%), findings support the potential of developing anti-acne formulations with glycolic acid concentrations as low as $0.2 \%$ and with pH conditions that are suitable for over-the-counter applications.

*This chapter is published substantially as Valle-González, E. R., Jackman, J. A., Yoon, B. K., Mokrzecka, N. \& Cho, N.-J. pH Dependent Antibacterial Activity of Glycolic Acid: Implications for Anti-Acne Formulations. Scientific Reports 10, 7491 (2020). DOI: 10.1038/s41598-020-64545-9. Reproduced with permission, Copyright (2020) Springer Nature. 


\subsection{Introduction}

Acne vulgaris is a chronic inflammatory disease of hair follicles that causes cosmetically unfavorable lesions on the skin surface, which makes it a leading dermatological problem worldwide ${ }^{1,2}$. The origin of acne vulgaris is multifaceted and involves a few key steps ${ }^{3}$. First, the excessive production of oily secretions from hair follicles can cause proliferation of pathogenic Cutibacterium acnes (Propionibacterium acnes) bacterial strains on the skin surface and in the follicles ${ }^{4,5}$. In some cases, this bacterial overgrowth and accompanying changes in skin microflora can induce hyper keratinization and inflammation, which triggers the formation of skin lesions ${ }^{6}$. There are many strategies to treat acne vulgaris and one promising approach involves using antibiotics to inhibit C. acnes on the skin surface ${ }^{7}$. However, antibiotic treatments can have drawbacks such as skin irritation and the emergence of antibiotic-resistant $C$. acnes strains ${ }^{8-10}$. These issues have led to the exploration of natural antibacterial solutions ${ }^{11}$ such as membrane-disruptive antimicrobial fatty acids that can inhibit $C$. acnes while posing lower risks for resistance development ${ }^{12-}$ 14. Such strategies have led to the growing rise in topical dermocosmetics to treat acne vulgaris ${ }^{15,16}$.

Within this scope, glycolic acid - an important alpha hydroxy acid - merits attention because it is one of the most widely used natural compounds in the skincare industry and is readily extracted from fruit juices and sugar cane ${ }^{17}$. Glycolic acid is a key component of aqueous solutions used in chemical peeling procedures, in which case the outermost layer of the skin surface is exfoliated in order to rejuvenate the skin by reducing scarring and inflammation ${ }^{18}$. In patients with acne vulgaris, glycolic acid treatment can lead to significant reductions in the number of skin lesions ${ }^{19}$. Typically, high glycolic acid concentrations ( $>30 \mathrm{vol} \%$ ) are used for skin exfoliation and pore unclogging while lower concentrations $(<15 \mathrm{vol} \%)$ are used to prevent pore occlusion ${ }^{20}$. Thus, chemical peeling procedures based on glycolic acid are currently used to treat acne vulgaris as an adjuvant treatment $^{21,22}$. There is also some evidence that glycolic acid might reduce hyper keratinization as well ${ }^{23}$. Interestingly, Takenaka et al. reported that 30-35 vol\% glycolic acid exhibits antibacterial activity against $C$. acnes and can decrease $C$. acnes concentrations on the checks of acne vulgaris patients in a human clinical trial ${ }^{24}$. Notably, 
the chemical peel used in that study contained $35 \mathrm{vol} \%$ glycolic acid at $\mathrm{pH} 1.2$, which is not suitable for over-the-counter topical use ${ }^{25}$. Current guidance supported by the US Food and Drug Administration advises that glycolic acid formulations in dermocosmetic products be within the range of $\leq 10$ vol\% glycolic acid concentration and formulation $\mathrm{pH}$ $\geq 3.5$. Therefore, it would be advantageous to further explore the antibacterial properties of glycolic acid in order to devise more broadly useful treatment strategies that are suitable for over-the-counter application usage.

Herein, it was investigated the concentration-dependent antibacterial activity of glycolic acid against $C$. acnes in different $\mathrm{pH}$ conditions and identified that glycolic acid inhibits $C$. acnes at $>100$-fold lower concentrations than previously reported (down to $\sim 0.2 \%$ glycolic acid at $\mathrm{pH} 3.5$ ). The $\mathrm{pH}$-dependent range of antibacterial activity to inhibit bacterial cell viability was tested, followed by determining the minimum bactericidal concentration (MBC) values of glycolic acid at specific $\mathrm{pH}$ conditions. It was identified that glycolic acid potently inhibits $C$. acnes in the $\mathrm{pH}$ range of 3-4.5. Within this $\mathrm{pH}$ range, glycolic acid had greater potency at lower $\mathrm{pH}$ when more glycolic acid molecules were in the nonionic form. Mechanistic studies further supported that glycolic acid is bactericidal and disrupts $C$. acnes cell membrane integrity. Taken together, the findings support that glycolic acid inhibits $C$. acnes bacteria and is thus a promising agent to treat acnes vulgaris, especially since its mechanism of action could potentially offer a higher barrier to resistance development as compared to currently used antibiotics.

\subsection{Materials and Methods}

\subsubsection{Materials}

Glycolic acid and sodium hydroxide were procured from Sigma-Aldrich (St. Louis, MO). Tryptic soy broth (TSB) and the BD GasPak EZ Incubation Container System, including sachets, were obtained from Becton Dickinson (Franklin Lakes, NJ). Defibrinated sheep blood was supplied by Thermo Fisher Scientific (Waltham, MA). Tryptic soy agar plates with 5\% defibrinated sheep blood were acquired from Hardy Diagnostics (Santa Maria, CA). Phosphate-buffered saline (PBS) was procured from Gibco (Carlsbad, CA). The 
Live/Dead BacLight Bacterial Viability Kit was obtained from Invitrogen/Molecular Probes (Carlsbad, CA). The ATP Bioluminescence Assay Kit HS II was procured from Roche (Mannheim, Germany). All solutions were prepared with Milli-Q-treated deionized water (>18 M $\Omega \cdot \mathrm{cm}$ resistivity) (MilliporeSigma, Burlington, MA).

\subsubsection{Glycolic Acid Preparation}

Stock solutions of $200 \mathrm{mM}$ glycolic acid were prepared in PBS. The initial solution $\mathrm{pH}$ was around 2.3 and the $\mathrm{pH}$ was adjusted by adding $3 \mathrm{M} \mathrm{NaOH}$, followed by stirring and $\mathrm{pH}$ monitoring using an electronic $\mathrm{pH}$ meter (Accumet AB15, Thermo Fisher Scientific; Waltham, MA). The procedure was repeated until reaching the desired $\mathrm{pH}$ value. Before experiment, glycolic acid samples were heated at $55^{\circ} \mathrm{C}$ for $30 \mathrm{~min}$, and then cooled down before measurements were conducted at room temperature. The solution $\mathrm{pH}$ was rechecked immediately before experiment.

\subsubsection{Bacterial Cell Culture}

A quality control strain of C. acnes (ATCC 11827, American Type Culture Collection, Manassas, VA) was cultured in Tryptic soy broth with 5\% defibrinated sheep blood for 48 hrs under anaerobic conditions using a Gas-Pak $\left(80 \% \mathrm{~N}_{2}, 13 \% \mathrm{CO}_{2}, 7 \% \mathrm{H}_{2}\right)$ at $37{ }^{\circ} \mathrm{C}$. The bacterial suspension was then re-inoculated in fresh Tryptic soy broth with 5\% defibrinated sheep blood and incubated under the same anaerobic conditions for an additional $24 \mathrm{hrs}$. The bacterial cells were next harvested by centrifugation at $1485 \times \mathrm{g}$ for $10 \mathrm{~min}$, washed thrice with PBS, and re-suspended in the same buffer. The $\mathrm{OD}_{600}$ value was measured and the appropriate dilution was made to reach a value of $\sim 0.35$ (mid-exponential growth phase $)^{33}$. This value corresponds to a density of $2 \times 10^{8} \mathrm{CFU} \mathrm{mL}^{-1}$, as confirmed by colonyforming unit (CFU) enumeration in control experiments. The bacteria suspension was diluted with PBS to a density of $2 \times 10^{6} \mathrm{CFU} \mathrm{mL} \mathrm{m}^{-1}$ for testing purposes. 


\subsubsection{Antibacterial Testing}

The antibacterial activity of glycolic acid samples was evaluated by incubating $C$. acnes suspensions in glycolic acid solutions, followed by agar plating to determine resulting $C$. acnes viability based on colony growth. At different $\mathrm{pH}$ values, glycolic acid samples were tested in the concentration range of $200 \mathrm{mM}$ to $1.6 \mathrm{mM}$ in a two-fold dilution series in a 96-well plate format. The glycolic acid samples were incubated with $C$. acnes at a concentration of $1 \times 10^{6} \mathrm{CFU} \mathrm{mL}^{-1}$ under anaerobic conditions for $1 \mathrm{hr}$ at $37{ }^{\circ} \mathrm{C}$ before spotting onto tryptic soy agar plates supplemented with 5\% defibrinated sheep blood. The streaked plates were then incubated under anaerobic conditions at $37^{\circ} \mathrm{C}$ for 4 days, after which the presence of bacterial colonies in each test group was evaluated. The reported inhibitory concentrations of glycolic acid in different $\mathrm{pH}$ conditions were determined by identifying the lowest concentration of glycolic acid that completely inhibited the visible growth of bacterial colonies upon agar plating. All experiments were performed in triplicate, including positive controls (bacteria incubated without glycolic acid at test $\mathrm{pH}$ conditions) and negative controls (solution without bacteria at test $\mathrm{pH}$ conditions).

\subsubsection{Minimum Bactericidal Concentration (MBC) Testing}

Glycolic acid samples were prepared at different $\mathrm{pH}$ values in the concentration range of $200 \mathrm{mM}$ to $1.6 \mathrm{mM}$ and incubated with $C$. acnes at a concentration of $1 \times 10^{6} \mathrm{CFU} \mathrm{mL}^{-1}$. The samples were cultured under anaerobic conditions for $1 \mathrm{hr}$ at $37^{\circ} \mathrm{C}$, and then each sample was diluted in a 10-fold series and streaked onto tryptic soy agar plates supplemented with 5\% defibrinated sheep blood. The streaked plates were incubated under anaerobic conditions at $37^{\circ} \mathrm{C}$ for 4 days, and the $\mathrm{CFU}$ density of each sample was next determined. The effect of glycolic acid concentration on cell viability was determined at each tested $\mathrm{pH}$ condition along with the corresponding $\mathrm{MBC}$ value, which was defined as the lowest concentration of glycolic acid that reduced bacterial cell viability by at least 99.99\%. All experiments were performed in triplicate, including positive controls (bacteria incubated without glycolic acid at test $\mathrm{pH}$ conditions) and negative controls (solution without bacteria at test $\mathrm{pH}$ conditions). 


\subsubsection{Live/Dead Bacterial Cell Staining}

Glycolic acid samples were prepared at $\mathrm{pH} 3.5$ in the concentration range of $50 \mathrm{mM}$ to 3.1 $\mathrm{mM}$ and then added to $C$. acnes suspensions at a final concentration of $1 \times 10^{8} \mathrm{CFU} \mathrm{mL}^{-1}$. The samples were incubated under anaerobic conditions for $1 \mathrm{hr}$ at $37^{\circ} \mathrm{C}$ and then stained with fluorescent dyes by using the Live/Dead BacLight Bacterial Viability Kit (Molecular Probes, Invitrogen, Carlsbad, CA) according to the manufacturer's protocol. The stained bacterial samples were then observed using an LSM 710 confocal laser scanning microscope (Zeiss, Oberkochen, Germany) along with appropriate negative and positive controls. Live and dead bacterial cells could be visualized by green and red stains, respectively.

\subsubsection{ATP Release Measurements}

C. acnes suspensions were diluted to an $\mathrm{OD}_{600}$ value of $\sim 0.5$ that corresponds to a density of $4 \times 10^{8} \mathrm{CFU} \mathrm{mL} \mathrm{m}^{-1}$. Glycolic acid samples were prepared at $\mathrm{pH} 3.5$ in the concentration range of $50 \mathrm{mM}$ to $12.5 \mathrm{mM}$ and then added to $C$. acnes suspensions at a final concentration of $2 \times 10^{8} \mathrm{CFU} \mathrm{mL} \mathrm{m}^{-1}$. The samples were incubated under anaerobic conditions for $1 \mathrm{hr}$ at $37^{\circ} \mathrm{C}$, followed by titrating the samples with $3 \mathrm{M} \mathrm{NaOH}$ to increase the $\mathrm{pH}$ value to $\sim 7.5$, which is suitable for bioluminescence measurements. Then, the samples were analyzed by the ATP Bioluminescence Assay Kit HS II (Roche), which is based on the luciferasecatalyzed oxidation of luciferin that emits light. The relative amount of bioluminescence was measured by using a Cytation 5 cell imaging multi-mode microplate reader (BioTek, Winooski, VT). The bioluminescent signals were converted into ATP concentrations based on a standard curvature.

\subsubsection{Statistical Analysis}

Data were analyzed with GraphPad Prism software (San Diego, CA) and compared by oneway analysis of variance (ANOVA) with Dunnett's multiple comparisons test (versus untreated control, indicated by *) in Figures 5.2 and 5.4 or by the unpaired student's t-test 
in Figure 5.3. The statistical significance was computed in terms of a multiplicity-adjusted $\mathrm{P}$ values, and $P<0.05, P<0.01$, and $P<0.001$ indicate the levels of statistical significance $(*, * *, * * *)$.

\subsection{Results and Discussion}

\subsubsection{Antibacterial potency as a function of solution $\mathrm{pH}$}

Glycolic acid is the smallest alpha hydroxy acid and consists of a carboxylic acid functional group along with a hydroxyl functional group at the neighboring $\alpha$-carbon position ${ }^{26}$ (Figure 5.1a). Only the carboxylic acid group is ionizable in biologically relevant $\mathrm{pH}$ conditions and glycolic acid exists in an equilibrium between two molecular states: (1) nonionic when the carboxylic acid is protonated at low $\mathrm{pH}$ conditions and (2) anionic when the carboxylic acid is deprotonated at high $\mathrm{pH}$ conditions. The acid disassociation constant, $\mathrm{pK}_{\mathrm{a}}$, is defined as the $\mathrm{pH}$ value at which half of the glycolic acid molecules are nonionic and the other half are anionic. It has been reported that the $\mathrm{pK}_{\mathrm{a}}$ value of glycolic acid is around pH 3.83 (Ref. 27). 


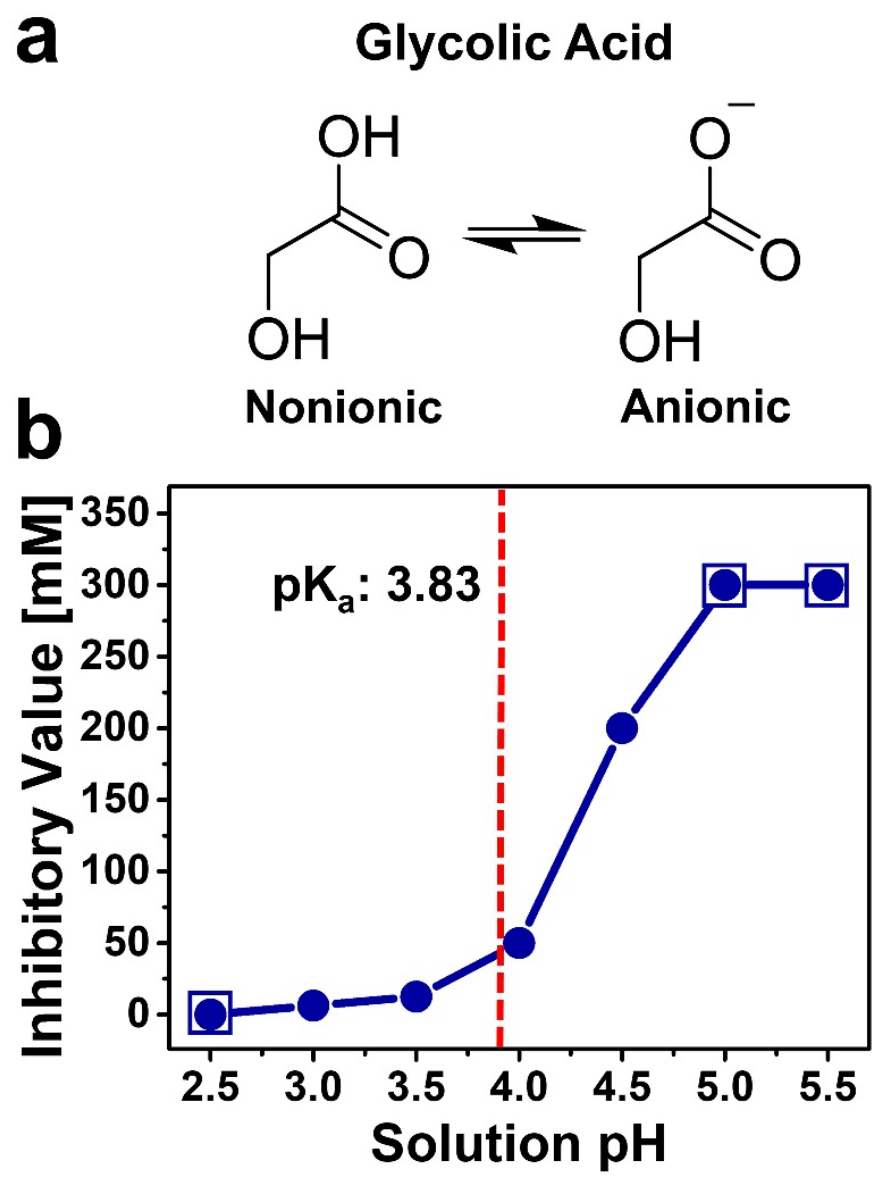

Figure 5.1. Evaluation of glycolic acid as an antibacterial agent to inhibit $C$. acnes viability. (a) Molecular structure of glycolic acid in the nonionic (protonated) and anionic (deprotonated) states. The equilibrium ratio of glycolic acid molecules in the two states depends on the $\mathrm{pH}$ condition. (b) Experimentally determined lowest concentration of glycolic acid to fully inhibit $C$. acnes viability in different $\mathrm{pH}$ conditions. The $C$. acnes cell concentration was $1 \times 10^{6} \mathrm{CFU} \mathrm{mL}^{-1}$ and cell suspensions were incubated in glycolic acid solutions at different $\mathrm{pH}$ conditions for $1 \mathrm{hr}$ before agar plating to determine if glycolic acid treatment inhibited cell viability. Each data point is representative of three independent experiments. The boxed-in circles indicate $\mathrm{pH}$ conditions where an inhibitory concentration was not recorded for one of two reasons: the $\mathrm{pH}$ condition itself during the incubation step caused loss of $C$. acnes viability ( $\mathrm{pH} 2.5$ ) or glycolic acid was inactive (i.e., not antibacterial) within the test range up to $200 \mathrm{mM}$ (pH 5.0 and 5.5). The dashed vertical line represents the $\mathrm{pK}_{\mathrm{a}}$ value of glycolic acid, which is around $\mathrm{pH} 3.83$.

Therefore, it was first evaluated the antibacterial activity of glycolic acid in different $\mathrm{pH}$ conditions. C. acnes suspensions were incubated for $1 \mathrm{hr}$ in two-fold-diluted sets of 
glycolic acid solutions (200 $\mathrm{mM}$ to $1.6 \mathrm{mM})$ and each set had been prepared at a specific $\mathrm{pH}$ value between 2.5 and 5.5, followed by agar plating to determine the lowest glycolic acid concentration at which no bacterial growth was visible due to antibacterial activity at the incubated $\mathrm{pH}$ condition (Figure 5.1b). At pH 2.5, the solution was too acidic to support subsequent $C$. acnes viability so no inhibitory concentration was recorded for glycolic acid in that case. By contrast, C. acnes was still viable after incubation at $\mathrm{pH} 3.0$ and the corresponding inhibitory concentration of glycolic acid at which bacterial growth was fully inhibited was $6.3 \mathrm{mM}$. At higher $\mathrm{pH}$ values, C. acnes remained viable after incubation and glycolic acid exhibited $\mathrm{pH}$-dependent antibacterial activity. The corresponding inhibitory concentrations of glycolic acid at $\mathrm{pH} 3.5,4.0$, and 4.5 were $12.5 \mathrm{mM}, 50 \mathrm{mM}$, and 200 $\mathrm{mM}$, respectively. At higher $\mathrm{pH}$ values of 5.0 and 5.5, glycolic acid did not exhibit antibacterial activity in the test range up to $200 \mathrm{mM}$. Together, the data support that glycolic acid exhibits greater antibacterial potency at lower $\mathrm{pH}$ values.

By application of the Henderson-Hasselbalch equation, these findings further support that glycolic acid has more potent antibacterial properties when it is present in its nonionic form. At $\mathrm{pH}$ values of 3.0 and 3.5, more than $87 \%$ and $68 \%$ of glycolic acid molecules are in the nonionic form, respectively. By contrast, at $\mathrm{pH}$ values of 4.0 and 4.5 , less than $41 \%$ and $18 \%$ of glycolic acid molecules are in the nonionic form, respectively.

\subsubsection{Characterization of bactericidal activity}

Next, colony-forming unit (CFU) enumeration assay experiments were conducted in order to evaluate the concentration range at which glycolic acid kills $C$. acnes (by at least 99.99\%) in different $\mathrm{pH}$ conditions, including determining the minimum bactericidal concentration $(\mathrm{MBC})$ values. At $\mathrm{pH} 2.5$, the solution was too acidic to promote $C$. acnes viability and thus no $\mathrm{MBC}$ value was recorded (Figure 5.2a). At $\mathrm{pH} 3.0$, there was C. acnes viability with a viable cell concentration around $1 \times 10^{6} \mathrm{CFU} \cdot \mathrm{mL}^{-1}$ and glycolic acid exhibited concentration-dependent bactericidal activity (Figure 5.2b). Treatment with 3.1 and $6.3 \mathrm{mM}$ glycolic acid significantly reduced the viable cell concentration to around $7 \times$ $10^{5} \mathrm{CFU} \cdot \mathrm{mL}^{-1}$ and $4 \times 10^{3} \mathrm{CFU} \cdot \mathrm{mL}^{-1}$, respectively. The $\mathrm{MBC}$ value recorded at $\mathrm{pH} 3.0$ 
was $12.5 \mathrm{mM}$. At pH 3.5, the bactericidal activity of glycolic acid was still significant but less potent (Figure 5.2c). There was a negligible reduction in cell viability upon treatment with $3.1 \mathrm{mM}$ glycolic acid, whereas treatment with 6.3 and $12.5 \mathrm{mM}$ glycolic acid significantly reduced the viable cell concentration from around $1 \times 10^{6} \mathrm{CFU} \cdot \mathrm{mL}^{-1}$ to $7 \times$ $10^{5} \mathrm{CFU} \cdot \mathrm{mL}^{-1}$ and $4 \times 10^{3} \mathrm{CFU} \cdot \mathrm{mL}^{-1}$, respectively. The $\mathrm{MBC}$ value recorded at $\mathrm{pH} 3.5$ was $25 \mathrm{mM}$. At pH 4.0, glycolic acid still exhibited bactericidal activity but it was even less potent (Figure 5.2d). Treatment with 3.1 and $6.3 \mathrm{mM}$ glycolic acid led to negligible reductions in cell viability. On the other hand, treatment with 12.5 and $25 \mathrm{mM}$ glycolic acid significantly reduced the viable cell concentration from around $1 \times 10^{6} \mathrm{CFU} \cdot \mathrm{mL}^{-1}$ to $4 \times 10^{5} \mathrm{CFU} \cdot \mathrm{mL}^{-1}$ and $6 \times 10^{3} \mathrm{CFU} \cdot \mathrm{mL}^{-1}$, respectively. The $\mathrm{MBC}$ value recorded at $\mathrm{pH}$ 4.0 was $50 \mathrm{mM}$. Together, the $\mathrm{MBC}$ data corresponded well with the aforementioned results and support that glycolic acid exhibits $\mathrm{pH}$-dependent antibacterial activity against C. acnes. 


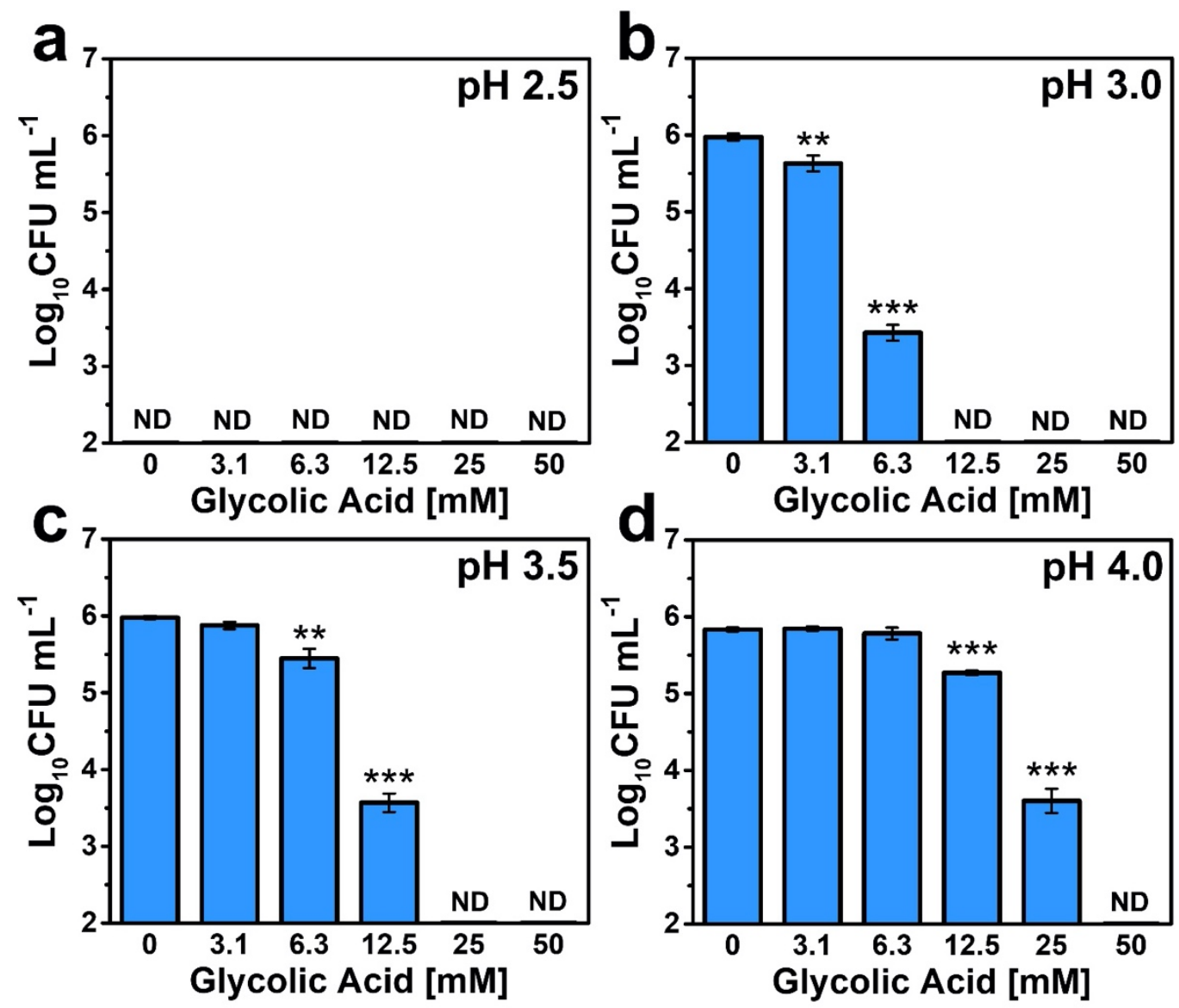

Figure 5.2. pH-dependent bactericidal activity of glycolic acid against $\boldsymbol{C}$. acnes. The $\mathrm{pH}$ dependent reduction in viable $C$. acnes cell concentration is plotted as a function of glycolic acid concentration. The data correspond to (a) $\mathrm{pH} 2.5$, (b) $\mathrm{pH} 3.0$, (c) $\mathrm{pH} 3.5$, and (d) $\mathrm{pH} 4.0$. The $C$. acnes cell concentration was $1 \times 10^{6} \mathrm{CFU} \mathrm{mL}^{-1}$. The recorded $\mathrm{MBC}$ values are $12.5 \mathrm{mM}, 25 \mathrm{mM}$, and $50 \mathrm{mM}$ at $\mathrm{pH} 3.0,3.5$, and 4.0, respectively, and correspond to the lowest glycolic acid concentration at which no bacterial cell viability was detected (reported as ND, not determined). $C$. acnes cells were not viable after incubation in the $\mathrm{pH} 2.5$ condition, whereas viability was maintained after incubation in the other test $\mathrm{pH}$ conditions as indicated by the $0 \mathrm{mM}$ glycolic acid control data for each case. Mean \pm standard deviation values are reported from $n=3$ experiments. 


\subsubsection{Microscopic observation of bacterial cell killing}

To confirm cell killing at $\mathrm{pH} 3.5$, confocal microscopy imaging was performed to distinguish live and dead $C$. acnes cells upon treatment with glycolic acid. A twofluorophore staining approach was used, whereby the SYTO 9 dye (green color) can translocate across all bacterial cell membranes while the PI dye (red color) can only permeate the cell membranes of dead bacterial cells with compromised membrane integrity $^{29}$. Using a CFU enumeration assay, it was first confirmed that glycolic acid decreases $C$. acnes cell viability at a higher cell density of $1 \times 10^{8} \mathrm{CFU} \mathrm{mL}^{-1}$ (100-fold higher than the antibacterial testing conditions described above and necessary for cell imaging purposes) (Figure 5.3a). 


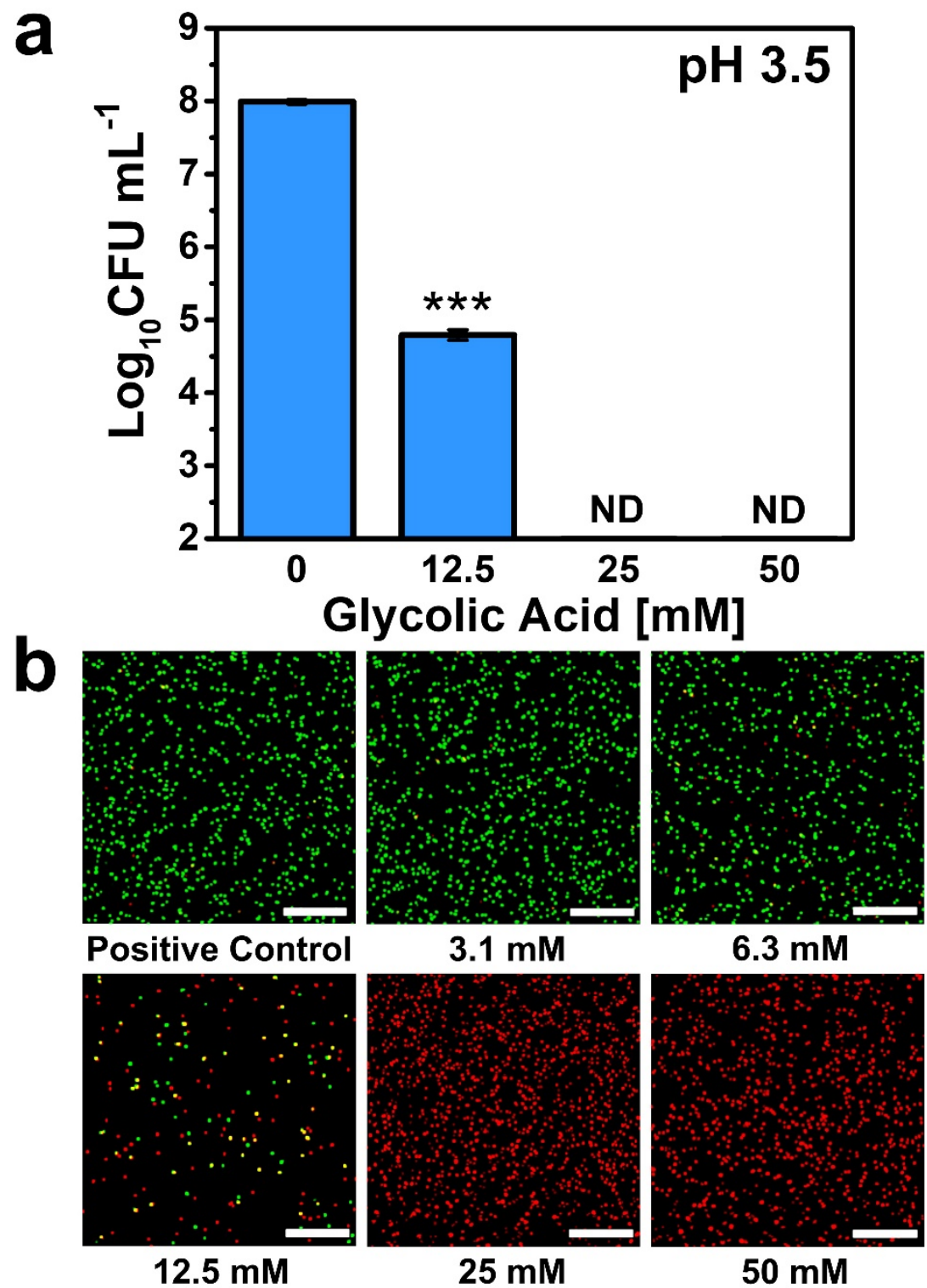

Figure 5.3. Effect of glycolic acid treatment on $C$. acnes cell viability. (a) Effect of glycolic acid concentration-dependent treatment on viable $C$. acnes cell concentration at $\mathrm{pH} 3.5$. The $C$. acnes cell concentration was $1 \times 10^{8} \mathrm{CFU} \mathrm{mL}^{-1}$. The recorded $\mathrm{MBC}$ value was $25 \mathrm{mM}$, and corresponds to the lowest glycolic acid concentration at which no bacterial cell viability was detected (reported as ND, not determined). Mean \pm standard deviation values are reported from $n=3$ experiments. (b) Live-dead assay evaluation of glycolic acid-treated C. acnes cells at different glycolic acid concentrations by confocal laser scanning microscopy (CLSM) imaging. Green and red colors indicate live and dead C. acnes cells, respectively. Images are representative of three experiments. Scale bars: $100 \mu \mathrm{m}$. 
The basic operating principles of the microscopy imaging protocol were also confirmed using a positive control (live; green color) sample consisting of untreated C. acnes cells incubated in $\mathrm{pH} 3.5$ solution (Figure 5.3b). The data support that, under the testing conditions, $C$. acnes remained alive in the $\mathrm{pH} 3.5$ condition in the absence of glycolic acid. We proceeded to investigate the concentration-dependent killing of $C$. acnes cells in the concentration range of 3.1 to $50 \mathrm{mM}$ glycolic acid. With increasing glycolic acid concentration, the fraction of live cells decreased and complete killing was observed from $25 \mathrm{mM}$ glycolic acid upward, as indicated by the red color stain of all visible cells. This finding is consistent with the MBC data and led to further investigate the effects of glycolic acid treatment on bacterial cell membrane permeability.

\subsubsection{Evaluation of membrane permeabilization}

The effect of glycolic acid treatment on $C$. acnes cell membrane permeability at pH 3.5 was tested by monitoring the release of adenosine triphosphate (ATP), which is a sensitive marker of membrane damage ${ }^{30,31}$. C. acnes cells were incubated with different concentrations of glycolic acid and the amount of ATP released was determined by a bioluminescence readout. Glycolic acid was tested in concentrations in the range of 12.5 to $50 \mathrm{mM}$ along with appropriate controls and the data are presented in Figure 5.4. In untreated C. acnes cells at $\mathrm{pH} 3.5$, the mean ATP concentration was only $93 \mathrm{ng} \mathrm{mL}^{-1}$ while there was a concentration-dependent increase in released ATP with mean values of 298, 451, and $581 \mathrm{ng} \mathrm{mL}^{-1}$ corresponding to treatment with $12.5,25$, and $50 \mathrm{mM}$ glycolic acid, respectively. Noting that the $\mathrm{MBC}$ value was $25 \mathrm{mM}$ in this case, the data indicate that complete killing of $C$. acnes cells occurs when there is a nearly-five-fold increase in membrane permeability. This finding supports that glycolic acid is bactericidal and its mechanism of action involves membrane disruption. Furthermore, Pérez-Isidoro et al. have reported that the nonionic, protonated form of glycolic acid has greater rates of membrane translocation than the ionic, deprotonated form, which helps to explain why glycolic acid exhibits $\mathrm{pH}$-dependent antibacterial activity involving a membrane-disruptive mechanism $^{32}$. 


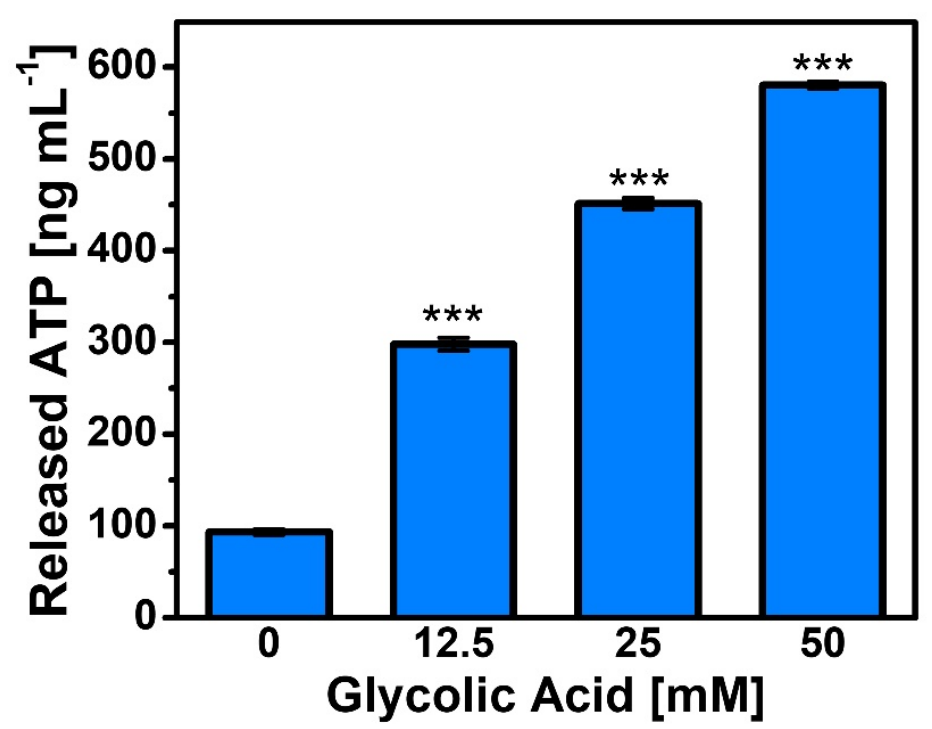

Figure 5.4. Effect of glycolic acid treatment on bacterial cell membrane integrity. The concentration of extracellular ATP released from $C$. acnes cells was measured by bioluminescence assay. The $C$. acnes cell concentration was $2 \times 10^{8} \mathrm{CFU} \mathrm{mL}^{-1}$. Mean \pm standard deviation values are reported from $n=3$ experiments.

\subsubsection{Implications for anti-acne formulation development}

As discussed in the Introduction, glycolic acid is widely used in chemical peels for skincare applications. Notably, the glycolic acid concentrations used in chemical peels are typically high and in the range of $20-50 \mathrm{vol} \%$. While there have been several studies discussing the benefits of glycolic acid chemical peels for overall acne treatment to repair scars and reduce inflammation, there has been only one previous report ${ }^{24}$ that discusses how glycolic acid can directly inhibit $C$. acnes. In that study, a high concentration of $30 \mathrm{vol} \%$ glycolic acid was used and rapid killing was noted in $\mathrm{pH} 1.5$ and 3.5 solutions while less efficient killing was observed at $\mathrm{pH}$ 5.5. Building on this past work, the findings in this study advance mechanistic insight and demonstrate that far lower concentrations of glycolic acid exhibit high bactericidal activity against $C$. acnes. It was discovered that glycolic acid concentrations as low as $0.1,0.2$, and 0.4 vol\% completely kill C. acnes at $\mathrm{pH} 3.0,3.5$, and 4.0, respectively. Importantly, these data reveal that glycolic acid is 150-times more potent at $\mathrm{pH} 3.5$ than previously discussed in the literature and open the door to creating mild anti- 
acne formulations with low-concentration glycolic acid samples for expanded over-thecounter usage.

\subsection{Conclusions}

In this chapter, it was demonstrated that glycolic acid exhibits potent antibacterial activity against $C$. acnes, especially in acidic $\mathrm{pH}$ environments around $\mathrm{pH} 3$ to 4.5 that are suitable for over-the-counter formulation development. While glycolic acid is typically used at relatively high concentrations in chemical peel treatments to exfoliate and unclog pores in deeper level, the findings reveal that glycolic acid is inhibitory at far lower concentrations and devising formulation strategies in this suitable $\mathrm{pH}$ range could lead to more targeted and longer-duration use of glycolic acid to treat acne vulgaris, especially to reduce C. acnes levels while also taking advantage of its broader anti-inflammatory functions. From a mechanistic perspective, there are two key molecular-level insights resulting from this work: (1) the antibacterial potency of glycolic acid against $C$. acnes depends on the solution $\mathrm{pH}$. The data indicate that glycolic acid is more active when it mainly exists in the nonionic (protonated) form, which is consistent with a greater tendency of this form to partition into phospholipid membranes as compared to the anionic (deprotonated) form; and (2) glycolic acid damages the integrity of $C$. acnes cell membranes as part of its bactericidal activity. Considering the longstanding challenges of drug-resistant $C$. acnes strains emerging to conventional antibiotics, the membrane-disruptive mechanism of action of glycolic acid is particularly significant because there is a documented high barrier to bacterial resistance developing against other classes of natural, membrane-active antibacterial drugs such as free fatty acids and similar possibilities might hold for glycolic acid as well. In summary, glycolic acid is a promising antibacterial agent that potently inhibits $C$. acnes in acidic $\mathrm{pH}$ environments suitable for over-the-counter formulations and its membrane-disruptive bactericidal mechanism of action could be useful for improving acne vulgaris treatment strategies. 


\section{References}

1 Williams, H. C., Dellavalle, R. P. \& Garner, S. Acne vulgaris. The Lancet 379, 361372 (2012).

2 Zaenglein, A. L. Acne vulgaris. N. Engl. J. Med. 379, 1343-1352 (2018).

3 Cong, T.-X. et al. From pathogenesis of acne vulgaris to anti-acne agents. Arch. Dermatol. Res. 311, 337-349 (2019).

4 Dessinioti, C. \& Katsambas, A. D. The role of Propionibacterium acnes in acne pathogenesis: facts and controversies. Clin. Dermatol. 28, 2-7 (2010).

5 Kwon, H. H. \& Suh, D. H. Recent progress in the research about Propionibacterium acnes strain diversity and acne: pathogen or bystander? Int. J. Dermatol. 55, 1196-1204 (2016).

6 Dréno, B. et al. Cutibacterium acnes (Propionibacterium acnes) and acne vulgaris: a brief look at the latest updates. J. Eur. Acad. Dermatol. Venereol. 32, 5-14 (2018).

7 Simonart, T. Newer approaches to the treatment of acne vulgaris. Amer. J. Clin. Dermatol. 13, 357-364 (2012).

8 Leccia, M. et al. Topical acne treatments in Europe and the issue of antimicrobial resistance. J. Eur. Acad. Dermatol. Venereol. 29, 1485-1492 (2015).

9 Walsh, T. R., Efthimiou, J. \& Dréno, B. Systematic review of antibiotic resistance in acne: an increasing topical and oral threat. Lancet Infect. Dis. 16, e23-e33 (2016).

10 Dessinioti, C. \& Katsambas, A. Propionibacterium acnes and antimicrobial resistance in acne. Clin. Dermatol.35, 163-167 (2017).

11 Blaskovich, M. A., Elliott, A. G., Kavanagh, A. M., Ramu, S. \& Cooper, M. A. In vitro antimicrobial activity of acne drugs against skin-associated bacteria. Sci. Rep. 9, 1-8 (2019).

12 Nakatsuji, T. et al. Antimicrobial property of lauric acid against Propionibacterium acnes: its therapeutic potential for inflammatory acne vulgaris. J. Invest. Dermatol. 129, 2480-2488 (2009).

13 Yoon, B. K., Jackman, J. A., Valle-González, E. R. \& Cho, N.-J. Antibacterial free fatty acids and monoglycerides: biological activities, experimental testing, and therapeutic applications. Int. J. Mol. Sci. 19, 1114 (2018). 
14 Valle-González, E. R. et al. Characterizing how acidic $\mathrm{pH}$ conditions affect the membrane-disruptive activities of lauric acid and glycerol monolaurate. Langmuir 34, 13745-13753 (2018).

15 Poli, F., Claudel, J.-P., Auffret, N., Leccia, M.-T. \& Dréno, B. Cosmetics and topical medications in acne: Where is the boundary? Ann. Dermatol. Venereol. 144, 768775 (2017).

16 Araviiskaia, E. \& Dréno, B. The role of topical dermocosmetics in acne vulgaris. J. Eur. Acad. Dermatol. Venereol. 30, 926-935 (2016).

17 Tung, R. C., Bergfeld, W. F., Vidimos, A. T. \& Remzi, B. K. $\alpha$-Hydroxy acid-based cosmetic procedures. Amer. J. Clin. Dermatol. 1, 81-88 (2000).

18 Sharad, J. Glycolic acid peel therapy-a current review. Clin., Cosmet. Invest. Dermatol. 6, 281 (2013).

19 Kaminaka, C., Uede, M., Matsunaka, H., Furukawa, F. \& Yamomoto, Y. Clinical evaluation of glycolic acid chemical peeling in patients with acne vulgaris: a randomized, double-blind, placebo-controlled, split-face comparative study. Dermatol. Surg. 40, 314322 (2014).

20 Abels, C. et al. A 10\% glycolic acid containing oil-in-water emulsion improves mild acne: a randomized double-blind placebo-controlled trial. J. Cosmet. Dermatol. 10, 202-209 (2011).

21 Decker, A. \& Graber, E. M. Over-the-counter acne treatments: a review. J. Clin. Aesthet. Dermatol. 5, 32 (2012).

22 Castillo, D. E. \& Keri, J. E. Chemical peels in the treatment of acne: patient selection and perspectives. Clin., Cosmet. Invest. Dermatol. 11, 365 (2018).

23 Wang, X. A theory for the mechanism of action of the $\alpha$-hydroxy acids applied to the skin. Med. Hypotheses 53, 380-382 (1999).

24 Takenaka, Y., Hayashi, N., Takeda, M., Ashikaga, S. \& Kawashima, M. Glycolic acid chemical peeling improves inflammatory acne eruptions through its inhibitory and bactericidal effects on Propionibacterium acnes. J. Dermatol. 39, 350-354 (2012).

25 Bergfeld, W. F. et al. Safety assessment of alpha hydroxy acids as used in cosmetics: Re-review. Int. J. Toxicol. 32, 41S-64S (2013). 
26 Yu, R. J. \& Van Scott, E. J. Alpha-hydroxyacids and carboxylic acids. J. Cosmet. Dermatol. 3, 76-87 (2004).

27 Males, R. \& Herring, F. A 1H-NMR study of the permeation of glycolic acid through phospholipid membranes. Biochim. Biophys. Acta, Biomembr. 1416, 333-338 (1999).

28 Pankey, G. \& Sabath, L. Clinical relevance of bacteriostatic versus bactericidal mechanisms of action in the treatment of Gram-positive bacterial infections. Clin. Infect. Dis. 38, 864-870 (2004).

29 Robertson, J., McGoverin, C., Vanholsbeeck, F. \& Swift, S. Optimisation of the protocol for the LIVE/DEAD BacLight Bacterial Viability Kit for rapid determination of bacterial load. Front. Microbiol. 10, 801 (2019).

30 Oliva, B., O’Neill, A. J., Miller, K., Stubbings, W. \& Chopra, I. Antistaphylococcal activity and mode of action of clofazimine. J. Antimicrob. Chemother. 53, 435-440 (2004).

31 O'Neill, A. J., Miller, K., Oliva, B. \& Chopra, I. Comparison of assays for detection of agents causing membrane damage in Staphylococcus aureus. J. Antimicrob. Chemother. 54, 1127-1129 (2004).

32 Pérez-Isidoro, R., Sierra-Valdez, F. \& Ruiz-Suárez, J. Anesthetic diffusion through lipid membranes depends on the protonation rate. Sci. Rep. 4, 7534 (2014).

33 Lood, R. \& Collin, M. Characterization and genome sequencing of two Propionibacterium acnes phages displaying pseudolysogeny. BMC Genomics 12, 198 (2011). 


\section{Chapter 6}

\section{Engineering Phospholipid-Glycerol Monolaurate Bicellar Systems with Membrane-Disruptive Activity against Synthetic and Bacterial Cell Membranes*}

Composed of long- and short-chain phospholipids, bicelles are interesting membrane mimics classically used as carriers for other molecules in structural biology and drug delivery. Recently, they have been employed for the fabrication of supported lipid bilayers (SLBS) utilizing new bicelle compositions. The synthetic short-chain phospholipids are replaced with naturally abundant lipids that have useful properties. In this chapter the use of glycerol monolaurate (GML) was studied, as a short-chain phospholipid replacement for SLB fabrication and antibacterial applications. Using quartz crystal microbalance-dissipation and fluorescence microscopy the formation of SLB from phospholipid-GML bicelles up to the q-ratio (ratio of longchain phospholipid to GML) of 2.5 was investigated. The results suggested that $q=0.25$ is suitable for antibacterial applications. So using the colony-forming unit enumeration assay the bactericidal effect of $q=0.25$ bicelles was evaluated on Staphylococcus aureus bacteria. Altogether, the findings highlight the feasibility of engineering phospholipid-monoglyceride bicelles for SLB fabrication and other relevant applications such as in skincare formulations.

\footnotetext{
*This chapter was partially published as Sut, T. N; Valle-González, E. R.; Yoon, Bo Kyeong; Park, Soohyun; Jackman, Joshua A.; Cho, Nam-Joon. Bicelle Engineering for Membrane-Disruptive Antibacterial Applications. Applied Materials Today (2021).
} 


\subsection{Introduction}

Bicelles (also known as bicellar systems, bilayered mixed micelles or lipid nanodiscs) are cell membrane mimics made from long- and short-chain phospholipids ${ }^{1-5}$, which form $2 \mathrm{D}$ disk-shaped structures in general when mixed, as a result of the long-chain phospholipids self-assembling into a bilayer plane and the short-chain phospholipids rim around the bilayer edges. Indeed, bicelles can have a broad array of morphologies (e.g., worm-shaped micelles, lamellar sheets with holes, vesicles-shaped aggregates) depending on the environmental factors such as total lipid concentration, q-ratio (long- to short-chain phospholipid molar ratio), lipid composition and temperature 6-12. The traditional applications of bicelles have been in the structural biology field ${ }^{13-16}$, where they provide membranous environment for encapsulating membrane-associated molecules (e.g., membrane proteins) to help study the structural and functional properties of those molecules. There have also been emerging applications in drug delivery ${ }^{17-20}$, where bicelles act as structurally delivery vehicles for hydrophobic drugs and have been known to enhance drug solubility and cellular uptake. Particularly, bicelles are beneficial for transdermal drug delivery applications due to their structure and composition which permit the passage through stratum corneum of the skin without any damage ${ }^{21-29}$.

In addition to serving as material platforms that support the structure and function of other molecules (i.e., being membrane hosts or carriers), bicelles have been recently used as stand-alone functional entities in the biointerfacial science field where they exhibit their own active functions. A prominent example is the fabrication of supported lipid bilayers (SLBs) ${ }^{30-32}$. Formed on hydrophilic solid surfaces, SLBs are compatible with surfacesensitive measuring techniques ${ }^{33,34}$ and therefore useful in various coating and sensing applications ${ }^{35-43}$. Zeineldin et al first demonstrated the bicelle-based SLB fabrication on silicon chips 44 and many others have followed suit to gain a deeper mechanistic understanding in various aspects ${ }^{45-52}$. These studies have proven that bicelles can actively interact with and reassemble on inorganic surfaces to form SLB, and in fact, offer superior advantages to vesicles ${ }^{53}$ - the most widely used SLB fabrication tools - in some respects (e.g., easier sample preparation) ${ }^{54}$. 
Most recently, it has been reported that bicelles made of long-chain phospholipids and naturally abundant lipids such as lauric acid ${ }^{55}$ and monocaprin ${ }^{56}$, each of which is a type of fatty acids and monoglycerides, respectively, can form SLB on silica surfaces. The main motivation behind these studies was to explore more bicelle options for SLB fabrication by replacing the synthetic short-chain phospholipids with the natural as well as abundant lipid sources. The results pointed to the feasibility of finetuning key bicelle components for SLB fabrication in the context of bicelles being actively functional at solid-liquid interfaces as opposed to being passive hosts in solution-phase applications. Note that, when used as hosts, the short-chain phospholipids or zwitterionic surfactants such as 3-([3cholamidopropyl] dimethylammonio)-2-hydroxy-1-propanesulfonate in bicelles are meant to preserve and/or enhance the properties of the incorporated molecules ${ }^{57-59}$. By contrast, when used as SLB tools, the natural lipids in bicelles are actively involved in bicellesubstrate interactions during SLB formation. Interestingly, they also possess membraneactive properties, and this spurred the further exploration of application possibilities as pertinent to such properties and ask whether it could be achievable to engineer their membrane-active properties in bicelle form as functional structures for relevant applications besides SLB fabrication.

To this end, one promising option to incorporate as a functional component within bicelles is a monoglyceride derivative of lauric acid called glycerol monolaurate (GML). It is a non-ionic surfactant that can inhibit bacteria and viruses via membrane disruption when in aggregated form ${ }^{60-64}$. It has also been reported to be a promising next-generation antibacterial solution when formulated as nano-emulsions ${ }^{65}$. However, the use of GML in bicelle formulation has yet to be explored and so has its potential application as an SLB tool. In addition, given the potent membrane-disruptive activities of GML, it would be interesting to explore if bicelles formed with GML as one of the main components can be engineered to still exhibit such activities and thus are suitable for relevant applications such as skincare formulations.

Hence, in this study, GML was employed in making bicelles and characterized the SLB formation capability as well as the membrane-disruptive activity of GML-containing 
bicelles for potential skincare applications (Figure 6.1.). The long-chain phospholipid was 1,2-dioleoyl-sn-glycero-3-phosphocholine (DOPC) and the freeze-thaw-vortex cycling was used to prepare DOPC/GML bicelles. For SLB formation evaluation, the quartz crystal microbalance-dissipation (QCM-D) and fluorescence microscopy experiments were performed to measure the adsorption kinetics of bicelles, observe the morphology of the adsorbed layers and determine the membrane fluidity, respectively. Furthermore, for biological membrane-disruptive activity evaluation, minimum bactericidal concentration (MBC) of DOPC/GML bicelles was tested against Staphylococcus aureus (S. aureus) bacteria along with visual observation of the bicelle formulation using the cryogenic transmission electron microscopy (cryo-TEM), and additional support for the biological activity of the bicelle was confirmed with Live/Dead assay. The safeness of the bicelles or bicellar system on skin cells was verified with a cell cytotoxicity assay to validate its possible usage on skincare formulations.

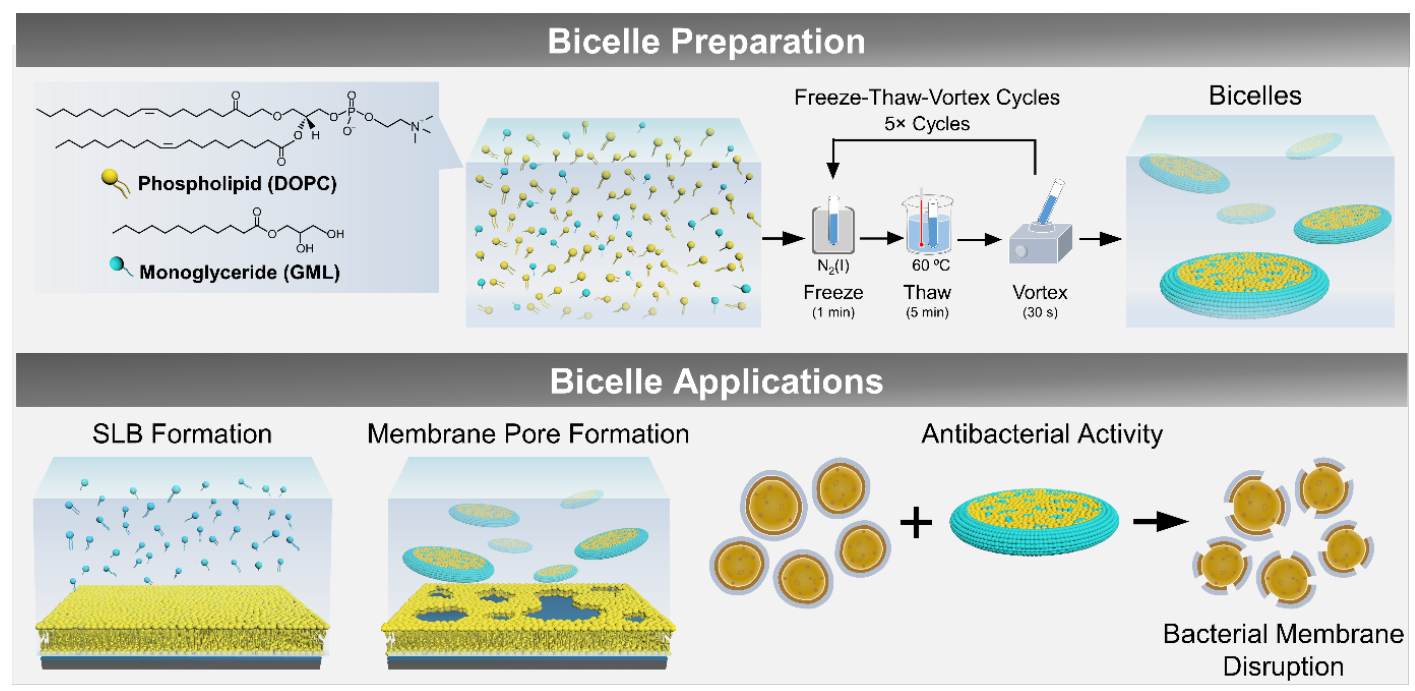

Figure 6.1. Overall experimental strategy. Bicelles were prepared by hydrating the lipid mixtures of DOPC and GML, and then processing the mixtures with the freeze-thaw-vortex cycling method. The resulting bicelles were evaluated for SLB formation and antibacterial activity. 


\subsection{Materials and Methods}

\subsubsection{Reagents}

1,2-dioleoyl-sn-glycero-3-phosphocholine （DOPC), 1,2-dihexanoyl-sn-glycero-3phosphocholine (DHPC), and 1,2-dioleoyl-sn-glycero-3-phosphoethanolamine-N(lissamine rhodamine B sulfonyl) (ammonium salt) (Rh-PE) lipids dispersed in chloroform were purchased from Avanti Polar Lipids (Alabaster, AL, USA). Glycerol monolaurate (GML) was obtained from Abcam (Cambridge, UK). Luria-Bertani (LB) broth and Mueller-Hinton (MH) agar were obtained from BD (Sparks, MD) and Sigma-Aldrich (St Louis, MO), respectively. The Live/Dead BacLight Bacterial Viability Kit was purchased from Invitrogen/Molecular Probes (Carlsbad, CA). Minimum Essential Medium (MEM) Alpha Modification and fetal bovine serum (FBS) were bought from Hyclone (Logan, UT). Phosphate-buffered saline (PBS), Penicillin-Streptomycin $(10,000 \mathrm{U} / \mathrm{mL})$ and $0.25 \%$ Trypsin-EDTA solution were procured from Gibco (Carlsbad, CA). The cell counting kit8 (CCK-8 ) was from Dojindo Molecular Technologies (Rockville, MD). Milli-Q-treated water (>18 M $\Omega \cdot \mathrm{cm}$ resistivity) from Millipore Sigma (Burlington, MA, USA) was used to prepare all solutions. For SLB experiments, bicelle samples were prepared in buffer that contained $10 \mathrm{mM}$ Tris and $150 \mathrm{mM} \mathrm{NaCl}(\mathrm{pH}$ 7.5) and for bacterial experiments, in PBS.

\subsubsection{Bicelle Preparation}

Lipid hydration and subsequent freeze-thaw-vortex cycling were performed to prepare bicelles, as previously described ${ }^{49}$. The DOPC lipids (or DOPC with $0.5 \mathrm{~mol} \% \mathrm{Rh}-\mathrm{PE}$ lipids) were placed in a glass vial and dried with nitrogen gas to form a lipid film on the wall, which was then kept in a vacuum desiccator overnight to completely remove chloroform residues. Next, the DOPC film was hydrated to $1 \mathrm{mM}$ in Tris buffer containing 20, 4 and $0.4 \mathrm{mM}$ GML to prepare mixtures with q-ratios of $0.05,0.25$ and 2.5. The resulting lipid suspensions were then subjected to five freeze-thaw-vortex cycles which involved the following steps: 1-min freezing in liquid nitrogen, 5 -min thawing in a $60{ }^{\circ} \mathrm{C}$ water bath and 30-s vortexing. Immediately before the experiment, an aliquot of the stock 
lipid suspension was diluted in the buffer to the desired final lipid concentration. For fabrication of SLBs in bicelle-SLB interaction experiments, DOPC/DHPC bicelles at $\mathrm{q}=$ 0.25 were prepared and for bacterial experiments, DOPC/GML bicelles at $\mathrm{q}=0.25$ were prepared in PBS.

\subsubsection{Quartz Crystal Microbalance-Dissipation (QCM-D)}

Bicelle adsorption experiments were conducted using a Q-Sense E4 instrument (Biolin Scientific AB, Stockholm, Sweden). Before each experiment, the silicon oxide-coated quartz crystal sensors were cleaned with the following steps: rinsing with water and ethanol, drying with nitrogen gas and treating for $1 \mathrm{~min}$ in an oxygen plasma chamber (PDC-002, Harrick Plasma, Ithaca, NY). The temperature of the QCM-D chambers was maintained at $25^{\circ} \mathrm{C}$. All solutions were flowed continuously using a peristaltic pump (Reglo Digital MS-4/6, Ismatec, Wertheim, Germany) at a flow rate of $50 \mu \mathrm{L} / \mathrm{min}$. Measurement data were collected at multiple odd overtones by the Q-Soft software package (BiolinScientific $\mathrm{AB}$ ). The reported data were collected at the $5^{\text {th }}$ overtone and normalized according to the overtone number. Data processing was completed using the Q-Tools (Biolin Scientific AB) and OriginPro (OriginLab, Northampton, MA) software programs.

\subsubsection{Epifluorescence Microscopy}

Imaging experiments were conducted using a Nikon Eclipse Ti-E inverted microscope with a $60 \times$ oil-immersion objective (NA 1.49). The excitation source was a mercury-fiber illuminator C-HGFIE Intensilight (Nikon, Tokyo, Japan), and the light was passed through a TRITC filter block. An Andor iXon3 897 EMCCD camera was used to obtain the images at the rate of 1 frame per $3 \mathrm{~s}$. The samples were injected into a microfluidic chamber (sticky-Slide VI 0.4, ibidi GmbH, Martinsried, Germany) at a flow rate of $50 \mu \mathrm{L} / \mathrm{min}$ using a peristaltic pump (Reglo Digital MS-4/6). All measurements were performed at $\sim 25^{\circ} \mathrm{C}$. 


\subsubsection{Bacterial Cell Culture}

Staphylococcus aureus (S. aureus), ATCC 25923, (American Type Culture Collection, Manassas, VA) was cultured in LB broth overnight at $37^{\circ} \mathrm{C}$. The overnight suspension was inoculated in fresh LB broth and cultured under aerobic conditions until reaching an $\mathrm{OD}_{600}$ of 0.5 (exponential growth phase). The bacterial cells were next harvested by centrifugation at $1485 \times g$ for $10 \mathrm{~min}$, washed thrice with PBS, and re-suspended in the same buffer. The $\mathrm{OD}_{600}$ value was measured and the appropriate dilution was made to reach a value of $\sim 0.1$, which corresponds to a density around $4 \times 10^{7} \mathrm{CFU} \mathrm{mL}^{-1}$, as confirmed by colony-forming unit (CFU) enumeration. Afterwards, appropriate dilutions were made with PBS buffer to obtain the required bacterial cell density for each experiment.

\subsubsection{Cryogenic Transmission Electron Microscopy (Cryo-TEM)}

The morphological structure of the DOPC/GML bicelle at q-ratio of 0.25 was described by cryo-TEM. The sample was vitrified using a Vitrobot Mark IV (Thermo Fisher, Waltham, MA). Initially, a $4 \mu \mathrm{l}$ volume at the highest concentration of the bicellar was placed onto a cooper holy carbon grid (Quantioil R2/2, Jena, DE). The cryogrid was blotted with filter paper to enable the formation of a thin film of the sample and full coverage of the grid holes. The blotting time was 2 seconds without force. The film was frozen in liquid ethane $\left(-196{ }^{\circ} \mathrm{C}\right)$ and kept in liquid nitrogen until examination. The visualization of the sample was done with a microscope Tecnai Arctica (Thermo Fisher, Waltham, MA) at $200 \mathrm{kV}$ using low-dose imaging conditions.

\subsubsection{Minimum Bactericidal Concentration (MBC) Testing}

For bactericidal activity of testing samples, GML concentrations in DOPC/GML bicelles at $\mathrm{q}=0.25$ from 0.031 to $2 \mathrm{mM}$ (2-fold serial dilutions) were treated against $S$. aureus $(5 \mathrm{x}$ $10^{5} \mathrm{CFU} \mathrm{mL} \mathrm{m}^{-1}$ ) and it incubated for $3 \mathrm{~h}$ at $37^{\circ} \mathrm{C}$. Afterwards, the samples were diluted in a 10 -fold series, streaked onto $\mathrm{MH}$ agar plates and incubated overnight at $37^{\circ} \mathrm{C}$. The $\mathrm{MBC}$ 
value was defined as the minimum concentration of testing sample at which it removes $99.99 \%$ of the bacterial cell count. All experiments were performed in triplicate.

\subsubsection{Live/Dead Bacterial Cell Staining}

For visual observation of antibacterial activity of the bicelle samples, GML concentrations in DOPC/GML bicelles at $\mathrm{q}=0.25$ from 0.031 to $1 \mathrm{mM}$ (2-fold serial dilutions) were added to $S$. aureus suspensions at a final concentration of $1 \times 10^{7} \mathrm{CFU} \mathrm{mL}^{-1}$. The samples were incubated for $3 \mathrm{~h}$ at $37^{\circ} \mathrm{C}$ and then stained with the dyes from the Live/Dead BacLight Bacterial Viability Kit (Molecular Probes, Invitrogen, Carlsbad, CA). Positive and negative (treated with 70\% (v/v) ethanol) controls were also prepared following the manufacture's protocol. Stained bacteria were observed with LSM 710 confocal laser scanning microscope (Zeiss, Oberkochen, Germany). Live bacteria are dyed in green while dead in red.

\subsubsection{Cell Viability Testing}

Human immortalized keratinocytes cells (HaCaT) (CLS Cell Lines Service, Eppelheim, Germany) were maintained in Minimum Essential Medium (MEM) supplemented with $10 \%$ fetal bovine serum, $100 \mathrm{U} \mathrm{mL}^{-1}$ penicillin and $100 \mathrm{mg} \mathrm{mL}^{-1}$ streptomycin in a humidified atmosphere with $5 \% \mathrm{CO}_{2}$ at $37^{\circ} \mathrm{C}$. Cells were subculture from the culture flask using $0.25 \%$ Trypsin-EDTA solution. Cell proliferation and viability were quantified using a cell counting kit-8 assay (CCK-8) (Dojindo Molecular Technologies, Rockville, MD) according to the manufacturer's protocol. A HaCaT density of 5000 cells per well were seeded in a 96 well plate and allowed to grow overnight. The next days, cell were treated with different concentration of the DOPC/GML (q-ratio 0.25) bicelles - $0.5 \mathrm{mM}$ to $2 \mathrm{mM}$ - and further incubated for $72 \mathrm{hrs}$. Cells treated with Triton X-100 were used as positive control. CCK reagent was diluted 10 times with culture media and incubated with cells for $1 \mathrm{~h}$ at $37^{\circ} \mathrm{C}$, the colorimetric culture media absorbance were measured with a plate reader (Infinite 200 PRO microplate reader, Tecan, Zurich, Switzerland).) at $450 \mathrm{~nm}$ wavelength. Experiments were conducted in triplicate. 


\subsubsection{Statistical analysis}

The statistical analysis was performed using the OriginPro software. Unpaired Student's ttest and one-way ANOVA with Tukey's multiple comparison were used to calculate the statistical significance of data sets. A $P$ value of less than 0.05 was considered statistically significant. Data are presented as the mean \pm standard deviation.

\subsection{Results and Discussion}

\subsubsection{Evaluation of Bicelle Adsorption and SLB Formation}

\subsubsection{QCM-D}

To monitor the adsorption kinetics of DOPC/GML bicelles, the QCM-D experiments were performed using silicon dioxide surfaces and selected test conditions (q-ratios of 0.05, 0.25 and 2.5). The QCM-D technique measures the shifts in frequency $(\Delta \mathrm{f})$ and energy dissipation $(\Delta \mathrm{D})$ of the piezoelectric quartz sensors that reflect the changes in mass and viscoelastic properties, respectively, of the adsorbed layers ${ }^{66}$. The kinetic data are obtained from the $\Delta \mathrm{f}$ and $\Delta \mathrm{D}$ shifts which are recorded as a function of time during the entire adsorption process. In the experiments, a stable baseline signal was first established in the measurement chambers with the buffer. Bicelles were then added under continuous flow conditions and after the lipid adlayers were formed, a buffer washing step was done over 10 min. After complete washing step, whether SLB formation occurred or not was evaluated by reading the final $\Delta \mathrm{f}$ and $\Delta \mathrm{D}$ shifts. The typical final $\Delta \mathrm{f}$ and $\Delta \mathrm{D}$ values reported in the literature for high-quality phospholipid SLBs are around $-25 \mathrm{~Hz}$ and $<1 \times 10^{-6}$, respectively ${ }^{66}$. Since DOPC lipids are the main SLB component, the DOPC concentration is marked alone in Figure 6.2. to present the data (the corresponding GML concentration can be computed from the q-ratio). 
$q=0.05$

Figure 6.2A presents the adsorption kinetics for bicelle adsorption with different concentrations at $\mathrm{q}=0.05$ where the bicelles had 20-fold greater amount of GML than DOPC. At 0.5-0.031 mM DOPC, a two-step kinetics indicating bicelles adsorb until they reach the critical surface coverage first and then fuse/rupture ${ }^{49}$ to form SLB was observed. At $0.016 \mathrm{mM}$ DOPC, the degree of bicelle rupture was insignificant, as indicated by a small increase in $\Delta \mathrm{f}$ and decrease in $\Delta \mathrm{D}$ from the critical coverage point to the final values, and hence no SLB formed.

The corresponding final $\Delta \mathrm{f}$ and $\Delta \mathrm{D}$ shifts are presented in Figure 6.2B. At 0.5 mM DOPC, the final $\Delta \mathrm{f}$ and $\Delta \mathrm{D}$ values were around $-26.5 \pm 2.8 \mathrm{~Hz}$ and $3.8 \pm 1.0 \times 10^{-6}$, respectively. Similar results were obtained at $0.25-0.031 \mathrm{mM}$ DOPC with the final $\Delta \mathrm{f}$ shifts ranging around -23 to $-25 \mathrm{~Hz}$ and the $\Delta \mathrm{D}$ shifts around 1.8 to $3.1 \times 10^{-6}$. The final $\Delta \mathrm{f}$ shifts are consistent with SLB formation, however the final $\triangle \mathrm{D}$ shifts are higher than the SLB values, suggesting the formed SLBs consist of some unruptured bicelles ${ }^{55,56}$. For the $0.016 \mathrm{mM}$ DOPC case, the final values were around $-50.6 \pm 2.8 \mathrm{~Hz}$ and $16.9 \pm 0.8 \times 10^{-6}$, respectively, and thus it is likely that the formed layer from bicellar adsorbates on solid surface was mostly made up of unruptured bicelles. Altogether, the results suggest that SLBs can be formed with DOPC/GML bicelles at $\geq 0.031 \mathrm{mM} \mathrm{DOPC}$ at $\mathrm{q}=0.05$.

$q=0.25$

Figure 6.2C presents the adsorption kinetics for bicelle adsorption with different concentrations at $\mathrm{q}=0.25$ where the bicelles had 4-fold greater amount of GML than DOPC. In all cases, the kinetics showed the establishment of the critical surface coverage and subsequent rupture. However, the kinetic profiles were complicated and this could be likely attributed by GML which is a non-ionic surfactant and has been reported to exert membrane-destructive activities even at concentrations lower than its critical micelle concentration $(\mathrm{CMC})^{64}$ of around $60 \mu \mathrm{M} .{ }^{67}$ Note that for $0.5-0.031 \mathrm{mM}$ DOPC, the GML concentration is $2-0.124 \mathrm{mM}$ which is above CMC and for $0.016 \mathrm{mM}$ DOPC, $0.064 \mathrm{mM}$ which is near CMC. The GML activity was indicated by the decreasing $\Delta \mathrm{f}$ and increasing $\triangle \mathrm{D}$ shifts after bicelle rupture which would otherwise stabilize if SLB formation was complete without any disruption. The GML-induced effects were less pronounced at 0.063 - 
$0.016 \mathrm{mM}$ DOPC, likely due to lower concentrations. Interestingly, the effects of GML were not observed at $\mathrm{q}=0.05$ for all test concentrations although the GML concentration was 5 times greater in each case. This could be the result of membrane softening as well as possible membrane translocation by GML that is already at play in the bulk solution due to high GML concentration and in turn supports the bicelle deformation on the substrate without causing further destabilization during bicelle rupture.

Figure 6.2D presents the final $\Delta \mathrm{f}$ and $\Delta \mathrm{D}$ shifts of the adsorbed layers. At $0.5 \mathrm{mM}$ DOPC, the final $\Delta$ f and $\Delta \mathrm{D}$ values reached around $-27.4 \pm 2.4 \mathrm{~Hz}$ and $8.4 \pm 1.1 \times 10^{-6}$, respectively. Since the $\Delta \mathrm{D}$ is significantly higher than the SLB value, the adsorbed layer cannot be an $\mathrm{SLB}$. At $0.25 \mathrm{mM}$ DOPC and below, the final $\Delta \mathrm{f}$ and $\Delta \mathrm{D}$ values ranged approximately -23 to $-25 \mathrm{~Hz}$ and 1.5 to $4.5 \times 10^{-6}$, respectively. Again, although the $\Delta \mathrm{f}$ values fall within an SLB range, the high $\triangle \mathrm{D}$ values indicate the likely presence of some unruptured bicelles in the SLBs ${ }^{55,56}$. It is also possible that these are not SLBs and the $\triangle \mathrm{D}$ values are lower than those at $0.5 \mathrm{mM}$ DOPC (a non-SLB condition) only because the total lipid concentrations are lower. Taken together, the results at $\mathrm{q}=0.25$ show that SLB formation with DOPC/GML bicelles is deterred by the effects of GML and therefore not recommended.

$q=2.5$

Figure 6.2E presents the adsorption kinetics for bicelle adsorption with different concentrations at $\mathrm{q}=2.5$ where the bicelles had 2.5-fold greater amount of DOPC than GML. In all cases, SLB formation occurred via two-step kinetics, and as presented in Figure 6.2F, the final $\Delta \mathrm{f}$ values ranged around -25 to $-29 \mathrm{~Hz}$ at $0.5-0.016 \mathrm{mM}$ DOPC whereas the final $\Delta \mathrm{D}$ values reached around 0.5 to $1.5 \times 10^{-6}$ at $0.5-0.031 \mathrm{mM} \mathrm{DOPC}$ and around $3.5 \times 10^{-6}$ at $0.016 \mathrm{mM}$ DOPC. The SLB formation was very slow and took more than $2 \mathrm{~h}$ and $4 \mathrm{~h}$ at 0.031 and $0.016 \mathrm{mM}$ DOPC, respectively. Therefore, the best SLB conditions for DOPC/GML bicelles at $\mathrm{q}=2.5$ are with $0.5-0.063 \mathrm{mM}$ DOPC.

In summary, the QCM-D results demonstrate that DOPC/GML bicelles can form SLBs at $\mathrm{q}=0.05$ with $\geq 0.031 \mathrm{mM}$ DOPC and at $\mathrm{q}=2.5$ with $\geq 0.063 \mathrm{mM}$ DOPC, whereas direct SLB formation is unfavorable at $\mathrm{q}=0.25$. 

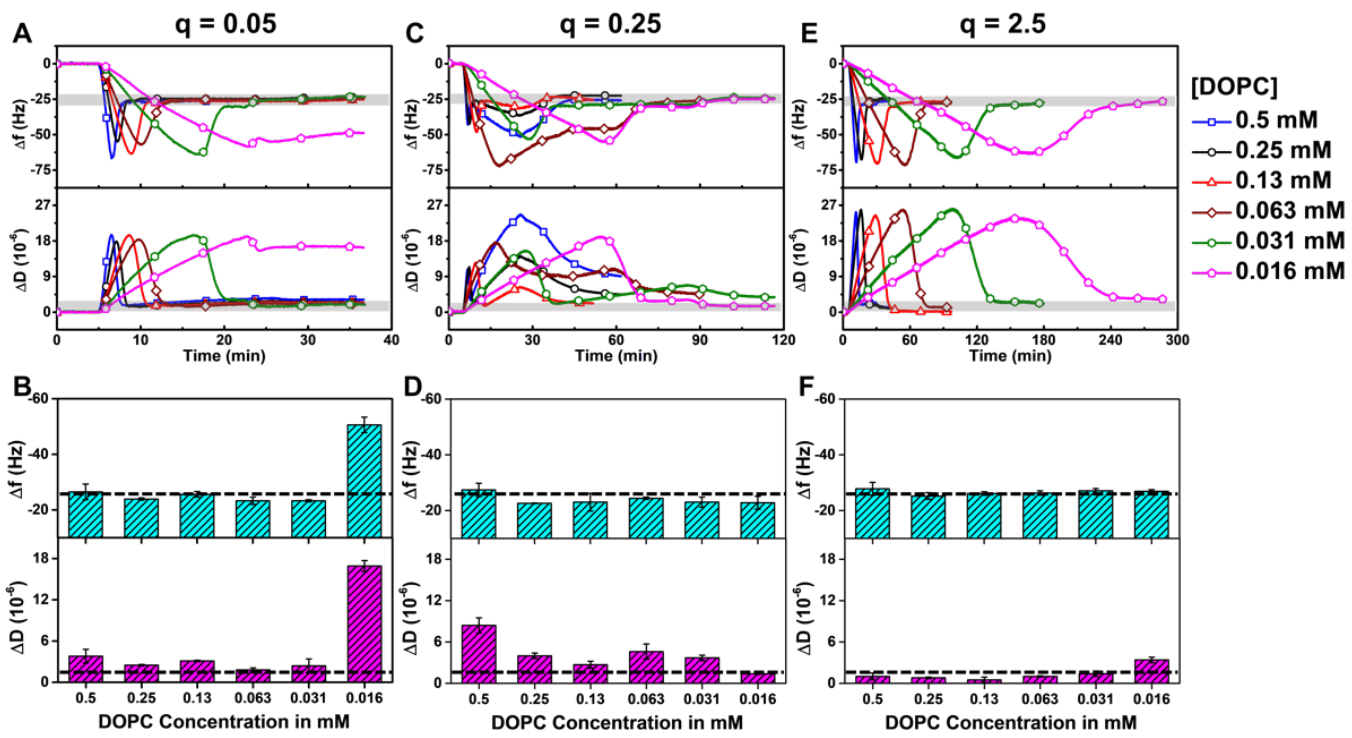

Figure 6.2. QCM-D results of DOPC/GML bicelle adsorption onto silicon dioxide at different q-ratios. (A) Frequency and dissipation shifts, $\Delta \mathrm{f}$ (upper panel) and $\Delta \mathrm{D}$ (lower panel), respectively, were recorded as a function of time at $\mathrm{q}=0.05$. The shaded areas represent the range of shifts an SLB. (B) Column graph of final shifts (upper panel for $\Delta \mathrm{f}$ and lower panel for $\Delta \mathrm{D}$ ) $v s$ lipid concentration for bicelles at $\mathrm{q}=0.05$. The dotted lines represent the typical values for an SLB. Corresponding results for bicelle adsorption at (C-D) $q=0.25$ and $(\mathbf{E}-\mathbf{F}) \mathrm{q}=2.5$.

\subsubsection{2. $\quad$ Epifluorescence Microscopy}

The epifluorescence microscopy experiments were performed to visually observe the process of bicelle adsorption and membrane formation on a glass surface. For imaging purposes, bicelles were labelled with $0.5 \mathrm{~mol} \%$ of Rh-PE fluorescent lipids (with respect to DOPC $\mathrm{mol} \%$ ). The following representative conditions were chosen for each q-ratio based on the QCM-D results: $\mathrm{q}=0.05$ at 0.031 and $0.016 \mathrm{mM}$ DOPC for SLB and nonSLB cases, respectively; $\mathrm{q}=0.25$ at 0.5 and $0.063 \mathrm{mM}$ DOPC for high and low GML activity cases, respectively; and $\mathrm{q}=2.5$ at $0.063 \mathrm{mM}$ DOPC for SLB cases. Time-lapsed images were captured as the bicelles were injected into a microfluidic chamber under continuous flow. The time the bicelles reached the chamber was defined as $t=0 \mathrm{~min}$ and the micrographs at different time points after $t=0 \mathrm{~min}$ were recorded for the entire adsorption process. The images are presented in Figure $\mathbf{6 . 3}$ and the results are discussed below. 
$q=0.05$

At $0.031 \mathrm{mM}$ DOPC, the fluorescence intensity increased as the bicelles adsorbed increasingly and reached the critical surface after around $9.5 \mathrm{~min}$ (Figure 6.3A). Some dark patches also appeared and their boundaries kept expanding, which suggests bicelle fusion and SLB propagation. After the patches stopped growing at around $19.5 \mathrm{~min}$, the buffer washing step was performed. The final layer had two phases - a brighter region indicative of comprising aggregates of unfused bicelles and a darker region of fused bicelles where the unlabeled GML molecules were likely distributed throughout the region during bicelle fusion. This phase separation is reminiscent of how GML affects the lipid phase dynamics in the membrane ${ }^{68}$ as well as the clustering arrangements of molecules at the membrane ${ }^{69}$. It also supports the QCM-D data that showed SLB formation at $\mathrm{q}=0.05$ with $\geq 0.031 \mathrm{mM}$ DOPC and confirms the high $\Delta \mathrm{D}$ values are related to formation of a phase-separated SLB containing both regions of unfused and fused bicelles.

At $0.016 \mathrm{mM}$ DOPC, the critical surface coverage was reached after around 9.4 min with two separate phases - a brighter one suggestive of containing aggregates of intact bicelles and a darker one of ruptured bicelles (Figure 6.3B). There was rarely any propagation afterwards, which is consistent with the minimal changes in post-critical surface coverage QCM-D signals, and the buffer wash was performed after the fluorescence signals stabilized at around $15.8 \mathrm{~min}$. The appearance of black spots on the bright phase postwashing could be due to accumulation/redistribution of GML molecules within the adsorbed layer rather than continuation of bicelle fusion and bilayer propagation, as can be inferred from the high final QCM-D shifts.

$q=0.25$

Bicelle adsorption at $0.5 \mathrm{mM}$ DOPC proceeded quickly and the fluorescence intensity kept increasing until after around $20 \mathrm{~min}$ (Figure 6.3C). Upon the buffer wash, a small number of dark spots appeared. There was no apparent bicelle fusion or subsequent bilayer propagation and based on the final QCM-D shifts at this concentration, the adlayer can be considered as a non-SLB. 
At $0.063 \mathrm{mM}$ DOPC, bicelle adsorption was slow and the fluorescence signals stabilized at around $40 \mathrm{~min}$ (Figure 6.3D). The buffer wash was then performed, and the final layer contained several dark spots suggestive of GML molecules and/or defects. Bicelle fusion was not observed either.

Overall, the fluorescence microscopy images lacked the visual characteristics of bicelle fusion and bilayer propagation processes, and hence further confirm that $q=0.25$ is not suitable for SLB formation.

$q=2.5$

Bicelles adsorbed gradually at $0.063 \mathrm{mM}$ DOPC and reached the critical surface coverage after around $16 \mathrm{~min}$, followed by bicelle fusion and bilayer propagation (Figure 6.3E). The SLB formation was completed after around $20 \mathrm{~min}$, and upon washing, a small number of bright spots indicative of unruptured bicelles remained in the SLB. This result agrees well with the QCM-D data which showed SLB formation. 


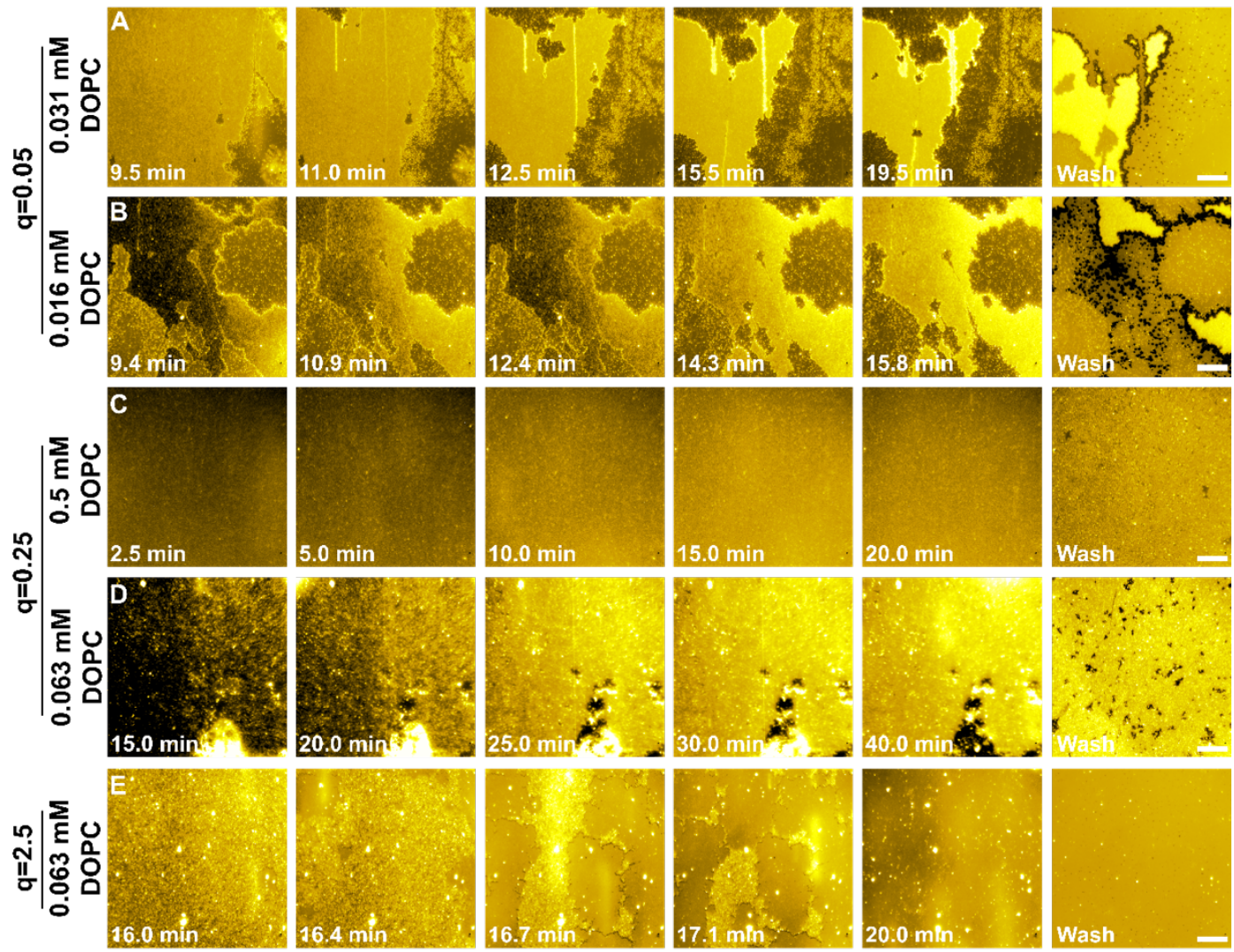

Figure 6.3. Fluorescence microscopy imaging of the representative DOPC/GML bicelle adsorption cases at different q-ratios. Bicelles were added onto the glass surface at $t=0 \mathrm{~min}$ and the fluorescence microscopy images were recorded for the entire adsorption process. Bicelle test conditions are $\mathrm{q}=0.05$ at (A) $0.031 \mathrm{mM}$ DOPC and (B) $0.016 \mathrm{mM} \mathrm{DOPC}, \mathrm{q}=0.25$ at (C) $0.5 \mathrm{mM}$ DOPC and (D) $0.063 \mathrm{mM}$ DOPC, and $\mathrm{q}=2.5$ at (E) $0.063 \mathrm{mM}$ DOPC. Scale bars are $20 \mu \mathrm{m}$.

\subsubsection{Evaluation of Membrane-Disruptive Activity of Bicelles}

Another potential benefit of incorporating GML as a functional element within bicelles is antibacterial activity related to its membrane-disruptive activity. The use of bicelles in antibacterial/antimicrobial applications has been as model membranes to study how the antibacterial/antimicrobial agents interact with and/or affect them ${ }^{70-75}$, but not as main functional agents to investigate their membrane activities/effects. Therefore, herein, it was evaluated the potential of DOPC/GML bicelles to cause membrane disruption by depositing them onto SLBs as well as bacterial cell membranes. The choice of bicelle conditions was based on GML concentration only since GML is the main component for 
membrane-disruptive activity ( $v s$ only DOPC concentration was presented in the SLB data as DOPC is the main component for SLB formation). Thus, $0.125 \mathrm{mM} \mathrm{GML} \mathrm{was} \mathrm{selected,}$ which is about twice the CMC $\left(\sim 60 \mu \mathrm{M}^{67}\right)$, because GML has been shown to have pronounced membrane activity at this concentration ${ }^{64}$. First, the prefabricated SLB platform was employed to observe membrane destabilization responses including kinetics of bicelle-membrane interactions in an engineered model system, and then the membranedisruptive activities of the bicelles were further confirmed in a biological system using $S$. aureus.

\subsubsection{Interaction of DOPC/GML Bicelles with DOPC SLBs}

To study the bicelle-membrane interaction on SLBs, the QCM-D experiments were conducted with the prefabricated SLBs. First, DOPC/DHPC bicelles at $\mathrm{q}=0.25$ and 0.031 mM DOPC were adsorbed on silicon oxide surfaces to form SLBs, as previously described 49. These SLBs were composed of DOPC lipids only and had high quality, i.e., complete and free of defects, as previously proven ${ }^{49}$. Then, DOPC/GML bicelles were added onto the formed SLBs and the kinetics of bicelle-SLB interactions were observed by changes in QCM-D profiles. All three q-ratios of $0.05,0.25$ and 2.5 were tested, and the results are presented in Figure 6.4.

Figure 6.4A shows the kinetics of the bicelle-SLB interactions at all three q-ratios. The SLB shifts were normalized to zero baseline signals for direct comparison of the kinetics and magnitudes of interactions between q-ratios, as caused by bicelle addition. Figure 6.4B complements this comparison by showing in column graphs the peak $\Delta \mathrm{f}$ and $\Delta \mathrm{D}$ shifts during bicelle-SLB interactions. At $\mathrm{q}=0.05$, there was a decrease in $\Delta \mathrm{f}$ to around $-20.1 \pm$ $0.8 \mathrm{~Hz}$ and a concomitant increase in $\Delta \mathrm{D}$ to around $6.3 \pm 0.2 \times 10^{-6}$, which is indicative of bicelle membrane intercalation. At $q=0.25$, similar kinetics were observed with smaller $\Delta \mathrm{f}$ and $\Delta \mathrm{D}$ shifts of around $-13.7 \pm 0.2 \mathrm{~Hz}$ and $5.2 \pm 0.2 \times 10^{-6}$, respectively, suggesting smaller membrane effects. At $\mathrm{q}=2.5$, the membrane activity diminished further and the resulting $\Delta \mathrm{f}$ and $\Delta \mathrm{D}$ shifts were around $-7.0 \pm 0.7 \mathrm{~Hz}$ and $4.2 \pm 0.4 \times 10^{-6}$, respectively. The buffer washing step removed most bicelles from SLBs in the cases of $q=0.25$ and 2.5, 
as indicated by the final $\Delta \mathrm{f}$ shifts returning to baseline values while non-zero final $\Delta \mathrm{D}$ shifts suggest SLBs become less rigid upon interaction with bicelles. By contrast, some bicelles remained in SLB in the case of $\mathrm{q}=0.05$, as evidenced by the final $\Delta \mathrm{f}$ and $\Delta \mathrm{D}$ shifts that were smaller and greater, respectively, than baselines. Taken together, the results show that bicelles have appreciable activity at $\mathrm{q}=0.05$, moderate activity at $\mathrm{q}=0.25$ and minor activity at $\mathrm{q}=2.5$. This trend supports that DOPC likely interferes with GML activity in a concentration-dependent manner since GML concentration is constant in all q-ratios; the higher the DOPC concentration, the smaller the effects.
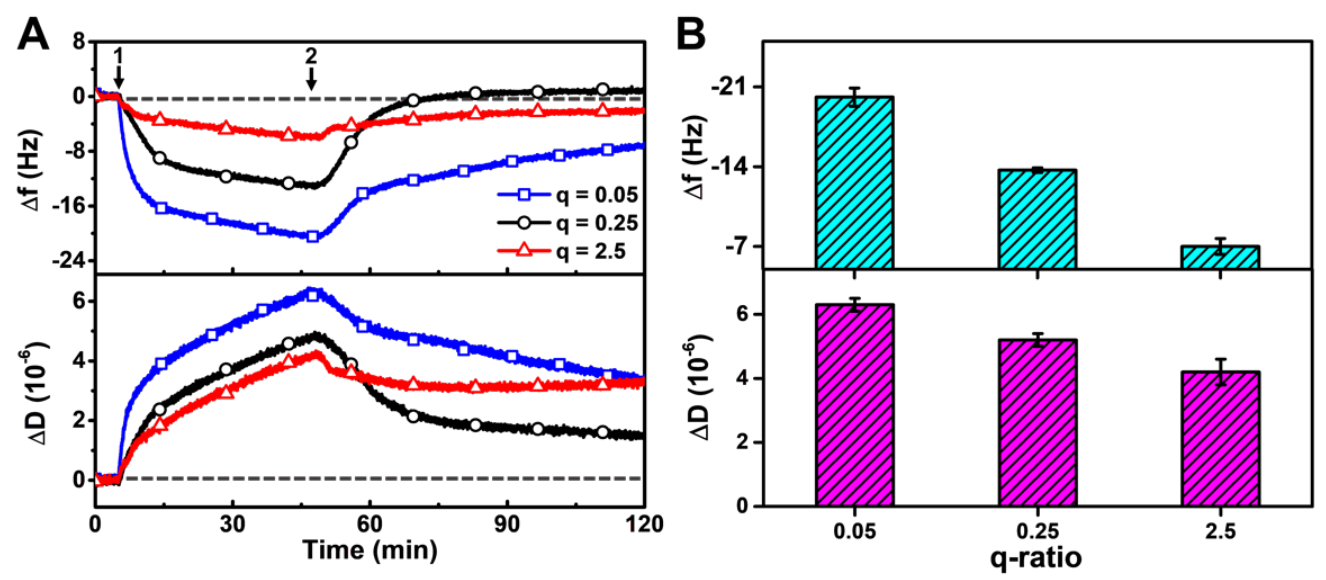

Figure 6.4. Characterization of bicelle-SLB interactions at different q-ratios. The GML concentration in DOPC/GML bicelles at different q-ratios was fixed at $0.125 \mathrm{mM}$. (A) Frequency and dissipation shifts, $\Delta \mathrm{f}$ (upper panel) and $\Delta \mathrm{D}$ (lower panel), respectively, were recorded as a function of time. The zero baselines, as denoted by dotted lines, correspond to pre-fabricated SLBs. Arrow (1) indicates the time point of bicelle injection and Arrow (2) buffer wash. (B) Column graphs of peak shifts (upper panel for $\Delta \mathrm{f}$ and lower panel for $\Delta \mathrm{D}$ ).

\subsubsection{Interaction with $S$. aureus Bacteria}

Subsequently, the biological activity of bicelles against $S$. aureus was tested with MBC assay and Live/Dead bacterial viability experiment using CFU enumeration technique for bicelles at $\mathrm{q}=0.25$. Prior to conducting the antibacterial assessments, the DOPC/GML bicelle formulations were characterized for the structure and size by employing cryo-TEM. 
Based on knowledge from biophysical evaluations above, $q=0.25$ was chosen for all biological evaluations with the observed moderate membrane-disruptive activity on prefabricated SLBs by QCM-D and more importantly, the fluorescence microscopy results showed pore formation at the q-value, which suggests potent antibacterial mechanism. On the other hand, although $\mathrm{q}=0.05$ bicelles had greater activity, they only caused phase separation instead of pores, which suggests less antibacterial potency. Bicelles with $\mathrm{q}=2.5$ showed minor activity as well as no phase separation or pore formation. Altogether, the condition of DOPC/GML bicelles at $\mathrm{q}=0.25$ were most promising candidate for antibacterial activity and it was adopted for the biological experiments.

\section{Bicellar Formulation Characterization}

Cyro-TEM was used to characterize the shape of DOPC/GML bicelles at the condition of containing potential of biological activity at $\mathrm{q}=0.25$. As presented in Figure 6.5A, a number of circular structures were observed with size distributed around $100-600 \mathrm{~nm}$. The circular vesicle-shaped structure was also reported in a previous study with DOPC/DHPC bicelles ${ }^{76}$ and agreed well with bicelle sizes obtained from dynamic light scattering with size averaged around $479 \pm 116 \mathrm{~nm}$ as shown in the Cyro-TEM micrographs. Later it was noticed that the circular structure reported for DOPC/DHPC bicelles ${ }^{76}$ was observed at a higher q-ratio where DOPC concentration was 3.5 times more than DHPC concentration and thus vesicle formation as dominated by DOPC is likely more favorable. By contrast, the circular structure observed in this study with DOPC/GML bicelles is probably due to formation of mixed micelles afforded by GML-dominated system where GML concentration was 4 times higher DOPC concentration and hence micellar shape is more probable.

\section{Bactericidal Activity}

Antibacterial activity of DOPC/GML bicelles were assessed with colony-forming unit (CFU) enumeration assay, which allows to determine lowest concentration of the bicelle at which no bacterial cell viability were observed with $99.99 \%$ killing $S$. aureus and the 
lowest bactericidal concentration is defined as MBC value. As presented in Figure 6.5B, the treatment with $0.031 \mathrm{mM}$ GML in DOPC/GML bicelle at $\mathrm{q}=0.25$ led to slightly decreased cell viability around $1 \times 10^{5} \mathrm{CFU} \mathrm{mL}^{-1}$ compared to control bacterial cell density at $5 \times 10^{5} \mathrm{CFU} \mathrm{mL}^{-1}$. At $0.063 \mathrm{mM} \mathrm{GML}$, significant reduced bacterial cell viability were

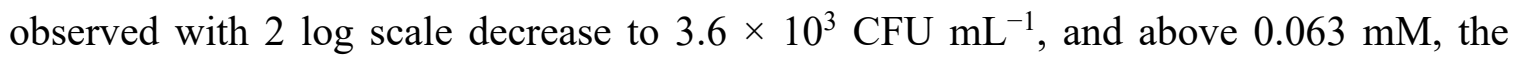
bacterial viability is not detectable so that the MBC value of the DOPC/GML bicelle was recorded at $0.125 \mathrm{mM}$. The value is well supported by anti-S. aureus activity of free GML samples $(31-63 \mu \mathrm{M})^{62}$. 


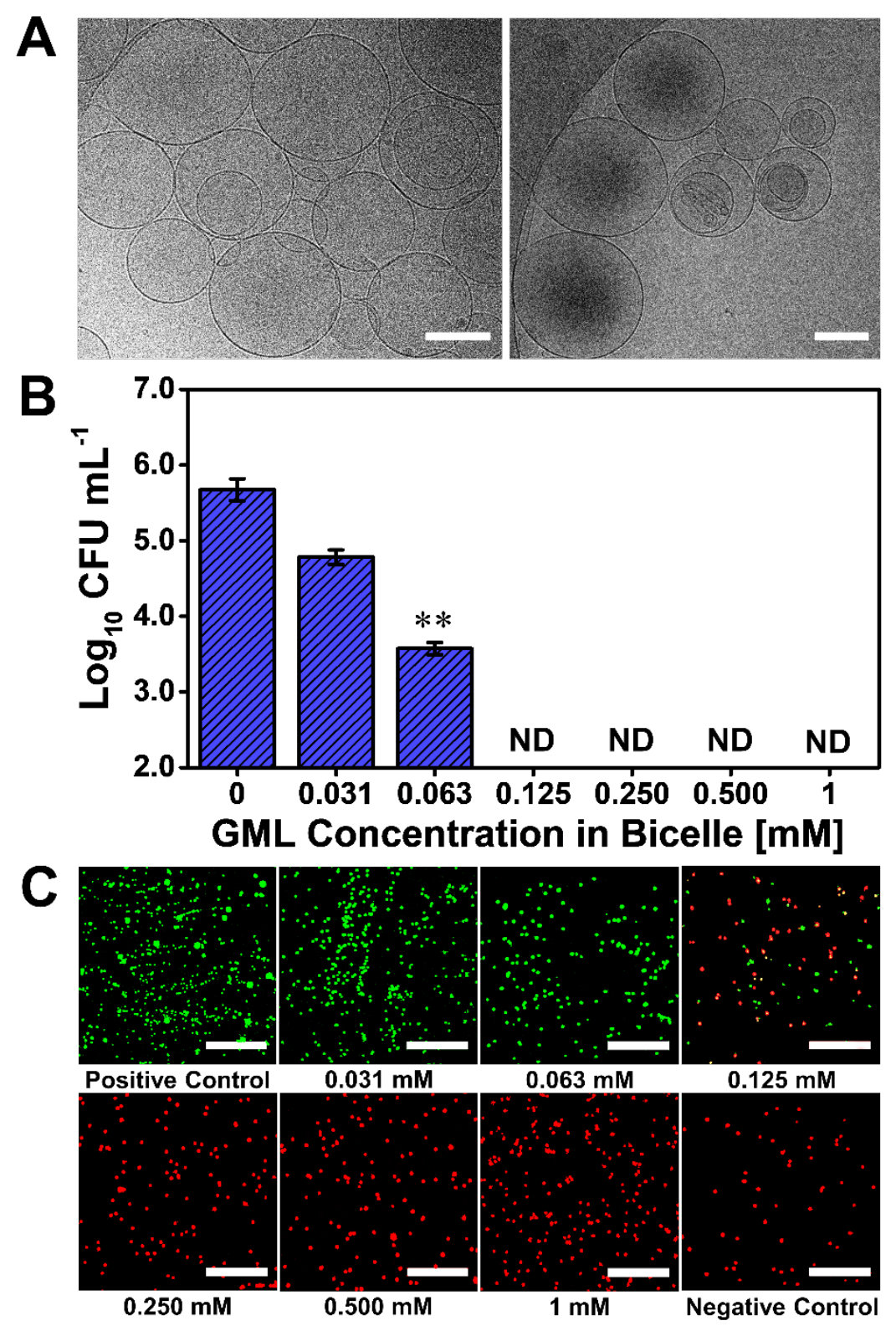

Figure 6.5. Biological activity evaluation of GML/DOPC bicelle at $q=0.25$. (A) Cryo-TEM micrographs of DOPC/GML bicellar formulation at fixed GML concentration of $0.125 \mathrm{mM}$ in the bicelle. The circular structure of the bicelles were detected in a size range of $100-600 \mathrm{~nm}$. Scale bars are $200 \mu \mathrm{m}$. (B) Antibacterial effect of DOPC/GML bicelles against $S$. aureus. The control bacterial cell concentration was $5 \times 10^{5} \mathrm{CFU} \mathrm{mL}^{-1}$ and $\mathrm{MBC}$ value was $0.125 \mathrm{mM}$, indicating that the lowest GML concentration in the bicelle at which no alive bacterial cells are observed (marked as ND, not determined). Mean \pm standard deviation was collected from $\mathrm{n}=3$ experiments. (C) Livedead bacterial viability evaluation of DOPC/GML bicelle-treated $S$. aureus cells at vary GML concentration in bicelles by confocal laser scanning microscopy (CLSM) imaging. The control 
bacterial cell concentration was $1 \times 10^{7} \mathrm{CFU} \mathrm{mL} \mathrm{mL}^{-1}$. Live and dead bacterial cells were detected in green and red stain, respectively. Representative images are presented of triplicated experiments and scale bars are $50 \mu \mathrm{m}$.

\section{Microscopic Observation of Bactericidal Effect}

In order to confirm that bacterial cell killing effect of the DOPC/GML bicelle at condition corresponding to $\mathrm{MBC}$ assay, confocal microscopic imaging was carried out for Live-Dead bacterial viability evaluation of DOPC/GML bicelle on S. aureus. The live and dead bacterial cells were stained with green the green SYTO 9 dye and the red PI dye which are able to permeate the alive or dead bacterial cell membranes ${ }^{77}$, respectively. To visualize using the microscopy, 20-fold higher cell numbers at $1 \times 10^{7} \mathrm{CFU} \mathrm{mL}^{-1}$ (high cell numbers are required for imaging) were incubated with DOPC/GML bicelle at $\mathrm{q}=0.25$ and then the bacterial cell stain was processed. As shown in Figure 6.5C, a positive control comprising untreated $S$. aureus cells showed the green stain of all live cells. However, as the GML concentration in the bicelle increased from 0.031 to $0.125 \mathrm{mM}$, the green stain decreased, indicating the decrease in the number of live bacterial cells. At $0.25 \mathrm{mM}$ GML and above, including the negative control, there was red stain of all dead cells, which suggests complete killing caused from bactericidal effect of the DOPC/GML bicelle samples. The results demonstrated that DOPC/GML bicelles can kill $S$. aureus bacteria at $\mathrm{q}=0.25$.

\section{Cell Viability and Cytotoxicity Testing}

To assess the safeness of the DOPC/GML bicelles for relevant application such as skincare formulations, cell viability and cytotoxicity test were performed over skin cells (HaCaT cell line). Human immortalized keratinocytes $(\mathrm{HaCaT})$ are a nontumorigenic cell line from epidermal skin cells, adapted for long-term growth ${ }^{78,79}$. The occupied colorimetric assay was the cell counting kit-8 (CCK-8) (Dojindo Molecular Technologies, Rockville, MD). The compound, WST-8 [2-(2-methoxy-4-nitrophenyl)-3-(4-nitrophenyl)-5-(2,4disulfophenyl)-2H-tetrazolium, monosodium salt] is reduced by dehydrogenase in cells to produce formazan ${ }^{80}$. The product of the reaction is seen with the naked eye by the color 
change of the culture medium to orange. The number of live cells is directly proportional to the amount of formazan stain ${ }^{81,82}$. For this experiment tree concentration of the DOPC/GML bicelles were gauged $(0.5 \mathrm{mM}$ to $2 \mathrm{mM})$. The bicelles were toxic to the cells at $1 \mathrm{mM}$ and $2 \mathrm{mM}$, no toxicity was evidenced with $0.5 \mathrm{mM}$ (Figure 6.6.). GML alone, at the same concentrations, was used as control and the results were similar (data not shown). The results match perfectly with other outcomes found in the literature, where GML caused no toxicity on mammalian cells at $100 \mathrm{ug} / \mathrm{ml}(0.5 \mathrm{mM})^{83}$. This data allows to support the safeness of DOPC/GML bicelles on skin cells at concentrations below $0.5 \mathrm{mM} \mathrm{GML}$, and their feasible use on skin models in vivo or in situ for further evaluation.

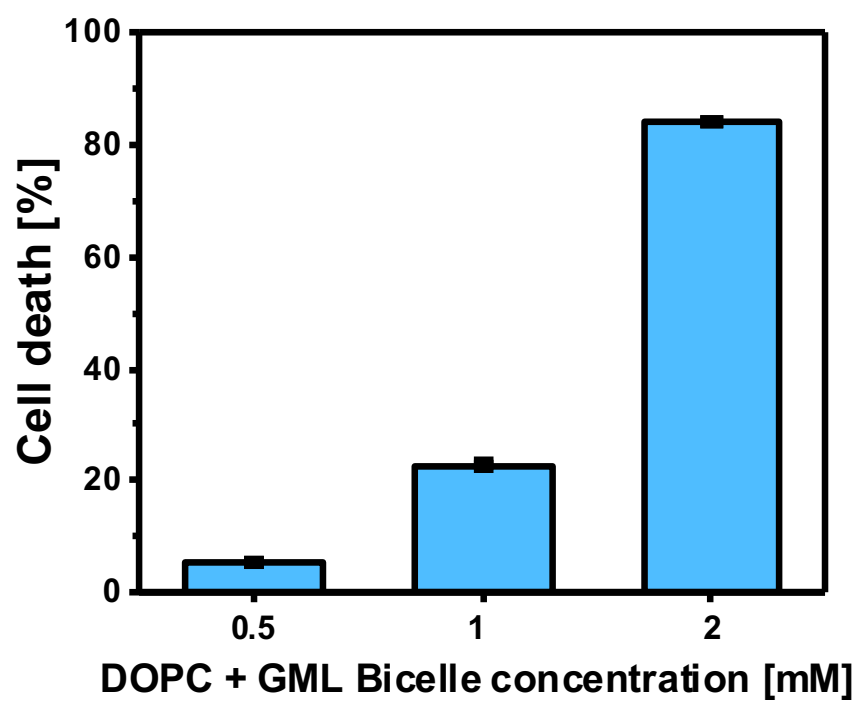

Figure 6.6. Cytotoxicity testing of HaCaT cell line. The immortalized keratinocytes were treated with different concentration of the DOPC/GML bicelles and incubated for 72hrs, after which the CCK reagent was added to the cells for $1 \mathrm{hr}$ incubation. The colorimetric culture media absorbance was measured with a plate reader at $450 \mathrm{~nm}$. The $0.5 \mathrm{mM}$ GML concentration presented $6 \%$ of cell death, with $1 \mathrm{mM} \mathrm{23 \%}$ and with $2 \mathrm{mM} \mathrm{89 \%}$. Mean \pm standard deviation was collected from $\mathrm{n}=3$ experiments.

\subsection{Conclusion}

With this investigations it was demonstrated the applications of DOPC/GML bicelles for SLB fabrication and antibacterial activity. The QCM-D and fluorescence microscopy 
techniques were used complementarily to characterize the bicelle adsorption and SLB formation. The results reveal that only $\mathrm{q}=2.5$ is suitable for SLB formation and the best conditions are at $\geq 0.063 \mathrm{mM}$ DOPC where GML concentrations are low and SLB formation is relatively fast. The membrane-disruptive activity of bicelles with prefabricated SLBs was also gauged by conducting QCM-D experiments, as well as with $S$. aureus bacteria by MBC and Live/Dead bacterial cell viability experiments. The safeness of the bicellar systems was verified with cytotoxicity tests. Bicelles had different levels of membrane activity against prefabricated SLBs at different q-ratios given a fixed GML concentration, i.e., activity depended on DOPC concentration. Collectively, these results further provided the condition for antibacterial testing against $S$. aureus bacteria, which is $\mathrm{q}=0.25$ and proved suitable for antibacterial applications; aside from being safe on keratinocyte cells at GML concentrations below $0.5 \mathrm{mM}$. Altogether, the findings show the possibility of using GML as a bicelle component for SLB fabrication and antibacterial applications, as well as engineering phospholipid-monoglyceride bicelles for relevant applications such as in skincare formulations. 


\section{Reference}

1 Marcotte, I. \& Auger, M. Bicelles as model membranes for solid-and solution-state NMR studies of membrane peptides and proteins. Concepts in Magnetic Resonance Part A 24, 17-37 (2005).

2 Ram, P. \& Prestegard, J. Magnetic field induced ordering of bile salt/phospholipid micelles: new media for NMR structural investigations. Biochimica et Biophysica Acta Biomembranes 940, 289-294 (1988).

$3 \mathrm{Hu}$, A. et al. Lipid-based nanodiscs as models for studying mesoscale coalescencea transport limited case. Soft matter 10, 5055-5060 (2014).

4 Dürr, U. H., Gildenberg, M. \& Ramamoorthy, A. The magic of bicelles lights up membrane protein structure. Chemical reviews 112, 6054-6074 (2012).

5 Caldwell, T. A. et al. Low-q bicelles are mixed micelles. The journal of physical chemistry letters 9, 4469-4473 (2018).

6 Nieh, M.-P. et al. Magnetically alignable phase of phospholipid "bicelle" mixtures is a chiral nematic made up of wormlike micelles. Langmuir 20, 7893-7897 (2004).

7 Katsaras, J., Harroun, T. A., Pencer, J. \& Nieh, M.-P. "Bicellar" lipid mixtures as used in biochemical and biophysical studies. Naturwissenschaften 92, 355-366 (2005).

8 Harroun, T. A. et al. Comprehensive examination of mesophases formed by DMPC and DHPC mixtures. Langmuir 21, 5356-5361 (2005).

9 van Dam, L., Karlsson, G. \& Edwards, K. Morphology of magnetically aligning DMPC/DHPC aggregates perforated sheets, not disks. Langmuir 22, 3280-3285 (2006).

10 Pabst, G., Kučerka, N., Nieh, M.-P., Rheinstädter, M. \& Katsaras, J. Applications of neutron and X-ray scattering to the study of biologically relevant model membranes. Chemistry and Physics of Lipids 163, 460-479 (2010).

11 Nieh, M.-P. et al. Temperature driven annealing of perforations in bicellar model membranes. Langmuir 27, 4838-4847 (2011).

12 Li, M. et al. Morphological characterization of DMPC/CHAPSO bicellar mixtures: a combined SANS and NMR study. Langmuir 29, 15943-15957 (2013).

13 Vold, R. R., Prosser, R. S. \& Deese, A. J. Isotropic solutions of phospholipid bicelles: a new membrane mimetic for high-resolution NMR studies of polypeptides. Journal of biomolecular NMR 9, 329-335 (1997). 
14 van Dam, L., Karlsson, G. \& Edwards, K. Direct observation and characterization of DMPC/DHPC aggregates under conditions relevant for biological solution NMR. Biochimica et Biophysica Acta (BBA)-Biomembranes 1664, 241-256 (2004).

15 Dürr, U. H., Soong, R. \& Ramamoorthy, A. When detergent meets bilayer: birth and coming of age of lipid bicelles. Progress in nuclear magnetic resonance spectroscopy 69, 1 (2013).

16 De Angelis, A. A. \& Opella, S. J. Bicelle samples for solid-state NMR of membrane proteins. Nature protocols 2, 2332 (2007).

17 Lin, L. et al. Hybrid bicelles as a $\mathrm{pH}$-sensitive nanocarrier for hydrophobic drug delivery. RSC advances 6, 79811-79821 (2016).

18 Liu, Y., Xia, Y., Rad, A. T., Aresh, W. \& Nieh, M.-P. in Liposomes 273-282 (Springer, 2017).

19 Lin, L. et al. Doxorubicin and indocyanine green loaded hybrid bicelles for fluorescence imaging guided synergetic chemo/photothermal therapy. Bioconjugate chemistry 28, 2410-2419 (2017).

20 Uchida, N., Nishizawa Horimoto, N., Yamada, K., Hikima, T. \& Ishida, Y. Kinetically stable bicelles with dilution tolerance, size tunability, and thermoresponsiveness for drug delivery applications. ChemBioChem 19, 1922-1926 (2018).

21 Barbosa-Barros, L. et al. Effect of bicellar systems on skin properties. International journal of pharmaceutics 352, 263-272 (2008).

22 Barbosa-Barros, L. et al. Penetration and growth of DPPC/DHPC bicelles inside the stratum corneum of the skin. Langmuir 24, 5700-5706 (2008).

23 Rodriguez, G. et al. Conformational changes in stratum corneum lipids by effect of bicellar systems. Langmuir 25, 10595-10603 (2009).

24 Rubio, L. et al. Bicellar systems for in vitro percutaneous absorption of diclofenac. International journal of pharmaceutics 386, 108-113 (2010).

25 Rodríguez, G. et al. Bicellar systems as modifiers of skin lipid structure. Colloids and Surfaces B: Biointerfaces 84, 390-394 (2011).

26 Rubio, L. et al. Bicellar systems as a new colloidal delivery strategy for skin. Colloids and Surfaces B: Biointerfaces 92, 322-326 (2012). 
27 Barbosa-Barros, L. et al. Bicelles: lipid nanostructured platforms with potential dermal applications. Small 8, 807-818 (2012).

28 Rubio, L. et al. Bicellar systems as new delivery strategy for topical application of flufenamic acid. International journal of pharmaceutics 444, 60-69 (2013).

29 Rodríguez, G. et al. Bicelles: new lipid nanosystems for dermatological applications. Journal of biomedical nanotechnology 11, 282-290 (2015).

30 Sackmann, E. Supported membranes: scientific and practical applications. Science 271, 43-48 (1996).

31 Hardy, G. J., Nayak, R. \& Zauscher, S. Model cell membranes: Techniques to form complex biomimetic supported lipid bilayers via vesicle fusion. Current Opinion in Colloid \& Interface Science 18, 448-458 (2013).

32 Richter, R. P., Bérat, R. \& Brisson, A. R. Formation of solid-supported lipid bilayers: an integrated view. Langmuir 22, 3497-3505 (2006).

33 Jackman, J. A., Ferhan, A. R. \& Cho, N.-J. Surface-based nanoplasmonic sensors for biointerfacial science applications. Bulletin of the Chemical Society of Japan 92, 14041412 (2019).

34 Jackman, J. A. et al. Materials Nanoarchitectonics for Mechanical Tools in Chemical and Biological Sensing. Chemistry-An Asian Journal 13, 3366-3377 (2018).

35 Castellana, E. T. \& Cremer, P. S. Solid supported lipid bilayers: From biophysical studies to sensor design. Surface Science Reports 61, 429-444 (2006).

36 Jackman, J. A., Ferhan, A. R. \& Cho, N.-J. Nanoplasmonic sensors for biointerfacial science. Chemical Society Reviews 46, 3615-3660 (2017).

37 Mashaghi, A. et al. Label-free characterization of biomembranes: from structure to dynamics. Chemical Society Reviews 43, 887-900 (2014).

38 Öncel, M. Ö. Ö., Garipcan, B. \& Inci, F. in Biomimetic Lipid Membranes: Fundamentals, Applications, and Commercialization 193-212 (Springer, 2019).

39 Soler, M. et al. Two-dimensional label-free affinity analysis of tumor-specific CD8 T cells with a biomimetic plasmonic sensor. ACS sensors 3, 2286-2295 (2018).

40 Wagh, P. \& Escobar, I. C. Biomimetic and bioinspired membranes for water purification: A critical review and future directions. Environmental Progress \& Sustainable Energy 38, e13215 (2019). 
41 Nikoleli, G.-P., Nikolelis, D., Siontorou, C. G. \& Karapetis, S. Lipid membrane nanosensors for environmental monitoring: The art, the opportunities, and the challenges. Sensors 18, 284 (2018).

42 Nikoleli, G.-P. Advanced lipid based biosensors for food analysis. Advances in Food and Nutrition Research 91, 301 (2020).

43 Belling, J. N. et al. Lipid Bicelle Micropatterning Using Chemical Lift-Off Lithography. ACS applied materials \& interfaces 12, 13447-13455 (2020).

44 Zeineldin, R. et al. Using bicellar mixtures to form supported and suspended lipid bilayers on silicon chips. Langmuir 22, 8163-8168 (2006).

45 Tabaei, S. R., Jönsson, P., Brändén, M. \& Höök, F. Self-assembly formation of multiple DNA-tethered lipid bilayers. Journal of structural biology 168, 200-206 (2009).

46 Morigaki, K., Kimura, S., Okada, K., Kawasaki, T. \& Kawasaki, K. Formation of substrate-supported membranes from mixtures of long-and short-chain phospholipids. Langmuir 28, 9649-9655 (2012).

47 Saleem, Q., Zhang, Z., Petretic, A., Gradinaru, C. C. \& Macdonald, P. M. Single lipid bilayer deposition on polymer surfaces using bicelles. Biomacromolecules 16, 10321039 (2015).

48 Yamada, N. L., Sferrazza, M. \& Fujinami, S. In-situ measurement of phospholipid nanodisk adhesion on a solid substrate using neutron reflectometry and atomic force microscopy. Physica B: Condensed Matter 551, 222-226 (2018).

49 Kolahdouzan, K. et al. Optimizing the formation of supported lipid bilayers from bicellar mixtures. Langmuir 33, 5052-5064 (2017).

50 Sut, T. N., Jackman, J. A. \& Cho, N.-J. Understanding How Membrane Surface Charge Influences Lipid Bicelle Adsorption onto Oxide Surfaces. Langmuir 35, 8436-8444 (2019).

51 Sut, T. N. et al. Influence of $\mathrm{NaCl}$ Concentration on Bicelle-Mediated SLB Formation. Langmuir 35, 10658-10666 (2019).

52 Sut, T. N., Park, S., Choe, Y. \& Cho, N.-J. Characterizing the Supported Lipid Membrane Formation from Cholesterol-Rich Bicelles. Langmuir 35, 15063-15070 (2019). 53 Keller, C., Glasmästar, K., Zhdanov, V. \& Kasemo, B. Formation of supported membranes from vesicles. Physical Review Letters 84, 5443 (2000). 
54 Jackman, J. A. \& Cho, N.-J. Supported Lipid Bilayer Formation: Beyond Vesicle Fusion. Langmuir 36, 1387-1400, doi:10.1021/acs.langmuir.9b03706 (2020).

55 Sut, T. N., Park, S., Yoon, B. K., Jackman, J. A. \& Cho, N.-J. Supported Lipid Bilayer Formation from Phospholipid-Fatty Acid Bicellar Mixtures. Langmuir, doi:10.1021/acs.langmuir.0c00675 (2020).

56 Sut, T. N., Park, S., Yoon, B. K., Jackman, J. A. \& Cho, N.-J. Optimal Formation of Uniform-Phase Supported Lipid Bilayers from Phospholipid-Monoglyceride Bicellar Mixtures. Journal of Industrial and Engineering Chemistry (2020).

57 Hauser, H. Short-chain phospholipids as detergents. Biochimica et Biophysica Acta (BBA)-Biomembranes 1508, 164-181 (2000).

58 Sanders, C. R. \& Prestegard, J. Magnetically orientable phospholipid bilayers containing small amounts of a bile salt analogue, CHAPSO. Biophysical journal 58, 447460 (1990).

59 McKibbin, C. et al. Opsin stability and folding: modulation by phospholipid bicelles. Journal of molecular biology 374, 1319-1332 (2007).

60 Kabara, J. J., Swieczkowski, D. M., Conley, A. J. \& Truant, J. P. Fatty acids and derivatives as antimicrobial agents. Antimicrobial agents and chemotherapy 2, 23-28 (1972).

61 Conley, A. J. \& Kabara, J. J. Antimicrobial action of esters of polyhydric alcohols. Antimicrobial agents and chemotherapy 4, 501-506 (1973).

62 Schlievert, P. M. \& Peterson, M. L. Glycerol monolaurate antibacterial activity in broth and biofilm cultures. PloS one 7 (2012).

63 Li, Q. et al. Glycerol monolaurate prevents mucosal SIV transmission. Nature 458, 1034-1038 (2009).

64 Yoon, B. K., Jackman, J. A., Kim, M. C. \& Cho, N.-J. Spectrum of membrane morphological responses to antibacterial fatty acids and related surfactants. Langmuir 31, 10223-10232 (2015).

65 Jackman, J. A., Yoon, B. K., Li, D. \& Cho, N.-J. Nanotechnology formulations for antibacterial free fatty acids and monoglycerides. Molecules 21, 305 (2016). 
66 Cho, N.-J., Frank, C. W., Kasemo, B. \& Höök, F. Quartz crystal microbalance with dissipation monitoring of supported lipid bilayers on various substrates. nature protocols 5, 1096-1106 (2010).

67 Valle-González, E. R. et al. Characterizing How Acidic pH Conditions Affect the Membrane-Disruptive Activities of Lauric Acid and Glycerol Monolaurate. Langmuir 34, $13745-13753$ (2018).

68 Zhang, M. S., Sandouk, A. \& Houtman, J. C. Glycerol Monolaurate (GML) inhibits human T cell signaling and function by disrupting lipid dynamics. Scientific reports $\mathbf{6}$, 30225 (2016).

69 Zhang, M. S. et al. Glycerol monolaurate induces filopodia formation by disrupting the association between LAT and SLP-76 microclusters. Sci. Signal. 11, eaam9095 (2018). 70 Oliveira, T. R., Benatti, C. R. \& Lamy, M. T. Structural characterization of the interaction of the polyene antibiotic amphotericin B with DODAB bicelles and vesicles. Biochimica et Biophysica Acta (BBA)-Biomembranes 1808, 2629-2637 (2011).

71 Dittmer, J. et al. Incorporation of antimicrobial peptides into membranes: a combined liquid-state NMR and molecular dynamics study of alamethicin in DMPC/DHPC bicelles. The journal of physical chemistry B 113, 6928-6937 (2009).

72 Whiles, J. A. et al. Orientation and effects of mastoparan X on phospholipid bicelles. Biophysical journal 80, 280-293 (2001).

73 Bortolus, M., Dalzini, A., Toniolo, C., Hahm, K. S. \& Maniero, A. L. Interaction of hydrophobic and amphipathic antimicrobial peptides with lipid bicelles. Journal of Peptide Science 20, 517-525 (2014).

74 Raja, Z. et al. Insight into the mechanism of action of temporin-SHa, a new broadspectrum antiparasitic and antibacterial agent. PLoS One 12 (2017).

75 Mamusa, M., Salvatore, A. \& Berti, D. Structural Modifications of DPPC Bilayers upon Inclusion of an Antibacterial Cationic Bolaamphiphile. Langmuir 34, 8952-8961 (2018).

76 Rodríguez, G. et al. A unique bicellar nanosystem combining two effects on stratum corneum lipids. Molecular pharmaceutics 9, 482-491 (2012). 
77 Robertson, J., McGoverin, C., Vanholsbeeck, F. \& Swift, S. Optimisation of the protocol for the LIVE/DEAD BacLight Bacterial Viability Kit for rapid determination of bacterial load. Frontiers in Microbiology 10, 801 (2019).

78 Schürer, N., Köhne, A., Schliep, V., Barlag, K. \& Goerz, G. Lipid composition and synthesis of HaCaT cells, an immortalized human keratinocyte line, in comparison with normal human adult keratinocytes. Experimental dermatology 2, 179-185 (1993).

79 Micallef, L. et al. Effects of extracellular calcium on the growth-differentiation switch in immortalized keratinocyte $\mathrm{HaCaT}$ cells compared with normal human keratinocytes. Experimental dermatology 18, 143-151 (2009).

80 (Dojindo Molecular Technologies, Inc, 2016).

81 Ishiyama, M., Miyazono, Y., Sasamoto, K., Ohkura, Y. \& Ueno, K. A highly watersoluble disulfonated tetrazolium salt as a chromogenic indicator for NADH as well as cell viability. Talanta 44, 1299-1305 (1997).

82 Tominaga, H. et al. A water-soluble tetrazolium salt useful for colorimetric cell viability assay. Analytical Communications 36, 47-50 (1999).

83 Peterson, M. L. \& Schlievert, P. M. Glycerol Monolaurate Inhibits the Effects of Gram-Positive Select Agents on Eukaryotic Cells. Biochemistry 45, 2387-2397, doi:10.1021/bi051992u (2006). 


\section{Chapter 7}

\section{Conclusion and Future Work}

This chapter links all the concluding remarks and clarifies how each one fulfilled a milestone of the thesis hypothesis. It outlines the analytical framework designed to test the hypothesis and the resulting key findings to prove it. The implications and impact of the study about antimicrobial lipids, glycolic acid and the bicelles for the treatment of acne vulgaris and atopic dermatitis are discussed in deep. At last, the opportunities and the possible working strategies for this research in the future are recounted. 


\subsection{Discussion and Concluding Remarks}

The objective of this thesis was to develop a mild, yet effective treatment for acne vulgaris and atopic dermatitis using antimicrobial lipids to fabricate a bicelle and use it in combination with glycolic acid. Within this scope, particular attention was placed on the medium-chain saturated antimicrobial lipids, lauric acid and glycerol monolaurate, and the hydroxy acid, glycolic acid. An ample analytical framework was established to: 1) characterize antimicrobial lipids and determine their potency against $C$. acnes under skinlike $\mathrm{pH}$ conditions, 2) resolve the potency of glycolic acid, also in skin-like acidic settings, and its mechanism of action against C. acnes, 3 ) assess the potency of glycerol monolaurate assembled as a bicelle to inhibit the growth of $S$. aureus and 4) verify the safeness, target delivery and protection of GML as a bicelle. The key findings based on the analytical framework are outlined below:

1. Glycerol monolaurate was the most potent medium-chain saturated antimicrobial lipid against $C$. acnes bacteria in all $\mathrm{pH}$ conditions.

The results for the first set of experiments, encompassed in chapter 4, showed that the monoglyceride was the most stable under a broad range of $\mathrm{pH}$; that it induced membrane budding in a solution-phase model membrane platform; and it was the most potent against C. acnes bacteria. The CMC evaluations of the anionic fatty acid, lauric acid, decreased dramatically under acidic skin-like $\mathrm{pH}$ (4.5) in comparison with the often tested physiological $\mathrm{pH}$ (7.4). The lessening in CMC occurred because the majority of lauric acid molecules were protonated under acidic conditions and hence micellar aggregation became more thermodynamically favorable. In the case of GML, the CMC did not change under acidic conditions, proving the lack of $\mathrm{pH}$ dependence because of its nonionic nature. The assessment with the SLB showed that the monoglyceride induced a different morphological responses in comparison to LA. Lauric acid caused tubule formation of the bilayer at almost neutral $\mathrm{pH}$ and membrane phase separation under acidic conditions. Glycerol monolaurate provoked membrane budding in both $\mathrm{pH}$ settings, with the only difference in bigger size with low $\mathrm{pH}$. Regarding to the anti-infective testing, a higher growth inhibition of $C$. acnes 
was exhibited with glycerol monolaurate. The monoglycerides' nonionic protonated nature and its membrane budding behavior contributed to disrupt the bacterial cell membrane with lower concentration than lauric acid. This mode of action has proven to leave a very small window for the development of bacteria resistance and makes it a viable solution for acne infections ${ }^{1-3}$. Overall, it was demonstrated that the $\mathrm{pH}$ governs the protonation state and hence the self-assemble and antibacterial activity of the molecule. Unlike previous data, the fact that antimicrobial lipids were active against SLB at concentrations above the CMC value does not necessarily correlated with the minimum inhibitory concentration.

2. Glycolic acid exhibited potent antibacterial activity against $C$. acnes and the mechanistic activity was by membrane disruption.

Chapter 5, containing the second set of results, detailed how glycolic acid exhibited powerful anti-infective activity against $C$. acnes, especially in acidic $\mathrm{pH}$ environments around $\mathrm{pH} 3$ to 4.5. Conventionally, glycolic acid is used at rather high concentrations (50$70 \%$ ) in chemical peeling treatments, however, it was revealed that it could inhibit bacterial growth at concentrations as lower as $0.2 \% \mathrm{w} / \mathrm{w}$ with $\mathrm{pH}$ conditions that were appropriate for over-the-counter formulation development. The mechanistic behavior of glycolic acid against $C$. acnes bacteria was defined to be by cell membrane disruption and the potency relied on the solution $\mathrm{pH}$. The data indicated that glycolic acid was more active when it existed in the nonionic (protonated) form, which was consistent with a greater tendency of this form to partition into phospholipid membranes as compared to the anionic (deprotonated) form. The membrane disruptive mechanism of action, is particularly significant because there is a documented higher barrier to bacterial resistance of membrane-active antibacterial drugs, like antimicrobial lipids, and the same probabilities might apply for glycolic acid as well.

3. The potency of GML mixed with the bicelle decreased, nonetheless it remained within acceptable concentrations.

In the last chapter of results, chapter 6 , it was determined that the bactericidal concentration 
of glycerol monolaurate mixed with the bicelle increased but the mechanistic activity remained the same; the size of the bicellar system was small enough to penetrate the skin appendages; and that the cytotoxicity assessment with skin cells was satisfactory. The minimum inhibitory and bactericidal concentrations of GML alone against $S$. aureus bacteria were $0.063 \mathrm{mM}$ and $0.125 \mathrm{mM}$, respectively. However, when the antimicrobial lipid was prepared with the phospholipid DOPC the value changed to $0.125 \mathrm{mM}$ for MIC and $0.250 \mathrm{mM}$ for $\mathrm{MBC}$. These concentrations are still within a good range and below the one from lauric acid alone of $1 \mathrm{mM}$ for MIC. The mechanistic activity of the bicelle was verified to cause membrane lysis, as with the monoglyceride unaccompanied. The morphology of the bicellar systems prepared with DOPC and glycerol monolaurate with qratio of 0.25 was multilamellar vesicle and the size was $\sim 470 \mathrm{~nm}$. The diameter of the skin appendages is $530 \mathrm{~nm}$, thus the bicelle can penetrate and deliver the monoglyceride in the target point. Another postulate is that the bicellar system can "sit" at the top of skin and slowly release the compound, protecting it from the environment. Nevertheless, in vivo and/or in situ tests were missing to corroborate all these assumptions. To verify the safeness of the bicelle and the monoglyceride alone, cell proliferation and viability tests were conducted with an immortalized keratinocyte line (HaCaT). The results showed a cell death percentage of less than $6 \%$ for both GML and the bicellar mixture with GML, validating the safeness of the delivery vehicle for cutaneous uptakes.

Together all the previous key findings allow to support most of the hypothesis affirming that: antimicrobial lipids assemble as bicelles and glycolic acid can be employed in additive multifunctional combination to treat $C$. acnes and $S$. aureus infections. First it was proven that glycerol monolaurate is the most potent saturated medium-chain antimicrobial lipid against $C$. acnes, as reported here, and $S$. aureus, as documented in numerous publications 3-7. Second, glycolic acid is capable to kill C. acnes bacteria at concentrations below overthe-counter products (normally 2 to $30 \%$ ) and the mechanistic behavior is by lysing the cell membrane. This is important since glycolic acid can treat the disease symptoms as well as modulate the skin microbiota by killing undesirable pathogens. Third, it was proven that GML assembled as a bicelle is a potent anti-infective agent against $S$. aureus, and its use on skin cells is safe. Unfortunately, time came to an end and the target delivery, penetration 
profile and protection of the monoglyceride inside of the bicellar system were not proven. Likewise the administration of glycerol monolaurate in combination with glycolic acid was missing. Despite of the lack of these tests, the use of glycerol monolaurate unaccompanied or with the bicelles, together with glycolic acid portrays as a viable solution for the skin conditions. The opportunities and strategies for future work are described in a section below.

\subsection{Implication and Impact}

The implication of this investigations is the feasible design of a direct therapeutic solution for acne vulgaris and atopic dermatitis that bypasses the need to use antibiotics or indirect approaches aimed at relieving disease symptoms alone. The main advantages are the killing effect of the bacteria causing the skin diseases, simultaneously the treatment of the symptoms and the avoidance of antibiotic resistance, all in one formulation. All these benefits would provide sustainable results and extend the time between possible relapsing periods. The usage of bicelles as the delivery method will allow for the release of the antimicrobial lipid in the skin appendages, where the microbiome dwells, and will protect GML from environmental degradation and enzymatic reactions. Another benefit of the use of nanocarriers, like bicellar systems, is the increase in shelf-life because of the higher stability ${ }^{8,9}$. Ultimate generation nanocarriers as bicosomes and bicellar systems protect bicelles from the change in morphology when diluted, retarding the highly undesirable effects of flocculation. Respecting to the bicelle composition, the phospholipid 1,2dioleoyl-sn-glycero-3-phosphocholine (DOPC) has proved to ease the application of other skin care formulations and moisturize skin ${ }^{10,11}$. Providing a good reference for the future experiment in situ with skin. As an indirect consequence the treatment might decrease the overall expenses, by reducing the number of purchased products and visits to the specialist.

The impact of the anti-infective activity of GML and glycolic acid can reach millions of people suffering from both diseases worldwide. Just for acne vulgaris the $35-100 \%$ of adolescents with the condition might benefit from the treatment ${ }^{12}$. In addition, the occurrence of acne in a large proportion of young adults might be limited. The emotional 
and mental negative impacts associated to the disease, as low self-esteem, poor social life, depression and anxiety might be equally tackled by improving the appearance of the patients and hence their confidence. Regarding to treatment expenses, the estimated expenditure of US\$3 billion dollars might be diminished by implementing this alternative therapy ${ }^{13,14}$. In the case of atopic dermatitis, the most favored would be the $25 \%$ of children globally accounted for this skin disorder ${ }^{15,16}$. Just in developed countries, like the United States, the medical bill for AD of approximately US\$442 million and the lost in productivity of around US\$314 million can be expect to decrease if the therapy continues to be developed and proves effective ${ }^{17}$. On top of the numbers and money spent, the help and relief it might provide to the patients, their families and caregivers would be priceless.

\subsection{Opportunities and Strategies}

As described, the designed analytical framework allowed to prove most of hypothesis, nonetheless, the target delivery, penetration profile and protection of the monoglyceride with the bicelle were not verified. Similarly the administration of glycerol monolaurate in combination with glycolic acid could not be tested. Henceforth, the main opportunities reside in the full assessment of the compounds in vivo and in situ. The initial strategy would be the in vivo evaluation of the bicelle with glycerol monolaurate and the monoglyceride alone to determine which one exerts a better outcome. Following the strategy of Rodríguez et al ${ }^{18}$ skin tissue, preferably from human or porcine skin, should be treated with PBS solutions containing the bicelles at a GML concentration of $1 \mathrm{mM}$, GML unaccompanied at the same concentration and the respective controls. In accordance with the protocol, skin disks of approximately $2.5 \mathrm{~cm}^{2}$ can be treated with the aforementioned samples by applying $\sim 15 \mu \mathrm{L}$ every hour four times. The skin tissues must be washed with deionized water after the addition of the samples and left to dry before continuing with the next application. The justification behind the $1 \mathrm{mM}$ GML concentration is the middle ground between the inhibitory value of $S$. aureus bacterium at $0.250 \mathrm{mM}$ and the toxicity of keratinocytes at 2 $\mathrm{mM}$. The treated skin disks must be analyzed with Cryo-TEM or as recommended in several publications with Freeze-Substitution TEM ${ }^{19,20}$, to determine the localization of the bicelles or the GML molecules in the stratum corneum — outermost layer of skin - 
Only electron microscopy techniques provide enough resolution to visualize the "actual" bicelle structure and the effect on the lipid mantle after the treatments. As highlighted before, it is expected that the bicelles do not penetrate into the interlamellar spaces of stratum corneum due to their large size; however, they must be able to infiltrate into the skin appendages — target delivery — and/or remain at the top and change the lipid organization. To evaluate the penetration profile and the structural changes on the lipid mantle other techniques as Attenuated Transmission Reflectance Fourier Transform Infrared Spectroscopy (ATR-FTIR) and X-ray Scattering can be applied. ATR-FTIR has been used to study the structure of bicelles, the thermal changes and the overall organization of the lipid mantle in the epidermis ${ }^{21}$. The permeation depth of the IR eases the study of the stratum corneum without the separation of the other skin layers and determines the lipid order phase according to the vibration frequency of their alkyl chains 22,23 . Highly organize lipids are in a gel phase, while less organize are in liquid-crystalline. If the results show that the lipid arrangement is in gel phase, the bicelles would have penetrated the corneocytes skin barrier and favor the organization of the lipids. On the opposite, if the lipids are in a liquid-crystalline phase, is probable that a large volume of the bicelles did not penetrate skin and remained at the top forming a protective lipid matrix 18. In a couple of publications using ATR-FTIR it has been validated that bicellar mixtures with DOPC adhere to the skin surface, protect the encapsulated compound and act as a drug reservoir by slowly releasing the drug ${ }^{18,24}$. As an orthogonal methodology, X-ray Scattering can be used to gain data regarding the supra-molecular lipid organization on skin tissues without crystallization ${ }^{25}$. An specific technique within X-ray Scattering, Scattering with Small Angle (SAXS), informs about the phase behavior and lamellarity ${ }^{21}$. If the bicelles deeply interact with skin and penetrate it the results will show a higher lipid order ${ }^{11}$. As with ATR-FTIR, if the skin tissue shows a lower lamellar order the bicelles sit at the top of skin. So far these techniques would permit to decipher which one, the bicelles with GML or the antibacterial lipid alone are more effective. It is very important to emphasize that the potential lack of effectiveness or improvement with the bicellar system delivery will lead to the fallback of encapsulating the antimicrobial lipid directly within a hydrogel. Hydrogels are "hydrophilic 3D-mensional networks, which are able to imbibe large amounts of water and resemble a biological tissue" ${ }^{26}$. They are used in numerous 
skincare preparations, like hydrogel mask to preserve moisture and keep the skin cells hydrated. At this point of the investigation the main goal would be to show proof-ofconcept results demonstrating the therapeutic efficacy of GML.

It is worth mentioning that glycerol monolaurate also possess a disadvantage when used against $S$. aureus bacteria. The bacterial cells secret an esterase enzyme that hydrolyses the ester linkages of the monoglyceride, favouring the separation of the building blocks and leading to the obtention of lauric acid and glycerol molecules ${ }^{27,28}$. As a result the potency of GML decreases and the effect is similar to the one of Lauric acid. To overcome this major drawback a potential solution implicates the use of 1-O-dodecyl-rac-glycerol (DDG), a derivative of lauric acid formed by a glycerol group linked to $\mathrm{C} 12$ with and ether bond instead of an ester as with GML. This LA derivative is not susceptible to hydrolysis and importantly, also exerts antibacterial activity against $S$. aureus albeit with different potency and mechanistic activity ${ }^{29,30}$. It would be necessary to verify if DDG is also active against C. acnes bacteria to consider it as a viable replacement for GML, nevertheless this would be a very last option, since the main objective is the use of GML and the bicellar system to protect it from the enzymatic reaction. Depending on the outcomes with GML, the bicellar system, the hydrogel and less possibly DDG the second stage or strategy can be tailored to assessed the cocktail effect of the antimicrobial lipid with glycolic acid. 
The testing with glycolic acid can involve the in vivo skin model or direct in situ trial with healthy volunteers. At this point the feasibility and safeness of the antimicrobial lipid treatment would have been gauged and glycolic acid is already known for being safely used over the counter in concentration below 30\%, therefore the trial with healthy volunteers seem reasonable. The bicelles or the hydrogel concentration should be tuned between 0.5 $\mathrm{mM}$ and $1 \mathrm{mM}$ GML, since the effect might be boost by glycolic acid. The concentration of the alpha hydroxy acid must be $\geq 2 \%$ because the results displayed bactericidal activity at concentrations above this percentage. The experimental design should follow a standard protocol for the evaluation of skincare products ${ }^{11,31,32}$. For example, 8 to 10 healthy volunteers might be selected for the trials and $20 \mu \mathrm{l}$ of the bicellar sample/hydrogel with glycolic acid should be applied in their forearm for a period of 10 days. Prior and during the treatment the volunteers should avoid the use of any product on the lower arm area. The typical biophysical measures for the in vivo evaluation of skincare products involve the transepidermal water loss (TEWL), skin hydration, melanin and erythema indexes and the elasticity parameters. The TEWL measures the water evaporation rates and is a good indicator of barrier function integrity ${ }^{33}$. The skin hydration and elasticity are a measure of water content, tonicity and extensibility ${ }^{34,35}$. The melanin and erythema index, are indicative of color change and irritation, respectively ${ }^{36}$. If all the listed parameters remain within acceptable levels in all the volunteers the therapy should be consider as a viable alternative to move on to the next trial with patients suffering from acne or atopic dermatitis. This last trial might be longer and more strenuous, but it would represent the final translational science step. The successful completion of all these trials will offer a new and effective therapy, with minimal secondary effects, longer relapsing periods and no concern for antibiotic resistance. All in one formulation, to hopefully improve the quality of life of millions of people. 


\section{References}

1 Desbois, A. P. \& Smith, V. J. Antibacterial free fatty acids: activities, mechanisms of action and biotechnological potential. Applied microbiology and biotechnology 85, 1629-1642 (2010).

2 Schlievert, P. M. \& Peterson, M. L. Glycerol monolaurate antibacterial activity in broth and biofilm cultures. PloS one 7, e40350 (2012).

3 Kabara, J. J. Structure-function relationships of surfactants as antimicrobial agents. J Soc Cosmet Chem 29, 733-741 (1978).

4 Kabara, J. J. Antimicrobial agents derived from fatty acids. Journal of the American Oil Chemists' Society 61, 397-403 (1984).

5 Kabara, J. J., Swieczkowski, D. M., Conley, A. J. \& Truant, J. P. Fatty acids and derivatives as antimicrobial agents. Antimicrobial agents and chemotherapy 2, 2328 (1972).

6 Yoon, B. K., Jackman, J. A., Kim, M. C. \& Cho, N.-J. Spectrum of membrane morphological responses to antibacterial fatty acids and related surfactants. Langmuir 31, 10223-10232 (2015).

7 Conley, A. J. \& Kabara, J. J. Antimicrobial action of esters of polyhydric alcohols. Antimicrobial agents and chemotherapy 4, 501-506 (1973).

8 Mitri, K., Shegokar, R., Gohla, S., Anselmi, C. \& Müller, R. H. Lipid nanocarriers for dermal delivery of lutein: Preparation, characterization, stability and performance. International Journal of Pharmaceutics 414, 267-275, doi:https://doi.org/10.1016/j.ijpharm.2011.05.008 (2011).

9 Rodríguez, G. et al. Bicosomes: Bicelles in Dilute Systems. Biophysical Journal 99, 480-488, doi:https://doi.org/10.1016/j.bpj.2010.03.072 (2010).

10 van Hoogevest, P. \& Fahr, A. in Nanocosmetics: From Ideas to Products (eds Jean Cornier, Cornelia M. Keck, \& Marcel Van de Voorde) 95-140 (Springer International Publishing, 2019).

11 Rodríguez, G. et al. Characterization of new DOPC/DHPC platform for dermal applications. European Biophysics Journal 42, 333-345 (2013). 
12 Stathakis, V., Kilkenny, M. \& Marks, R. Descriptive epidemiology of acne vulgaris in the community. Australasian journal of dermatology 38, 115-123 (1997).

13 Bickers, D. R. et al. The burden of skin diseases: 2004: a joint project of the American Academy of Dermatology Association and the Society for Investigative Dermatology. Journal of the American Academy of Dermatology 55, 490-500 (2006).

14 Bhate, K. \& Williams, H. Epidemiology of acne vulgaris. British Journal of Dermatology 168, 474-485 (2013).

15 Nutten, S. Atopic dermatitis: global epidemiology and risk factors. Annals of nutrition and metabolism 66, 8-16 (2015).

16 Eichenfield, L. F. et al. Guidelines of care for the management of atopic dermatitis: section 1. Diagnosis and assessment of atopic dermatitis. Journal of the American Academy of Dermatology 70, 338-351, doi:10.1016/j.jaad.2013.10.010 (2014).

17 Center, Q. I., Consult, D. C. \& Apps, A. Skin conditions by the numbers.

18 Rodríguez, G. et al. A unique bicellar nanosystem combining two effects on stratum corneum lipids. Molecular pharmaceutics 9, 482-491 (2012).

19 López, O. et al. Reconstitution of liposomes inside the intercellular lipid domain of the stratum corneum. Langmuir 18, $7002-7008$ (2002).

20 Hallegot, P., MINONDO, A. \& Fiat, F. Cryo-techniques applied to stratum corneum with description of a new sample holder for cryo-scanning electron microscopy of freeze-fractured samples. Journal of microscopy 196, 35-39 (1999).

21 Rodríguez, G. et al. Bicelles: new lipid nanosystems for dermatological applications. Journal of biomedical nanotechnology 11, 282-290 (2015).

22 Coderch, L. et al. The effect of liposomes on skin barrier structure. Skin Pharmacology and Physiology 12, 235-246 (1999).

23 Lima, C. A., Goulart, V. P., Côrrea, L., Pereira, T. M. \& Zezell, D. M. ATR-FTIR spectroscopy for the assessment of biochemical changes in skin due to cutaneous squamous cell carcinoma. International journal of molecular sciences 16, 66216630, doi:10.3390/ijms16046621 (2015).

24 Cevc, G. Lipid vesicles and other colloids as drug carriers on the skin. Advanced drug delivery reviews 56, 675-711 (2004). 
25 Cócera, M. et al. Characterisation of skin states by non-crystalline diffraction. Soft Matter 7, 8605-8611 (2011).

26 Peppas, N. A., Bures, P., Leobandung, W. \& Ichikawa, H. Hydrogels in pharmaceutical formulations. European Journal of Pharmaceutics and Biopharmaceutics 50, 27-46, doi:https://doi.org/10.1016/S0939-6411(00)00090-4 (2000).

27 Ruzin, A. \& Novick, R. P. Equivalence of lauric acid and glycerol monolaurate as inhibitors of signal transduction in Staphylococcus aureus. Journal of Bacteriology 182, 2668-2671 (2000).

28 Schlievert, P., Deringer, J. R., Kim, M. H., Projan, S. J. \& Novick, R. Effect of glycerol monolaurate on bacterial growth and toxin production. Antimicrobial agents and chemotherapy 36, 626-631 (1992).

29 McNamara, P. J. et al. Surfactants, aromatic and isoprenoid compounds, and fatty acid biosynthesis inhibitors suppress Staphylococcus aureus production of toxic shock syndrome toxin 1. Antimicrobial agents and chemotherapy 53, 1898-1906 (2009).

30 Lin, Y.-C. et al. Glycerol monolaurate and dodecylglycerol effects on Staphylococcus aureus and toxic shock syndrome toxin-1 in vitro and in vivo. PloS one 4, e7499 (2009).

31 Shah, P. P., Desai, P. R., Patel, A. R. \& Singh, M. S. Skin permeating nanogel for the cutaneous co-delivery of two anti-inflammatory drugs. Biomaterials 33, 16071617 (2012).

32 Nohynek, G. J., Antignac, E., Re, T. \& Toutain, H. Safety assessment of personal care products/cosmetics and their ingredients. Toxicology and Applied Pharmacology 243, 239-259, doi:https://doi.org/10.1016/j.taap.2009.12.001 (2010).

33 Fluhr, J. W., Feingold, K. R. \& Elias, P. M. Transepidermal water loss reflects permeability barrier status: validation in human and rodent in vivo and ex vivo models. Experimental dermatology 15, 483-492 (2006). 
34 Bazin, R. \& Fanchon, C. Equivalence of face and volar forearm for the testing of moisturizing and firming effect of cosmetics in hydration and biomechanical studies. International journal of cosmetic science 28, 453-461 (2006).

35 Edwards, C. \& Marks, R. Evaluation of biomechanical properties of human skin. Clinics in dermatology 13, 375-380 (1995).

36 Luthra, A. Complications of Mesotherapy. Complications in Cosmetic Dermatology: Crafting Cures, 143 (2016). 